Fall 2000

\title{
2000 Miracle Yearbook
}

Cedarville College

Follow this and additional works at: https://digitalcommons.cedarville.edu/yearbooks

Part of the Higher Education Commons, Organizational Communication Commons, and the Public Relations and Advertising Commons

\section{Recommended Citation}

Cedarville College, "2000 Miracle Yearbook" (2000). Yearbooks. 29.

https://digitalcommons.cedarville.edu/yearbooks/29

This Book is brought to you for free and open access by DigitalCommons@Cedarville, a service of the Centennial Library. It has been accepted for inclusion in Yearbooks by an authorized administrator of DigitalCommons@Cedarville. For more information, please contact digitalcommons@cedarville.edu. 

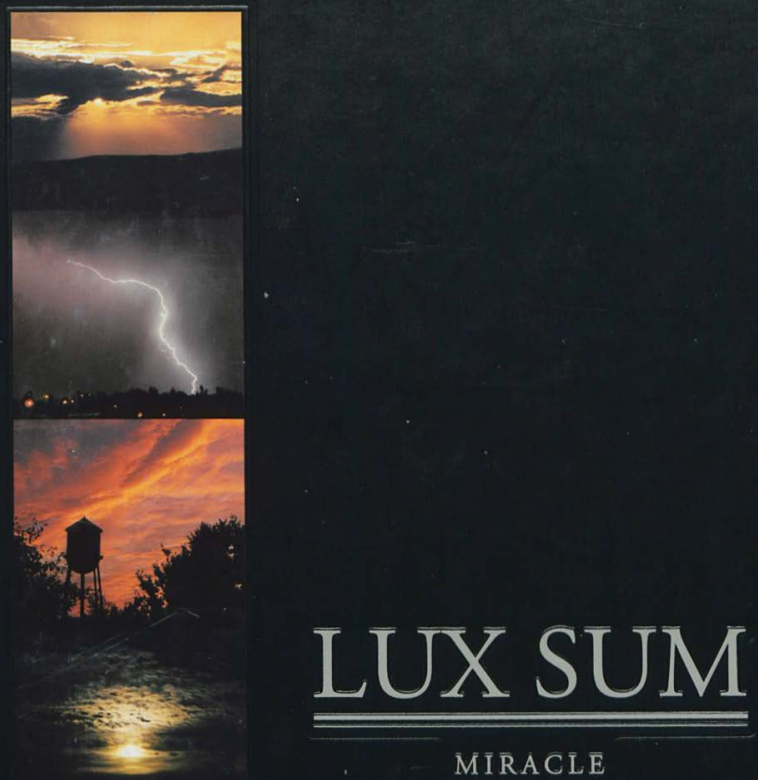

Mİ $\bar{R} A \bar{C} \bar{L} \bar{E}$ 2000 
Please Return To:

EXTERNAL

RELATIONS 
I AM THE LIGHT 



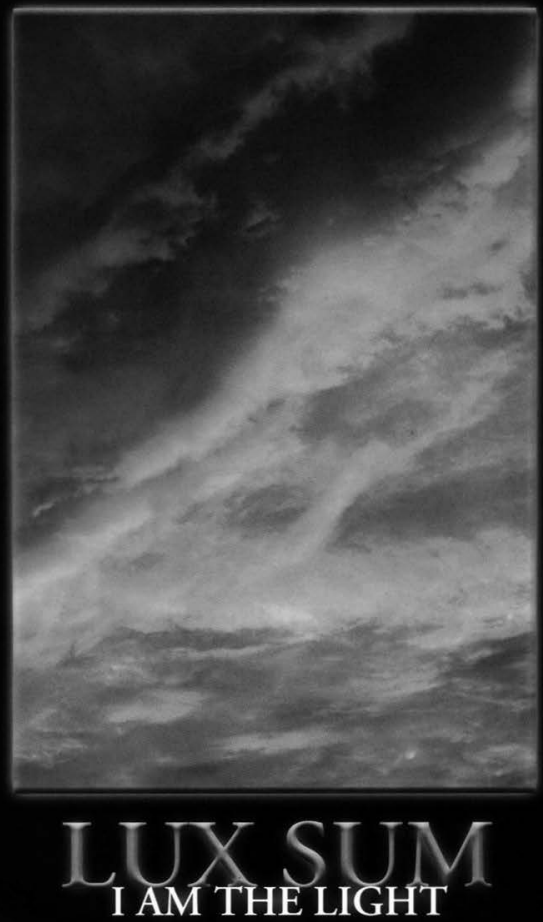

Miracle 2000

volume forty-seven 


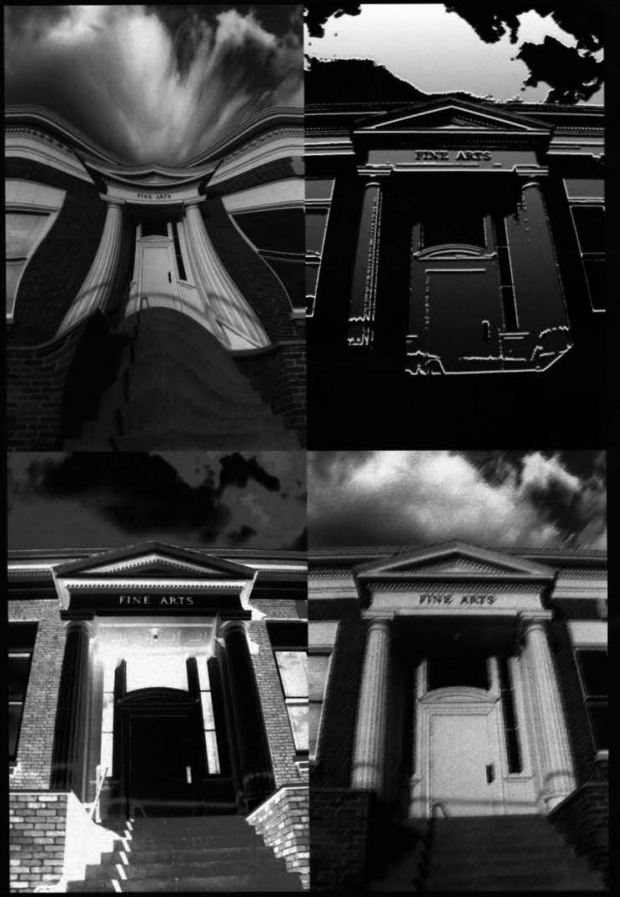




\section{ACTIVITIES 12}

Chapel 42

ACADEMICS 58

SENIORS 80

UNDERCLASSMEN 130

ARTS 174

MiNISTRIES 186

OrganizATIONS 222

Athietics 246 

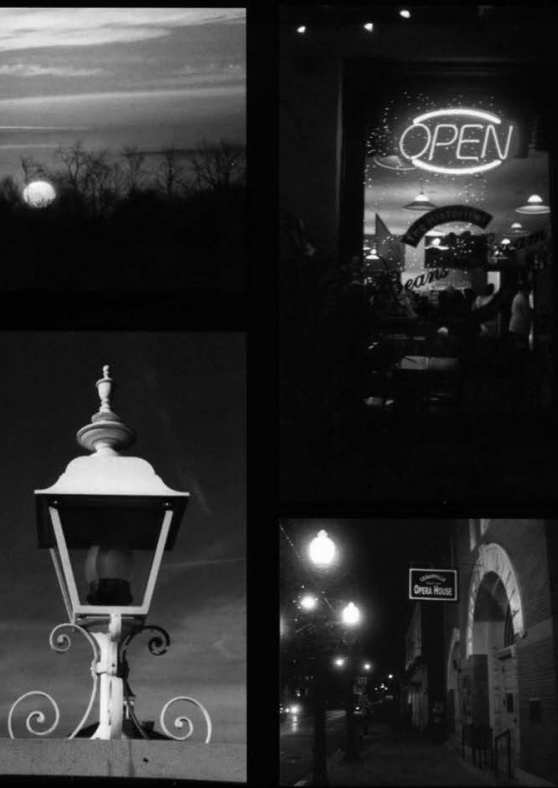

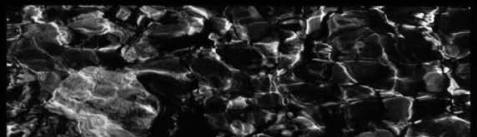

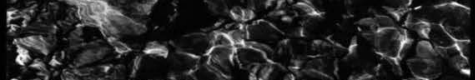

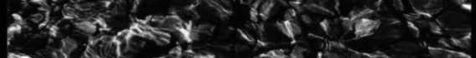

I am the Light of the world.

Whoever follows me will never

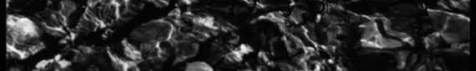

$x^{2}$ है $\rightarrow$ aर की 

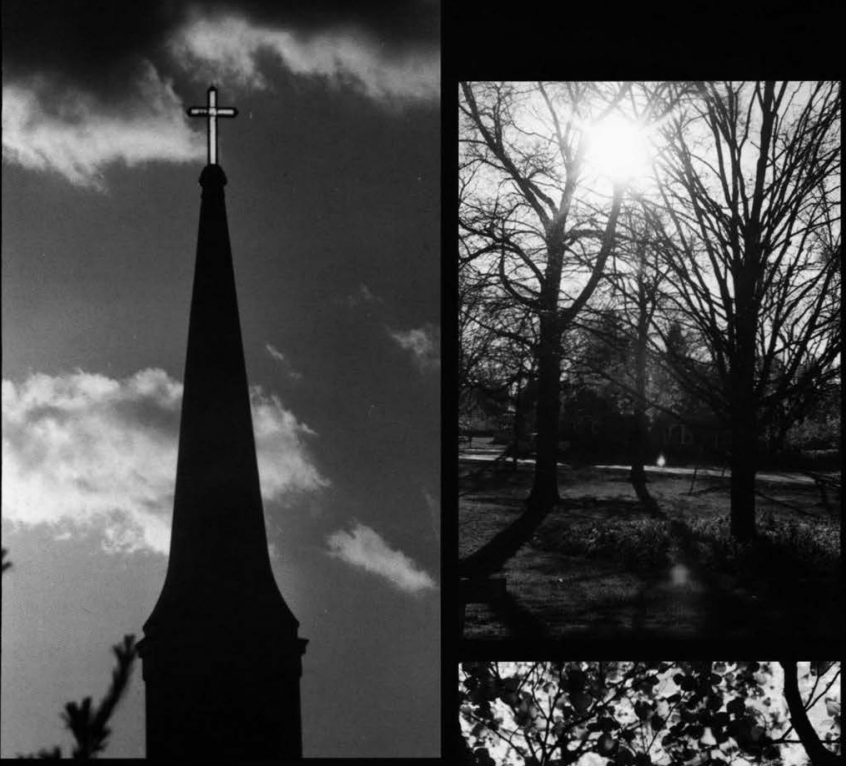

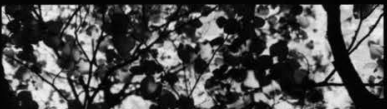

walk in darkness,

but will have the Light of Life.

John $8: 12$ 

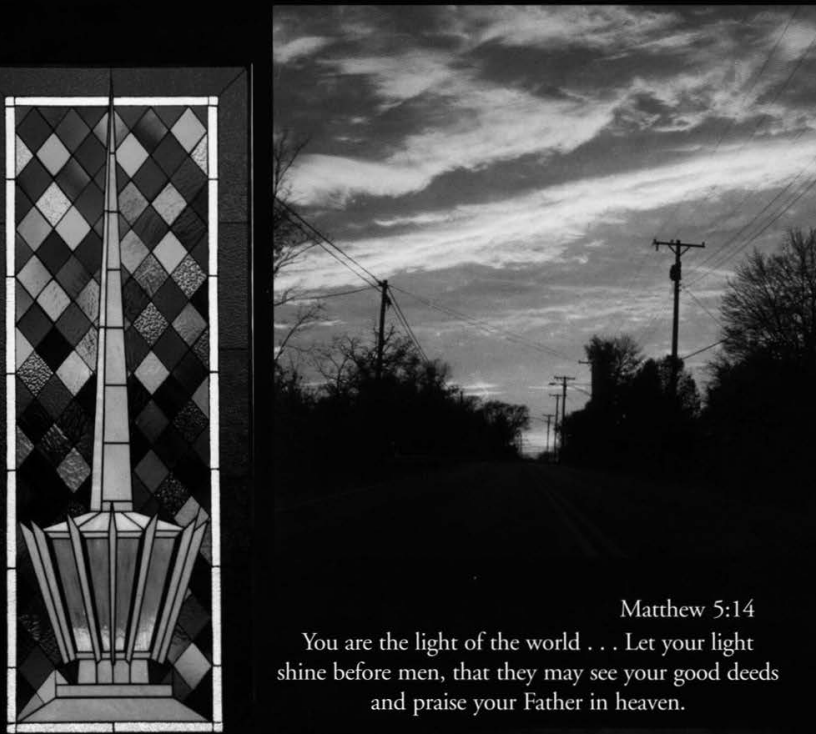

Matthew 5:14

You are the light of the world ... Let your light shine before men, that they may see your good deeds and praise your Father in heaven.
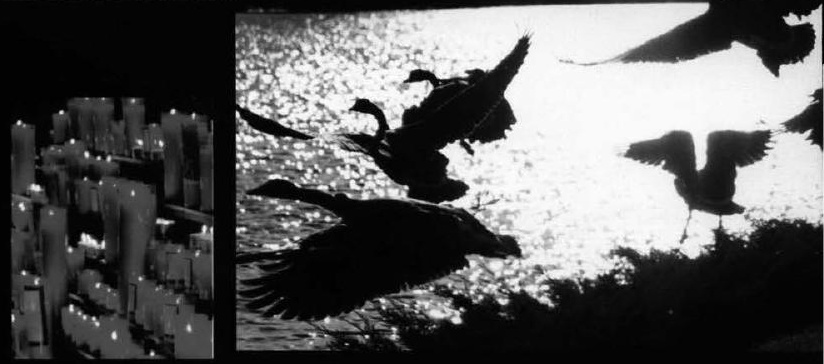


\section{Siving in the $\Gamma$ igft}

"Let your light shine before men, that they may see your good deeds and praise your Father in heaven." Matthew 5:16

$W$ faw him every day in chapel and twice a week for Fellowship. Yet, probably few students knew the countless hours that Pastor Robert Rohm had devoted to serving the student body here at Cedarville. Beyond his visible role as Campus Pastor and Vice President of Christian Ministries, those whoknew Pastor Rohm attested that he spent countless hours on his knees and at his desk to fulfill God's call on his life-to work with college students.

Pastor Rohm first came to Cedarville in 1964 when he left Audubon, N] to becomea college freshman. Graduating in 1968 with a business major and a triple minor in Bible, psychology, and Greek, he and his bride then headed off to Dallas, Texas where he entered the custom clothing business. After approximately six years in that business, Pastor Rohm realized that God wantedhim to serve elsewhere. In obedience, the Rohm family headed to Bethel Baptist Church, in Michigan, where Pastor Rohm accepted the position of associ. ate pastor. For the next 12 years, Pastor and Mrs. Rohm developed a passion for working with both college-age and single adults.

F 1986 the Rohm family returned to Cedarville, and a short nine years Lafter their return, Dr. Dixon ap. pointed Pastor Rohm to become the Vice President of Christian Ministries. This new position thrilled the Rohms because, like his pastoral experience, it

involved an abundance of contact with college students. As Lynn, his wife of 33 years explained, "[Bob] just had a love for God and a love for the Word. He also enjoyed that his job centered around students $\sim$ he enjoyed helping students in this important time in their lives."

his love for God and college
students was evidenced in Pas-
tor Rohm's daily life. Senior Ann Weeks remembered the time that Pastor Rohm broke his leg shortly before the school year began. "He was still so full of spunk," she recalled. "He never missed a day of chapel, and he always put others' prayer requests before his own."

Many others appreciated the fact that Pastor Rohm went out of his way in chapel, at Chuck's, or walking along the sidewalks, to talk to students. In addition, Pastor Rohm was a beloved actor of the SGA videos, often putting aside his image to become everything from a GAP model to a buff, tough Campus Security Officer.

Pastor Rohm's simple acts of kindness, selflessness, and humility were what truly set him apart from scores of other pastors and Christian leaders, for although his challenges from the Bible were always powerfully delivered, it was his behind-the-scenes laboring and caring that made Pastor Rohm truly unique.

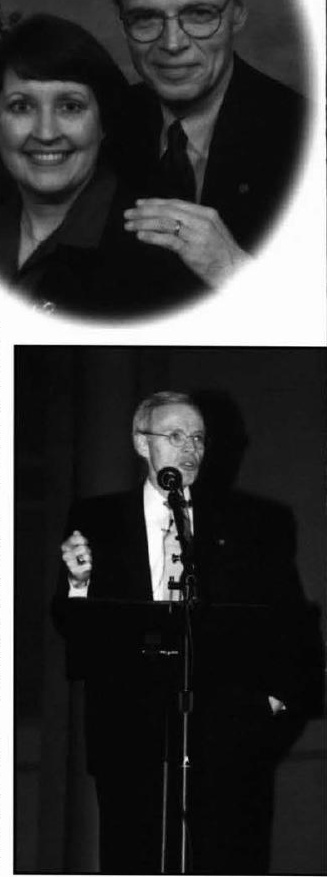

Pastor Rohm presents powerful messages that challenge the student body to live out their faith daily. 


\section{Siving with}

"Jesus said, 'I am the resurrection and the life. He who believes in me will live, even though he dies; and whoever lives and believes in me will never die." Joln 11:25-26

\section{Matt Hadler}

Mart Hadler was at Cedaryille for just six months, but in that time he became a close friend and great teammate to a number of track athletes and others at the college. Hadler transferred from the University of Wisconsin-Milwaukee at the beginning of winter quarter 1999 . He came to Cedarville looking for a place to be encouraged spiritually and to do what he enjoyed most with a group of Christianathletes. Hadler waspassionate about running. He was committed to his teammates and dedicated to the training that is necessary to succeed.

Many people were attracted to Hadler because he enjoyed almost everyone, and he wouldn't let the little things in life bother him. He had a wild streak that ran deep despite his quiet demeanor, and people admired him for his knack for having fun and ability to enjoy life.

Eddie Nehus was a good friend and often one of Hadler's training parmers on the cross country and track teams. ${ }^{4} \mathrm{He}$ was one of the [greatest] guys to bearound. He was one of the most dedicated nunners l've ever known. Every day in practice he was the one to push every interval.. He was always in a good mood, and nobody ever had any problems with him. It seems like there are not enough good things to say about him," Nehus said.

Hadler's contributions to the teams were many even though he was only here a short time. Coach Elvin King said, "Matt set the tone for the workouts, which was kind of amazing because he'd been here less than a year. He was a leader. He was fun-loving...He never knew a stranger... He looked for ways to encourage people."

Hadler loved his first six months at Cedarville and was looking forward to getting back after the summer. He had arranged to live with three other seniors on the track team for the next year. In mid-September, Hadler and two others had moved into that apartment.

On Sunday September 19t Hadler was on his way to meet his family for a Packers/Lions football game in Detriot. Along the way he had an accident with his motorcycle which claimed his life.

Many friends and teammates were able to attend Hadler's funeral in Wisconsin. Hadler's mother Susan said, "I just want the students to know how much Cedarville meant to Matt. He was so excited about going back and seeing his friends and the kids on the cross country team. We appreciate the coaches and kids who came to Matt's funeral and memorial service. As much as we miss Matt -and we miss him very much -we appreciate all the school did for him."

Teammate Drew Nelson said, "I probably knew more of who he was just from hearing people talk about him. I didn't know him very long. He worked like an animal; every race he ran, he ran as hard as he possibly could. He did the same thing in every area of his life. He told me once during cross country camp about how he really enjoyed last year and walking around campus and never hearing the Lord's name taken in vain. He really loved God, and you could see that."

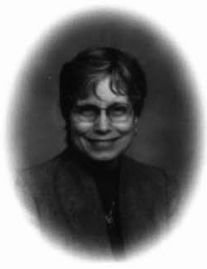

\section{Dr. Sharon Eimers}

Sharon Eimers developed a strong family bond of love as a child in the small town of Ripley, New York that she would carry with her for the rest of her life. Sharon Eimers was the first born of seven children. When she was thirteen months old, her late father wrote in a letter to her, "My dearest darling daughter, l ask thisprayer that God, with your own help, will bring you through life's trialsand tribulations and may you grow up to be wonderful, beauriful, and lovely like your mama."

It was this loving devotion that Eimers shared with her family and her students. Sharon and Lee were married on August 24, 1962, in First Baptist Church of Ripley, New York. Together, they reared three girls: Tami, Kim, and Renee, who received all the love and devotion their parents could offer. When the Eimers' daughters got married, they graciously extended their love and care toward their sons-in-law.

After a long battle, Sharon Eimers succumbed to bone cancer during the spring quarter of 2000 . She taught in the education department at CedarvilleCollege, instilling in her students her desire to impact and educate.

"She was an amazing woman and a great testimony for us all. One thing I'Il always remember her teaching us is that we are never too busy to take the time to pray. I know that she believed in the value, necessity, and power of prayer, and showed us that by the way she lived," said one of her students, Nadine Tornsa.

Mary Zuleger said, "When 1 think of Dr. Eimers, 1 


\section{Helen Mae Fischer Drullinger}

The woman known to hundreds of students, we did our best to give the kids as normal a home faculty, and staff as Dr. Drullinger's "sweetie" joined the Lord she loved on September 22, 1999.

Helen Fischer graduated from one of the first secondary Christian schools in the United States and received a diploma in Bible from Multnomah School of the Bible. From the beginning of her Christian training, she felt called to be the wife of a pastor or a fulltime Christian worker. This calling was fulfilled when she met Dave Drullinger in San Francisco, where he was serving in the Navy. They married just six months after they met.

Mrs. Drullinser worked as secretary to a pastor while Dr. Drullinger finished serving in the Navy and completed his education. They had two children. "Obviously, there were sacrifices that she made in not being a stay-at-home mom, yet

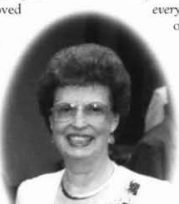

i. possible," said Dr. Drullinger. "We did everything together...I often wonder if other couples ever even approach the level of oneness that weenjoyed so much."

She continued her service as a pastor's wife when Dr. Drullinger began pastoring the Calvary Baptist Church of Pleasanton, California. "Helen had the spiritual gift of helps and was content to be a quiet and gracious pastor's wife," said Dr.

Drullinger. "She was observed by many to be the 'perfect' pastor's wife, so devoted was she to her hushand and the ministry to which they hadbeencalled." After the Drullingers came to Cedarville, she worked in the college post office as a postal clerk. Even in illness, she remained faithful, "She was a godly woman, a gracious pastor's wife, devoted mother and grandmother, loving sister, sister-in-law and friend," said Dr. Drullinger.
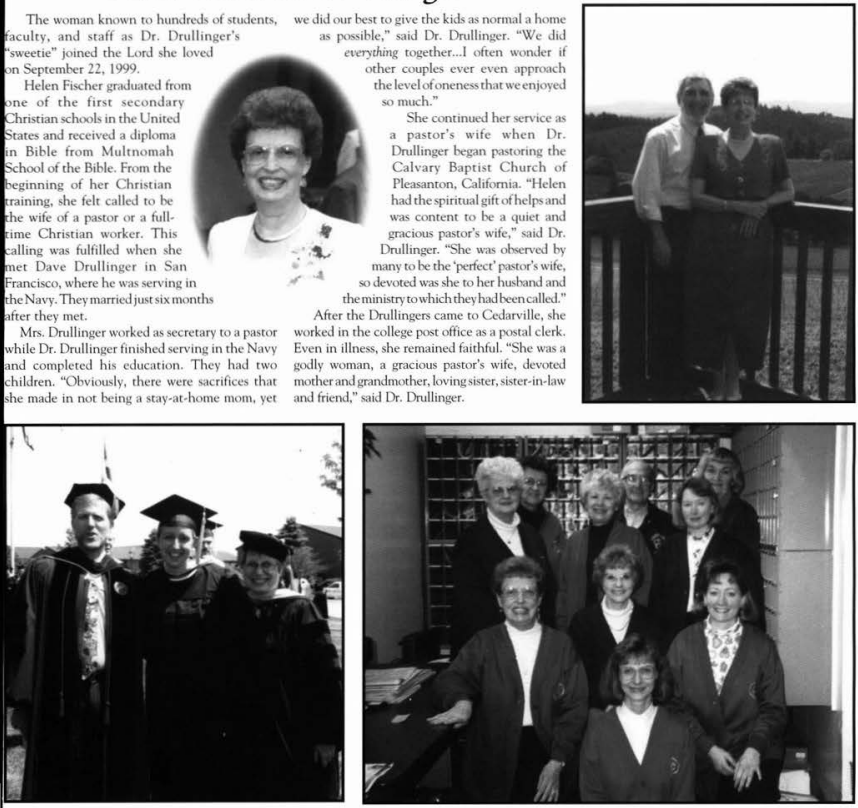

think of her enthusiasm and her love for teaching and life. 1 learned so many things from her in the things she didn't say. Her life always pointed to God. One thing I can remember her telling us is to make sure we ask the people in our lives what are the things that they feel are important for us to do for them. She had the heart of a servant."

"We were sitting in a public school hallway," said Susa Hayden "when she told me the cancer had returned, and explained that she was ready for her homegoing. Right there, with kids walking by, I cried with her. Her hope in the face of problems inspired me to stop doubting and believe that God's purpose and plan is good."

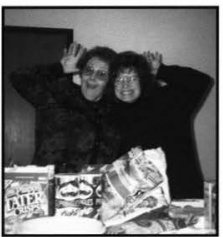

The 2000 Miracle lovingly remembers thiese members of our college family who have gone to be with our Lond. We extend our prayers and comfort to their friends and families, remembering that our hope is in the promise of eternal life through Jesus Christ our Lord. 
God is light; in Him there is no darkness at all . . 


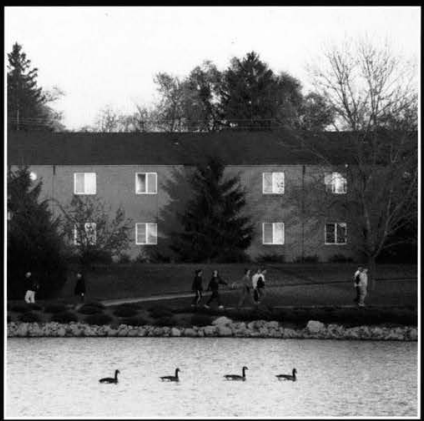

ACtivities 


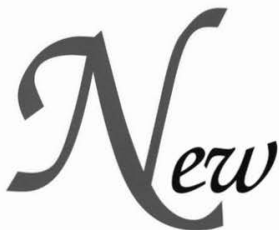

The Cedarville College campus was a buzz of excitement as over $\operatorname{SG} \mathcal{A}$

encouraged small

groups to "Cean on"

\section{each other} to get past the newness of college life. one hundred volunteers welcomed in the class of 2003 and many transfer students for the annual "Getting Started weekend." After receiving warm smiles, friendly helps, a balloon, and a picture with the Yellow Jacket, their first experience as a Cedarville College student had begun.

On Friday evening, the first night on campus, new students were the honorary guests at the "Early Arrival Party," featuring everything from human foosball to wacky prizes from the campus radio station, U99.5. Saturday afternoon brought the new students their first college courses, including Cedarnet 101 and pointers in how to "call home" successfully and effectively.

To help with the adjustments and initial shock of being in college, each new student was placed in a small group. The fun-loving SGA encouraged small groups to "lean on" each other to get past the newness of college life:

Sunday morning the Cedarville College Fellowship held a special service for students and their families. Later that day, after giving hugs and shedding tears, it was time to say good-bye to parents and family members. As the event-filled weekend came to a close with the taking of the freshmen class picture, the many volunteers lined the sidewalks, cheering the freshmen on, as if to officially welcome them into the college family.

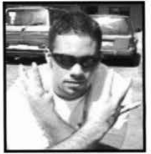

Micfiael Tharp embodies the excitement of Getting Started.

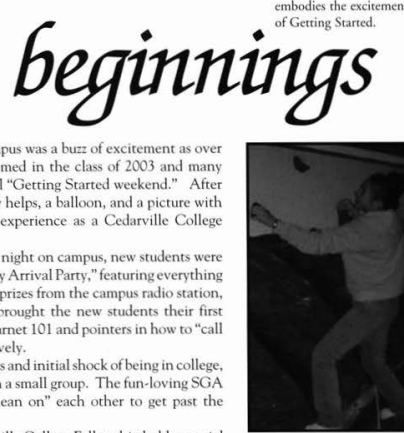

Marty activitics, such as this rock-climbing wall, await new students at the early arrival party.

Volunteers help with the tedium of unpacking vehicles after many long journeys.

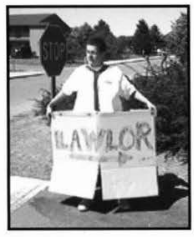

$\mathcal{Z}_{\text {he men of Lawlor }}$ rake it upon themselves to make new students feel right at home.

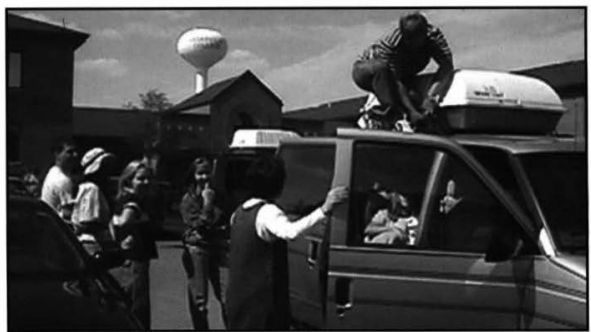

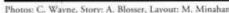




\section{- Peger is}

Q⿱宀

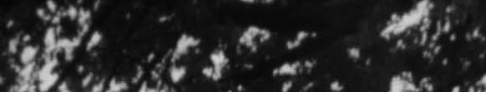

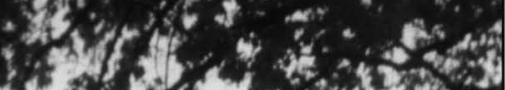

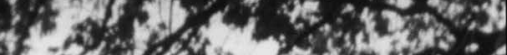

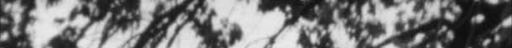

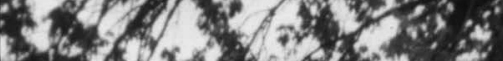
I. $x, x^{2}+x^{2}$

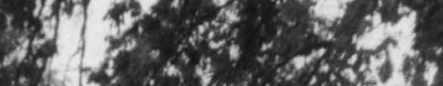

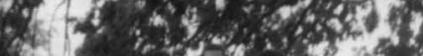

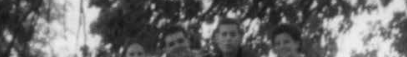

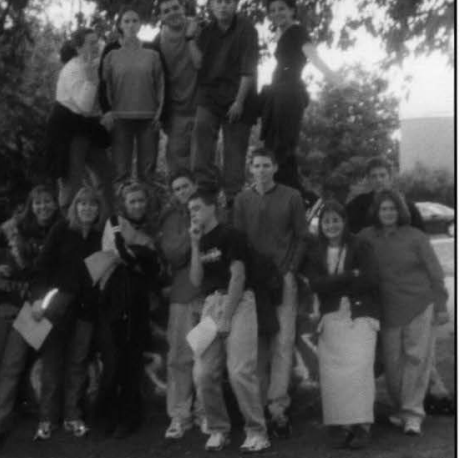

Small group tradition: the tour of the 'Ville scavenger hunt, with a stop at the famous Rock.

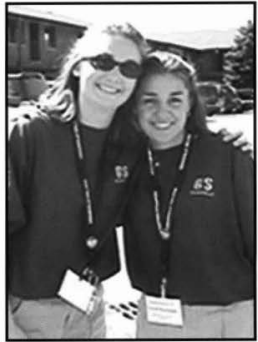

$A_{\text {manda }}$ Schmuck and Crystal Stamanich stop for a pose despite the huscle and bustle of their many Getting Started responsibilities.

"The shadiest thing I did as an RA during Getting Started..."

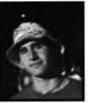

"...was walk around at the early arrival party incognito as a new student."

Trief Whston
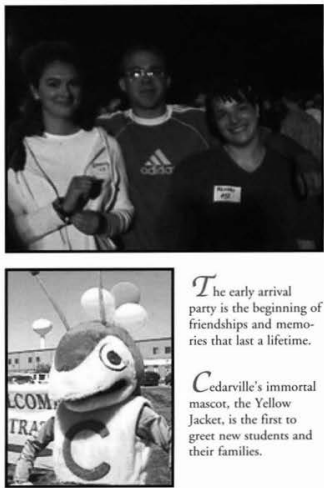

$\mathcal{T}_{\text {he early arrival }}$ party is the beginning of friendships and memories that last a lifetime.

Cedarville's immortal mascot, the Yellow Jacket, is the first to greet new students and their families. 
$\mathcal{Z}_{\text {he Brock RA's pose on the car they }}$ demolished at their block party.

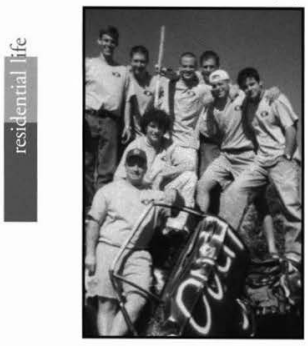

"The thing I like most
about dorm life..."

"...there is never a dull moment when you live with a bunch of guys."

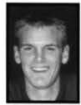

Robert Marsiall

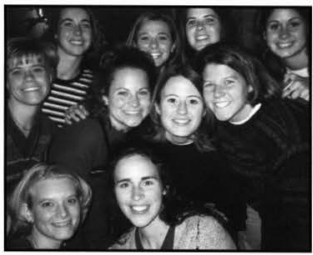

The Harriman girls celebrate the end of another week of classes.

Jennifer Ingersol and a few of her friends spend time together at the transfer picnic.
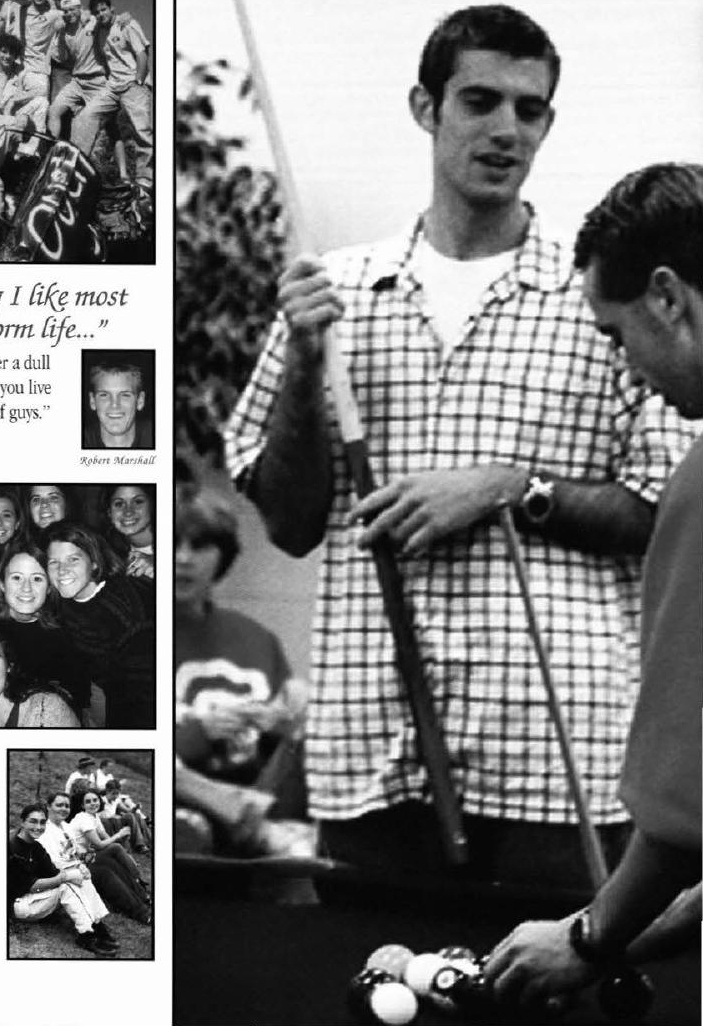
Chris Freeman

relaxes after classes with

a game of pool.

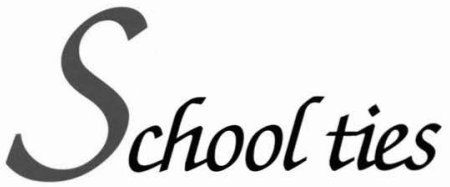

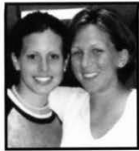

Annette Ruba and Kelly Kokeny have some fun after a long day of soccer camp.
Friendsfips at Cedarville provide students

with study buddies, weekend

\section{companions,} and spiritual supporters.
Papers, exams, quizzes, homework and projects forced students to the library and study lounges. Despite the work, the college years can be the best time of one's life. Surrounded by peers, college students always found time for the most important aspect of their college career, developing social skills! Yes, spending hours at Chucks, weekends at a friend's house, visiting John Bryan for some time with God's creation, having a cup a Joe at Beans and Cream, painting the Rock, and dating all kept students away from studies during their time at the 'Ville.

Cedarville furnished students with a unique blend of activities and a kind of residential life unlike anywhere else. Pick-a-dates allowed unmet lovers to become acquainted with each other. Unit and hall activities supplied camaraderie among mates. Ma and Pa's gave students a quick place to eat when Chuck's just was not enough. Cedarlake gave the campus its aromatic attraction. The Rock furnished students with a bulletin board to post messages from birthdays to marriage proposals. The cities of Dayton, Columbus, and Cincinnati provided refuge to those who just wanted to get away.

Whatever students did for fun, the most important part of residential life to most people was the spiritual support. Prayer groups, Christian ministries, and Bible studies all gave Cedarville students a spiritual boost. Life at Cedarville was not only a time for studying and fun, but most essentially it was time for spiritual growth.

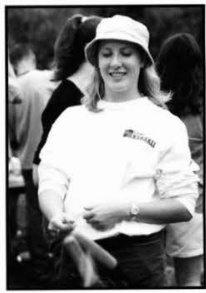

A. new transfer student enjoys grilling her hotdogs at the transfer picnic.

Kelly Hadings and Sharon McMurray bond on their blow up chair.
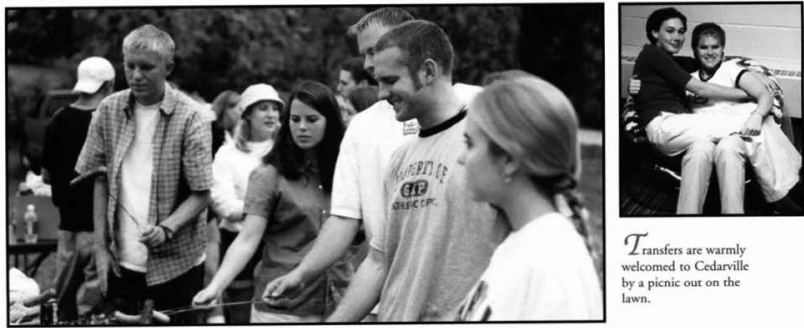

$\mathcal{L}_{\text {ransfers are warmly }}$ welcomed to Cedarville by a picnic out on the lawn. 


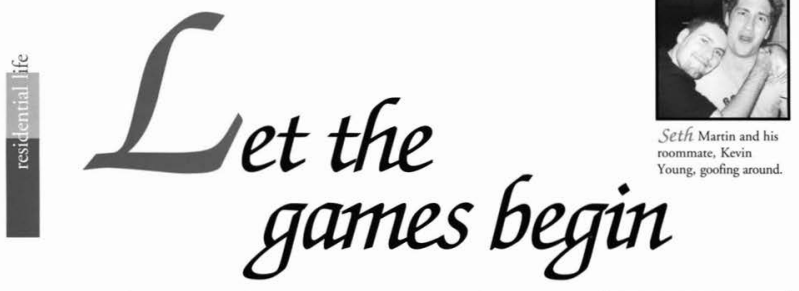

Dorm Mlars It started early in the morning. As students walked to class, they DOTML WaTS were looking over their shoulders. What were they looking at? In w conversed about assassins. Could there be anything wrong? Could it happen here at Cedarville? No! lt's just Dorm Wars!

time!

\section{Dorms}

competed in

several

\section{activites}

floping to

win first

place.

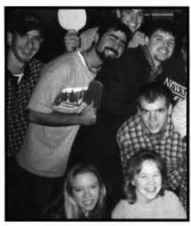

$S_{\text {tudents find rime }}$ away from studies to play ping pong in the Miter center.
Dorm Wars was an SGA sponsored activity to create camaraderie within each dorm and find out which were the "best" men and women's dorms. There were several games played out on the track and hammer-field. Each representative from a dorm that won their match in an activity received points for their dorm.

Asassins was a game that involved strategy. Each participant received a card with another participant's name on it. Their mission: to seek them out and "assassinate" them. But players needed to be aware; the enemy was out there!

Plank Wrestling was a true test of one's strength and agility. Opponents faced each other on an elevated plank five feet off the ground. Participants used only one hand in an attempt to make their challenger lose their balance and fall onto the mats.

Target was a test of accuracy. Participants launched water balloons at a giant red dot. The closer to the target, the more points received.

The competition in Dorm Wars was great. It was thrilling to see Dorm Spirit. The women's dorm winner was Printy Hall, and the men's was McChesney Hall.

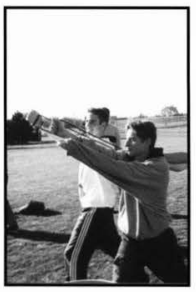

Gabe Lopez and Brock Weston shoot water balloons as they participate in Target.

Chris Headings and Jim Hulbert fight it our in Plank Wrestling!

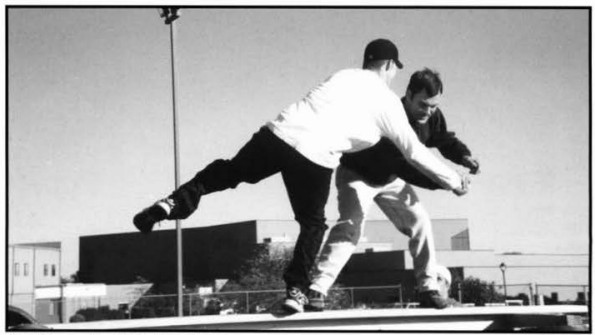

Photes: K. Leverson, Storye R. Mitchell. Layout:S. Airtama 
$\mathcal{K}$ yra Gray and some of the girls in her hall spend the afternoon together.

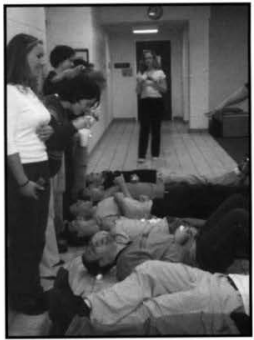

$I_{\text {is }}$ always hard to predict what will take place during a dorm activity?

\section{"I would recommend living in a dorm because..."}

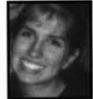

“...living in a dorm has brought me so close to many different people."

Xisthy Yokinown
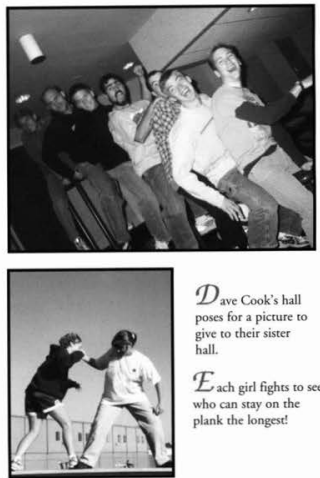

Dave Cook's hall poses for a picture to give to their sister hall.

$\mathcal{E}_{\text {ach girl fights to see }}$ who can stay on the plank the longest? 
With enthusiasm, fans show their support of

Jason Marshall.

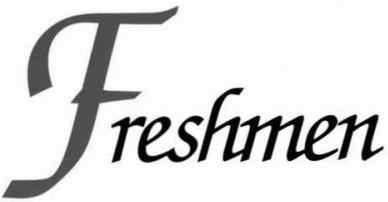

What does it take to make a

\section{canoe}

that will

\section{survive}

Cedar

Lake?
Braving Cedar Lake, freshmen engineers competed in the Cardboard Canoe Race on October 15 , 1999. 50 feet by 44 inches of Mead cardboard, 100 meters of packaging tape, and creativity were all Nathan Foote, Phil Holdren, Evan Miller, and Dana Shaver proved this notion to be possible by successfully navigating across the lake in two minutes and fifteen seconds. Their team won this annual mechanical engincering competition, better known as the "Canoe Race."

The race this year was all about teamwork. Despite having only three weeks to prepare for the contest, the freshmen engineers spent many hours discussing, designing, and building their boats. The winning team, along with upperclassman advisor Chris Recktenwald, concentrated on the strength of their boat, sealing their victory with reliability rather than looks.

Although designed to test the spirit of the new engineers, the canoe race had become an institution for all disciplines at Cedarville. Though two that was provided to make it across Cedar Lake.

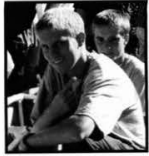

Ieff Ganong prepares for the upcoming challenge.

teams from other majors or organizations - U99.5 and a group of business students - participated merely for the fun of overcoming the lake and sealing their names in the annals of Cedarville history, the engineers competed for a new calculator and a good grade. The students gained points for each cone that they passed as their boats wound snakelike across the water.

Despite the low number of students who actually wanted to brave the waters of Cedar Lake, throngs of students from every class and major gathered to watch the beginning engineers.

A select group of senior engineering majors had such fond memories of the race that they could not resist participating again. Although some would argue that attempting to sink the freshmen boats by launching water balloon missiles is not participation, the activity brought a thrill to the crowd and motivation to the freshmen.

Unfortunately some freshmen engineering boats did not complete the voyage across the lake with the ease of the winning team, proof that Cedar Lake teaches many lessons.

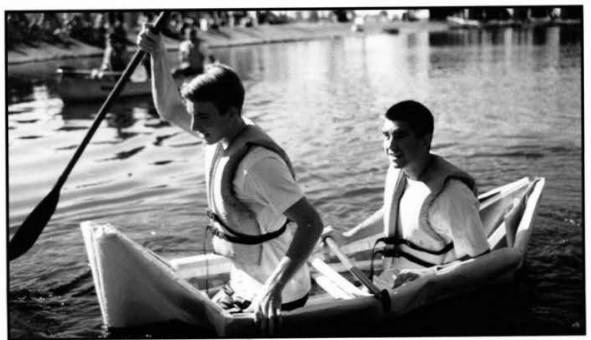

Photos: K, Leversen, Story Aaron Warrieer, Lyour K. Tyoo

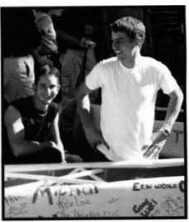

$\mathcal{J}_{\text {eremy Patton and Chad }}$ Keiser pose with thier vessel. $G_{\text {reg Reilly and Steven }}$ Bruchart struggle to remian afloar. 


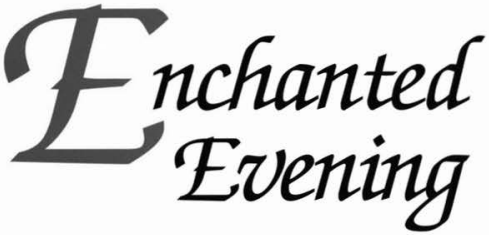

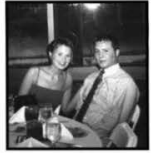

Lindsey Borland and Craig Stevenson enjoy dinner in the Alumni Hall.

\section{Dining in} the DMC

\section{and singing in the}

sanctuary makes this

banquet a night to remember.
Dubbed "An Enchanted Evening," the 1999 Homecoming Banquet featured Chicken Wellington and an after-dinner concert with 1996 American Songwriter's No. 1 Christian Artist of the Year, Scott Krippayne, and fatherson duo Aaron Jeoffrey. For the first time that students remembered, the banquet was not held in the Athletic Center. Instead, students ate at tables set up in the Dixon Ministry Center as the AC floor received another coat of varnish. To some, this alone was enough to make Homecoming 1999 a memorable event. "I really liked having it in the DMC," said senior Jeremy Cunningham. "I thought that was a major step up from the AC." Senior Sarah High, who helped plan the event, said that it had been hard not knowing where the banquet would be. "We didn't know officially where we would be until four days before Homecoming," she said. "The main thing I'll remember is that we were almost homeless for Homecoming."

For most of the seniors, spending the evening with friends was the most memorable. "I'll remem. ber it because it was the first time all of us got dressed up and went out together," said senior Patty Thompson. For many seniors, it produced mixed emotions, as they realized they were finishing their college years. "Our entire table was full of seniors, thinking that it was our last formal together," said Sarah High. Senior Grady Peeler noticed the humorous side of the evening more. "It was pretty memorable when Joel came out to announce Aaron Jeoffrey and accidentally started to announce $\mathrm{Mr}$. and Mrs. Aaron Jeoffrey," he said.

Other students thought the concert was the high point of the evening. Amy Stowers said, "It was great. The concert was very worshipful." At the concert's end, the audience joined the singers in singing "Shout to the Lord." Senior Andy Hedges found it hard to pinpoint the highlight of the evening. "The great food, inspiring music, and, of course, the opportunity to spend time with a special person in my life all came together to make homecoming an evening to remember."

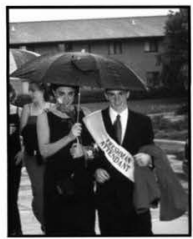

$\mathcal{D}_{\text {ustin }}$

Walker

escorts

Leah Gombis and dates prepare to the DMC. for a night of fun!

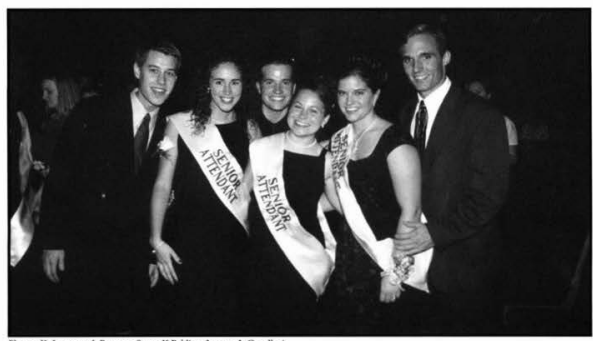

Photos: K. Leverion, L.Burman, Storys K.Belding, Layout: J. Cantilani 


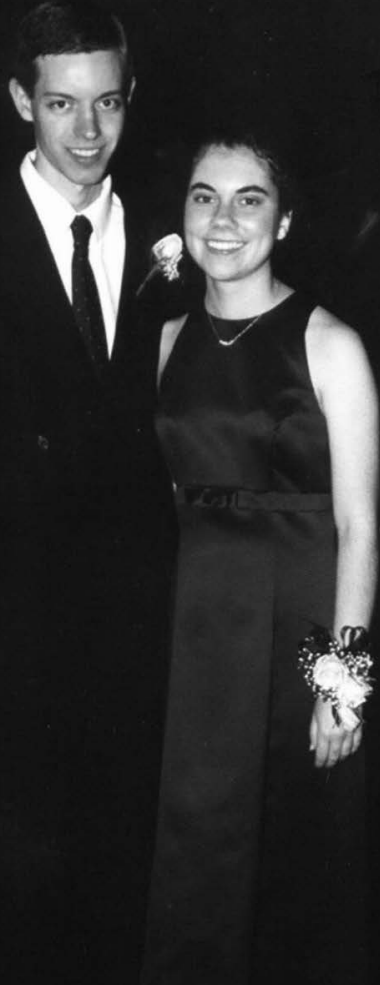

Adam Rorex and Jeanette Shrier share a moment together.

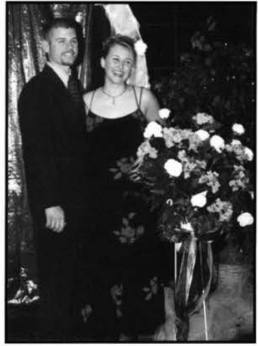

"My most memorable moment was..."

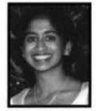

"...having my husband by my side, holding my hand."

Yaya Yofianna:
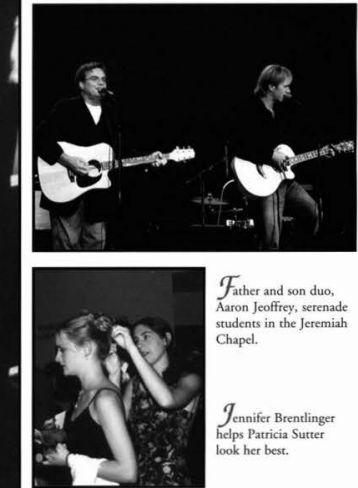

Father and son duo Aaron Jcoffrey, serenade students in the Jeremiah Chapel.

\section{$\mathcal{J}_{\text {ennifer Brentlinger }}$ helps Patricia Surter look her best.}




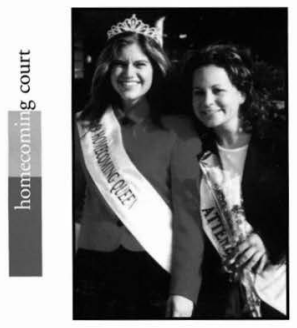

NiKk $i$ Thomas and Kelsey Perkins share good times at the homecoming parade.
$\mathcal{E} d$ Spencer crowns Nikki Thomas Homecoming Queen for 1999.

Proverbs 31:30

"Charm is deceitful and beauty is vain, but a woman who fears the Lord, she sfiall be praised."
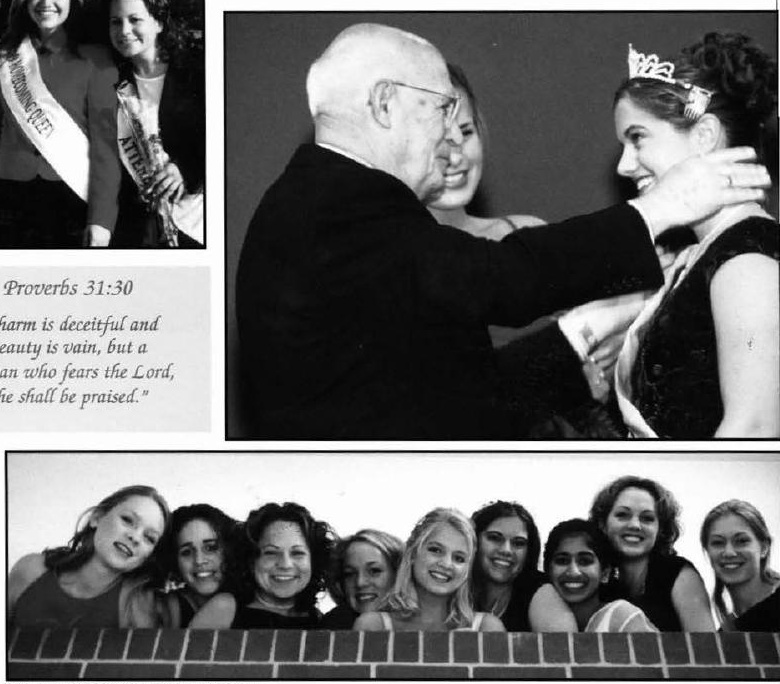

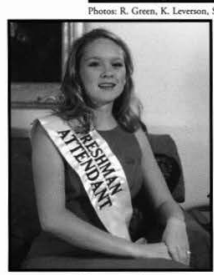

Sfuae Elam

Freshman Attendant Undeclared Hebron, IN

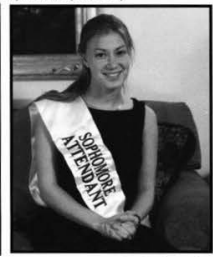

Megan Mofirland Sophomore Attendant Music Education Kalamazoo, MI

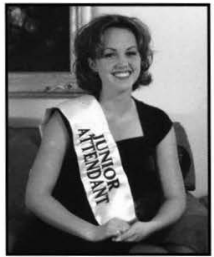

Krista Spain

Junior Attendant Nursing East Liberty, $\mathrm{OH}$

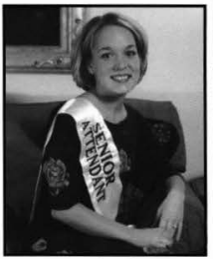

Saraf Miniet Senior Attendant Communication Arts Williamson, NY 

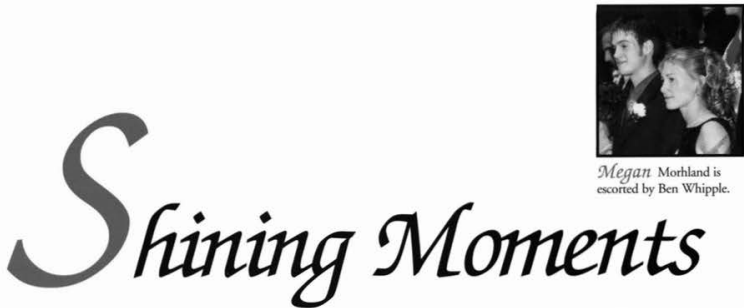

The

Nikki Thomas, the 1999 Homecoming Queen, had loved Cedarville ever since arriving here from her hometown near Flint, MI. Nikki involved herself in many activities like Concert Chorale, CAB, ADO, crowning

of nine ladies

\section{allows}

them to

share

God's

work in

their lives. and MENC. After graduation, Thomas, a music education major,

planned on obtaining her master's degree in music therapy at graduate school. She was escorted at the banquet by her fiance.

1999 scnior attendents included Sara Minier, Jaya Yohannan, Kelsey Perkins, Shanna Lenhart, and Geneva Roberts. Minier, a Communication Arts major, was "honored to be on homecoming court." Sara was escorted by her dad, who was a Cedarville alumnus. Yohannan, a nursing major with a cross-cultural minor, said she enjoyed giving her testimony abour what God had done in her life. She was escorted by her husband, Jacob. Perkins, an organizational communications major participated in the Dayton Detention Home Ministry, Delta Phi Sigma, and Society for Human Resource Management. Lenhart, a nursing major, planned on doing pediatric or maternity nursing. Shanna was organizations director of SGA and in Delta Phi. Shanna was escorted by her fiancé, Scott Leht. Roberts, an elementary education major, experienced her fourth year on the court and was escorted by her brother, Micah Roberts.

The junior attendent was nursing major Krisra Spain, who said, "It was such an honor to be on homecoming court and a lot of fun." The sophomore attendent was Meghan Morhland, a music education major, involved in band and orchestra. The freshman attendent was Shae Elam who was glad to be on the homecoming court saying, "I got to represent my Lond and my class."

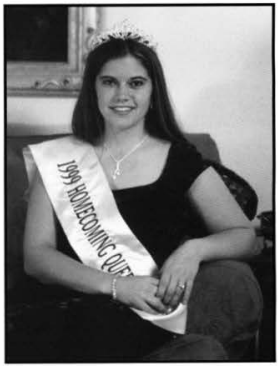

Nikfi Thomas Homecoming Queen

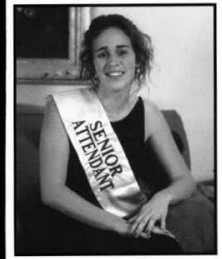

Geneva Roberts

Senior Artendant Elementary Education Milton, VT

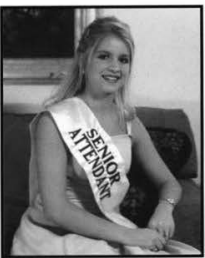

Sfianna Lenfiart

Senior Attendant

Nursing

Grand Blanc, MI

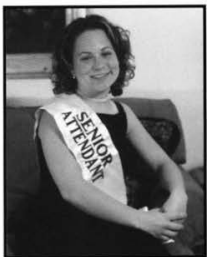

Kelsey Perkins

Senior Artendant

Organizational Comm.

Aurora, $\mathrm{CO}$

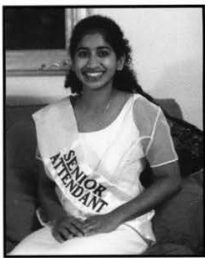

Jaya Yohannan

Senior Attendant

Nursing

Westwood, NJ 

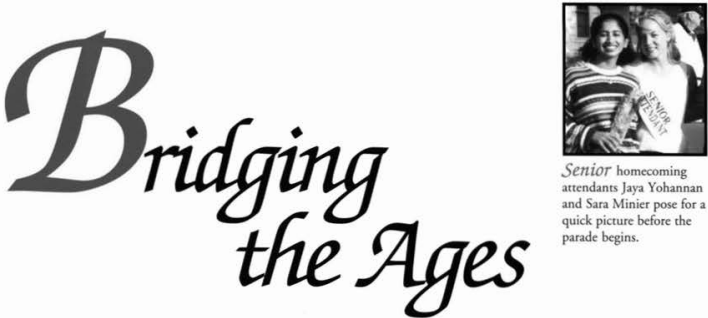

Senior homecoming attendants Jaya Yohannan and Sara Minier pose for a quick picture before the parade begins.

The annual fornecoming parade allows

\section{students}

to display their works of creativity and fumor.
Even the weather cooperated for the last Homecoming Parade of the 20 th century, with blue skies and Indian-summer temperatures. Leaves littered the streets and a fall breeze drifted past the crowd as the homecoming parade marched through downtown. The parade's theme was "Bridging the Gap of Time" and emphasized the constants that hold true over the years. Alumni returning for homecoming weekend reunited on the sidewalks of Main Street. Lining the street, students and alumni watched the floats head through Cedarville.

Participants in the annual march down Main Street included all four classes and numerous organizations, as well as Cedarville township organizations such as the fire department. Custodial Services entered their first float, featuring a giant toilet. The freshman class entered the winning float, which showed the changes between the stone-age Flintstones and the space-age Jetsons. Fred Flintstone's feet powered his car as he yelled Yabba-Dabba-Do. At the other end of the float, Elroy and Jane displayed their spacey outfits.

The freshman win ended the three-year winning streak of the class of 2000 , giving the freshmen a jolt of self-confidence. As freshman secretary Becca Brummel said, "Our float was really good, and it won!" Townspeople, students, faculty, staff, and alumni truly enjoyed the fall weather and the last homecoming parade of the century: "Bridging the Gap of Time."

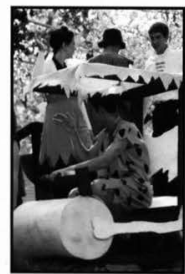

Fred Flintstone drives his car at the front of the freshman float.

Mu Kappa dresses up to represent the different countrics of the world.

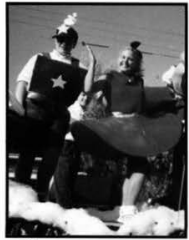

$\mathcal{L}_{\text {he back of the freshman float }}$ features the Jetson cartoon characters.

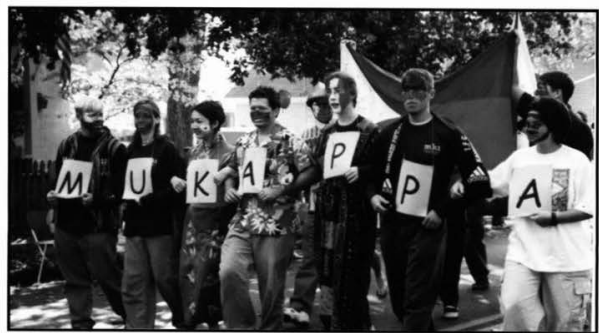

Photos: C Vipram, Sroory R. Moll, Layour: R. Camer 
celebrates a "nerdville"

Christmas with Rachel

Beach, Denaye Hilty,

Charlie Brown, Jennifer

Sreely, Katie Shank,

and Melissa Bielo.
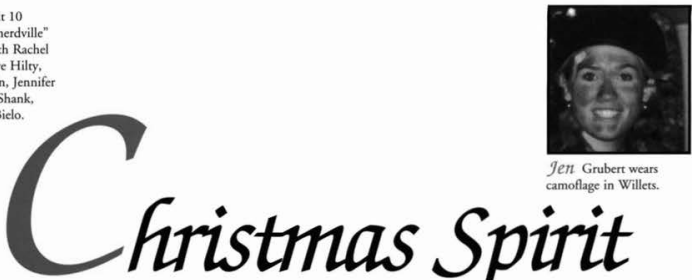

Ien. Grubert wears camoflage in Willets.

Christmas decorations

and

original

Open House

thernes

bring

Christmas

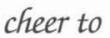

carnpus.
As children growing up, one of the fondest memories was decorating for Christmas. Children would pester their parents until they reluctantly pulled out the Christmas tree and lights. At Cedarville College, there was an annual tradition that kept that memory fresh and alive for many students. Many of the women spent hours preparing their dorm for Open House. From door to door the students showcased a managerie of themes. While strolling through Faith Hall, students found themes such as Christmas in a Nursing Home or in glittery Heaven. One could see Christmas at the Circus or even get flushed down the toilet to see Christmas in the Sewer. Adam and Eve showed their visitors what Christmas was like in the Garden of Eden. One unit gave sleigh rides in their lounge. Many dorms offered candies and cookies since food was a popular part of Open House. In Willetts, one could experience the beauty of Christmas in New York City including the beautiful fountain and ice skating at Rockefeller Center. Many halls gave students a fright as they took them through boot camp, while McKinney offered Christmas in the ski lodge. One of the most popular halls was Johnson's Christmas in the White House. While walking past the blue room, one could meet President Clinton or even brush shoulders with Dr. and Mrs. Dixon as they toured Open House. The ladies at Cedarville College did an excellent job keeping the spirit of Christmas decorating alive, and Open House was a definite success.

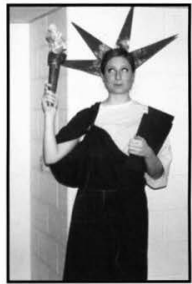

Elizabetft Witley represents the Statue of Liberty.

Chrissy Wheatley poses as Popeye the Sailor in Johnson's open house.

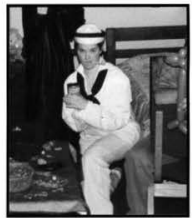

$\mathcal{E}_{\text {rin Vallowe and unit } 18}$ of Printy enjoy a wild sleigh ride. 

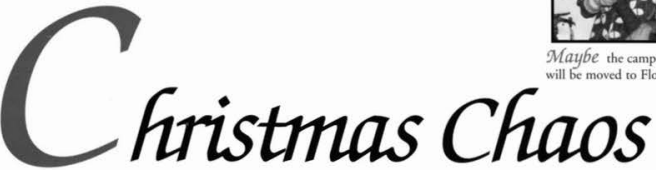

Open frouse

is an eyeopening

experience

for many

Cedarville

\section{women.}

The truth

finally

comes out.

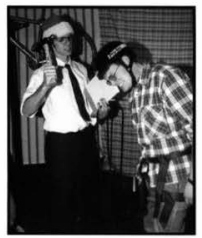

$\mathcal{K}$ evin Leverson works as quality control for Santa in his free time.
The memories of making snow angels in the yard and licking icicles hanging from the roof all remind us of our childhood at Christmastime. However, no snowball fight with the neighborhood children could have ever topped the WWF thumb wrestling found in one Lawlor unit. No sledding down the hill with eight kids piled on a one-man toboggan ever brought the same excitement that many students felt when they experienced Christmas under Cedar Lake for the first time. From the very modern "Y2K Christmas" to the more traditional "Santa's Workshop" and everything in between, the men succeeded in demonstrating a wide range of imagination and creativity. However much we progressed when we stepped onto this campus, and however hard we tried to leave home behind, many aspects of the men's residence halls this holiday season still reminisced of childhood days. The "Soda Can Christmas," complete with aluminum mantle and fireplace, brought back warm memories of reclining before a blazing fire in the living room. Although we've learned by now that eating snow is harmful and that it is much too dangerous to pile eight people on a one-man sled, we were able, for one evening, to forget all our scholarly knowledge and just enjoy the celebrations of Christmas, past and present.

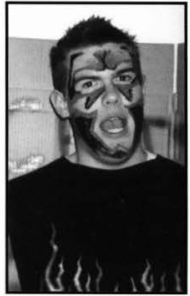

Marshall Lyons sings: "O Christmas Tree, O Christmas Tree!"

Wes Tillett, Dave Buchanan and Dan Fries join the ranks of famous reindeer.

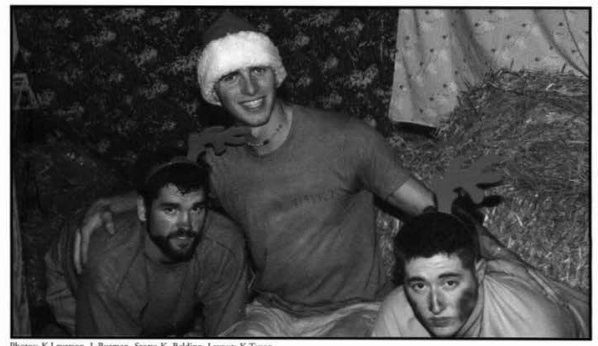




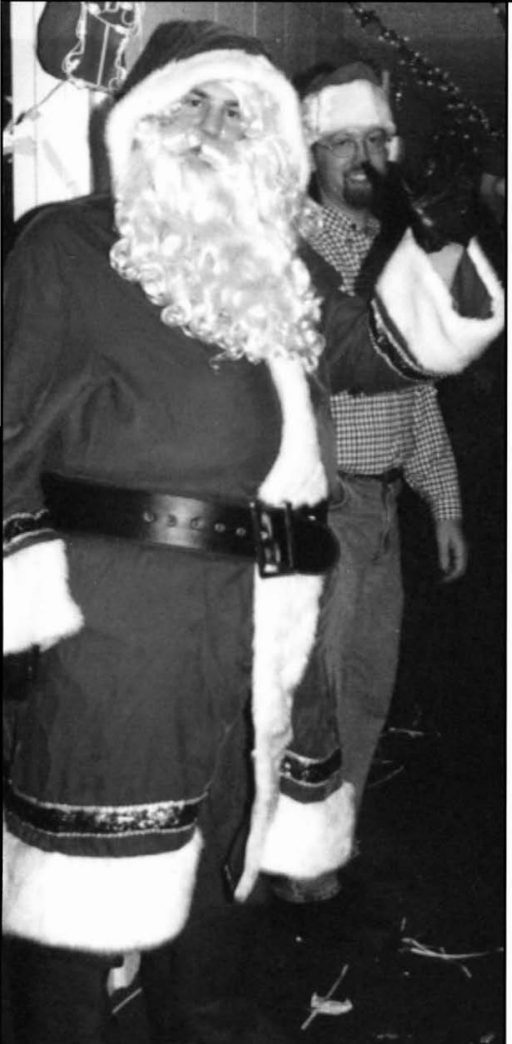

$O_{\text {pen House would not be complete }}$ without a jolly Santa Claus.

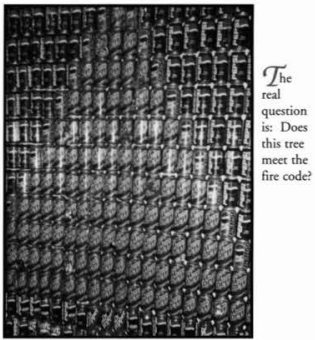

"The thing I dread most about Open House is..."

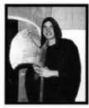

"...that somone might find the dirty socks I have hidden under my bed."

Watt Garret:
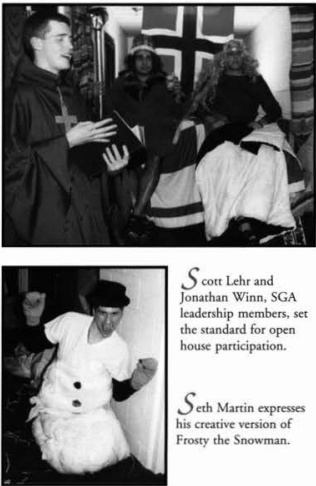

$S$ cott Lehr and Jonathan Winn, SGA leadership members, set the standard for open house participation.

Seth Martin expresses his crearive version of Frosty the Snowman. 


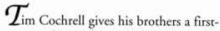
hand look at the U99.5 studio.
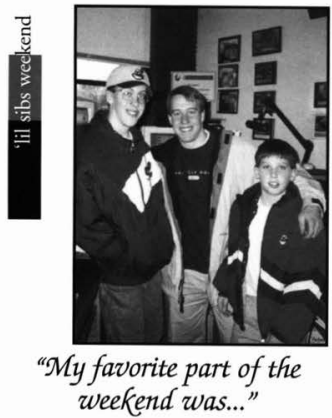

"...going to the Winter Blast with my big brother Ben and running through the obstacle course."

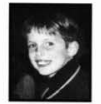

David Gayer
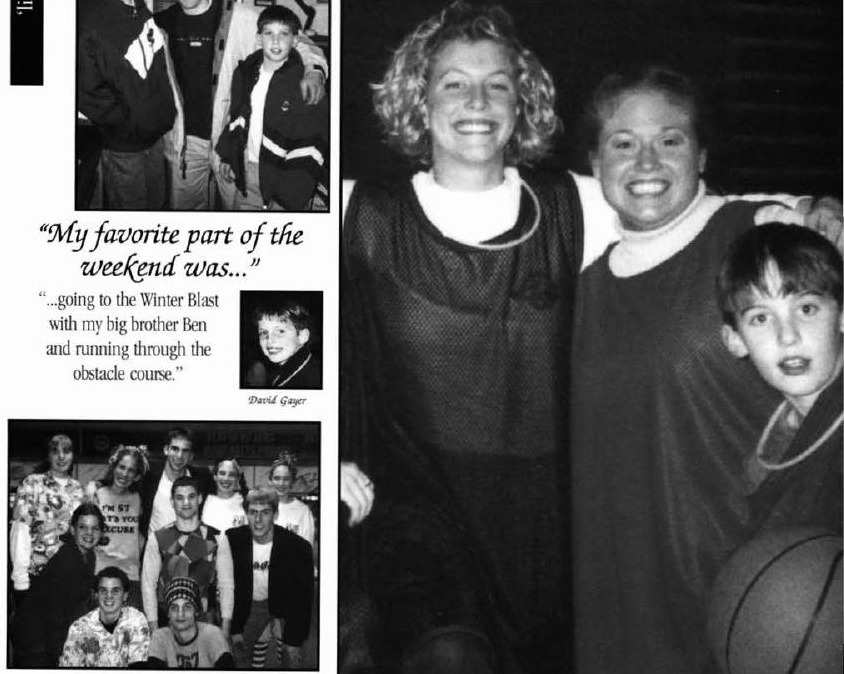

Some people will use any excuse tobe crazy.

$\mathcal{M}$ idnight bowling proves to be a success for friends Christy Heflin and Stephanic Airtama and sister Andrea Aittarma.

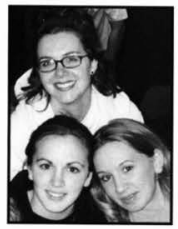


Basketball is a bit morc challenging, and much more humorous when Jill Burz, her brother, and friend Natalie have to wear these shoes.

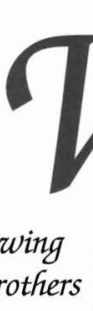
and sisters

is a

highlight for both

students

\section{and siblings.}

Showing

\section{(1)} off brothers

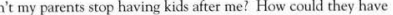

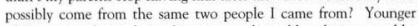
siblings were always doing things to get their older sibs into trouble. However, when we moved away from home, their influence on us was greatly minimized. Our perspective changed. We could better appreciate their diverse personalities, amazing ability to amuse us, and knack for always finding trouble. Whether four or fourteen, six or sixteen, they will always be our "little" brothers and sisters.

In light of this "new appreciation" gained by college students, Cedarville continued the tradition called Li'l Sibs weekend. Students braced themselves as once again their little sibs took over. Actually, Li'l Sibs weekend provided an opportunity for an incredible bonding experience. Students got the chance to have their younger siblings stay in their rooms and follow them around campus for the weekend. In all seriousness, having little sibs on campus brought a lot of enjoyment for Cedarville students. Aside from the fact that it provided diversion from the never-ending studies, it was awesome to see family again, even if they were li'l sibs.

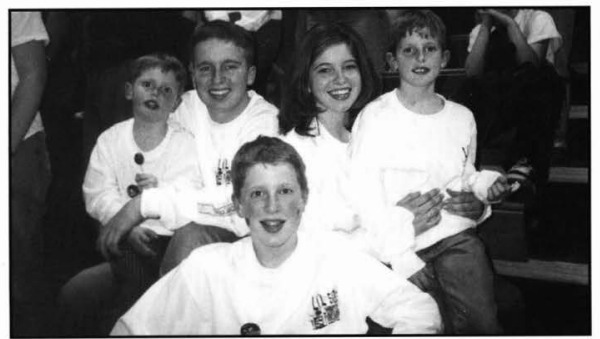

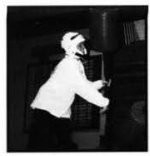

American Gladiators at Cedarville College?

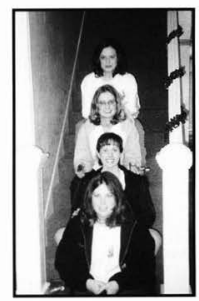

Chrissy Redfearin and her roommate Danielle show her sisters what dorm life is like.

Friends Jill Osmon and Rebecca Gapinski get their picture taken with their sisters on the sleigh.

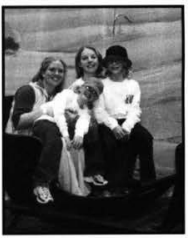

$\mathcal{B}_{\text {en and Emily Gayer attend }}$ the basketball game with younger brothers Stephen, Jeremy, and David. 


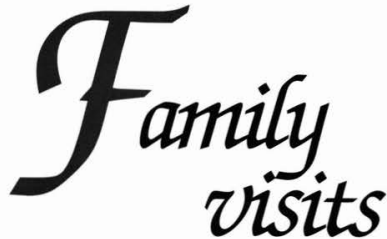

Each spring, Cedarville College holds Parent's Weekend. Parent's weekend began with the Honor's Day Chapel. The DMC was crowded with excited family members, especially those whose children were being honored. Jaya Yohannon, Senior Nursing Major/Cross-Cultural Minor received the Ruby Jeremiah Scholarship which was given to a female married student. She was joined by her parents and in-laws who traveled from New Jersey to see her.

Friday night there was a Pops Concert. The school play was performed both Friday, and Saturday night. On Saturday, there was a Golf Scramble for students and parents and other family members. Saturday afternoon, there was the first tour of the newly opened Student Life Center. Parents witnessed the unfinished structure of the next year's newest and largest build. ing. At night there was a Michael Card concert.

Becca Carl, freshman nursing major, was not expecting to see her parents that weekend. Her parents told her that they were not able to visit because of her father's work. However, her mom contacted her boyfriend and roommate and found a way to get her to stay on campus Thursday night. At 7:15 Friday, her mom pulled up to her boyfriend's house, and her dad joined her the next day. They were able to attend the golf scramble and go to church with her the next morning. Becca said, "Overall, it was an incredible weekend of fellowship together. I'm so thankful to the Lord for such loving and Godly parents and am also so thankful for the sacrifices they made to be able to come up here this past weekend. It was a very special gift."

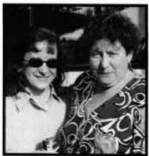

Visits from Mom and Dad are welcome anytime.

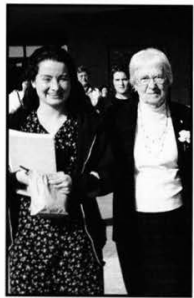

\section{Dad in the}

spring.

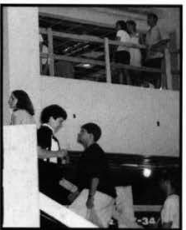

$\mathcal{P}_{\text {arents and students }}$ have the opportunity to tour the Student Life

Center under construction.

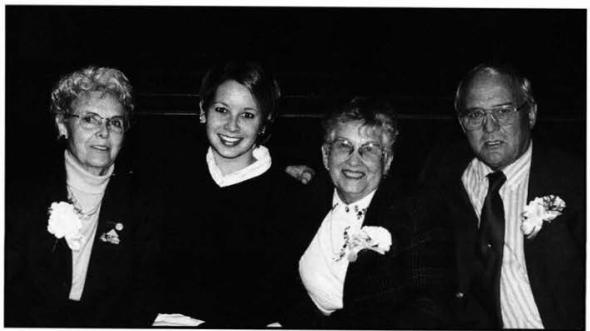

Photos N, Vitall Storg: R. Moll Luyeut: B. Gayer

Saraft Dunn escorts

her grandmother out

of the chapel service.

\section{Grandparents are}

recognized and honored during the chapel hour. 
Micfiael Card performs in the

Jeremiah Chapel on Saturday evening

during Parents Weekend.
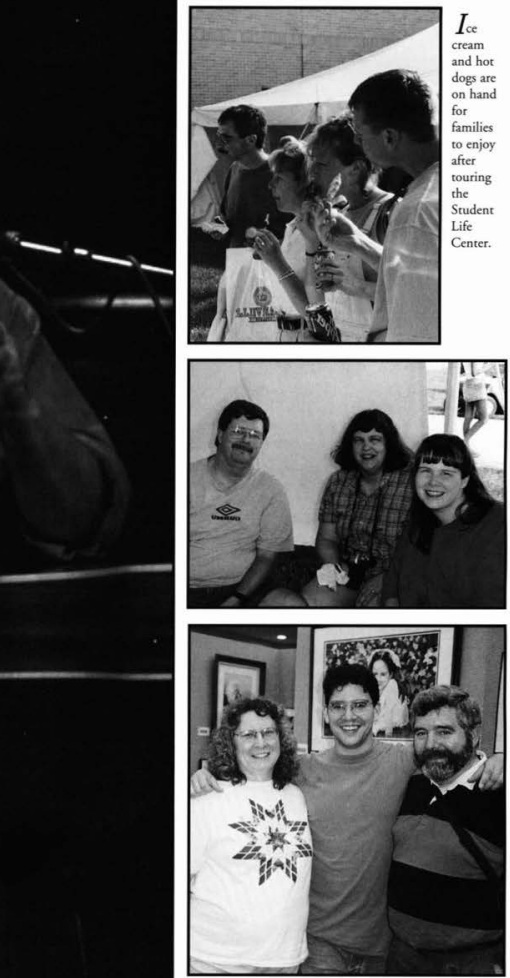

$\mathcal{T}_{\text {he weekend is full of }}$ activites for parents and students to take part in. 


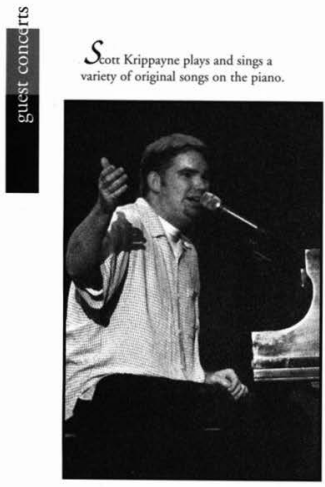

\section{Instrumental groups are also featured on the} big stage.
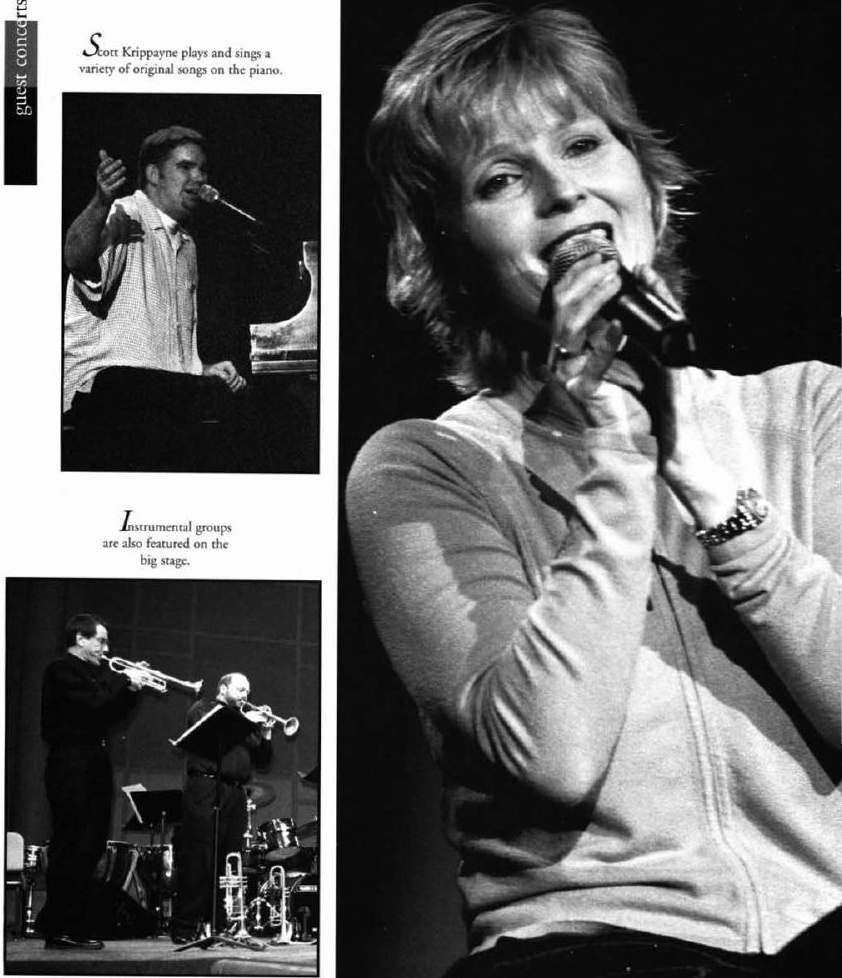
Twila Paris ministers in song to students and alumni alike during homecoming weekend.

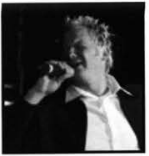

With passion, the lead vocalist of $\mathrm{FHH}$ sings out.
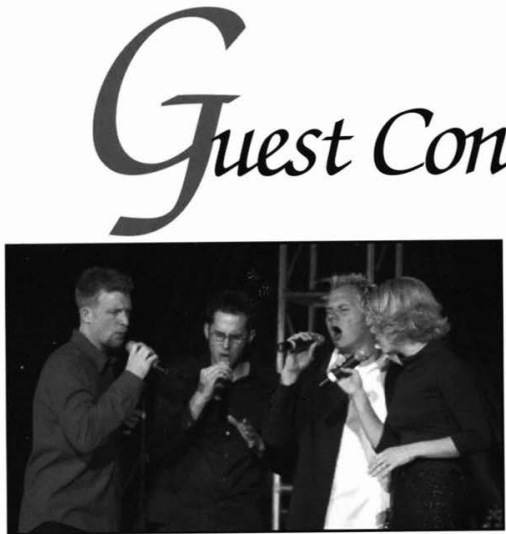

Throughout the acedemic year, various artists visited the college to entertain and uplift students.

Some groups and individuals like $\mathrm{FfH}$ and Twila Paris used their vocal talents to minister to the entire college family.

Carillion Brass and Ira Stein also came, utilizing a variety of instruments to play many different tunes.

Michael Card and Chris Rice came with a few back up artists but primarily played and sang original music. "I liked the fact that Chris Rice sang his own songs and played them for us on the guitar and piano. I thought it was an excellent concert," said sophomore Nicholas Schlappi.

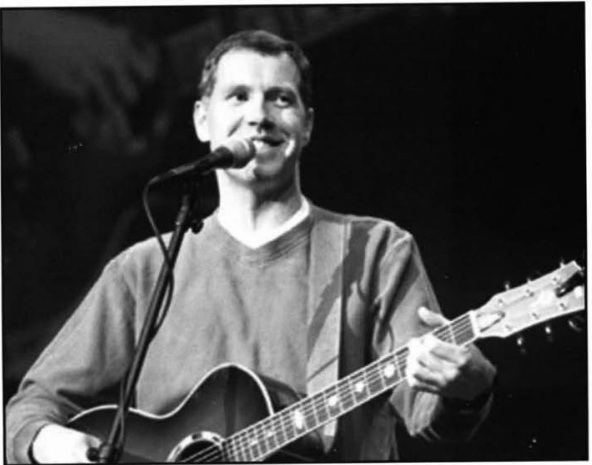

$\mathcal{F} f$ Hused their incredible vocal talents to entertain students for the second straight year at Cedarville(above left).

Chris Rice(left) comes to campus to minister in music and song.

$g_{e f f}$ Beste, who organizes some of the campus concerts, meers Chris Rice before the concert.

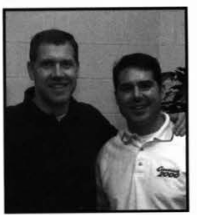




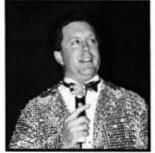

Dr. Coleman hosts the annual Talent Show.

\section{In the}

annual

Alpha Chi

talent show,

\section{students}

provided

a wide

variety of

\section{entertain-}

ment.

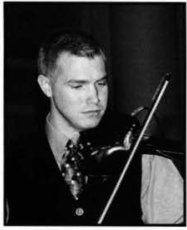

$\mathcal{M}_{\text {iah Thirey enterains }}$ the crowd with his original electric violin solo.
TWIRP was the acronym for The Woman Is Responsible to Pay. This term referred to the initiative of the Cedarville women, who during the designated week, arranged and funded their dates.

The kick-off event for TWIRP week was the annual Talent Show. Created in 1967 by the Alpha Chi organization under the leadership of Dr. Murdoch, the event continued on for thirty-three years, and had become a tradition. Emcees Dr. Coleman and Dr. Clevenger incorporated the TWIRP theme by using their matchmaking skills and giving out gift certificates for local restaurants.

The performances of the evening included a large variety of students and displayed a wide range of talent. Taking first place in the musical category was the group that called themselves Guys from Lawlor. Their piece entitled, "Dear Sister," was written by a member of the group and reflected on the struggle that many girls experience with eating disorders. The musical performances ranged from singing to saxophone and even included an electric violin.

In the category of non-musical talent, the Cedarville Martial Arts Ministry Team won first place. Their demonstration incorporated forms of jousting and kickboxing, with a performance by the Brockstreet Boys. Other non-musical performances consisted of drama, computer animation created by Shave Sevo and Matt Plaatje, and a balancing act performed by John Myers.

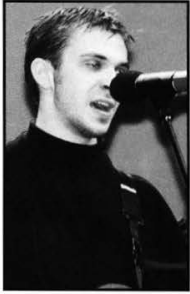

Particpating in the band Monkey Business, David McGrew plays electric guitar.

Iustint Swanson and Ray Greene dance with Brockstrect Boys and the martial arts team.

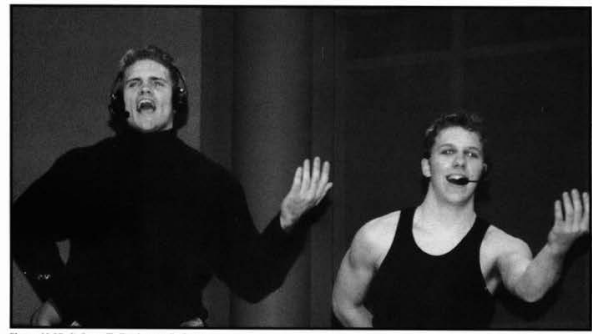


$\mathcal{N}$ oaf Ronczkowski plays cello for the first place finishers in the music category, Boys From Lawlor.

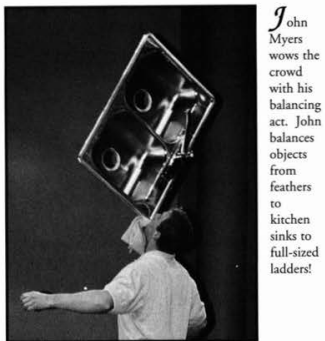

\section{"Ihe purpose of the Alpha Chi Talent Show was..."}

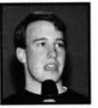

Tin Cochiref?
"... to use this night of entertainment as a ministry

to reach the unsaved."
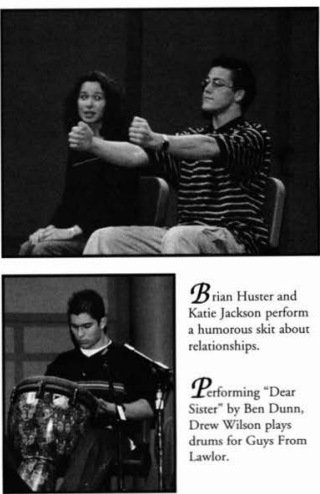

$\mathcal{B}_{\text {rian Huster and }}$ Katie Jackson perform a humorous skit about relationships.

$\mathcal{P}_{\text {erforming "Dear }}$ Sister" by Ben Dunn, Drew Wilson plays drums for Guys From Lawlor. 


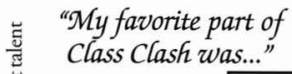

"...getting together with friends to show our class

unity and spirit."

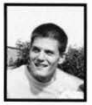

"Class Clash is a very unifying experience because it allows students to bond through competition," notes Junior Jenni Maass.

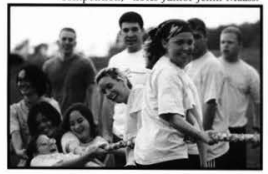

Battle of the Classes

On May 10-11, students from each of the four classes competed in the third annual Class Clash. The competition pitted the classes against each other in various athletic contests.

Participants enjoyed the good-natured rivalry between the classes. "We had some pretty good competition," said sophomore Jeremy Cunningham.

Senior Jordan Patrick noticed the teamwork involved. "It was an opportunity to compete against different classes, to get out and have a good time, and to do something different," he said.

The cance relay race was one of the more interesting events of the day. One cance overturned, and another team was doing well until a paddle broke. "It was a lot of fun," said senior Kim Ketterer.

The juniors carried the day, winning for the second year in a row. "I think it's awesome," said junior David Malwitz. "I think it says something about our class."

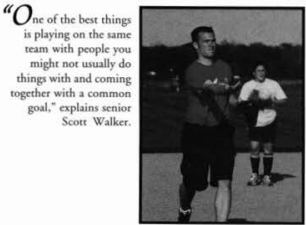


${ }^{\text {"I }}$ really enjoy Class Clash because it's an opportunity to interact with people from my class that 1 might not otherwise come into contact with," says Junior Brian Neises.

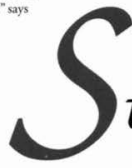

\section{get involved}

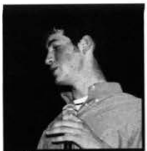

"The talent show was a great way to see the diversity and creativity represented in the student body," says Chris Hennig.

\section{Introduc-} ing one's talent

to the

student body proved to be fun and enjoyable.
The darkening of the auditorium, the anticipation of the audience, and the glare of the spotlight all added to the occasion of the evening. The New Student. Talent Show proved to be a pleasant distraction from the typical hectic schedule of student life.

The talent show took place in the Cedarville Opera House, with freshmen and transfers composing a total of eleven groups of performers. The presentations throughout the night evidenced the diversity of the students. Acts ranged from original guitar ballads to poetic oral interpretation. Each performance was unique, as students composed many of the pieces themselves. One particular performance, "Steppin' on the Clouds," sung by a female a cappella quarter, was so lively that the audience got caught up in the spirit of the song, and a wave of clapping hands and stomping feet swept throughout the building. Chuckles of amusement and bursts of outright laughter came from the audience during the lip-synch of two selections from the Broadway musical "You're a Good Man, Charlie Brown." The students performed the routine so well that the group portraying Charles Schultz's "Peanuts" gang won first place in the competition.

Judges selected winners based on performance, quality, and overall act. All the groups illustrated remarkable talent, and the judges had a difficult time deciding on the top three. The students did an excellent job of grabbing the attention of the audience and then maintaining that attention throughout the course of the evening. Cedarville's new students expressed not only their ability, whether musical or otherwise, but also their uniqueness as individuals.

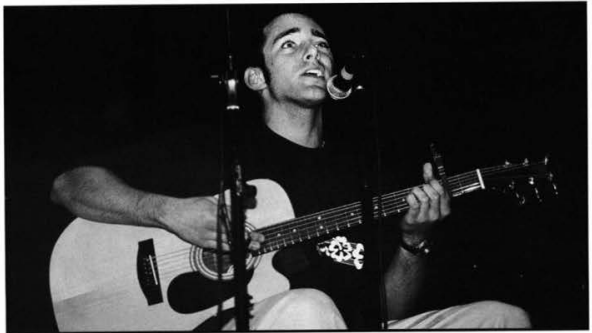

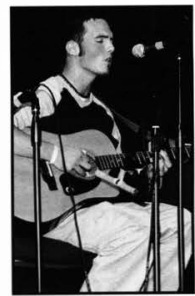

"The talent show was a great way to get some of the new people involved and give them a chance to show Cedarville the talent they are bringing in," notes Matt Bell.

Senior Michacl Dorseyemcees the New Student Talent Show.

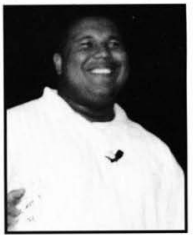

"Vot Enough" written by transfer sophomore Justin Tubbs is performed at the New Student Talent Show. 
... if we claim to have fellowship with Him yet walk in the darkness,

we lie and do not live by the truth... 


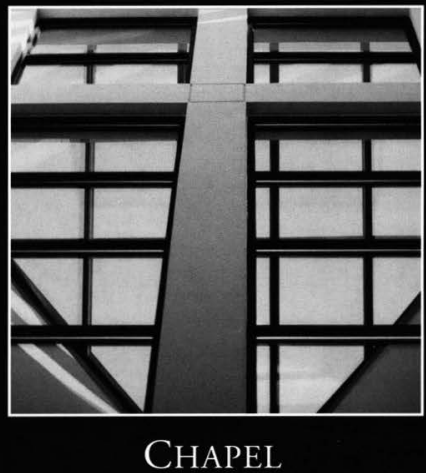




\section{Comfort}

\section{through Trials}

The Fall Bible Conference is different than the others during the year. It introduces new students to the college. Freshmen receive their first glimpse of college life at Cedarville, and transfers get to compare Cedarville and its views to other colleges and universities. To returning students, it provides a transition from summer jobs and ministries back to studying. Sometimes. returning students see a new look to the campus as the college continues to expand.

This year, the conference launched Cedarville students into a new school year with Dr. David Jeremiah was the speaker. Dr. Jeremiah has a unique relationship with Cedarville College. Not only a former student, he was also the son of Chancellor James T. Jeremiah. As a student, David Jeremiah's interests centered around working on the radio. He began CDR radioat Cedarville, which was currently one of the college's major outreach ministries. Dr. Jeremiah gave the college family some spiritual advice during a tough time.

In the first chapel service of the year, students learned how God had been working over the summer. Van and motorcycle accidents, along with the passing away of members of the college family, compelled students and faculty to turn to God. Dr. Jeremiah. preaching from the Psalms. shared his own experience with trials. He encouraged his audience to turn to God during trials, and in turn God would provide His comfort. David Jeremiah explained how hardships make a child of God better able to serve others; and though God's plan is not always evident during difficult times, His goals will be manifest.

The 1999 Fall Bible Conference was a learning and growing experience for the entire college family.
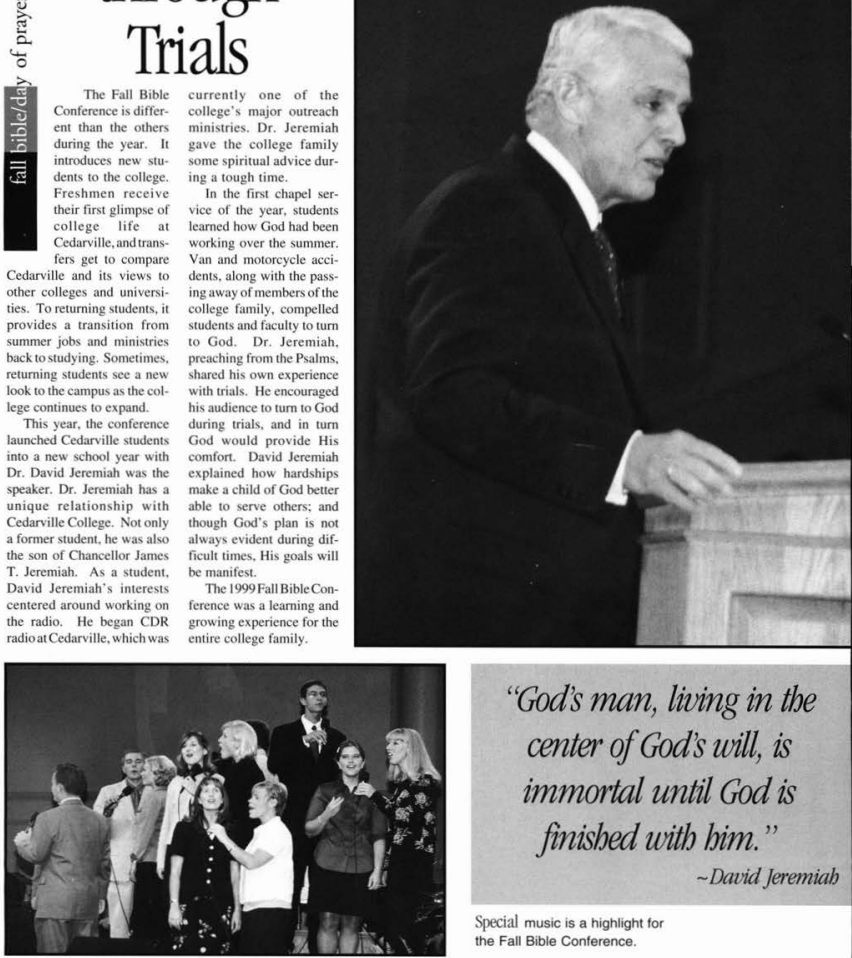

Special music is a highlight for

the Fall Bible Conference. 


\section{Focusing on the \\ Light in a New Millennium}

The dawn of a new millennium was fast approaching, homecoming festivities had taken place a few weeks earlier, and students were, without a doubt, dreading their coming midterms. These activities, combined with a regular college schedule made the 1999-2000 fall quarter demanding. Foreseeing this pile-up of events, the administration wisely chose Wednesday, October 27, 1999. as a day to calm the normal bustle of campus life and pray for the specific needs of the college. country, and worldwide current events. A unique chapel service highlighted the day. It included an amazing time of praise and worship led by Dr. Vernon Whaley, with dynamic special music by Jubilate, Lindsay Wagner presented a powerful memorized presentation of God's Word. Dr. Dixon brought a convicting message about Christ's

Dr. Jeremiah is an inspiration to students as they begin a new year. second coming and prayed with the college family. Chapel ended with a bonding time between the students as they kneeled and prayed together in groups of threes and fours.

Other prayer-centered activities took place throughout the day such as hall prayer breakfasts, staff and faculty prayer time, and advisor/advisee prayer groups. The administration, in planning this day of prayer, did well in assuring that the focus for the day was the communication with God that is unique to Christians and is sometimes taken for granted.

Rick Carter, as a freshman and new member to the campus, expressed his view of the day of prayer this way: "I believe that prayer is important, and I like that Cedarville sets aside a whole day and makes prayer the top priority."

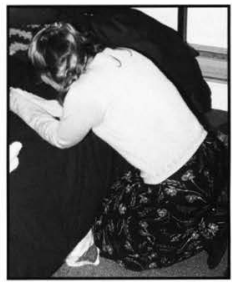

Students find time to spend quiet moments in prayer.
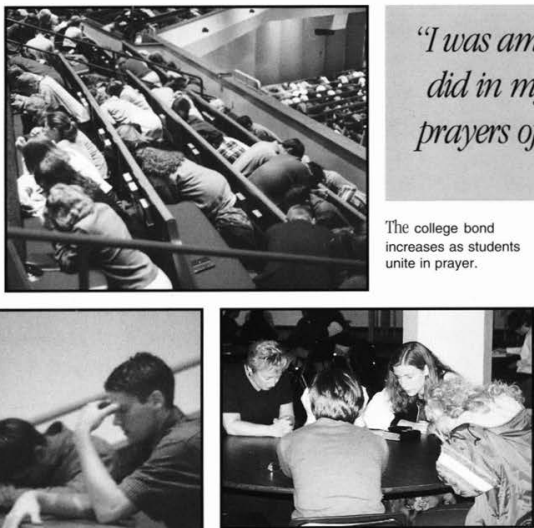

Important issues are lifted in prayer.
Coach Clark leads her advisees in prayer.
The college bond increases as students unite in prayer.

\section{"I was amazed by what God} did in my life through the prayers of my classmates."

\section{Jenny Stryker}

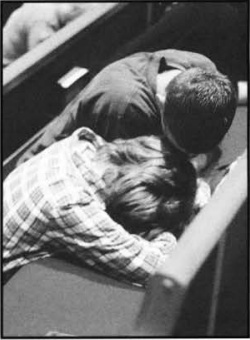

Friends kneel together to pray for daily concerns. 


\section{The First and Greatest}

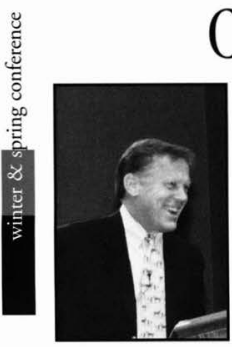

Joe Stowell always brings a healthy sense of humor to the pulpit.

Missionaries come from around the world to participate in and attend Cedarville's annual spring conference.

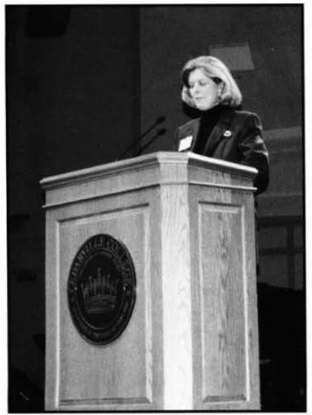

Concert chorale provides a special music performance during the conference.
Without boundaries. This is how Christ would have us to love $\mathrm{Him}$ as well as those in the world around us. In this year's Winter Enrichment Conference, Dr. Joseph Stowell, President of Moody Bible Institute, opened the eyes of the Cedarville student body to what it meant to love without boundaries. Using numerous practical examples, he made clear the many ramifications of adopting Christ's unconditional love into one's life, and nearly all students felt convicted by his powerful calls for change. "Lost people are not our enemies," he said. "Our greatest enemies are the gods we will not give up in our own lives." Indeed, students felt urged to rid themselves of those idols in their hearts that had long prevented them from lov-

ing and witnessing effectively.

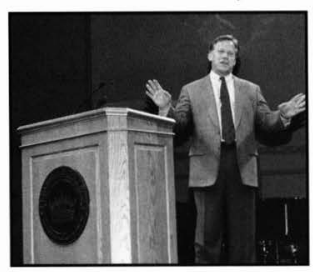

Keynote speaker Joc Stowell shares insights with the collge family on how to live for Christ. day night's message gave evidence to the power of his utterance. Freshman James Miller wasstruck by the fact that, "Dr. Stowell did not point out specific things that we should or should not do." Rather, he learned from Dr. Stowell that, "our love for God should take precedence over our love for our earthly possessions and desires." According to Stowell, this precedence will help us to determine the specifics. Certainly, everyone gained a new perspective on those without Christ from Dr. Stowell's moving stories and chal. lenges. Dr. Stowell's message will long remain in students' hearts and minds, and it will continue to impact their world view. Hopefully, we The overwhelming response to will never love Christ or our neighbors in the the altar call following Thurs- same way again.

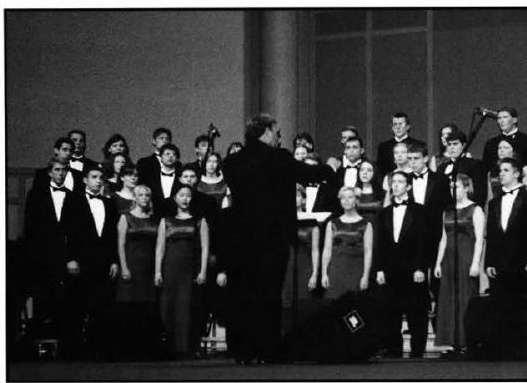



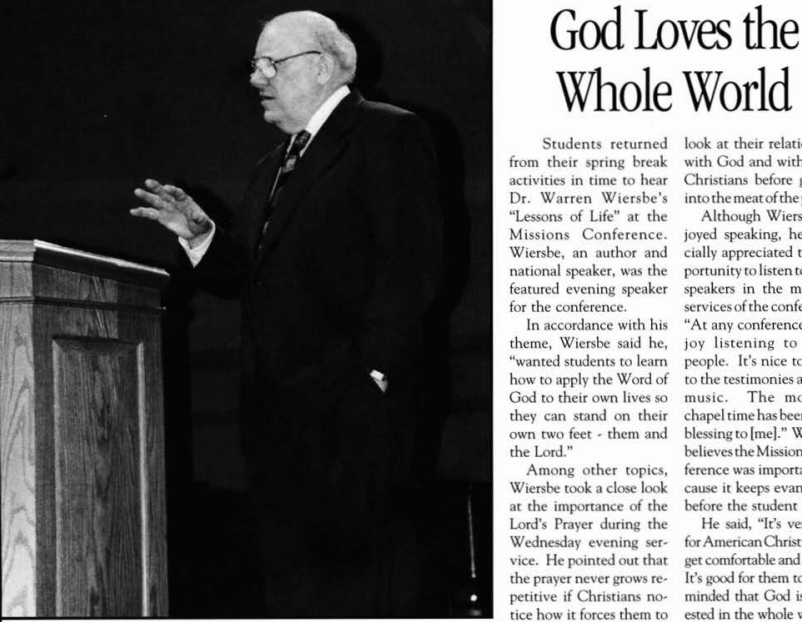

Students returned look at their relationship from their spring break with God and with other activities in time to hear Christians before getting Dr. Warren Wiersbe's intothemeat of the prayer. "Lessons of Life" at the Although Wiersbe enMissions Conference. joyed speaking, he espeWiersbe, an author and cially appreciated the opnational speaker, was the portunity tolisten to other featured evening speaker speakers in the morning for the conference.

In accordance with his theme, Wiersbe said he, "wanted students to learn how to apply the Word of God to their own lives so they can stand on their own two feet - them and the Lord."

Among other topics, Wiersbe took a close look at the importance of the Lord's Prayer during the Wednesday evening service. He pointed out that the prayer never grows repetitive if Christians notice how it forces them to services of the conference. "At any conference, I enjoy listening to other people. It's nice to listen to the testimonies and the music. The morning chapel time has been a real blessing to [me]." Wiersbe believes the Missions Conference was important because it keeps evangelism before the student body.

$\mathrm{He}$ said, "It's very easy for American Christians to get comfortable and selfish. It's good for them to be reminded that God is interested in the whole world."

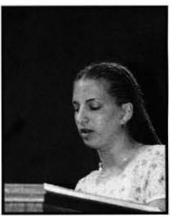

Spring conference is also a time for students to share about their spring break mission trips.

Bagpipes are a highlight of the special music and a favorite among the college family.

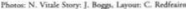




\section{Preparing Leaders}

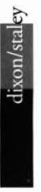

As the 1 leader of this college, President Paul Dixon could not have chosen a better topic to be the focus of his Monday chapel messages for the 1999-2000 school year.

With the influences of leaders such as former president James $T$. Jeremiah, Dr. DonSewell, and the president of Dixon's alma mater, Tennessee Temple, Dr. Lee Roberson, Dr. Dixon's path to his position of leadership was a clear one. During the year, he was the one influencing and encouraging the students to walk along the same path and to adopt the same mission in life. He was passing the torch.

Combining his own personal experiences with Biblical principles, Dr. Dixon poured out his knowledge of leadership on the student body. $\mathrm{He}$ felt that there would al. ways bea great need for Christian leadership in all venues of life. His desire was that the students make a major com- mitment to becomingChristian leaders in the twenty. firstcentury.

His messages included topicssuchas "WhoaLeader Is," challenging thestudents to take great pains to build their character so that it modeled Christ. Humility, genuine concern, a servant's spirit, a genuine love for people, intelligence, vision, integrity, and a positive attitude were just a few of the traits Dixon touched upon asvital to a leader's makeup.

Later in the year, during spring quarter, Dr. Dixon made "What aLeader Does" the focus of his chapel messages, challenging everyone to adopt a purpose or mission in life and to look to Jesus as the model mission maker (John 17:4, Luke 19:10).

As an overview of his year-long chapel series on preparing Christian leadership, Dr. Dixon said, "Being a Christian leader has always been a difficult task, but by God's grace, we can be."
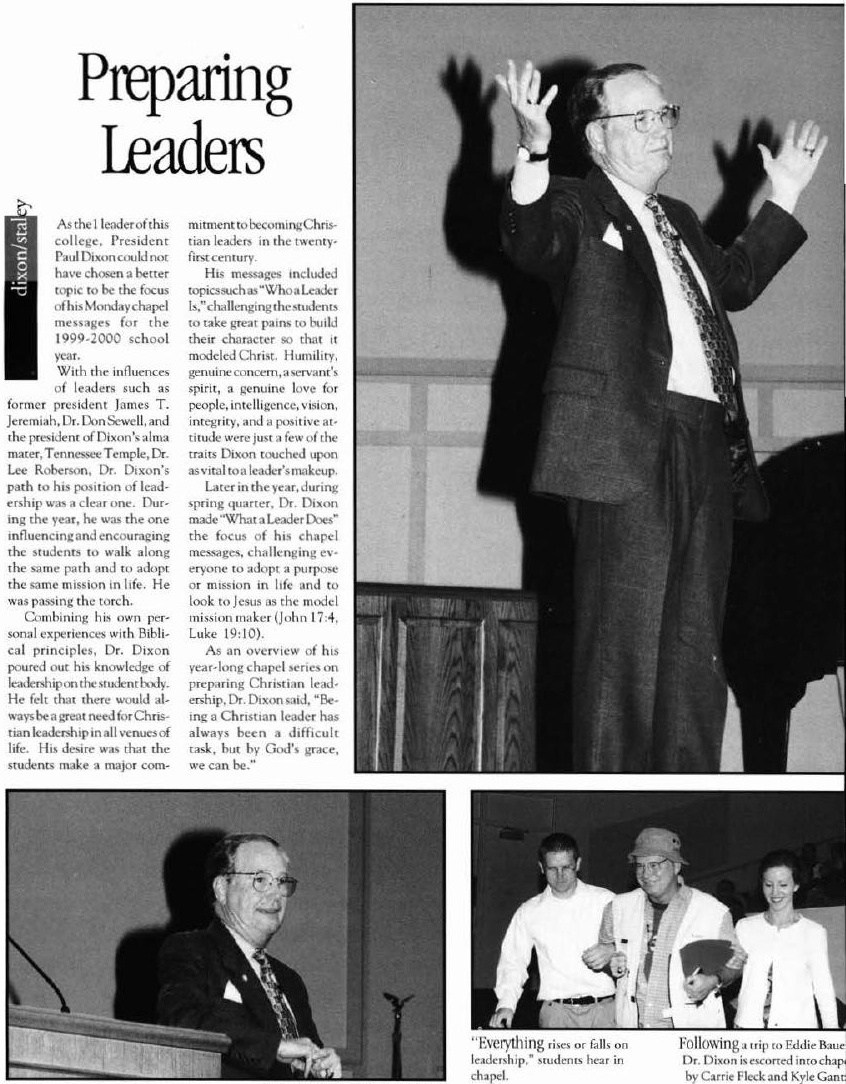

"Everything rises or falls on leadership," students hear in chapel.
Following a urip to Eddie Bate Dr. Dixon is escorted into chap. by Carrie Fleck and Kyle Gant 
"If you are going to be used of God, you must be a person of values," proclaims Dr. Dixon.

\section{Knowing is Doing}

Every year, the Bible department sponsored the Staley Lectureship Series which was held during the chapel hour in the Jeremiah Chapel. One speaker was brought in each year to share with the student body.

During winter quarter, Dr. Steven Garber came to present his series of Staley Lectures. As a professor from the American Studies Program which was sponosored by the Cosalition of Christian Colleges and Universities (CCCU), he had a platform from which to address the students concerning various issues that confront today's culture.

He held to the fact that if the meat around us is rotting(the decay of the moral fabric), it is the fault Christians who have been called to be salt and lighe in this world.

Garber wanted students to grasp the fact that just simply having knowledge stored away is not good enough. "To know is to do," he said. "...It's a step into what you believe," he added.

Since 1998 Dr. Garber had held the position of Scholar in Residence for the CCCU. He had written the book, The Fabric of Faithfulness, which was popular in both christian and secular institutions of higher learning.

In an effort to motivate students to start acting in accordance with their beliefs, Garber asked, "What part of the world has God called you to love?"

Two Cedarville students, Michael Ferrigno and Aaron Mercer, attended the American Studiesprogram during the fall semester where they sat under Dr. Garber's teaching. Michael Ferrigno commented, "Dr. Garbers lecture on Christian responsibility has both encouraged and challenged me in numerous ways."

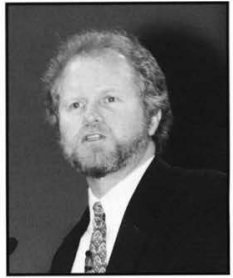

Garber highlights the priority of advancing God's truth, love, and justice.

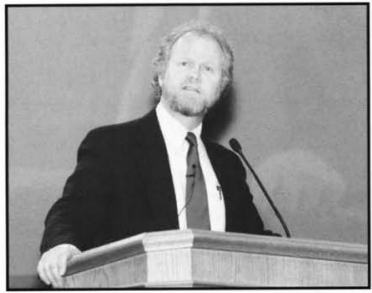

"Our knowing must reflect God's knowing, "proclaims Stalcy Lectureship speaker Steve Garber.

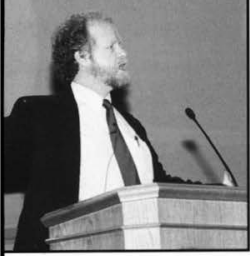

"Steven Garber's lecture on Christian responsibility has both encouraged and challenged me in numerous ways." $\sim$ Michael Ferrigno

Leaming to love what

God loves was a focus of

Garber's lectures.

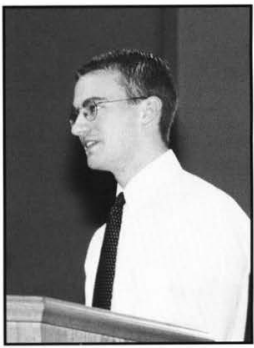

The Christian's responsibility in politics impacts Aaron Mercer while studying under Garber's teaching in Washington D.C. 


\section{A Time to Share}

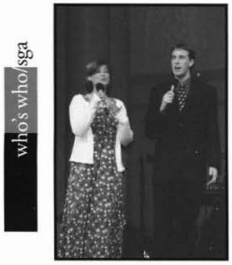

Seniors have the chance to use their gifts and abilities in Who's Who Chapel.
Jonathan Wynn provides special music as a way of sharing with underclassmen andstaff.

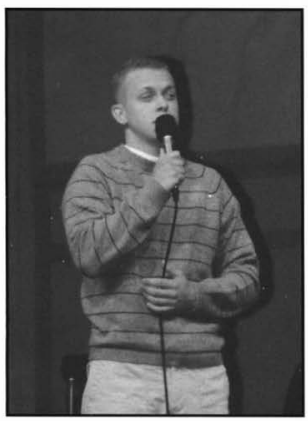

Creative skits help make Who's Who Chapel an enjoyable experience.
Fifty some seniors named "Who's Who Among American College Students" presented a chapel service to the student body with a memorable and challenging subject: living for an audience of One. The chapel also included some humorous videos of what some seniors would do if they had their wish at Cedarville.

Through roleplay, dramatiza. tion, and song, these students re. minded all in attendance that the Lord Jesus Christ is that audience of One. If $\mathrm{He}$ is not pleased, then worldly successes and earthly gains matter little. $T$ h e theme

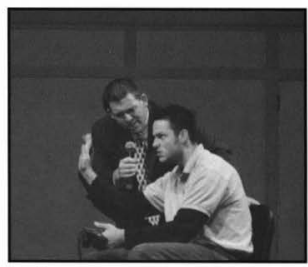

Joel Schenk attempts to take an in-depth look at a student's life here at Cedarville College. of placing the relationship with Christ," said junior Ben Gayer.
Christ above all else in life echoed throughout the presentation. The chapel ended appropriately with the song, "Knowing You." The chorus summarized the message clearly: "Knowing You, Jesus, knowing You. There is no greater thing. You're my all; You're the best; You're my joy, my righteousness..."

"I was encouraged to see their testimony on stage, but even more so by watching their lives and seeing their dedication to Jesus

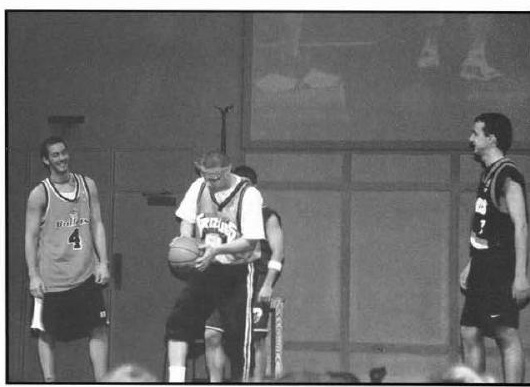




\section{honoring hard work}

\section{Faculty Member of the Year}

Chosen as the Fac. ulty Member of the Year, Dr. J. Wesley Baker could not have been more qualified for the honor. Dr. Baker had been at Cedarville since 1988, serving in the positions of News Director for WCDR Radio (1988-1992) and Faculty Coordinator for CedarNet.

Dr. Baker taught a variety of courses, including Audio/ $\mathrm{Ni}$ suals, Communication in the Information Age, Introduc. tion to Interactive Multimedia, Issues and Ethics in Electronic Media, and Mass Me. dia Law and Regulation.

Dr. Baker had continued his education since the early seventies when he graduated cum laude from Bob Jones University with a $\mathrm{BA}$ in $\mathrm{Ra}$ dio-Television Production. In 1980, he obtained an MA from the College of Journalism at the University of South Carolina. Dr. Baker carned his Ph.D. from the Department of Communica. tion at The Ohio State University in Columbus, Ohio.

Dr. Baker's extensive em. ployment history gave him real world experience to bring to the classroom. From July 1966 through 1968 , he was the News Di. rector at WLEA-FM in Homell, NY. He served as the Cistomer Service Rep. resentative at WMUU in Greenville, SC, as well as News Director and Staff Announcer from 1972 to 1975. Dr. Baker also toured as the team leader with a speech and music group called The Proclaimers in 1976 and 1977

Another one of Dr. Baker's activities was performing in several films. His first film came out in 1981 and was entitled Hudson Taylor, in which he played the part of John Burdon. In 1985 , he had the major supporting role of Dennis in Hidden Treasures. More recently in 1990, he played the part of Wes Thompson in Lost in Silver Canyom.

Dr. Baker marricd Rebecca Glenn McGuire in 1973 and together they have four children Vanessa, Nathaniel, Katharine, and Elizabeth.

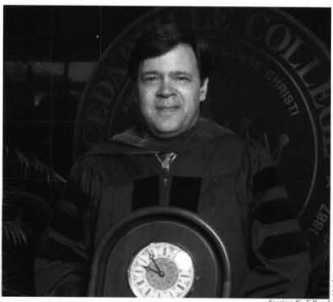

\section{Staff Member of the Year}

Selected as the Staff Member of the Year, Mrs. Sandy Entner had faithfully served the college throughout her time here. Mrs. Entner first came to Cedarville in 1955 as a student when there were ahout 120 students and less than a dosen administrators and faculty. She obtained her BA from Cedarville and her Masters in Counseling from the University of Davton. She is a Licensed Professional Counselor and a National Certified Counselor.

Before coming back to Cedarville to work in the Counseling Department, Mrs. Entnes worked ar Child Welfare in Dayton, Ohio: Hurley Hospital in Flint, Michigan, and Family Services Association in Corning, New York. When Mrs. Entmer first came to Cedarville, she worked as an adjunct professor in social work. After receiving her Master's Degree, she did her internship and practicum with Dr. Martin Clark. In 1984, she succeeded Dr. Clark as Director of Counseling. She had worked at Cedarville for fifteen yea and serving Cedarville students in personal, interpersonal, and spiritual concerns througl individual and group counseling was her ma focus.

Also while at Cedarville, Mrs. Entner hat served on the alumni council and was active with the Women's Fellowship. Mrs. Entner was very busy with all of her responsibilities but said, "Family is top priority." She had tw sons and five grandchildren. Mrs. Entner sa people were her primary interest, but other interests include her grandchildren, flowers, bicycling, hiking, and photography.

Mrs. Entmer said, "It has heen with jov ar wonder that I have watched Cedarville College become Cedarville University becat of God's hand of blessing. To have been her from the beginning makes me appreciate all that He has done." 


\section{remembering the cost of freedom}

According to Dr. Dixon, the Memorial Day Chapel service had yearly been one of the best memorial services in the nation. Each Memorial Day during chapel, Cedarville College had recognized those who served this country in the armed forces in both war and peace.

Students chosen by Dr. Robey led the memorial service by focusing on those veterans who served during World War IL. Using NBC anchor Tom Brokaw's book The Greatest

Generation, the group recited quotations from several veterans who fought in WWII. They shared the experiences of the GI, pilot, and seaman who risked their lives for their country. Senior Brenon Christofer, one of the students who led the service, quoted an Associate Press statistic that said an average of 1,000 WWII veterans die each day. Christofer said, "I'm thankful for those who gave their lives so that we could have the freedom that we do. They did not know the people who would enjoy the freedom they provided, yet they gave their lives anyway. . . Im glad that $\mathrm{I}$ had the opportunity to show my appreciation in the Memorial Day Chapel service."

The Jeremiah Chapel was crowded with students, veterans, and citizens from the area. "The Memorial Day Chapel provided a great representation of WWII veterans," said senior Christy Heflin. "I loved the WWII era music that the symphonic band played." The symphonic band, led by Mr. Di Cuirci, played while pictures from the Second World War were displayed on the screens behind the band.

Annually, the Memorial Day chapel service inspired a national pride in those who attend. The College was proud to salute those who have served in the armed forces to make this country free.

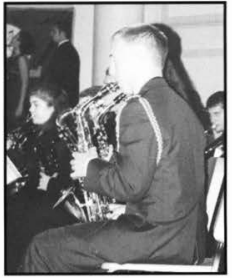

Members of the symphonic band liven up the chapel with famous marches.
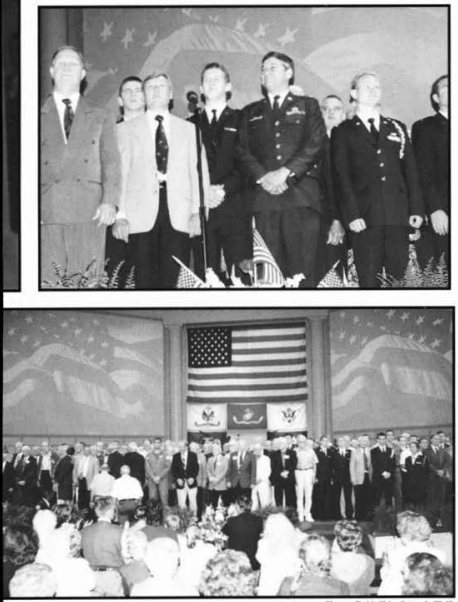

Men and women from all over the arca come to celebrate this Memorial Day through singing and testimony.

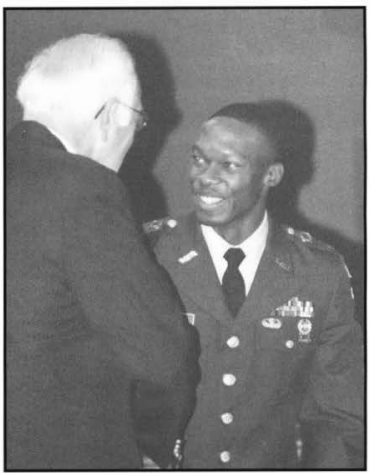

It is always amazing to sec how many service individuals are part of the Cedarville College family.
Thanking veterans and currently serving men and women is the focus of the service. 


\section{recognizing diligence and perseverance}

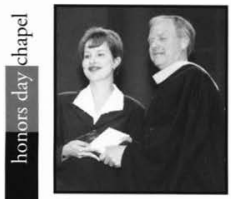

Sharon Wickhom receives the Library Science Scholarship Award.

This award recognizes Ashley Barnes for her Comm. Arts work.

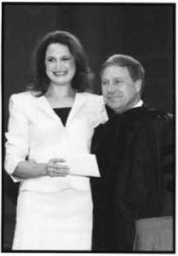

The Academic Honors Day Chapel recog. nized students who had achieved academically and spiritually. Recipients included stu. dents in every department. "It was a real honor to receive an award," said junior communication arts major Amanda Gillespie. "It meant a lot to me because it was a recognition of working hard." Junior Mike Ferrigno, who received the Joseph G. Halsey award, com. mented, "I felt honored. Dr. Halsey's in. vested a lot of time and energy in his stu. dents."

Honors Day Chapel was held on the Friday of Parents' Weekend, as tradition, soparentscould see their children accept awards. "It was exciting for me and my mom," said Aaron Mercer. "I was thankful to God because I sure didn't get it by my own power."

Senior Wes Tillet received the Hugh T. Hall Memorial Scholarship in Bible. "It was really humbling, an unexpected symbol of hard work and what God's done in my life," he said. Bible major Scott Lehr received the David G. Canine Award. Lehr plans on attending Dallas Theological Seminary. "Td love to teach Bible in a public high school or secular university," he said. "My goal is to share Christ with people."

SeniorLonnie Nolt received the Nehemiah Engineering Award. He overcame significant

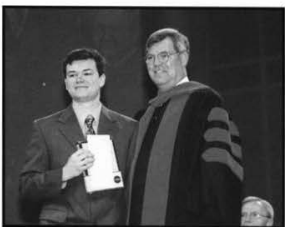

JaSon Hilgeman was one of two recipients of the obstacles in doing so, managing to graduate on time despite an accident in the fall that cost him his leg. "It wasn't easy," he said. "But I was pretty determined. and I tried to keep a good attitude about it." Nolt's accident has changed his whole career path. "l'm going to design prosthetics at the Ohio Willow Wood Company," Nolt said. "I'll be dealing with amputees all the time. Hopefully, since $\mathrm{Im}$ an amputee myself, $\mathrm{Pll}$ be able to design prosthetics better because l'll know what I need."

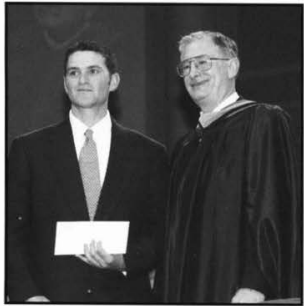

Nicholas Miller is honored by the Boyd Accounting Award.

Dr. Elmore awards Jack Barben the Zondervan Greck Award.

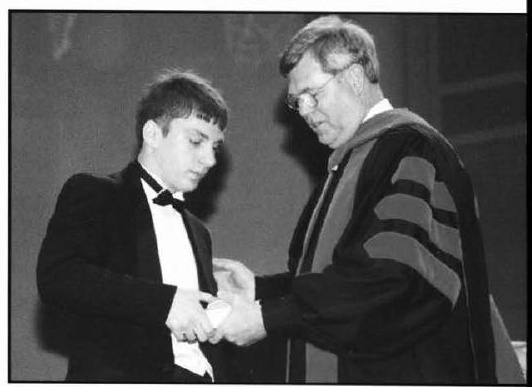




\section{Senior}

\section{Chapel}

As the year came quickly to a close for the members of the graduating class of 2000 , many events transpired, signaling theend of their time at Cedarville. Onesuch event was the Senior Chapel. During this annual chapel service, the seniors shared with the student body the various lessons that they had learned during their four years as college students.

The service began, after a rousing cheer by the senior class, with a skit that had a set. up similar to a talk show. During this skit, the "host" and her "guest" discussed what life had been like for the seniors during their freshman year.

The next several members of the class shared some of the advantages they possessed over the underclassmen, including the ability to smuggle food out of Chuck's successfully. Todemonstrate this ability, they showed a video they had made of the ingenious stealing of an orange from the cafeteria.

Following was a reader's theatre that articulated the struggle that many of them had faced that year with the question, "So what are you doing next year?" The students involved in this aspect of the chapel service shared bow they had wrestled with the uncertainty of what life after college would hold for them and the conclusion that they had come to. This conclusion was that God is in control, and that he will not only go with them, but he will go before them as well.

Some of the seniors also shared how God had shown his goodness to them during their years at Cedarville College. Although they had faced difficulties in their individual lives, theywere able to proclaim sincerely that God truly is good.

As the service drew to a close, all of the seniors gathered on the platform to conclude with the song, "You Have Been Good."

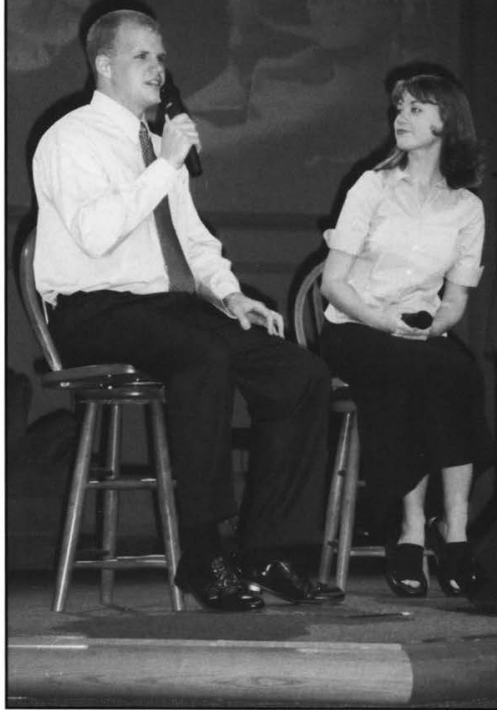

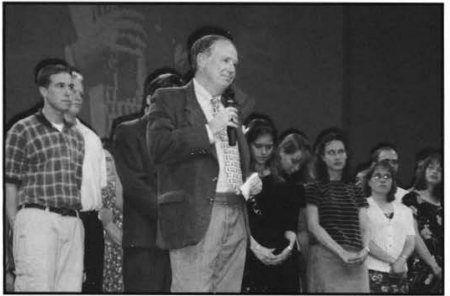

56

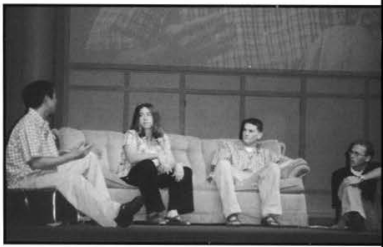

After singing the class song, the seniors are joined on stage by Dr. Dixon.
Using short skits as a tool, senio relate some lessons that the learned during their college caree 


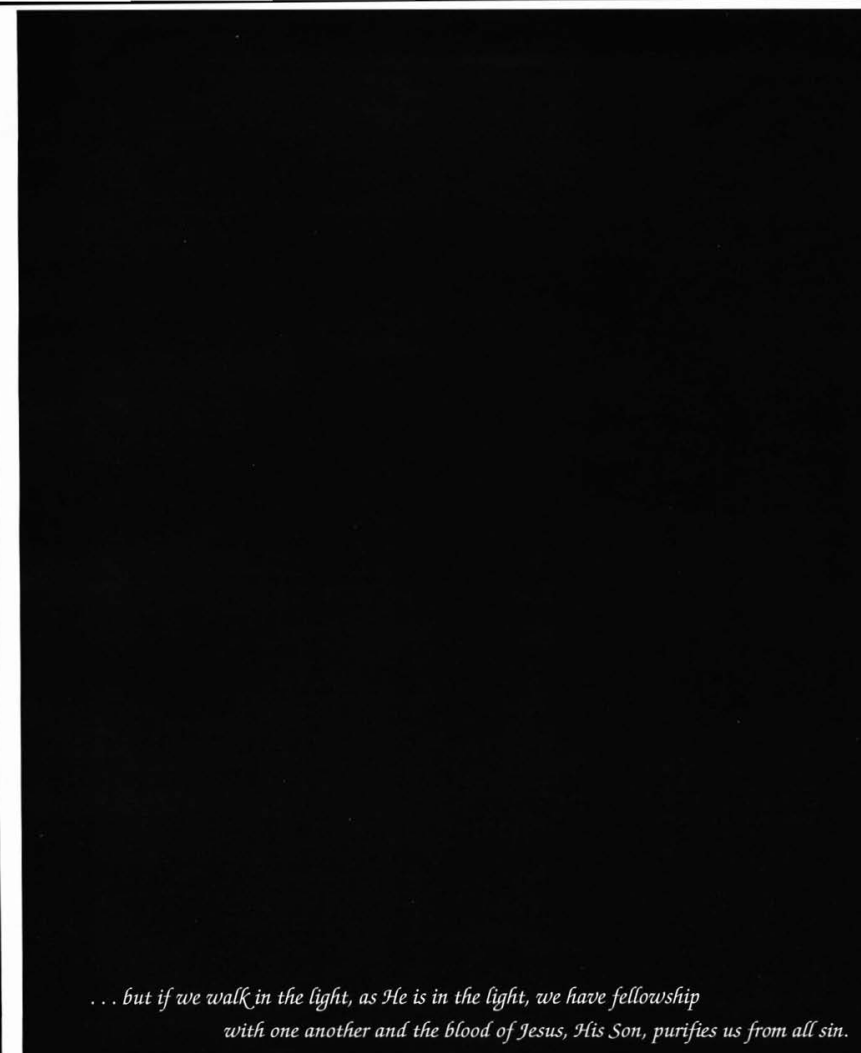

with one another and the blood of Jesus, His Son, purifies us from all sin. 


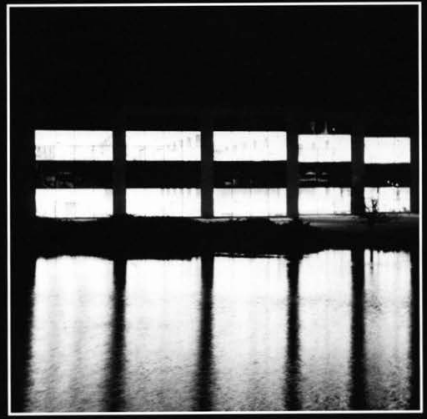

ACADEMICS 


\section{Board of Trustees}

$1999-2000$

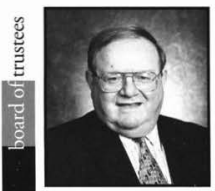

Eugene Apple

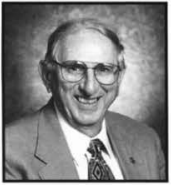

Francis Bresson

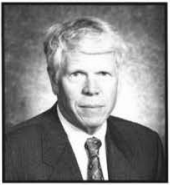

James $D_{e} V_{\text {ries }}$

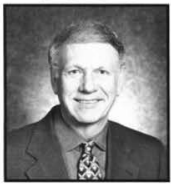

David Graham

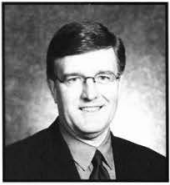

William Bernard

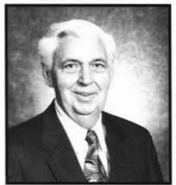

Gilbert Brueckner

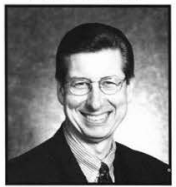

Daryle Doden

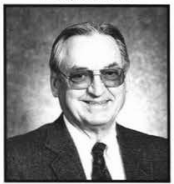

Roy Guenin
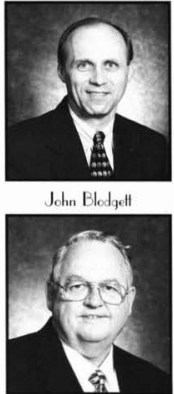

James Carraher

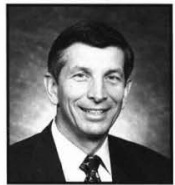

James Enqelmann

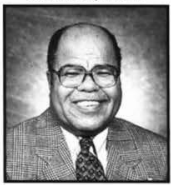

E. L. Hawkins

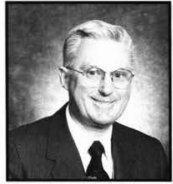

William Bolthouse

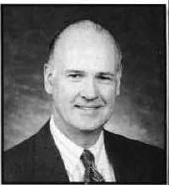

William Commons

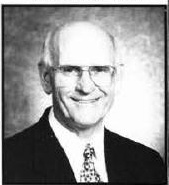

Joseph Godwin

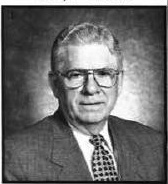

Jack Jacobs 


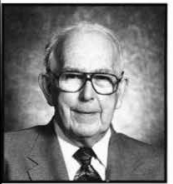

James T. Jeremiah

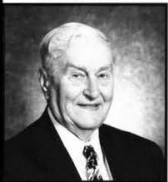

J. Dale Murphy

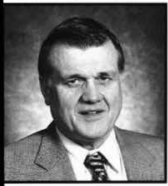

Lome Scharnberg

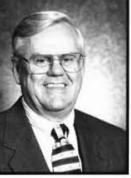

Albert Slevens

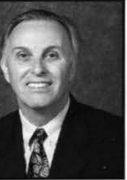

David Warren

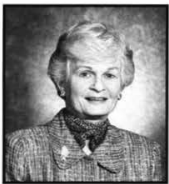

Ruth Kemplen

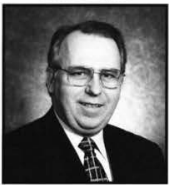

Randy Dattern

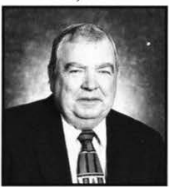

Bill $S_{\text {milh }}$

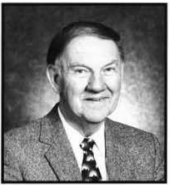

Denalf Tyler

Yol piclured:

David Gewer

and Donald Moran

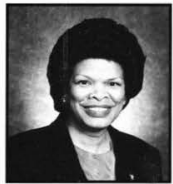

Deforia I ane

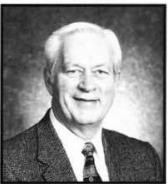

I yon Rogers

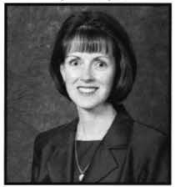

Sherri Smith

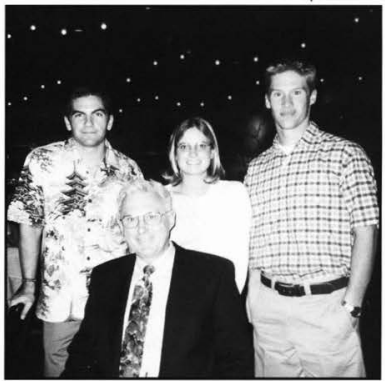

"The integrity of $\mathrm{Mr}$. Bolthouse is something that I greatly admire," says Jeremy Jeffries, pictured with fellow Californians Chrissy Redfearin, William Bolthouse, and Tim McCorkle.

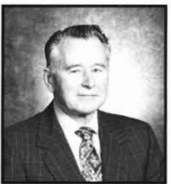

C. E. Gene Miller

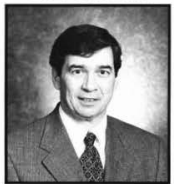

Willim Rudl

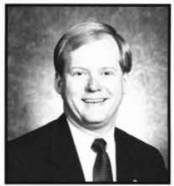

Bruce Sparts 
Administration

$1999-2000$
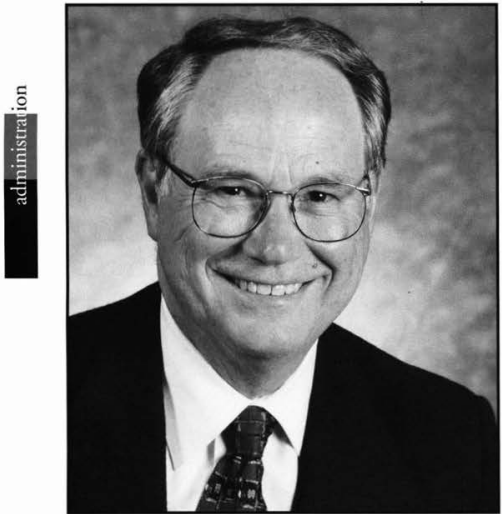

Daul Dixon, Ed. D.

President

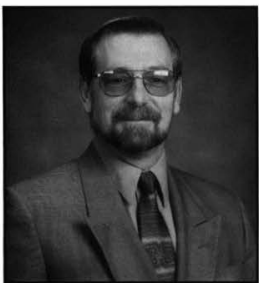

Jack Campbell, M.A.

Dirextor of Institulional Reserach

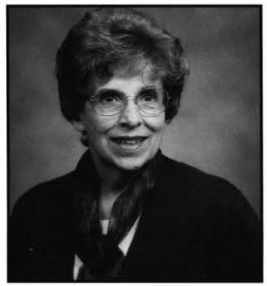

Sandra Entner, M.S.

Directer of Counseling Serrices

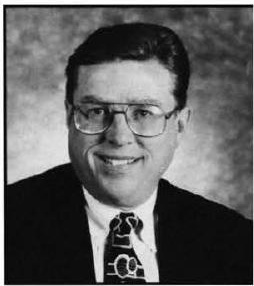

John Anqlea, M.B.A.

Vice President for Business

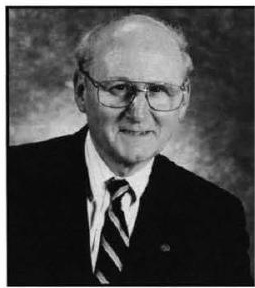

Donald Rickard, M.A.

Vice Prosidemil for Humon Services

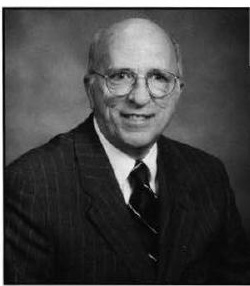

Lew Gibbs

Direder of Carer Services 


\section{Faculty}

School of Engineering, Nursing, \& Science

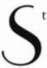

triving for excellence in all areas from character to the classroom, the department of Engincering, Nursing, and Science served to prepare students for their futures as professionals.

The infamous cardboard canoe race across Cedar Lake introduced first year engineering students to the program. Hands-on experiences, such as the pre-med. students' lab work and the nurses' clinicals, aided in acquainting aspiring engineering, nursing, and science majors with their fields.

Because of how well the department prepared these students beginning with their first core classes, other institutions of higher educa-

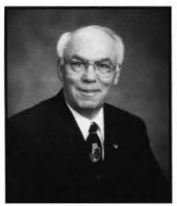

Danid Welet, Th.D. I.

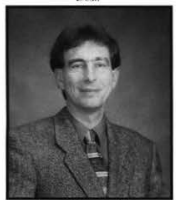

Demis Flentąe, Dh.D.

Chair of Science \& Moth Departanenl tion, as well as major corporations highly respected Cedarville's School of Engineering, Nursing, and Science.

Jay Talladay, a senior Mechanical Engineer, said that "major corporations appreciate the amount of time the students must spend in the lab. Although I didn't appreciate labs at the time, the two internships I've had have been in a lab setting, and my experiences at Cedarville prepared me well."

While the school required topquality performance from the students in each department of ENS, the goal to bring honor to the Creator and make a difference in the world was of utmost importance.

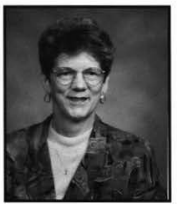

Irene Alyn, Ph.D.

Chair al Pursing I, pathent

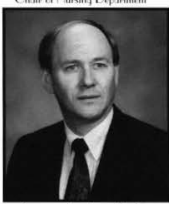

Limence Zavedney, Dh.D.

Chair of Enginecring Departenenl

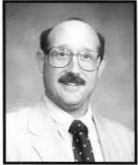

Rebet Chasnes, Dh.D. Enqineering

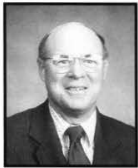

Harwed Hegro. Dh.D.

Enjexering

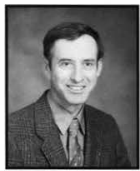

F.let I armivere, M.S.E. Fnpinusing

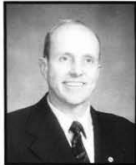

Kaith Francis, Dh.D. Enginereing

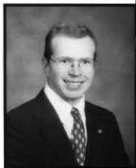

Clint Kohl, Ph.D. Eiminering

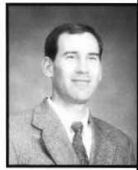

Samiel SanGreqery, M.S Einginerrimy

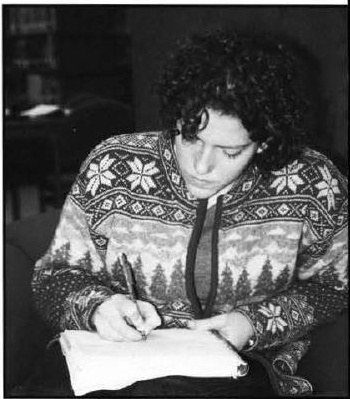

Sludenls take advantage of the library to get studying done. 


\section{Faculty}

\section{School of $\mathrm{I}$ ealth and Human Derformance}

Dr. Ager and Dean Rickard pass out 4.0 mugs to students in chape

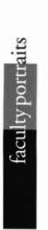

T he School of Health and Human Performance was home to the Depart. ment of Exercise and Sport Science as well as the Athletics Department. Currently 250 athletes participated in the college's 14 different varsity intercolle. giate sports. The Department of Exercise and Sport Science served 72 students who took part in various athletic activities throughout their time at Cedarville College.

The School of Health and Human Performance offered its students a quality education. The School of Health \& Human Performance sought to provide a program that would promote spiritual, physical, and intellectual development of each individual through the medium of physical and intellectual activity, within the academic offerings and participation on athletic reams. There was a quality faculty and staff to ensure that the education the students received was one of excellence.

The Dean of the School was Dr. Don Callan. He had been at Cedarville for over 40 years. Dr. Callan was also the men's basketball head coach from 1960 . 1995. John McGillivray was the chairman of the Department of Exercise and Sport Science. He also was head coach of the women's soccer team and an instructor.

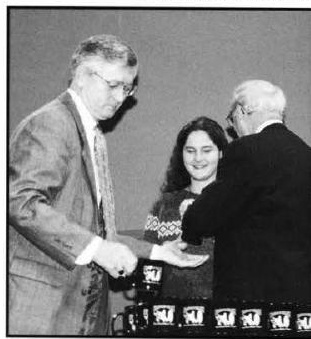

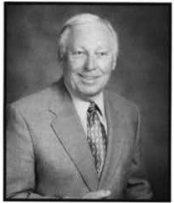

Denoll E. Callan, Dh.D Dom

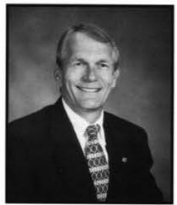

Jethe MGGilliway, M.S Chor d furise and Syat Sorres

\section{Chrissie Fretts} takes rime out of her day to study in the library.

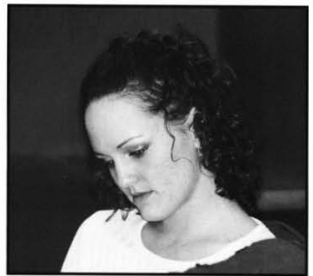

Coach Norris Smith helps out at a cross-country meet. 


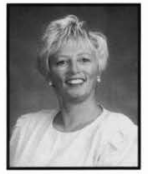

Tersa Clat, M.A

Euroue and $S_{\text {pel }}$ Science

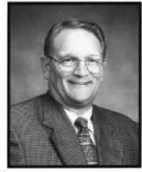

P.ten Ducharit, M.S.

Erencies and Spoed Science

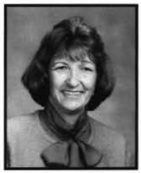

Kulluy freme, MS.

Fancie and Speol Seinnace

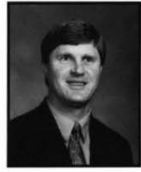

Evan Follwig. Fh.D.

Fureine and Speat Sciense

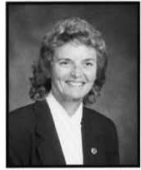

Fumela Jchore, DL.D. furrix and Spat Scanere

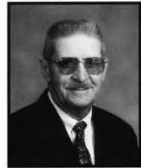

Elin King, M.EJ.

Furcie and Sperl Sciene

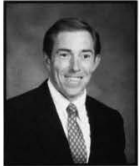

Poul Oratiard, M.A. Exereice and Sporl Srience

Library Faculty

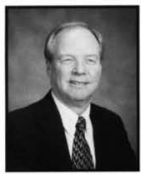

Lunn Eract, M.L.S. Litromy

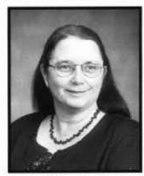

Julifh Bodly. MA. Librury

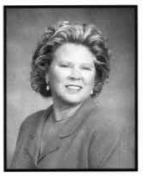

Janice Besma, M.I.S. Litrary

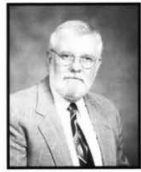

Call Branden, M.A. Lerary

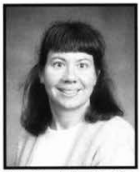

Julie Drandoffi, M.L.I.S. Litrony

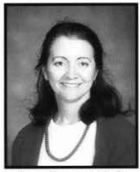

Tenys Fanced, M.L.S. Litrory

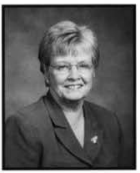

Joyce Digm, M.S. Litrary 


\section{Faculty}

School of I umanities, Fine A A ss, and Bible

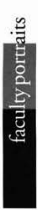

T

heSchool of Humanities, Fine Arts. and Bible was one of four schools at Cedarville College. There were

4 departments within the school. The de. partments of Biblical Education, Language and Literature, Communication Arts, and Music expanded the educational horizons of Cedarville College. There were 800 students enrolled within these four departments in 19 different majors in the School of Humanities, Fine Arts, and Bible.

Theschool put together a mission statement to help show their desire and goals. It said, "The School of Humanities, Fine Arts, and Bible exists to facilitate the disciplines ofliterature, philosophy, com. munication, performing arts, visual arts, music, and Biblical teachings to model the integration of faith and learning."
Along with the other four schools in Cedarville College, the missionstatement of the School of Humanities, Fine Arts, and Biblical Education was really an ex. pansion of the college's mission statement. The school sought to provide a quality education to its students.

Dr. Jack Riggs was the dean of the School of Humanities, Fine Arts, and Bible. He was a professor of Bible at the College. He had been at Cedarville since 1967. The Chairman of the Biblical Education department was Dr. Floyd Elmore De. James Phipps was Chairman of the Communication Artsdepartment. Mr. Ed Spencer was the Chairman on the Lan. guage and Literature department. The Chairman of the Music department was Dr.James Colman.

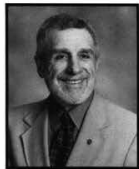

Dowid Drullinger, D. Min.

Eitlical Ejucation

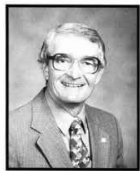

Robert Gromanh, Th. I Biblical Educalion

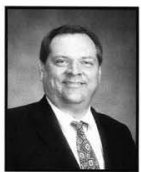

Steve Jansen, Th.M.

Bitlical Educalicn

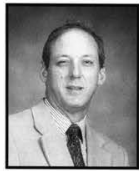

J. Midad Lepe, Ph.D.

Conrnamicaticn Adts

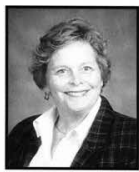

Kurt Mereland. M.A

Cemumunicalien Aok

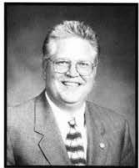

James Kraged, M.A.

Communication $\mathrm{Ash}_{\mathrm{H}}$

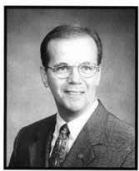

Dovid Retey, Ph.D.

Communicalon Aik
Margared Wheeler, M.FA Communicalion Ads

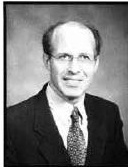

Daniel Fslos, $\mathrm{P} h, \mathrm{D}$ Bithical Education

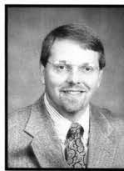

Thicmas Hulchison, Dh.D Bittical Eduration

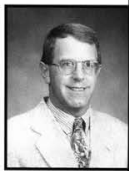

Davit Marres, Fh.D. Bitlical Education

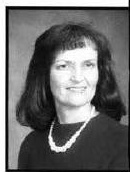

Mischelle Melntostr, MA Communicglion Arts

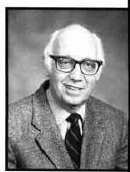

Rangrend Eartholenew Dh.1 Languaze and Literolues 


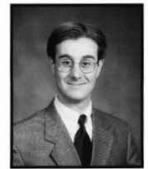

Scot Colloun, Dh.D.

Langesge and Lileralure

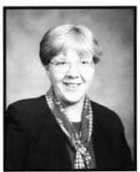

Burbors Leoch, Ph.D.

Lanquape and Literalure

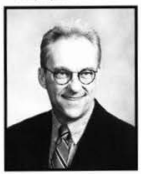

Terry Chamberlain, M.A.

Menie

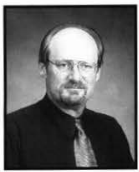

Michal D. Guiri, M.M.

Mesic

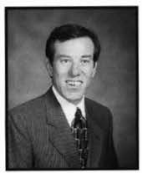

Charles Pagnard, M.M. Meric

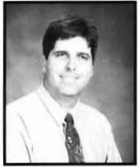

Donid Clarl, M.A

Languse and literalurs

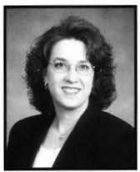

Cynthia Messer, M.A.

Lequese and Liferalues

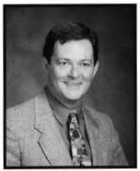

Charles Clesenger, D.M.A. Mink

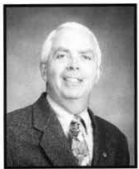

Davit Matsen, Dh.D. Muric

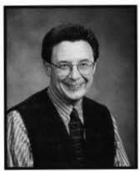

Venen Whakey. Ph.D. Murer

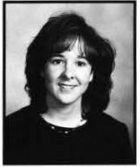

Chetal Dowis, M.A.

Lanquage and LAcolue:

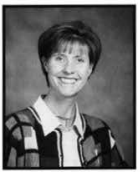

Machalle Weed, M.A.

Lonquage and Itoratare

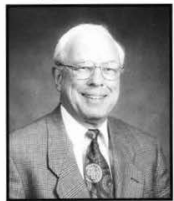

Jad Rigs. Th.D.

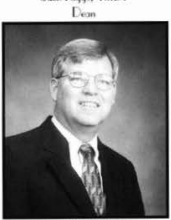

Ployl Hmese, Th.D

Chair of Bitlical Flucation

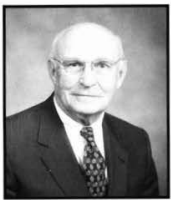

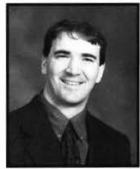

Deasld Dearicuth, Pl. D.

Lampoxe and Itemalues

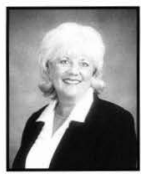

Cones Anterwen, M.M.

Music

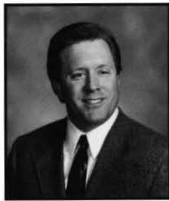

James Collman, Dh.D.

Chair of Musir

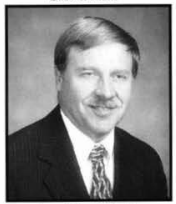

James Phipps, Dh.D.

Clvair of Commanication Asts
Sandra tame, MA

Languape and Liknalue

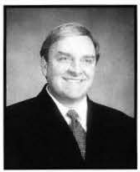

Liple Andenen. Dh.D. Music

Edward Spencer, M.A.

Chour of Lanquaze and

Liferohare 


\section{Faculty}

School of Social Sciences and Professional Studies

T he School of Social Sciences and Professional Studies housed four departments: the Department of Social Sciences and History, the Department of Psychology, the Department of Education the Department of Busincss Administration.

Dr. Merlin Agerpresidedas Dean of the School of Social Sciences and Professional Studies. Dr. Ager receivedhis Ph.D. from the Ohio State University in 1967. He was at Cedarville from 1964-75 and resumed in 1978.

Six professors including Dr. Stanley Ballard as Chairman taught in the Psychology Department. The department offered majors in psychology and applied psychology and a minor in psychology.
Dr. J. Murray Murdoch served as the Chairman of the Department of Social Sciences and History. Dr. Murdoch was also the college historian. The department had ten professors teaching students workingtowardeithera B.A. or a B.S. The department offered ten Bachelor of Arts majors and one Bachelor of Science major. It also offered five minors.

The Department of Business Administration offered seven majors and five minors. The department stated that its purpose was "to facilitate the development of business leaders who are personally discerning, professionally competent, and who will influence their organizations, professions, and culture from a Biblical world view."

Senior Jackie Smith said, "The classes are challenging, which make you think and analyze specific situations." Professor Marinus Hazen was the chairman of the department

The education department, chaired by Dr. Philip Bassett, offered programs in teaching earlyand middle childhoodeducation, adolescent and youngadult, multiage licensure, and endorsements in computer/technology and teaching english as a second language.

Senior Jennifer Tong said that in Cedarville's education department, "the difference is in the professors." She said because of the department, "I am very confident to begin teaching. The knowledge and guidance that 1 have gained through the education department will be invaluable in the classroom."

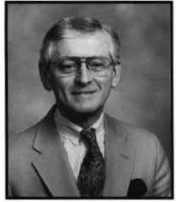

Merlin F. A yes, Dh.D. Dean

Students get hands-on experience while at Cedarville.

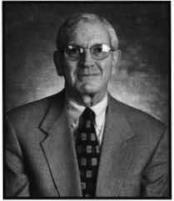

Stankey Eallard, Dh. D.

Chair df Prychology

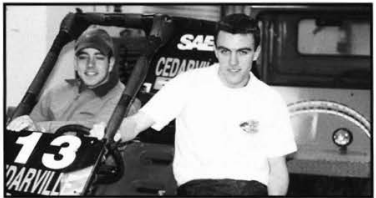

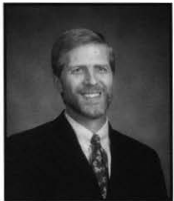

Philip Baselt, Dh.D. Chair d Edecalice

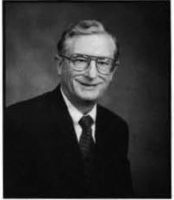

Marines Haxen, M.B.A.

Choir of Business Administration

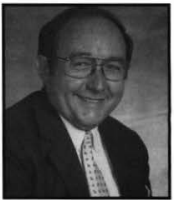

Murray Murdach, Ph.D. Chair of Social Science and History 


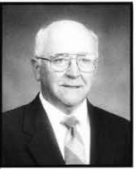

Fichard Baltwin, Dh.D

Eusiness Administration

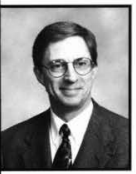

Dovid Schmidt, M.E, A.

Eusiness Administralicn

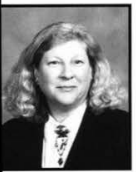

Cand Fues, MA Etucatice

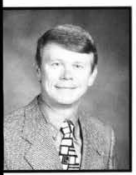

Charles Dilph, Dh.D. Dsydudogy

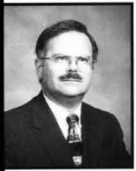

Kein Sims, Dh.D. Secial Science and Hiakery

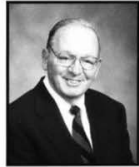

Cliflord Fanetil, D.B.A.

Buines $\mathrm{A}$ dmenoleticon

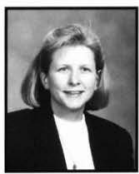

Suson Terkiken, M.A.

Euxiess Adninistraton

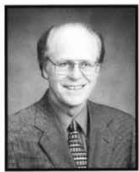

Dwape Frank, Ed.D. Educalion.

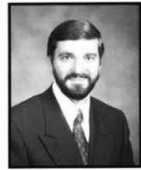

Mirtad Firmin. Dh.D Dracticlogy

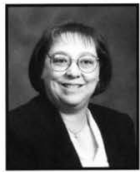

Suwan Waraer, M.A.

Secial Srienes and Hukery

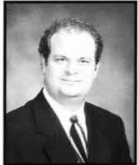

Jelfary Fancelt, M.B.A.

Business Adminisfration

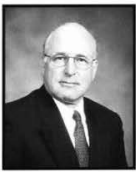

Renald Waller, D.B.A.

Eusiness Administratican

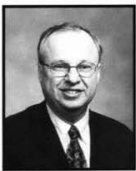

Timelly Frolon, Ph.D. Education

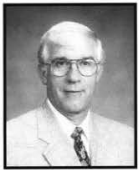

Joseph Halsey. Dh.D.

Secial Science and Hislery

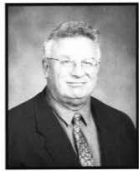

Robert Wizains, Ph.D.

Social Science and Hisiory

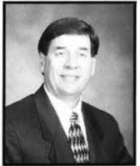

Jche L.Flanc, M.B.A.

Business Almainidrolion

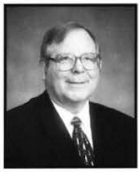

Omer Bonenteryer, D.F.

Fducolion

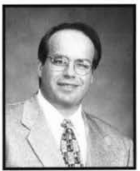

Knir Wimbon, M.Ed. Fluestien

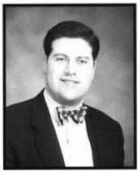

Gesen Meanctians, Dh.D. Sorial Seience and Hislem

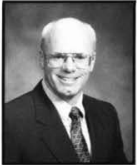

William Ragle, Ph.D.

Bonimw Alnainulration

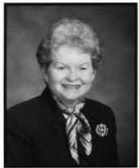

Solly Casile, Fi.D.

Education

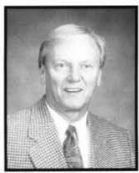

Deterl Atbar, Ph.D. Puschelesy

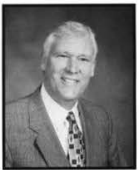

Retert Dom, Dh.D.

Sccial Soience and I istory 


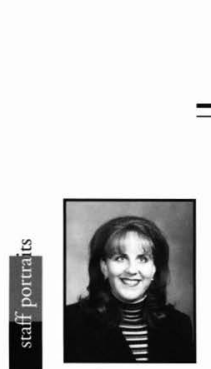

Lori Andersen

$\mathrm{Driml}_{4}$ DD

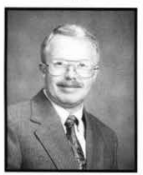

Ron Bater

Dhere. Dlent Operabeen

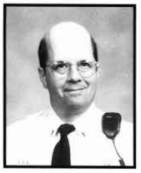

James Bawersox

Coespus Folte Soldy

\section{Staff}

$1999-2000$

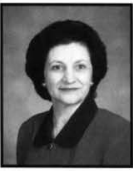

Frances Andrews

Litron

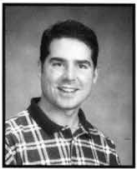

Ifff Bote

Campar Achedes

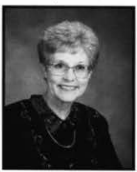

Connie Brats

Dout CAta.

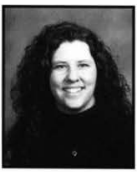

Amy Bulton

Ereakni Grution

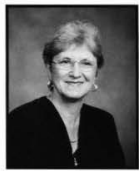

Ruth Ager

Counclian/Atwiver

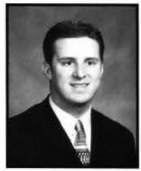

Jascon Alwell

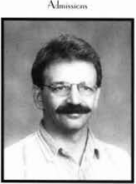

Roterl Bield

Fequed Phal Oreatem

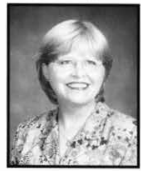

Pamela Bromer

Bate

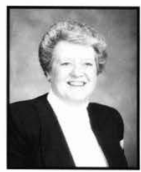

Fran Camptell

Acotanie Smios

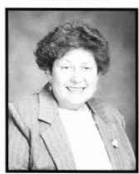

Judith Awsbdy

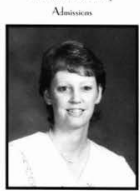

Corel Bliss

Dram Teans

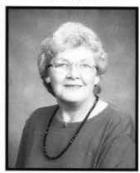

Margaret Burrichler

$$
\text { Cuitedles; CHes }
$$

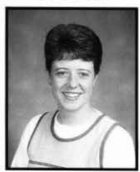

Shannah Camptell Litrary

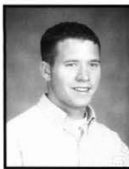

Josh Amos

Lomber DD

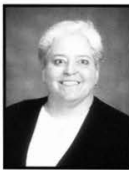

Michal Bader

Libsery

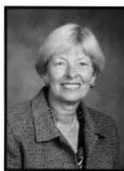

Pelen Blumenstock Aut. to Drenident

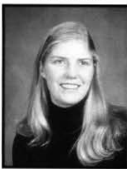

Gingar Butler Cones Senves

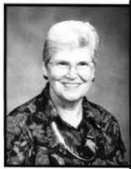

Aberla Carr Butilere 


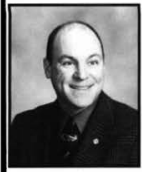

Jim Cale

Mese Iean

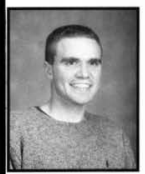

Gate Custer

Compaler Nined

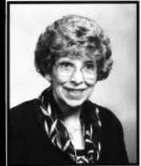

Sandy Entner

Cranalin!

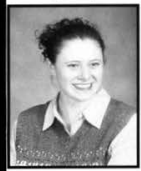

Chrissy Foullner

Eakroal Relatiens

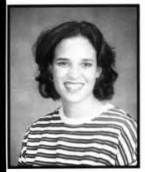

Volla Fisher

Cr... Somen

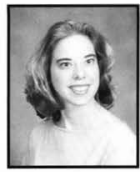

Rathel Chantler

Adminsicas

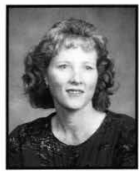

Terry Deleng

Hhang

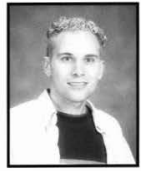

Kerry Estes

Dutle Dedilem

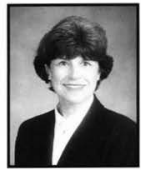

Susan Faulkner

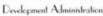

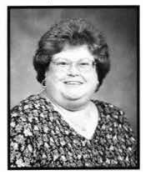

Nancy, Fissel

Conbullar's Cflis.

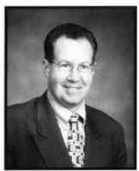

James Clark

Adminiens

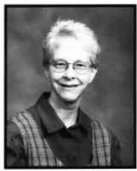

Linda Divan

tituran

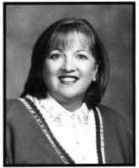

Jeyce Fartow

Drant Assislate

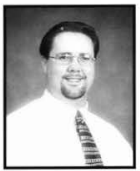

Sam Finney

Eviluere

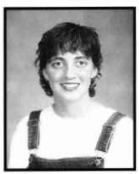

Noelle Follman

fat DI

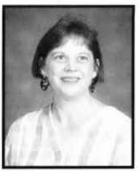

Kay Clark

Dallewen Clene

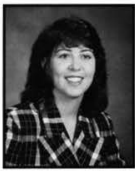

Sharon Edstein

Mine leams

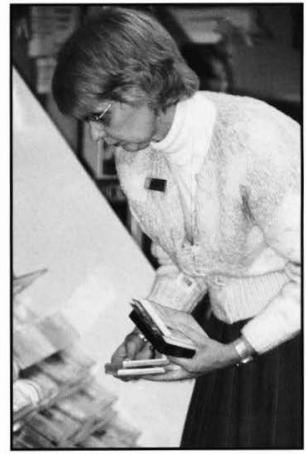

D. dicaled bookstore staff
member, Julie Olin, stocks

D. dicaled bookstore staff
member, Julie Olin, stocks

the shelves.

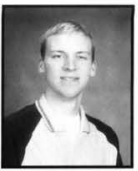

Daniel Clingan

Duth Redilim

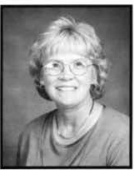

Damela Himore

Iducalica 


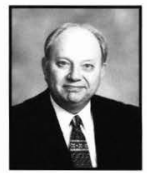

Daul Gathany

Brootent Cransteras.

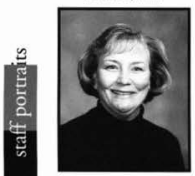

Janice Greenuced

Enpineriona

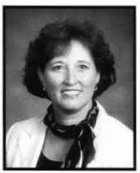

Card George

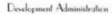

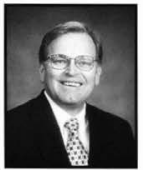

John Hess

Fimasial A I

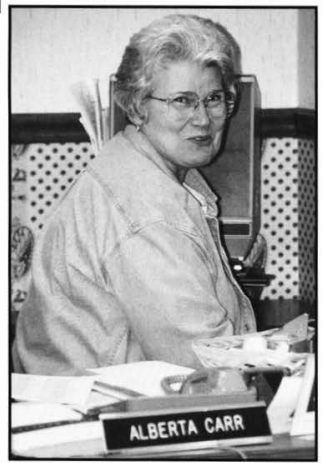

Employed at the Cedarville College Bookstore since 1978, Alberta Carr enjoys working as a book and Bible buyer. She is aware that books open new ideas and horizons to the reader.

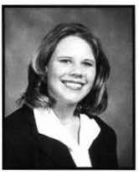

Kristi Gleasen

Dulle D.tuken

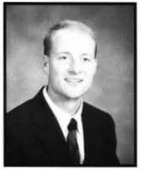

Dovid toeck

Broderad Cpenglient

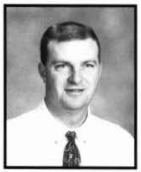

Greg Huqhes

Aldete foctikes

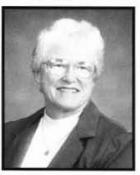

Joyce King

VPfir. Fumion

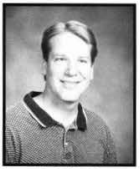

Mark Kardic

Desekpument Admiandrolen

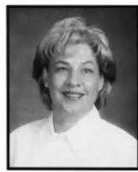

Diane Getholls,

Litray

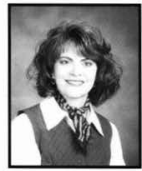

Mendy Ioltz

Ceru Cealer

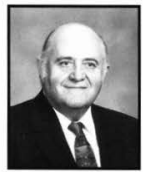

Leigh Hunt

Ner. Cendruetire

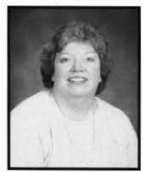

Tei King

Elucalom

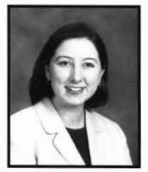

Lori Lamplen

Fimand Ai!

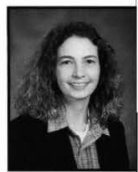

Charily Grahame

Alomen

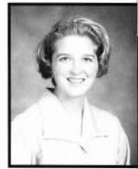

Krislin Hoover

Finneial A:

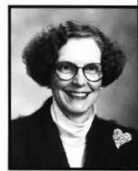

Murtho Koencher Dollowen Clines

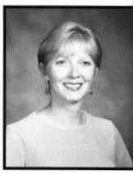

Reberca Kirby

Dallesen Chir

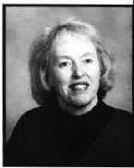

Dolricia Lee

Coulnater, CAtio 


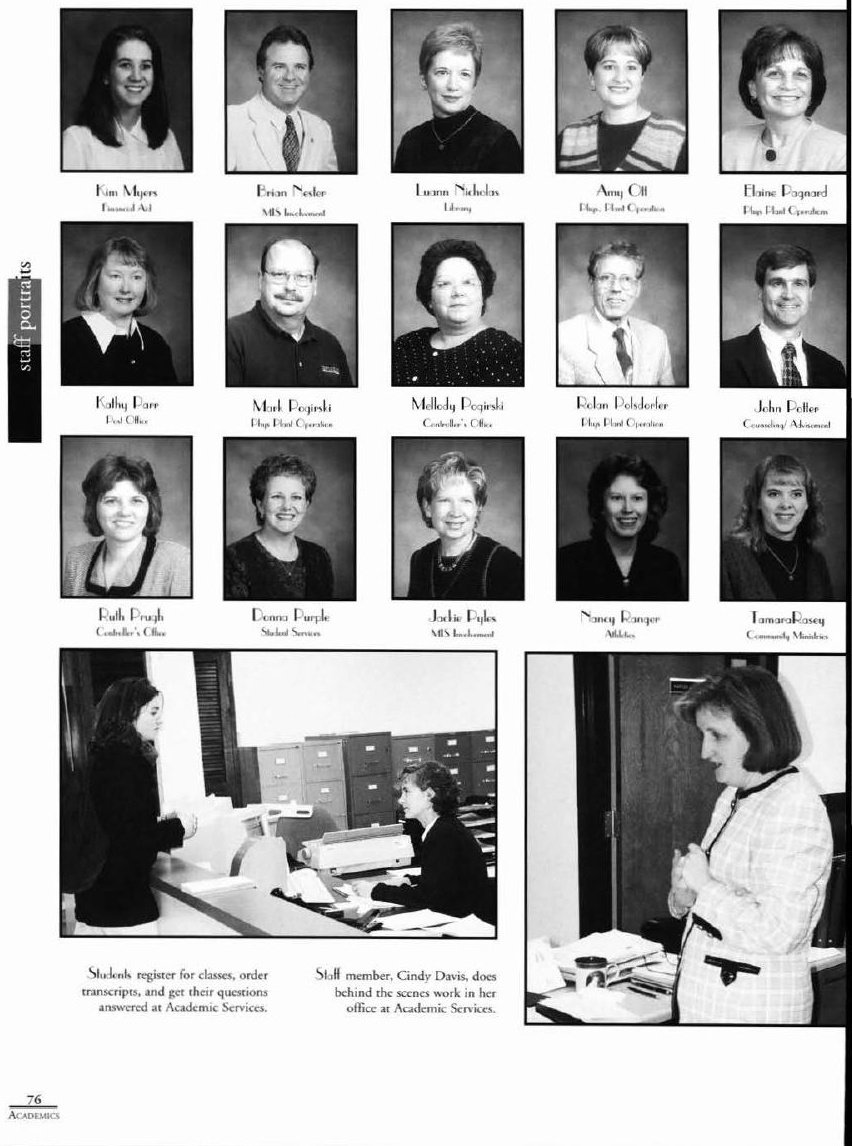




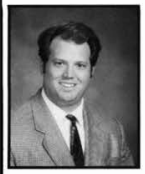

Clinton Rice

$\mathrm{MCChum}$ HI

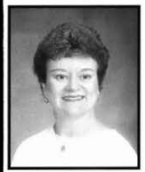

Beverly Rotey

Ditimen Chit

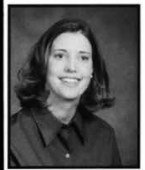

Linsay Sclineider

Acalener Simos

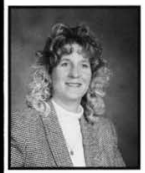

Dawn Scoll

Campas Alodes

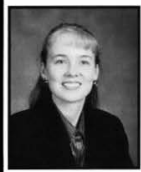

Kara Steinman

Futh Folution

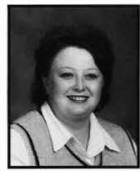

Lyndell Rising Jcheson $\mathrm{BL}$

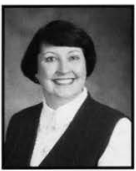

Lyma Rohm

Froulanti Cllin

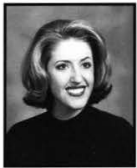

Courtenay Schoalt Atewsiras

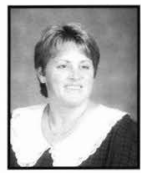

Merilee Shank

Forine \& Spat Seinor

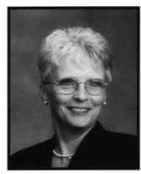

Dolly Stules

Litering

Diredor of Food Services, Chuck McKinney,

has fed Cedarville College students for the

last twenty years. He hopes to serve them for

another twenty years.

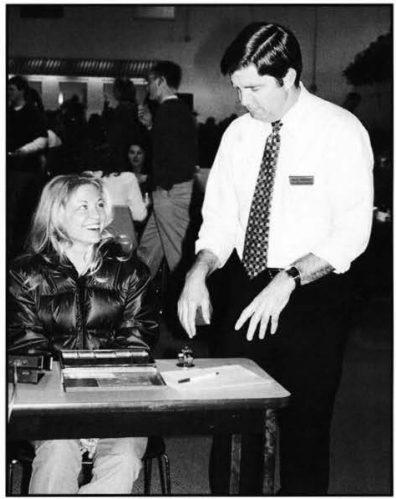

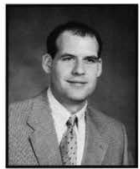

Jensthan Shrubsole

Litrane

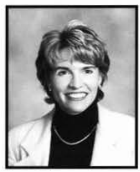

Janice Supplee

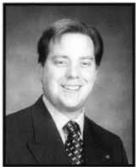

Benjamin Smith

Coabelleristice

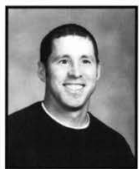

Roger Swiged

Nhlthir F wilition

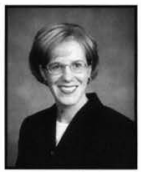

Amy Slart

Inosial AI

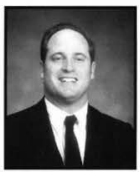

Slawen Thempson fiemed AJ 


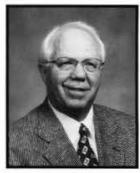

Mar Troyer

OHCCanos Studeals

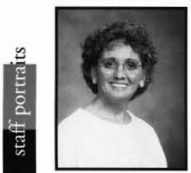

Linda West

Datteren Clines

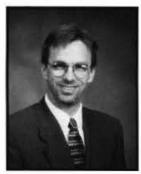

Sleven Winey

Arobere Snows

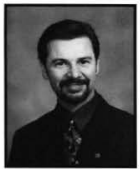

Sluart Zaharek

Alaniminons

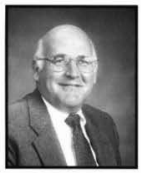

Dick Woller

Campas Achedic

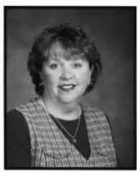

Nellie Wost

Bublater

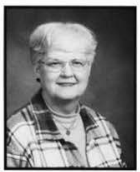

Glenda Wemack

Foul Cilse

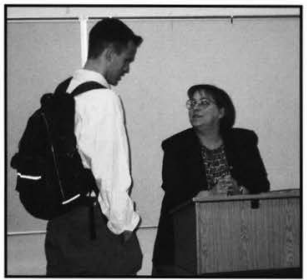

New to the college family, Professor

Warner lends a

listening ear to her student Ben

Thompson.
After thirty years of teaching, Dr. Halsey continues to challenge students to pursue lives of spiritual excellence in today's sociecy.

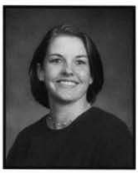

Tricia Walker

Itrany

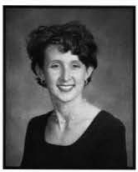

Suman Wat

MA Kimen DD

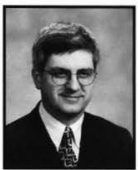

Mart Wemack

Spork lefinualeon

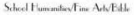

Sharon Woo

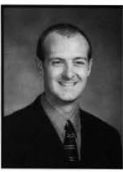

John Wambold

Conemunty, Mination

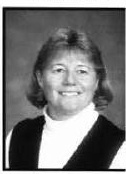

Joy Williams

Dulaece Iriraim
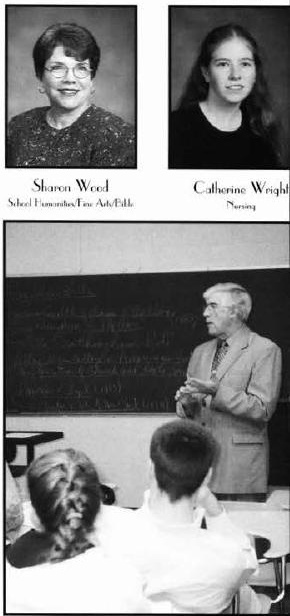

Callerine $W_{\text {riqht }}$ Nerring

(1)




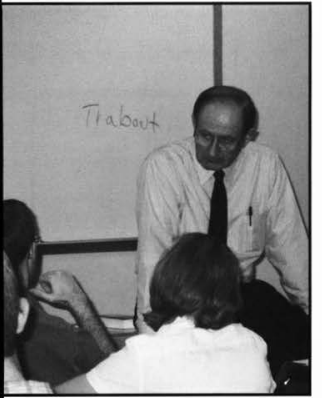

Dedicaled ro his srudenrs, Dr. McGoldrick shares his knowledge of history.
Classmales get together to work at the library.
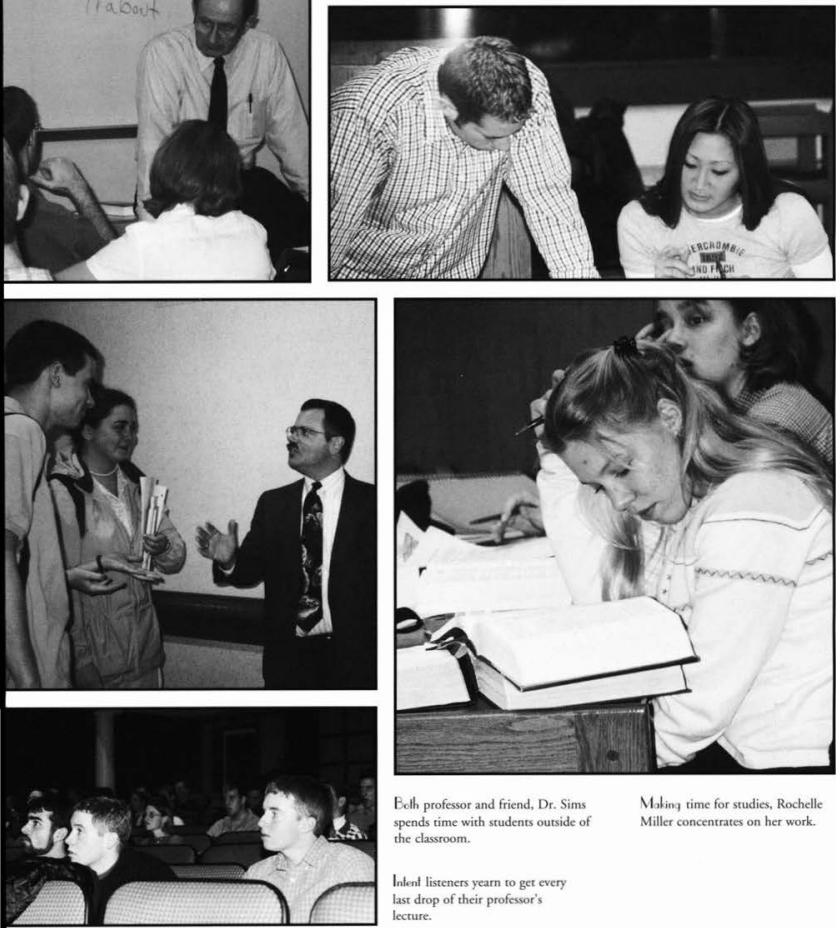

Both professor and friend, Dr. Sims spends time with students outside of the classroom.

Inlent listeners yearn to get every last drop of their professor's lecture.
Making time for studies, Rochelle Miller concentrates on her work. 
This is the verdict: Light has come into the world ... 


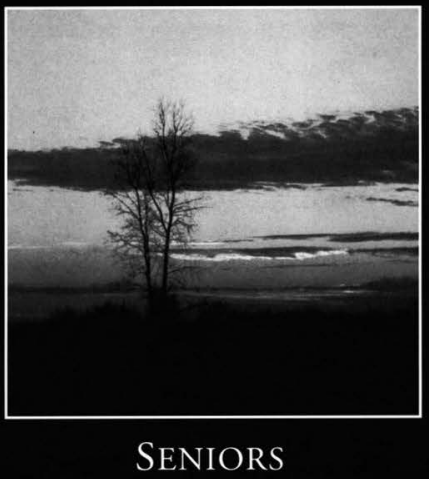




$$
\begin{gathered}
-\mathrm{S}-E-\mathrm{N}-I-\mathrm{O}-\mathrm{R}-\mathrm{S}- \\
\text { class of } \mathbf{2 0 0 0}
\end{gathered}
$$

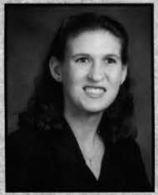

Iennifer D. A66ey Music

Cedarville, $\mathrm{OH}$

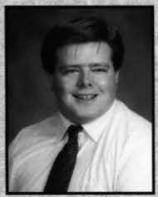

Arik Akerbery

Electrical Enginecring

Cedarville, $\mathrm{OH}$

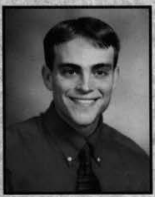

Titus Allen

Pre-Law

Findlay, OH

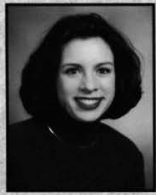

Efisabeth Katherine $9 b c$

Elementary Education

Pittsburgh, PA

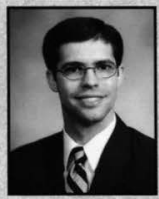

Eric Allcom

Mechanical Enginecring Springfield, MO

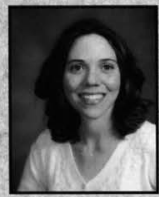

Leafi goy Alley

Elementary Education Hollis Center, Maine

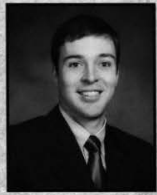

Caleb Acton

Finance/CIS

Pickerington, $\mathrm{OH}$

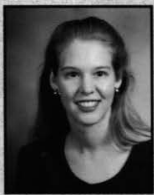

Amy Allen

Music Education

Cumberland, MD

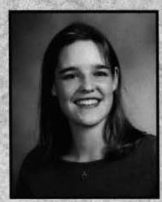

Senrifer Ament

Special Education Murtysville, PA

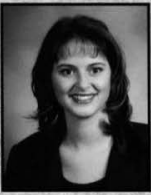

Melissa Dawn Adam Business Comm. Tech. Middletown, $\mathrm{OH}$

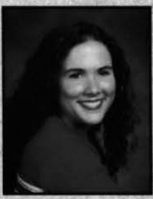

Danette Allen

Elementary Education Cedarville, $\mathrm{OH}$

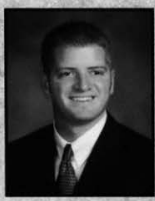

Timothy W. Amstute International Business Union Grove, W1

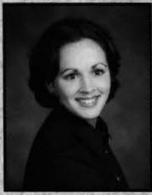

Stephanie Aittama Accounting Zeeland, MI

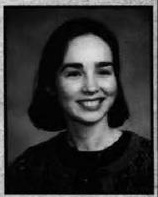

Hannafi g. Allen

Elementary Education Cedarville, $\mathrm{OH}$

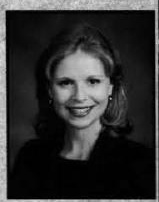

Cara Anderson English Education Uniontown, $\mathrm{OH}$ 


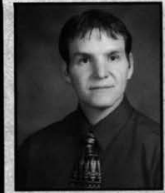

Dana C. Anglund

Biology

Longmont, $\mathrm{CO}$

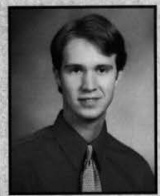

Daniel Avery

CIS

Johnson City, NY

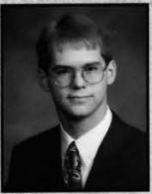

Matthew $T$. Bald tuin

Mechanical Engineering

Danville, IN

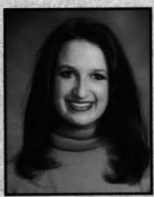

Asfiley M. Barmes

Communication Arts Littleton, $\mathrm{CO}$

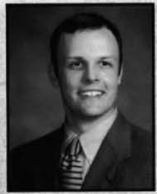

Cfrarles Arcfibald

Athletic Training

Simpsonville, SC

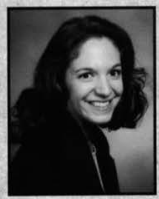

Melfie Bailey

Nursing

Glendora, CA

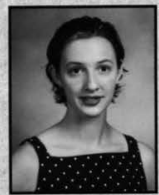

Clarissa C. Band

Comm. Arts/Theatre Arts

Londonderry, NH

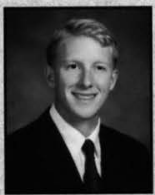

Jeffrey $M$. Barrons

Mechanical Engincering Caro, MI

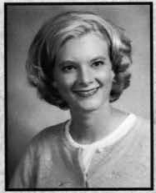

Jenry Amnour

Psychology

Rochester, NY

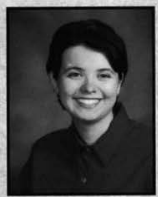

Jamie Luefla Baker

Elemntary/Special Ed.

Addison, NY

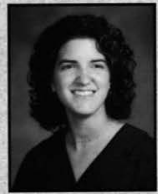

Betfany Gail Barch

Biology

Morris Plains, NJ

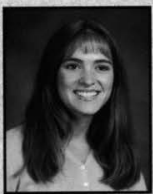

goanna S. Bartiett

Mathematics

Cedarville, $\mathrm{OH}$

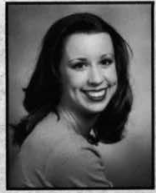

Elizabeth A. Augustus

Nursing

Dayton, $\dot{\mathrm{H}}$

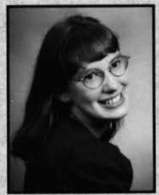

ganefle L. Bafer

Elementary Ed./Spanish

Cardington, $\mathrm{OH}$

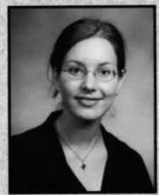

Melanie Barker

Music-Piano Pcdagogy

Ancienne-Lorete, Canada

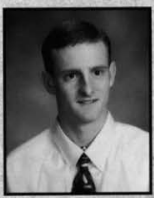

Ron Becter

MIS

Canal Winchester, $\mathrm{OH}$

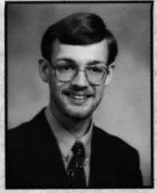

Prifip Ausfafif

MIS

Peoria, IL

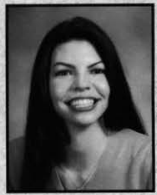

Ranee Marie Baker

Accounting

Lapeer, MI

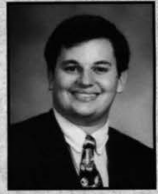

Bradford Barmard

Math and Physics

Springficld, $\mathrm{OH}$

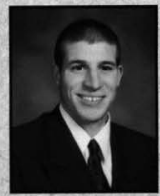

Peter Bedrarek

English Education

Greenfield Center, NY 


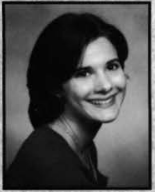

Karen L. Belding

Professional Writing Beckley, WV

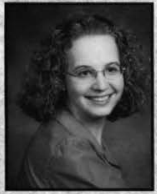

Sandra Bennett

History/S.S./Secondary Ed. Clinton. $\mathrm{OH}$

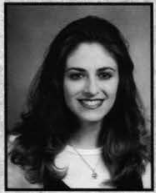

Stacic Renee Bennett Electrical Engineering Cross Lanes. WV

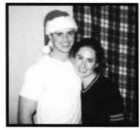

At Christmas time. Jason Olander celebrates with fiance Mary Deweese.

Caren Furst and Joy Hester enjoy doing thcir laundry!

$-\mathrm{G}_{-}-\mathrm{E}-\mathrm{E}-\mathrm{A}-\mathrm{T}-$ $\mathrm{fr}_{i} \mathrm{en}_{\mathrm{d} S}$

Matt Call, Grady Peeler, and Petc Warinsee enjoy Time Square

Carolyn Deltaan, Lindsay Borland, and MandyMcGuire pose during homecoming parade.
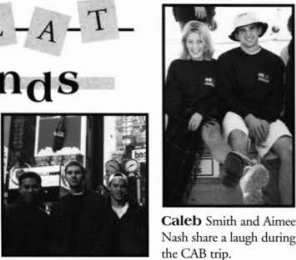

Caleb Smith and Aimee Nash share a laugh during the $C A B$ trip.

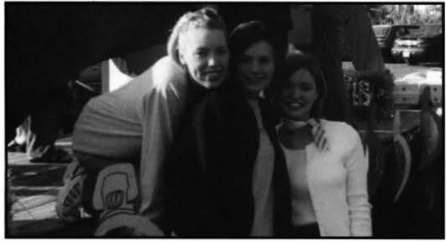

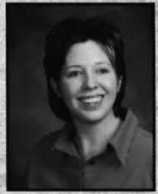

Safrinta Ann Berry

Elementary Education

North Salem, IN

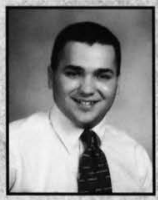

Jim Billock

Nursing

Ravenna, Oh

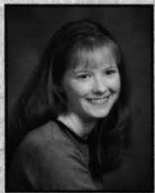

Karen Ann Bjornstad Nursing

Cedarville, $\mathrm{OH}$

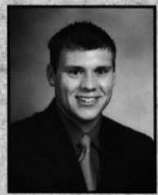

Andrew Blodgett

Biology

Elkhart, IN

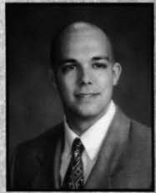

Paul Mndrew Best

Chemistry

Albany. NY

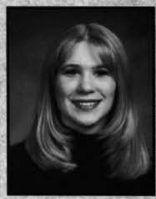

Dycole D. Bills

Org. Communicarions Mio, MI

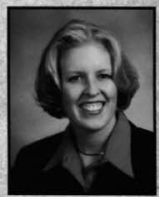

Becca Blackburn

Org. Communications Granger, IN

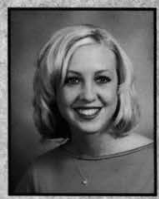

Carissa Anne Bodenmiller Elementary Education Wertfield, NY 


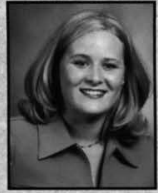

Leafi Vicole Caldwell

Communication Arts

Dayton, OH

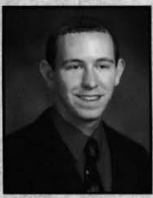

Justin Carboneau

CIS

Saxtons River, VT

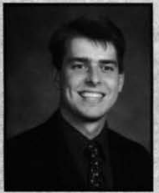

Derck Chandler

Mechanical Engineering

Hastings, MI

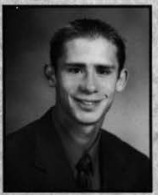

Andrew David Chouinard Athleric Training/Phys. Ed. Raymond. NH

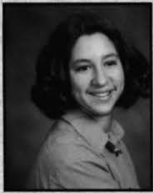

Dayna C. Cafise

Psychology

Milford, PA

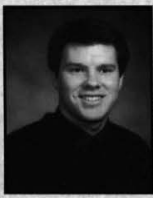

7. David Carr

Nursing

Beavercreek, $\mathrm{OH}$

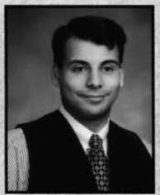

Paul A. Cfievere

Psychology

Guaynabo, PR

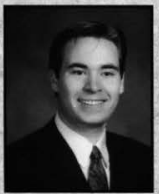

Brenon G. Christofer MIS

Republic, $\mathrm{OH}$

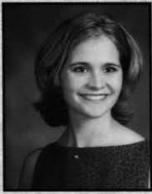

Kirsten Call

Nursing

Dale City, VA

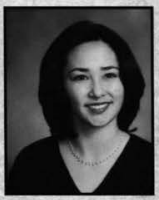

Catherine L. Carter English

Columbia, MD

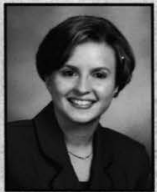

Michelle D. Chiarelli

Early Childhood Ed.

Camey's Point, NJ

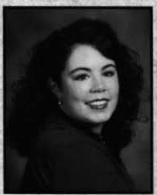

Carla clark.

Bible Comp/CE Youth Cedarville, $\mathrm{OH}$

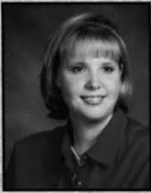

Alison L. Camillo

Business Management Salem, OR

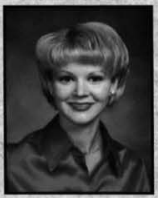

Cara C. Carver

Applied Psychology

Valparaiso, IN

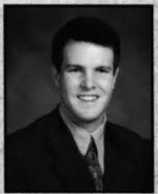

Natfian R. Cfillfers

Christian Education/Youth Huntington, WV

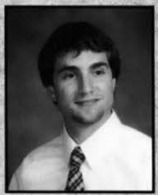

Mark Clark

Mehcanical Engincering Grecnville, $\mathrm{OH}$

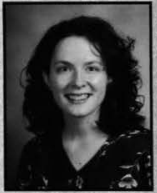

Robyn R Cainon

Music-Piano Pedagogy Springficld, MO

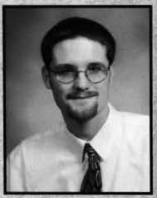

Brandon Caudill

Biology

Goshen, IN

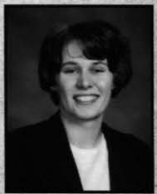

Emuna Jolie Chmura

Bible Comprehensive

Columbia Station, $\mathrm{OH}$

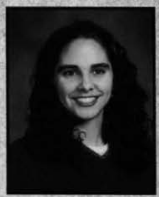

Valerie Kaye Claus

Psychology

Tiffin, $\mathrm{OH}$ 


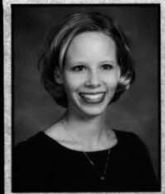

Jennifer Co6b

User Information Systems

Sissonville, WV

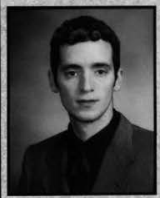

Mark Collins

Biology

Lutz, FL

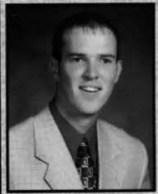

Tracy Wiffiam Commons Bible Education

Harrisburg, PA

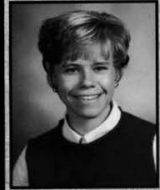

Lucinda Congdon

Business Comm. Tech.

Randolph, NY

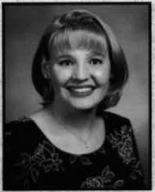

genuifer Cobb

Nursing

London, $\mathrm{OH}$

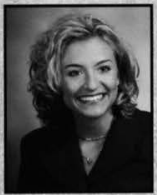

Amy Lea Combs

Applicd Psychology

Cedarville, $\mathrm{OH}$

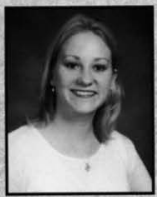

Cfuistine Micielle Compton

English Education

Waterford, MI

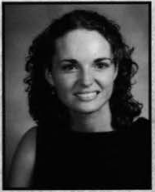

Jenny Susanne Coble

Earty Childhood Ed.

Burlington, NC

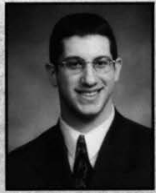

Ryan S. Cofian

Christian Education/Youth Salem, CT

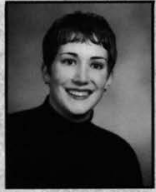

Charity cole

Physical Education

Sherman, NY

$$
\begin{gathered}
-C-E-D-A-R- \\
\mathbf{l a k e}
\end{gathered}
$$

Who could ever forget Cedar Lake and their hundreds of walks past, around, and over it throughout their years at Cedarville? From a distance, the lake appeared tranquil, picturesque, and even beautiful. Throughout the long winter months, the frozen and often snow-covered lake was a reminder of God's beautiful creation. It also served as a recreation field for some stu-

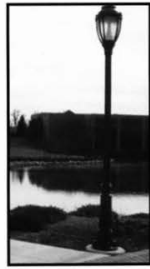
dents, despite Campus Safety's public warnings against ice walking. As the weather wamed, the lake's aroma and surface were often tainted by growingalgae. However, the colorful eveningsunsets still reminded students why Cedar Lake was such a memorable part of campus.

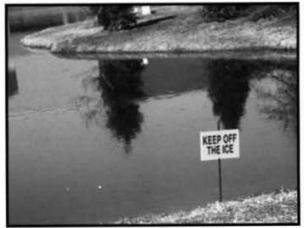

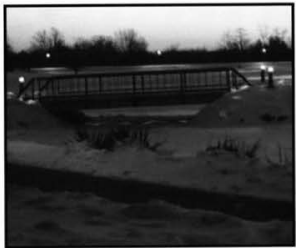

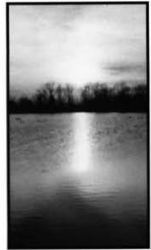




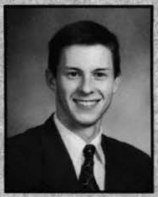

Shawn R. Conley

Criminal Justice

Whire Oak, PA

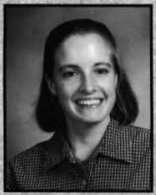

Angela M. Cooley

Bible Comp/CE Yourh

Cedarville, $\mathrm{OH}$

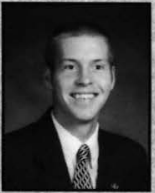

Jeremy Couture

Pre-Seminary

Sturgis, MI

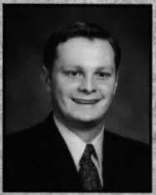

Rlex Crumt

Mulri. Tech./Broadeasting

Tracys Landing, MD

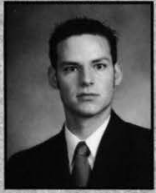

David Cook

Broadcasting

Sewell, N]

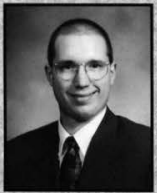

Brett C. Cooley

Bible Comp/Zastoeal Studies

Cedarville, $\mathrm{OH}$

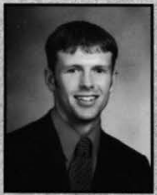

gason covilf

Mechanical Engineering

Nottingham, NH

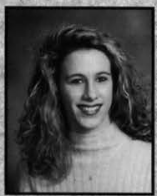

Amy S. Crunelle

Finance

Columbus, $\mathrm{OH}$

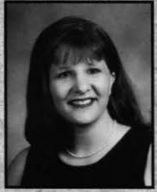

Jennifer C. Cook

Music Education

Perrysburg, $\mathrm{OH}$

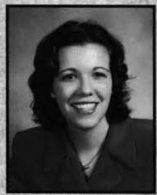

Tina M. Coon

Biology

Kalamazoo, MI

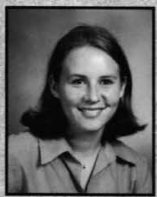

Andrea D. Creamer

English Education

Xenia, $\mathrm{OH}$

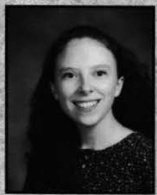

Brita Culberson

Elementary Education

Cedarville, $\mathrm{OH}$

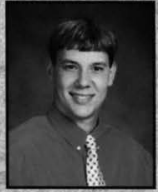

Mattifew Cook

Electrical Engincering

Cedarvills, $\mathrm{OH}$

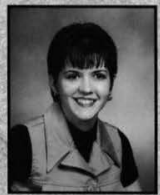

Tiffiny Marie Cope

Nursing

East Sparra, OH

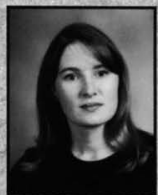

Suzanna Creps

Biology

Modesto, CA

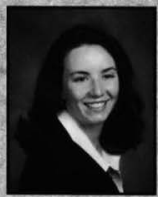

Clinista Marie Culler

Communication Arts

Garden Ciry, MI

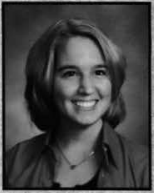

Susanna 9. Cook

Elementary Education

Cedarville, $\mathrm{OH}$

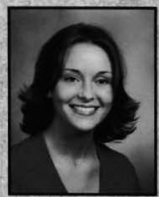

Amber Dazun Corbin

Applied Psychology

Fairland, IN

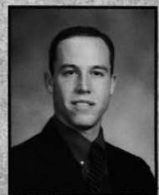

Dustin W. Crider

Accounting/Finance

Pioneer. $\mathrm{OH}$

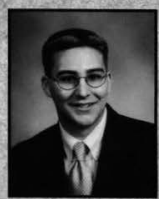

yordan culp

Mathematios

Downingrown, PA 


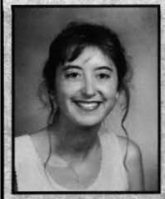

Dara Rfiel Curningfiam English Education/TESI

Parkersburg, WV

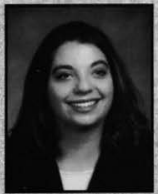

Amber Dafuns

Org. Communications Findlay, $\mathrm{OH}$

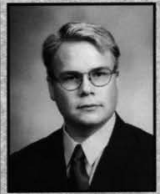

Jofin Dearie

Sports Management Lakeland, Fi.

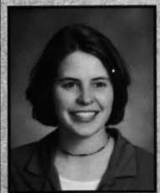

Jalene Dillon

Elementary Education Longmont, $\mathrm{CO}$

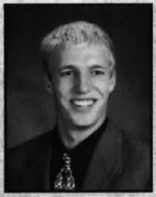

Jonatfian Cunuingfiam CIS

Cedarville, $\mathrm{OH}$

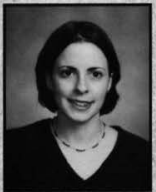

Catfiy L. Dato

English

Derry, NH

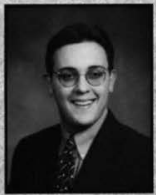

James Deaton

Professional Writing/Biblc Hillsboro, $\mathrm{OH}$

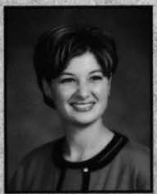

Kena Doden

Philosophy

Aubum, IN

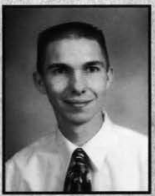

Shaum Dabravalskas History

Grove Ciry, OH

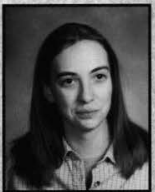

Lia R Davenport

Office Systems Management Essexyille, MI

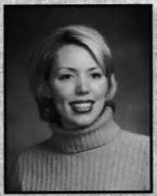

Carolyn De Haan

Nursing

Grand Rapids, MI

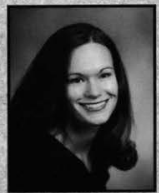

Candace Dodge

Nursing

Clear Spring, MD

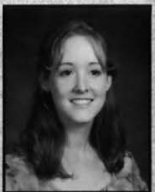

Tracy Dabravalskes

Office Systems

Sewickley, PA

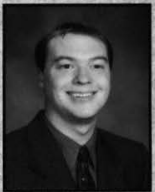

Cory Davids

Marketing

Clive; IA

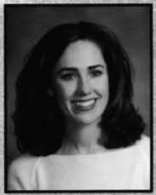

Mary Kristin Deweese

Elementary Education

Eairfax, VA

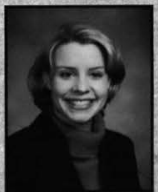

Jentifer D. Dolby

Spanish Education

Cincinnati, $\mathrm{OH}$

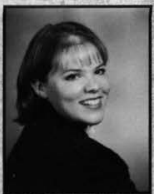

Hannafi $R$. Dager

Early Childhood/Elem. Ed. Shamong, NJ

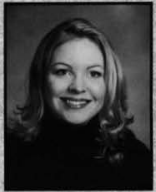

Cfristina Dean

Finance

Rochester, NY

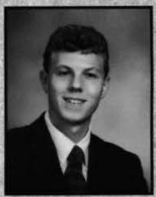

Iolin Diggle

Electrical Engineering

Oswego. IL

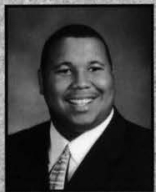

Michael $\mathcal{L}$. Dorsey

Org Comm.Theater Baltimore, MD 


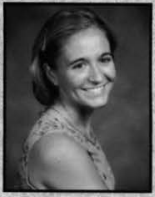

Dawn Driesbact

Elementary Education St. Charles, II

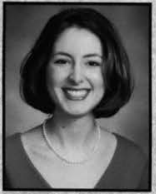

Melanie Esther Edwards

Applied Psychology

Williamsville, NY

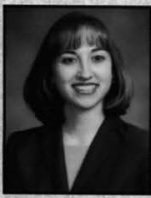

Karyn Farkus

Music Education

Temple, TX

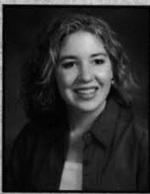

Jufie A. Finnigan

Mulrimedia Technologies

Penn Yan, NY

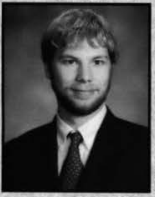

Danief C. Dye

InternationalStudies Cable, $\mathrm{OH}$

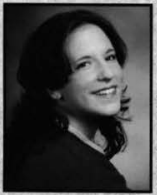

Micfielle Edwands

Biology

Jackson, MI

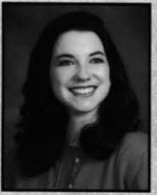

Andrea Farlow

Communications

Cedarville, $\mathrm{OH}$

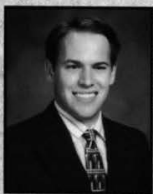

Bill Fisciter

Electrical Engineering

Northridge, CA

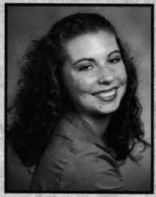

Adriante L. Eads

English Education

Jackson, MI

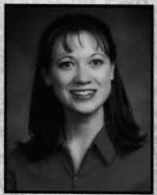

Sama joy Elevelif

Church Music

Mattawan, MI

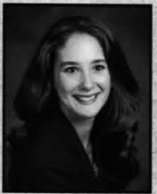

Iufia Farlow

Finance

Cedarville, $\mathrm{OH}$

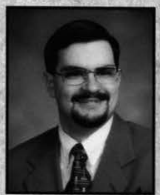

Matt A. Fiare

Org. Communications

Xenia, $\mathrm{OH}$

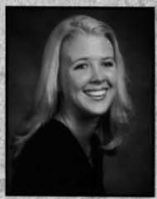

Karen Eccleston

Athletic Training

Titusville, FL

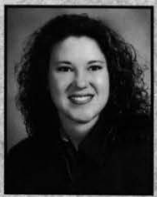

Andrea Kay Endicott

Org. Communicarions

Liberty Center, OH

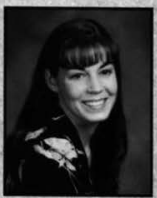

Jennifer Joy Ferguson

Elementary Educacion

Baltimore, MD

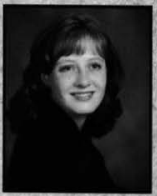

Sarafi Elfen Flenar

Professional Writing

Columbia Ciry, IN

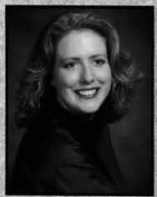

Amy 0. Edwands

Psychology

Gahanna, $\mathrm{OH}$

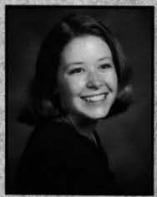

Carrie Fabian

Elementary Education

Burt, MI

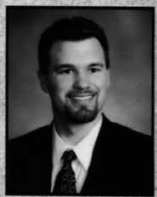

gosfina R. Fetzer Marketing

Indianapolis, IN

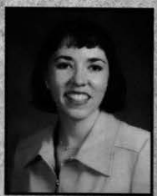

Denelda Afison Folte

Church Music/Business

Northfield, $\mathrm{OH}$ 
Gabe Schlappi and Scott Walkergoof off.

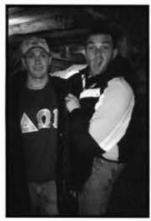

$$
\frac{-F-U-N-N-Y-}{\text { times }}
$$

Megan Wawro

breaks for lunch with a friend, while student reaching in Jerusalem

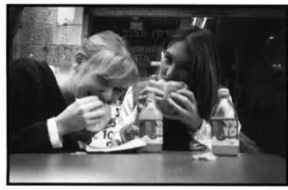

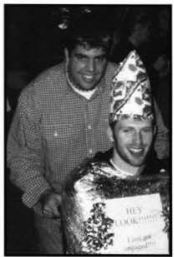

Jason Covill gets the royal treatment after popping the question.
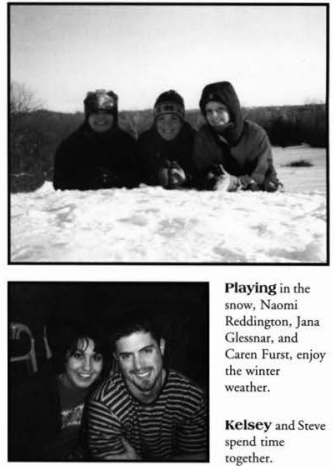

Playing in the snow, Naomi Reddington, Jana Glessnar, and Caren Furst, enjoy the winter weather.

Kelsey and Steve spend time together.

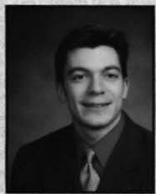

Josfua M. Fronduti

Charch Music Ministry

Forest City, PA

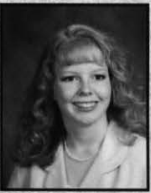

Caren E. Furst

Nursing

Appleton, WI

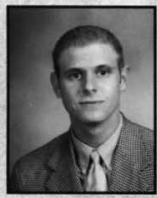

Thomas C. Iry

Christian Ed/Yourh

South Vienna, $\mathrm{OH}$

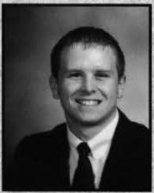

Kyle E. Gantz

Biology

DeGraff, $\mathrm{OH}$

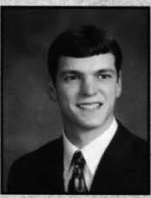

Ionathan D. Fuge BSME

Landisville, PA

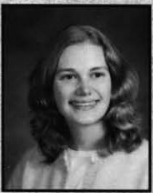

Melinda Gates

Nursing

North Muskegon, MI

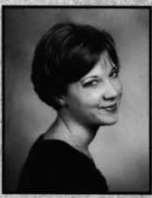

Doreen Erifa Futtr

Bible/Elementary Ed

Blairstown, NJ

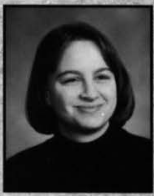

Christina Gideon

Professional Writing

Sergeant Bluff. IA

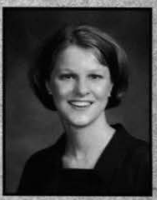

Chistina Janifer Fullerton

Elementary Education Germantown, IN

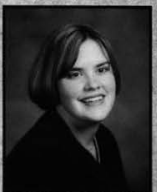

Sarah Gilefirist

Mathernatics

Clarks Summir, PA 


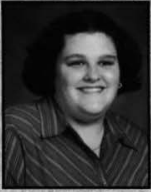

Elizabetit R. Grosztyla

Elementary Education

Londonderry, $\mathrm{NH}$

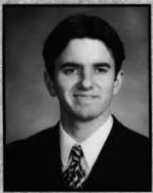

Yames Bisfiop Hall

Communication Arts

FaiffaxStation, VA

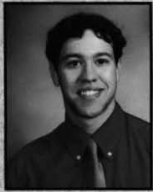

Micfiael R Hauser

Multimedia Tech

Jersey Shore, PA

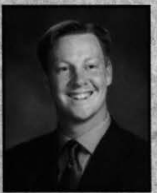

Andrew 9. Hedges

Com, Arrs/Christian Ed

Massillon, $\mathrm{OH}$

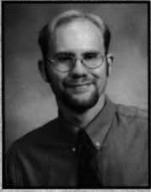

Stepfien R, Guentfiter Ir. Broadcasting Smithsboro.NY

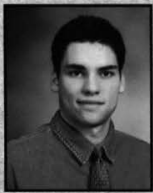

Jonatfian A. Hallsten Electrical Enginecring

Hoffman Estates, II.

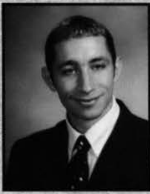

Mark A. Hayner

Broadcasting

Penn Yan, NY

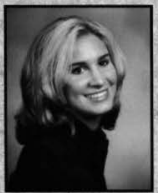

Robyn Cheree Feffner English/Prof.Writing Lima, $\mathrm{OH}$

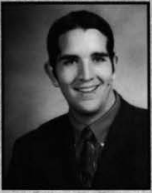

Paul R Gwilt

Pre-Law/History

Omaha,NE

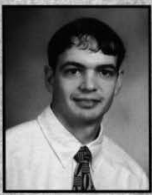

Micfiael Anthomy Hammond

Elementary Education

West Manfield, $\mathrm{OH}$

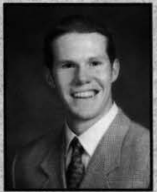

James C. Feadings

MochanicalEngincering

DeGraff, OH

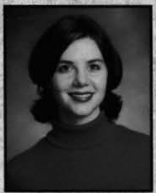

Christina Rene Heftin

Org. Communications

Gresham, OR

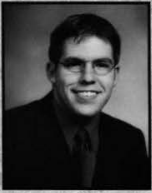

Daniel S. Facker

Marketing/Management

Columbus, $\mathrm{OH}$

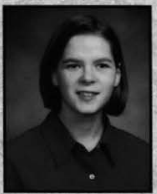

giffianne C. Hancock

Elementary Education

Springboro, $\mathrm{OH}$

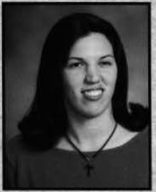

Leanne Priscilla Heath

Special/Elementary Ed.

Ravenna, $\mathrm{OH}$

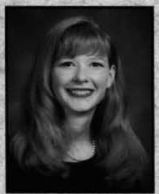

Christine Henderson

Special/Elementary Fd.

Cedarville, $\mathrm{OH}$

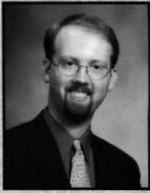

Kevin David Haisch

Electrical Engineering Greenville, NC

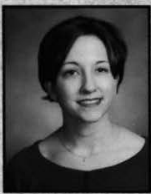

RoseAnn G. Hassell

Accounting

New London, WI

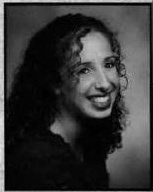

Molly Ruthi Featon

Special/Elementary Ed.

Dayton, $\mathrm{OH}$

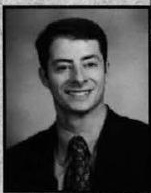

Chiris W. Herman

Management Lake Forest, II 


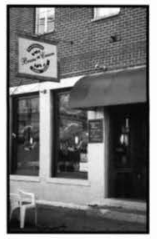

Beans n Cream is a hot spot for students.

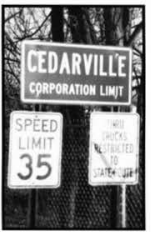

This green sign defines Cedarville's area limits.

Clifton Mill provides beautifal scenery for students.

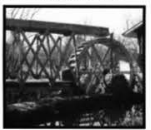

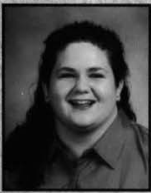

Heatfier Marie gacfson English Education Fort Wayne, IN
Mom and Dad's is the McKinney and

best place for penny candy McChesney scrve as home and milkshakes. for many seniors this year.

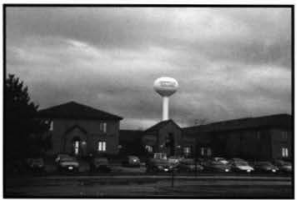

- N-E-A-R.B-Y landmarks

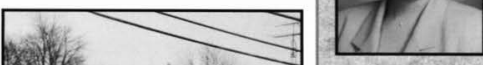

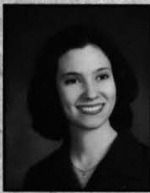

Eried Susan Jenfints Elementary Education Centerville, $\mathrm{OH}$

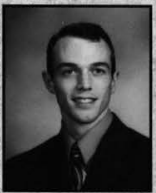

Nathan I. Jenkins Mechanical Engineering BallstonSpa, NY
Karen Ann Huber Spanish

Rockton, IL.

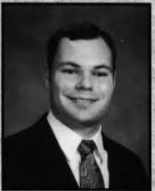

James Steven Fulbert Mechanical Engineering Rockford, MI

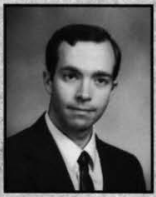

Royce Hunt

History

London, $\mathrm{OH}$

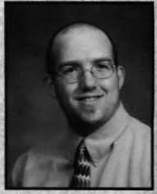

Casey Luke Huebrer History

Milwaukee, WI

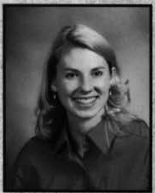

Pamela Joy Huls

Elementary Education Fort Wayne; IN

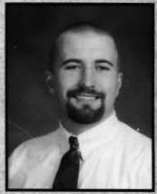

Jeremy Dean Furley

Criminal Justice

Belmont, MI

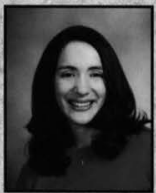

Grace Marie Jofransen Elementary Education Cedarville, $\mathrm{OH}$

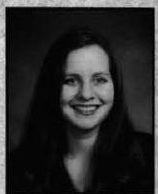

Efizabeth Anne Jofinson Management Waterloo, IA 


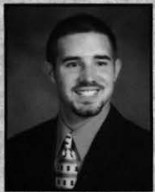

Midial Kostuck

Bible Comprehensive

Brookficld, IL

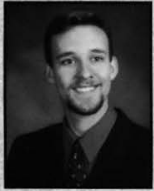

Daniel L. Kulast

Electical Engineering

Grand Rapids, MI

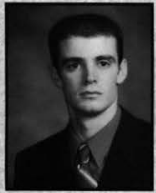

Michael David Lampton

Physical Education

Jeffersonville, $\mathrm{OH}$

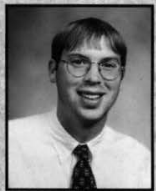

Iason Andrew Lee

Psychology

Cedarville, $\mathrm{OH}$

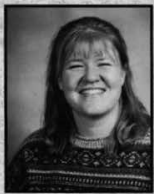

Vianna Kreuder-Crowell Elementary Education

Cedarville, $\mathrm{OH}$

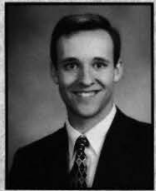

Timotfy Richand Kwast

Electrical Engineering

Grand Rapids, MI

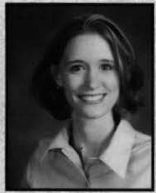

Cfuristine D. Lancaster

English Education

Rawlings, MD

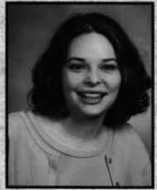

Brandi Leeds

English

Quincy, II.

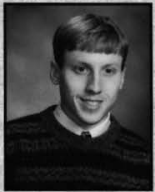

Andrew Jonatfian Krum

Mathematics

Catawissa, PA

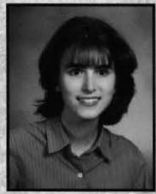

Ellen Laber

Bible Comp./Gen. Studies

Newport, VT

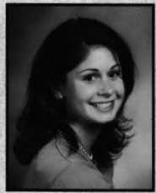

Rebecca Leatherman

Elementary/SpecialEd.

Harleysville, PA

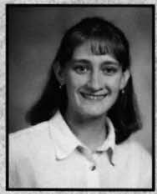

Suzamie Lymn Lefiman

Exercise Science

Orrville, $\mathrm{OH}$

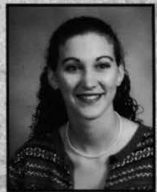

Kristin 9. Kufin

Elementary Education

Lebanon. $\mathrm{OH}$

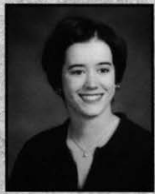

Hannafi Marie Lafes

Finance

Buda, TX

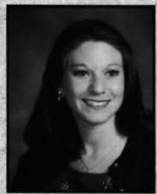

Dequn Michelle Lee

Elementary Education

Cedarville, $\mathrm{OH}$

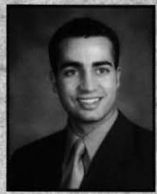

Scott Micfiael Lefir

Preseminary

Flint, MI

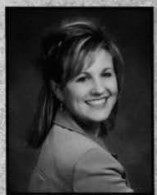

Barbi Joy Xuzsfinifov

Elementary Education

Erie, PA

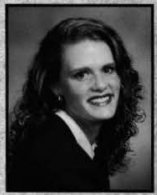

Iufie $X$ Lamborn

Nursing

Parkesburg, PA

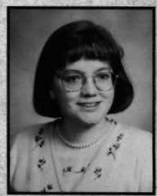

Heather Lymn Lee

Elementary Education

Cedarville, $\mathrm{OH}$

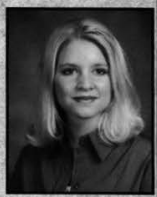

Shanna Ann Lerfiart Nursing

Grand Blanc, MI 


$$
\begin{aligned}
& -\mathrm{S} \cdot{ }^{E}-\mathrm{N}-{ }^{I}-\mathrm{O}_{-} R- \\
& \text { moments }
\end{aligned}
$$

\section{moments}

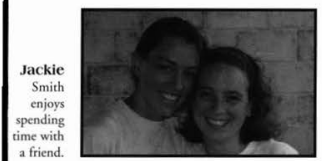

Harriman girls

celebrate Christmas.

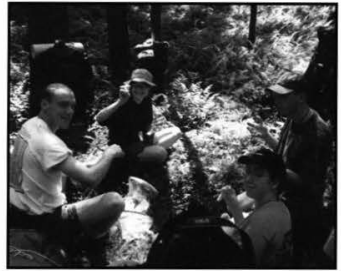

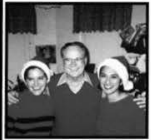

Nikki Thomas and Rachel Puckett pose with Dr. Dixon

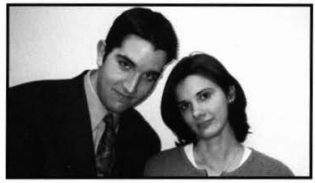

Nathan Lewis and friends hit the campground.

Dressing as southern belles is always fun!

Paul Gwilt and Karen Belding attempt to look serious.

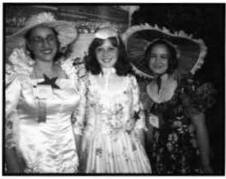

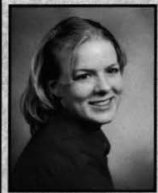

Kimberíy B. Leverson Nursing

Highlands Ranch, $\mathrm{CO}$

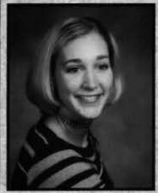

Jemifer $M$. Linton

Business Management Lexington, $\mathrm{OH}$

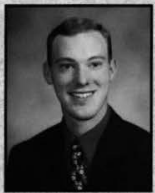

Nathan A. Lewis

Mechanical Engineering Salem, OR

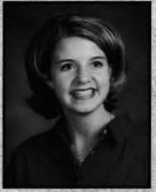

Katie M. Little

Nursing

Ballston Lake, NY

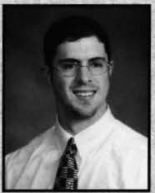

Stepfien Jonatfian Ligfit Chemistry

Wrentham, MA

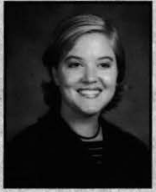

Lindsey Ann Lorinovich Political Science Montpelier, VT

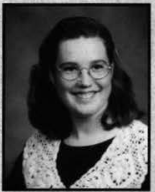

Tiffany Ann Linden

Elementary Education Sun City, AZ

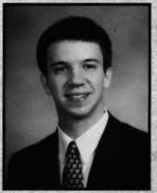

Micfiael R Luck MIS

Fort Washington, MD

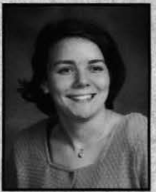

Betsy Emefia Linnell

Special Education

Montpelier, $\mathrm{OH}$

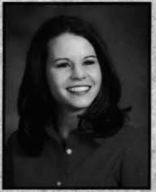

Dicole \%. Lucformann Communication Arts Naperville, IL 


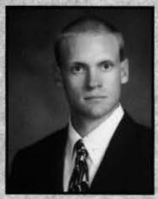

Brandon W. Luke

Mechanical Enginecring

Kearney, NE

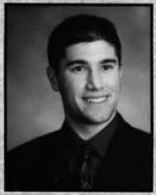

Gason Daniel Manciesfy

Finance

Muskegon, MI

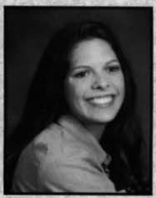

Rosemarie Marotta

Elementary Special Ed.

Lockport. NY

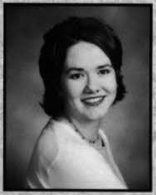

Saraft Efizabcti Mayer

Elementary Educacion Anoka, MN

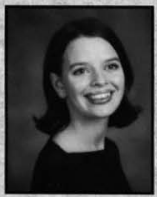

Jessica Joy Lutz

Org. Communications

Orlando, FL

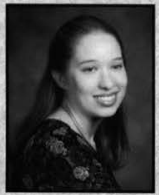

Kimberly R. Mapes

Secondary Education

Rochester, NY

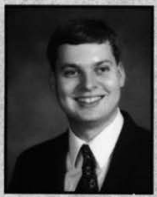

Jolin Mark Mason MIS

Cedarville, $\mathrm{OH}$

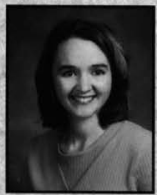

Molly Meclure

Elementary Education

Sterling, II

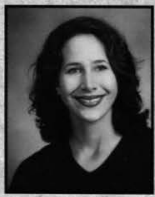

Diana Margaret Lyons

English Education

Clinton Twp., MI

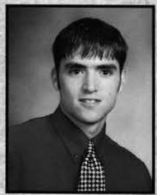

Aaron Margene

History

Birmingham, AL.

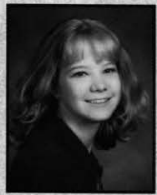

Iilfian Marie Massey.

Accounting

Salem, NJ

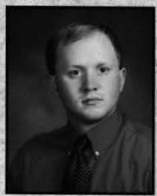

Benjantin K. McComas History/ Political Science Marysville, $\mathrm{OH}$

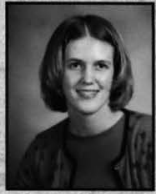

Lisa Eileen MacPherson

Elementary Education

Ghent, NY

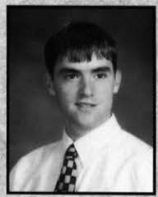

Jereny Margene

Hisrory

Birmingham, AL

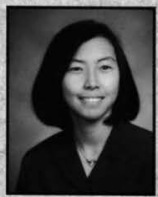

Angela 2 Mast

Elementary Education

Tunkhannock, PA

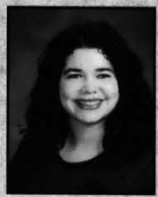

Tamara Dayfe Mccomb Music Performance Evesham, NJ

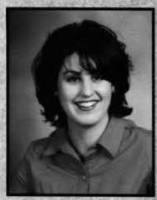

Kelsey M. Mafil

- Elementary Education Anderson. IN

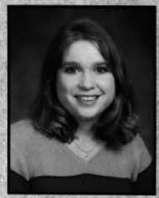

Carol Louise Marks

Business Communications Litir, PA

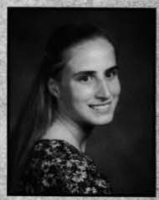

Heatfier Marie Maybiry Electrical Engineering

North Syracuse, NY

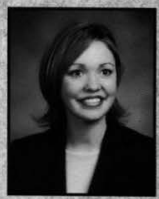

Amanda Claire MeGuire

Communication Arts Dayzon, OH 


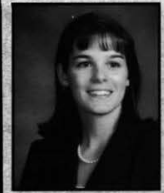

Heidf Christine MCKelvey

Applied Psychology

Pioneer, $\mathrm{OH}$

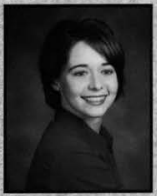

Amanda Beth Melcher

Social Work

Reading, PA

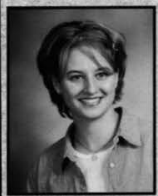

Elizabeth Anne Mills

Nursing

Reynoldsburg, $\mathrm{OH}$

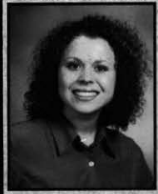

Efisabetfi A. Morris

Elementary Education

Clinton, $\mathrm{Oh}$

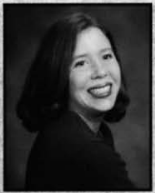

Amanda E. Mclaugfifin

Elementary Education

Galion, $\mathrm{OH}$

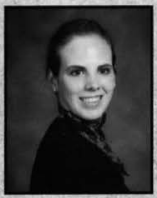

Rachel Eilene Menz

Accounting

Rockbridge, $\mathrm{OH}$

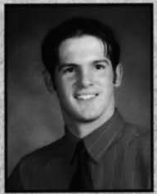

Stepfien Sorman Mofir Spanish

Shelbyville, IN

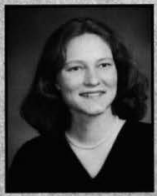

Rachiel M. Morris

Tech./ Professional Writing Saginaw, MI

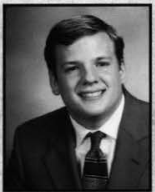

Jorl David McLeod

Bible Comprehensive

Harrisburg. PA

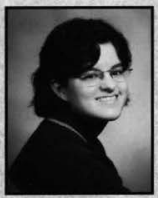

Brenda S. Mick

Nursing

Grand Rapids, MI

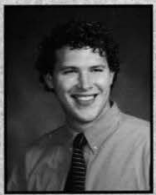

Robert Michael Moll

English/CIS

Schaumburg, IL

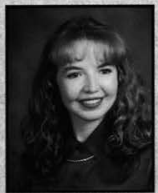

Anty L. Morse

Mechanical Engineering Davidson, MI

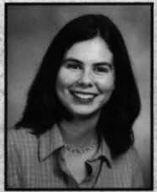

Amy Renee Mead

Nursing

Faribaulk, MN

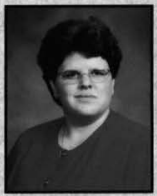

Andrea Beth Mifler

Elementary Education

Monroeville, $\mathrm{OH}$

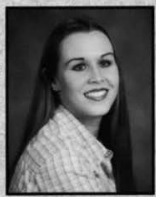

Elizabetfi Susan Moore

Marketing/Management

Allegany, NY

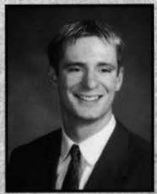

Tobin R. Most

Mechanical Engineering Lansing, MI

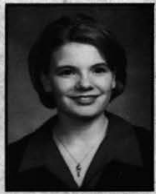

Jessica L. Meissnet

Elementary Education Hartland. WI

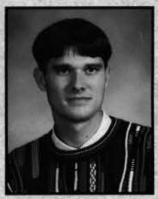

David Robert Mills Biology

Adera, Togo, West Africa

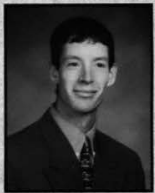

Micfiael Elfiot Moran

PE/ Comprehensive

Plymouth, MI

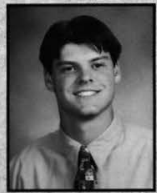

David L. SMowrey

Criminal Justice

Daleville, $\mathbb{I N}$ 


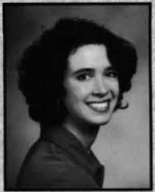

Jeanne C. Moynifian

Biology

Waterford, CI

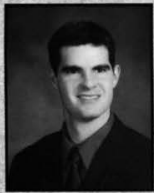

Michael James Munro Nursing

Xenia, $\mathrm{OH}$

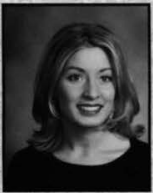

Ainzee B. Nash

Nursing

Grand Rapids, MI

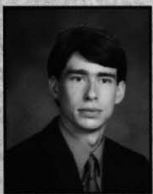

Andrew W. Nuveldt

Music Education

Waterford, CT

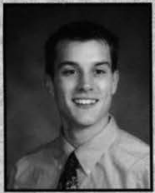

Kyle Aaron Mraz

Marketing/ Management Akron, $\mathrm{OH}$

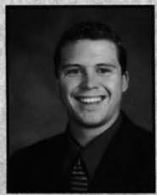

James Wilfiam Murifock. Mechanical Enginecring Brighton, MI

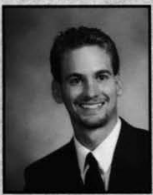

Brad R. Neese

Christian Education

Osceola, IN

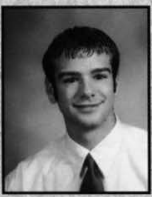

Jason Paul O'veal

Marketing

Simpsonville, SC

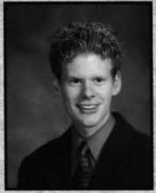

Lncas Keiff. Mudrey

Broadcasting

Omond Beach. FL

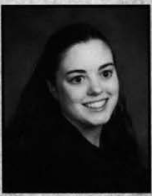

Any Michelle Murphy

Mechanical Engincering

Cedarville, $\mathrm{OH}$

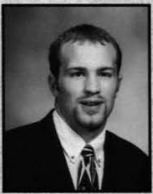

Daniel'E Nichols

Mechanical Engineering Addison, NY

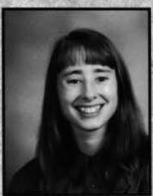

Melissa Joy Odens

Music Composition

Brooklyn Park, MN

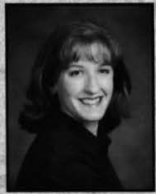

Amanda Lynn Mullen

Spanish Education

Lexington, $\mathrm{OH}$

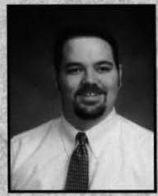

Jason Myers

Philosophy

Belton, TX

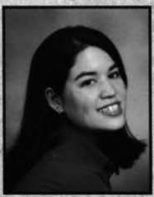

Keifo Lynn Noble

Mathematics

Tunkhannock, PA

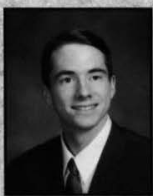

Kurtis Fon Oet Kem

Mechanical Engineering

St. Marys, PA

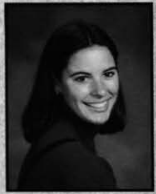

Katie Aun Mummat

Athletic Training

Mechanicsburg, PA

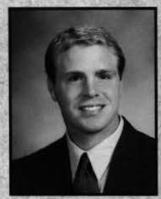

Jofin Michael Myers

Bible Comprehensive

Cedarville, $\mathrm{OH}$

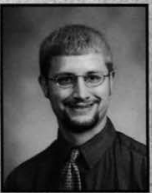

Isaiah Noftz

Broadcasting

Fremont, $\mathrm{OH}$

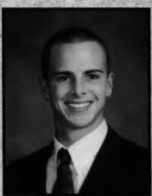

Jason Bert Olander

Accounting

Milwaukee, WI 


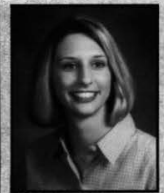

Cfuristine Lym Olbrich Education

Monroeville, NJ

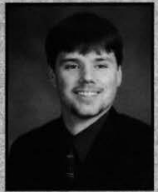

Geoffrey Paul Parlett Comm. Arrs/Broadcasting King Ferry, NY

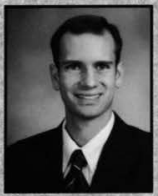

Jordan Scott Patrick Christian Education Lansing, MI

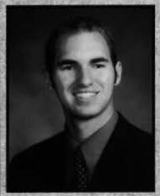

Grady Lee Peefer III Accounting

Barrington, N]

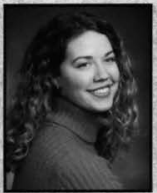

Gudrun Marit OSson Professional Writing. Heron Lake, MN

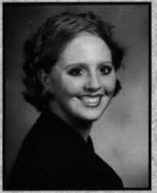

Kristina L. Part Accounting/Finance St. Johns, MI

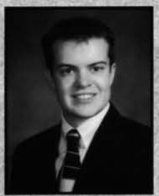

Mattiew E. Pedersen Psychology

Des Moines, IA

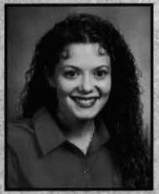

gessien C. Penningtort Nursing

Palmer, AK

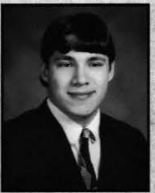

Ieffrey Yonatfian Olson Mechanical Engineering. Union Gorve, WI

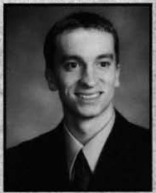
Professional Writing Beaverereek, $\mathrm{OH}$
Iason Paul Oterturf

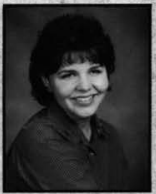

Rachelle Lymn Paquin

Psychology

Yarmouth, ME
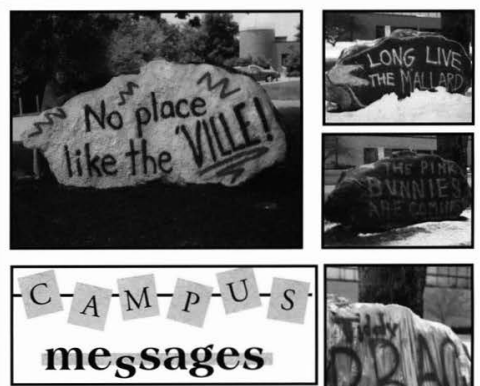

The Cedarville rock will be a special memory of Cedarville. On a regular basis, students crossed campus after dark in order to paint the rock. Serving as the largest source of campus news, the rock notified Cedarville students of birthdays, engagements, spring break plans, and of course, the countdown to graduation. Despite the fact that students did not hang out at the rock as publicized in Cedarville campaigns, the rock cer. tainly held a very special place in the hearts of $\mathrm{Ce}$ darville students as they viewed its mes

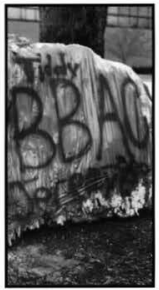
sages daily.

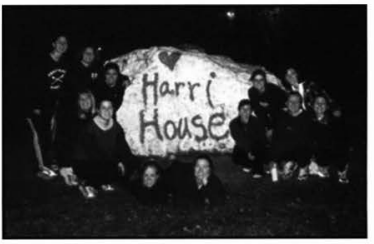




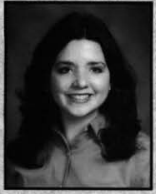

Miriam Efizabetf Perez Biology

Yorktown. VA

Class Song: "You Have Been Good" by Scott Krippayne

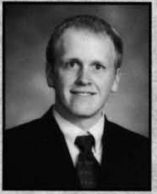

Brandon Douglas Perkins Mechanical Engincering East Chatham, NY

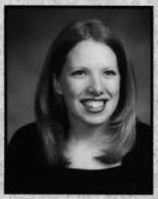

Lindsay V. Peterson

Communications

Largo, FL

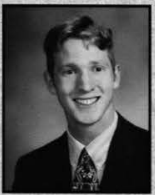

Jofin Michael Perkins

CIS/ Management

Ithaca, NY

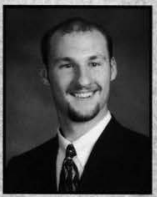

Tunotify Ray Pfafiter Mathematics Education Bucyrus, $\mathrm{OH}$

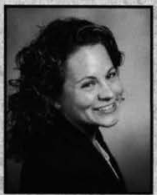

Kelsey Sizanne Perkins Communicarions Aurora, CO

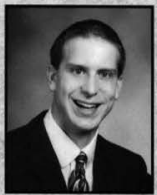

Micfiael Scott Pierson Elementary/Special Ed. Hammond, IN

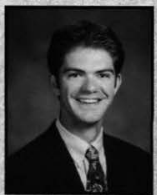

David Paul Perlow)

Technical Communication Union, $\mathrm{OH}$

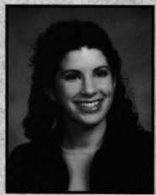

Amy Carol Pitstick Elementary Education Xenia, $\mathrm{OH}$

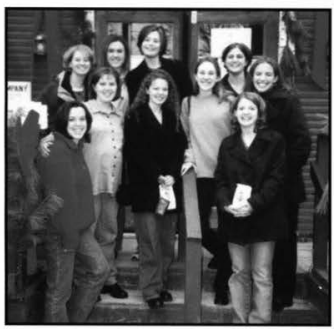

$-F-R-I-E-N-D-S$

\section{forever}

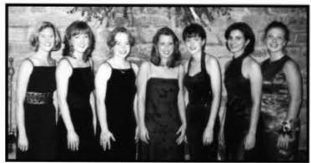

Senior friends attend their last Homecoming Banquet.

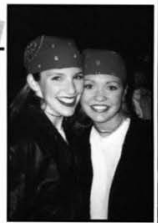

Lindsay Wagner and Mandy McGuire dress up for the Org Fair.

Pete Warinsce and Teresa Tallarita enjoy Tenn.
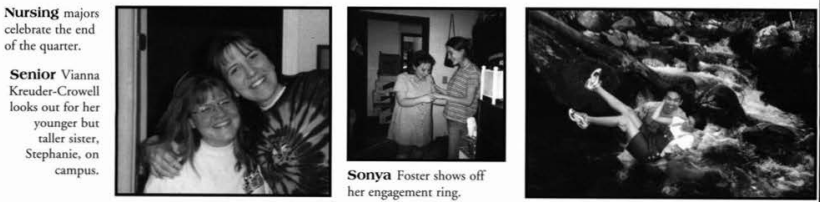

Sonya Foster shows off her engagement ring. 


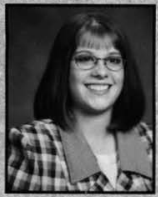

Noomi Redington.

Nursing

Minot, ND

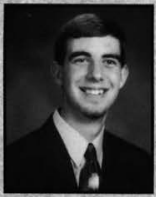

Michael Richandson

Electrical Engincering

Eiger, $\mathrm{CO}$

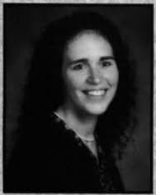

Geneva Roberts

Elementary Education

Milton, VI

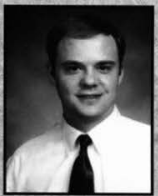

goel W. Rase

Multimedia Production Jamestown, $\mathrm{OH}$

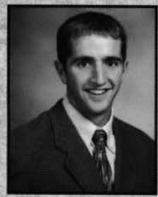

Michael Dean Reed

Business/Marketing Salem, OR

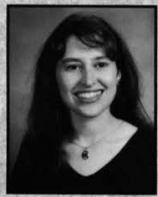

Saraf Louise Rittgers Nursing

Dallas Center, IA

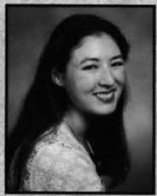

Jasmine Eve Rabinsont

Applied Psychology

Springfiedd, VA

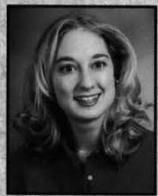

Kristin Diane Rosner

English Education

Herndon, VA

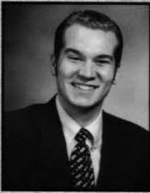

David Reisenbigler

Sociology/S.S./Psychology Eric, IL

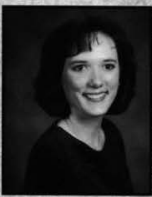

Yamie Maric Ritzer

Nursing

Eagle River, W]

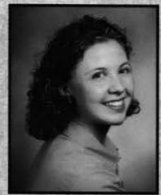

Mindy Robinson

Music Education

St. Louis, MO

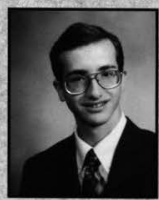

Brian Rosseau

Bible Comprehensive

Warner Robins, GA

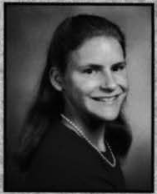

Kelly x. Reitz

End User Info. Systems

Lebanon, N]

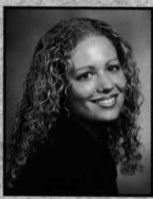

Hannafi Rachiel Rivers

Music Education

Norch Haverhill, NH

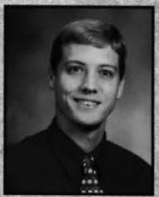

Ryan Roesler

Cam. P.E./Sport Manage.

Douglassville, PA

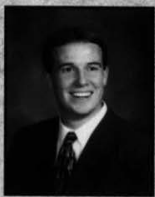

Douglas M. Rotmant

Finance/CIS

Cedarville, $\mathrm{OH}$

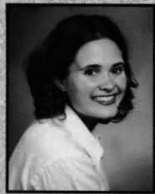

Racfiel Reno

English Education Edmond, OK

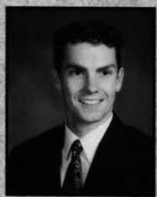

Gidam Rizer

Psychology

Virginia Beach, VA

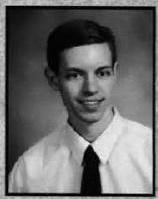

Adam Rorex

Criminal Justice

Gardena, CA

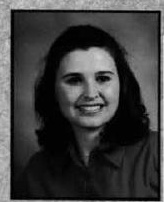

Maranathia Lee Rubery

Chemistry

Marthews, IN 


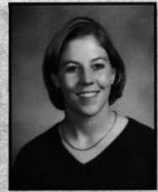

Casey Lee Ruffin

Professional Writing

Grand Lakc, CO

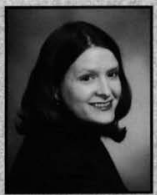

Krista D. Saul

Acoounting

Huber Heights, $\mathrm{OH}$

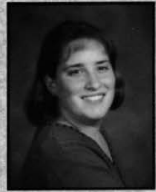

Sarafin go Schificher

Elementary Education

Springfield, $\mathrm{OH}$

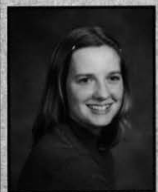

Stepfranie Sefint

Nursing

Xenia, $\mathrm{OH}$

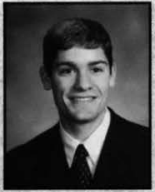

Scott D. Rufilman

Biology

Cedarville, $\mathrm{OH}$

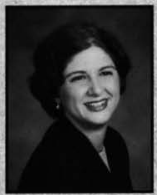

Gretcfien T. Savagge

Nursing

Whitinsville. MA

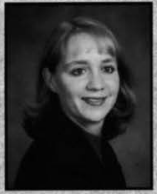

Lisa Jean Scfirock

Chemistry

Atmore, AL

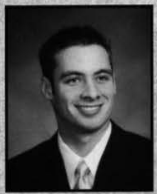

Daniel $P$. Sellers

Biology

Columbus, $\mathrm{OH}$

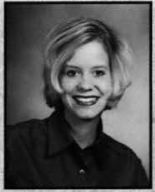

Megan 9. Ruoss

Nursing

Reading, PA

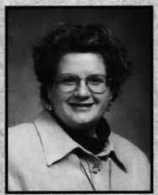

Melanie L. Scheerschunidt

Professional Writing

Jamestwon, $\mathrm{OH}$

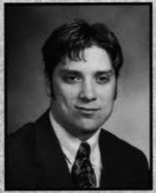

Dean Scfuler

Mulrimedia Technology

Horscheads, NY

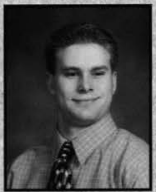

Sflane R. Sevo

Mechanical Engineering Belleville, MI

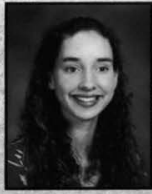

Rachiel Efizabeth Ryan

Nursing

Delaware, $\mathrm{OH}$

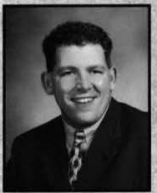

Joel Schiente

Pre-Seminary

Grandville, MI

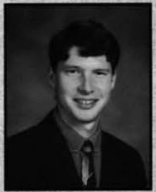

Ieffrey Schumactier

Mechanical Engineering

Pandora, $\mathrm{OH}$

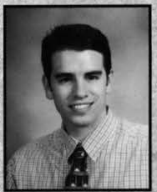

Maron Sfiow

Mechanical Enginecring South Paris, ME

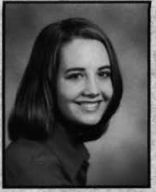

Amanda Sage

Applied Psychology

Fremont, $\mathrm{OH}$

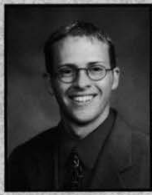

Gabriel Daniel Schlappi

Finance Marketing

Cedarville, $\mathrm{OH}$

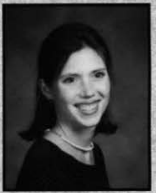

Connie Scfiwartz

Elementary/Special Ed.

Horsham, PA

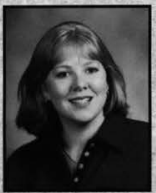

gill Shiearer

Education

Indianapolis, IN 


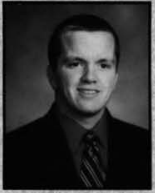

C. Jeremy Sfielford

Org. Communications

Naples, FL.

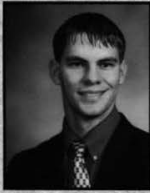

Josfua Stanley Sfierwood

Bible Comprehensive

Pensacola, FL.

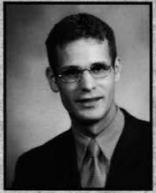

Micfiad Sfiepfierd

Bible Preseminary

Clarence, NY

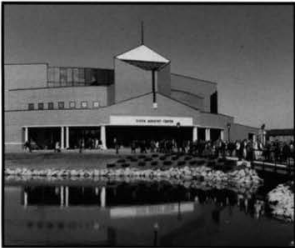

$-C-A-M-P-U-S$. changes

In their four years of college, the senior class watched the Cedarville College campus change and grow dramatically. They experienced the final days in the old Jeremiah Chapel, where students signed into chapel on a clipboard and coined the term, "slash and dash." They also experienced the enormity and beauty of the Dixon Ministry Center and new Jeremiah Chapel. They witnessed the end of Bethel and the addition of the McKinney, McChesney, Johnson, and St. Clair dorms. Yet, the change that they will probably remember most is that they were the last class to graduate from Cedarville College. Despite the changes, it was still true that Cedarville remained true to the Word of God and the testimony of Jesus Christ.

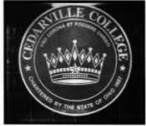

The Cedarville College seal in Alumni Hall.

The Dixon Ministry Center provides an enjoyable chapel experience for all.

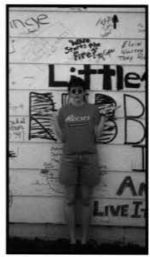

Tif Cope celebrates the end of Bethel at the graffiti party.

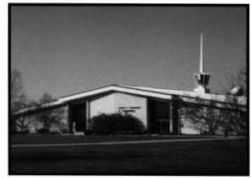

The old Jeremiah Chapel now has a new look with the Apple Technology Resource Center.

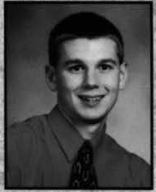

Xen Sfiterman

Electrical Engineering

Eagle Creck, OR

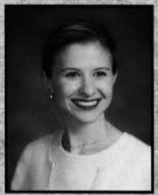

Erin Diane Sfipp

Nursing

Kansas City, KS

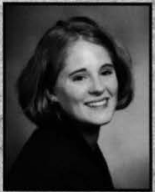

Rachel D. Stider

Nursing

Dayton, $\mathrm{OH}$

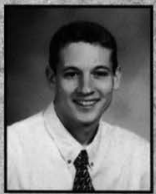

Chris Smith

Mechanical Engineering

Cambridge, OH

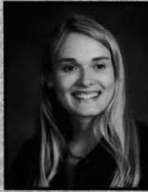

Kinberfy Ants Shilton

Nursing-Cross Cultural Cedarville, $\mathrm{OH}$

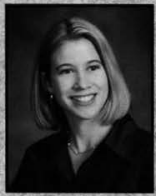

Cfiristian Joy Sicard

Special Education Hampton, NH

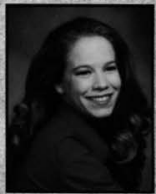

Brittany S. Smith Marketing Brighton, MI

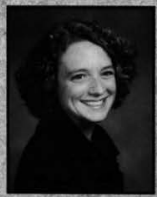

gaclyn Marie Smith

Marketing

Elmer, NJ 


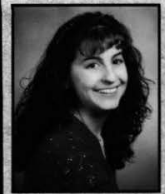

Kristie Smith

Elementary Edacation Pennsville, N]

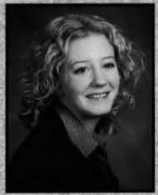

Anna Spears

Elementary Education

Carmel, IN

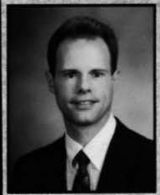

Eddic Steetie

Bible Comp./Pastoral/C.E.

Dayton, $\mathrm{OH}$

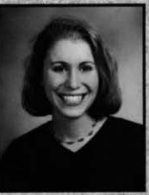

Any A. Stowers

Flementary Education

Coloma, MI

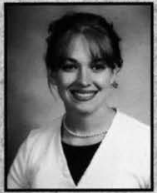

Sharon Smith

Elementary Education

Madison, AL

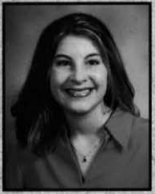

Cisa Rence Stacffiouse

Communications

Xenia, OH

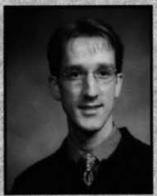

Vatfian Stepfiens

Applied Psychology

Sebring, FL

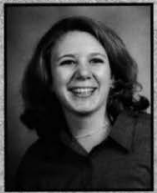

Mawren Strang

Sociology

Pitusgrove, NJ

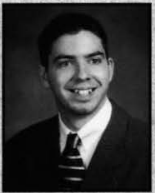

Stepfien 9. Simith

Elementary Educarion

Miamisburg, $\mathrm{OH}$

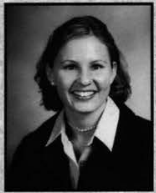

Iernifer yo Stanfiewice

Spanish

Indianapolis, IN

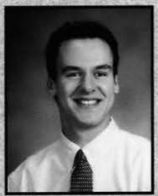

Ryan Christopher Stevens

Accounting

Lincoln, ME

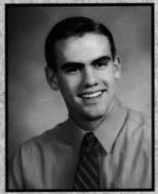

Dariel Strang

Bible

Cedarville. $\mathrm{OH}$

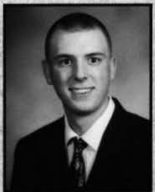

Dale W. Sorensen

Mechanical Engineering

Warren, PA

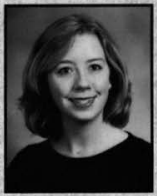

Tamuny Betfi Stark

International Studies

Cedarville, $\mathrm{OH}$

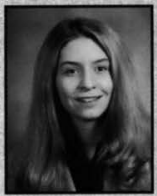

Heidi Lynn Stevenson Marketing

Springfield, $\mathrm{OH}$

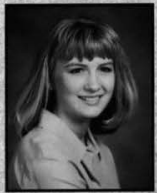

Jennifer Strong

Nursing

Cedarville, $\mathrm{OH}$

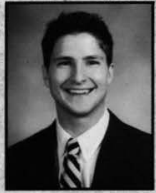

Ryan M. Sparzak

Pre-Seminary

Grandville, MI

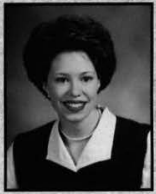

Susanna Steeg

Elementary Education

Jackson, WY

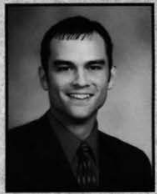

Wilfiam Howard Stewart

Christian Education Youth

Cincinnati, $\mathrm{OH}$

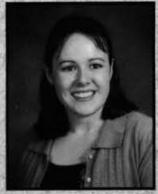

Kendra Amn Strong

Office Systems

Utica, MI 


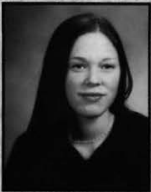

Jenny Stryfer

Finance

Barre, VT

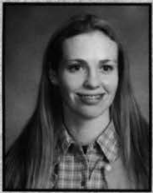

Tara M. Swaney

English

Prospect, $\mathrm{OH}$

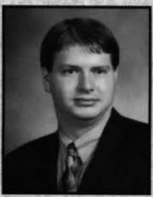

gay R. Talladay

Mechanical Engineering

Milan, MI

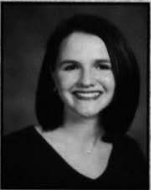

Katrina Thayer

Nursing

Oakland. MD

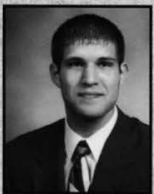

Ben H. Stutzman

CIS

Bartow, FL

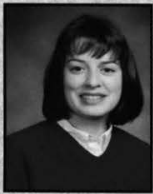

Ann Marie Swart:

Applied Psychology

London, $\mathrm{OH}$

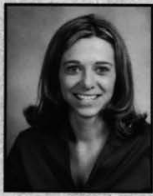

Teresa Tallarita

Pre-Law

Jolict, IL

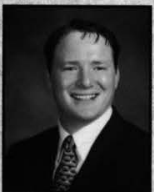

David $\%$. Thigpen

S.S./Histoóry/Education

Codarville, $\mathrm{OH}$

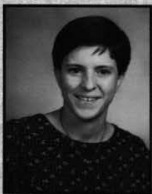

Becky Summers

Physical/Health Fducarion

Bainbridge, PA

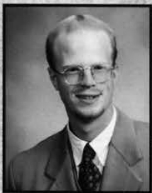

Paul Swerison

Social Science Comp.

Barton, VT

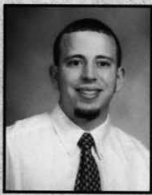

Scott Micharel Tatium

Marketing

Hartville, $\mathrm{OH}$

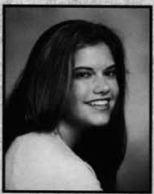

Nicole Renee Thomas

Music Education

Flint, MI

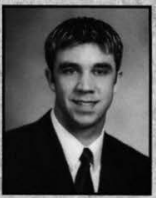

Erie Svendsen

Multimedia/Broadcasting Algonquin, IL

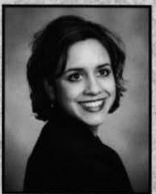

Tonya Marie Swiger

Elementary/Special Ed. Xenia, OH

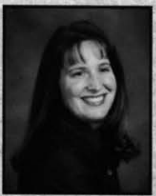

Stacy Tayfor

Elementary Education

Ephrata, WA

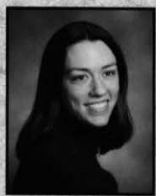

Sarafi $L$. Thomas Athletic Training Wyckoff, N]

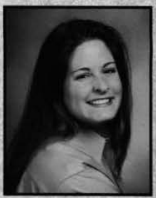

Sandra Swales

Applied Psych./Counseling Onillis, Ontario

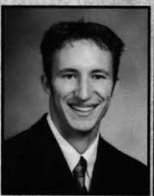

Douglas James Tait III Mechanical Enginecring Walker, MI

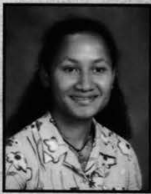

Rebecca Thamgan

English

Colonia, YAP

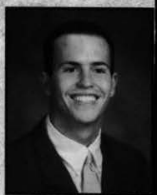

Ben M. Thompson

Marketing

Solon, $\mathrm{OH}$ 


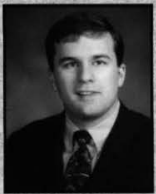

Yonathan Tumer

English

Roanoke, VA

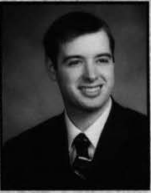

Ryan M. Urbassik

Mechanical Engineering

Menomee Falls, WI

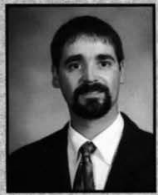

Veif O. Vitafi

Bible Comprehensive

$\mathrm{Xenia,} \mathrm{OH}$

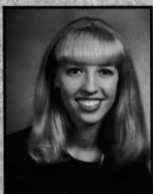

Melanie Wadlington Nursing

Miamisburg, $\mathrm{OH}$

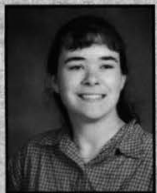

Amy Twigg

Special Edueation

Martinsburg. WV

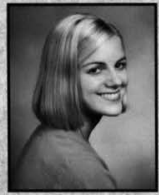

Heather Rnn Van Gorp

Nursing

Indianapolis, IN

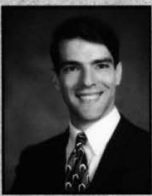

Jared Micfinel Voigt

Mechanical Engineering

Midland, MI

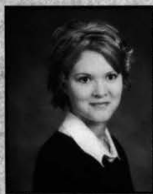

Jessica Wagner

Music Theory/Composition Haywood, VA

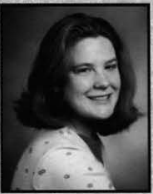

Esther Twigg

Elementary Education

Norwood. $\mathrm{OH}$

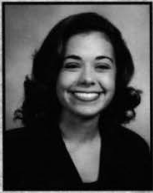

Krista Voelle Van Hooser Psychology

Ankeny, IA

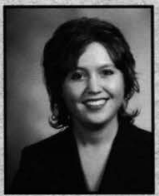

Rachiel Volpe

Marketing

West Seneca, NY

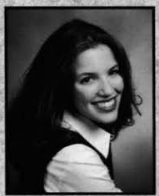

Eindsay Wagner

Nursing

Gig Harbor, WA

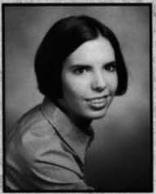

Carrie Tyson

Finance/Accounting

Boothwyn, PA

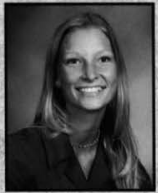

Michiefle Lymn Vanfier

Physical Education

Pittsgrove, NJ

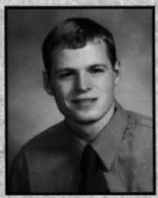

Ty Waardenburg

Criminal Justice

Nashua, NH

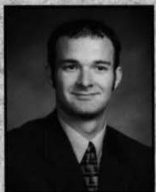

Scott Walker

Bible Comp/C.E. Yourh Cedarville, $\mathrm{OH}$

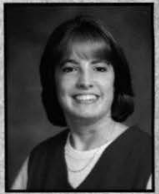

Heatfiet Tyson

Elementary Education

Millerstown, PA

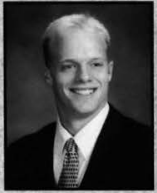

Natfian Pfillip Verwyys

Business Management

Grand Rapids, MI

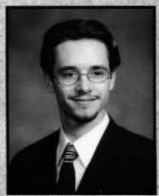

Michael Wade

Electrical Engineering

Whitefish, MI

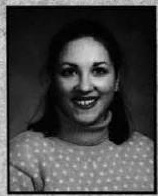

Jemnifer L. Wallace

Prof. /Tech. Writing Com Duncansville, PA 


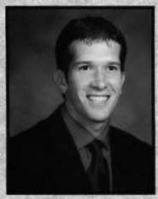

Braden Wingert

Athletic Training

Mechanicsburg, PA

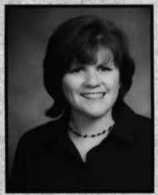

Betfany 9. Withers

American Studies

San Dicgo, CA

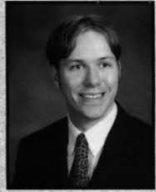

Natfian Wright

Electrical Enginecring

Tyler, TX

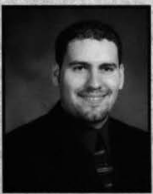

Kevin Matticew Young

Bible/Broadcasting

Connersville, IN

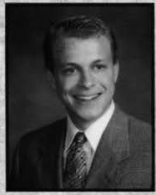

Jonatfian Winn

C.E./nternational Business Kentwood, MI

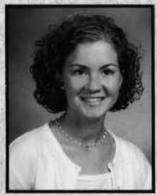

Elizabeth Wolfe

Marketing

Ligonier, IN

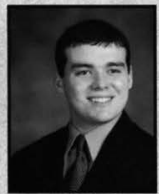

Mark Ran Wyse

Mulcimedia Technology

Woodstock, II

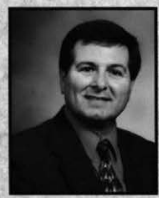

William E Yowler

Bible/Pastoral Studies

Springfield, $\mathrm{OH}$

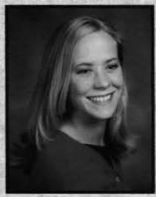

Yamie Dianne Wisfart

Communications

Canton, MI

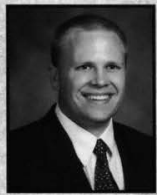

Jan-Fann Wolters

Electrical Enginecring

Belmont, WI

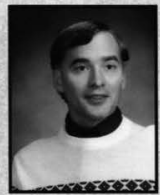

Cfris yoder

Undeclared

West Liberty, $\mathrm{OH}$

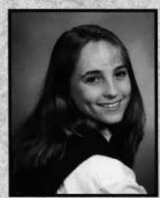

Jodi Lynn Zell

Nursing

Cedarville, $\mathrm{OH}$

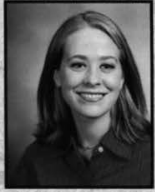

Jennifer Lynne Wistiart English

Canton, MI

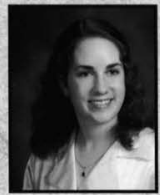

Krista Word

Electrical Engineering

Crofton, MD

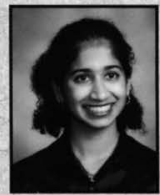

gaya Yofiannan

Narsing

Cedarville, $\mathrm{OH}$

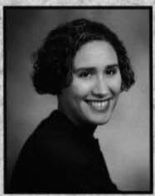

Amy S. Zimnerman

Cross-Cultural Nursing

Oostburg, WI

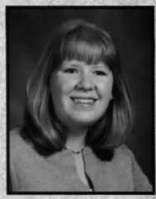

Katie Wolf

Music Education

Lirtleton, CO

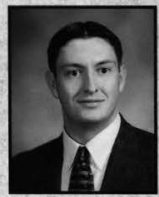

William Garnett Wotring Christian Education Youth Lansing, MI

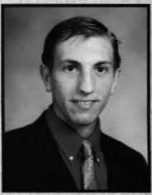

Vathan KarlYorgey

Biology

Boyertown, PA

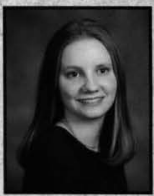

Mary Zuleger

Elementary Education Appleton, WI 


\section{$-\mathrm{S}-E-\mathrm{N}-I-\mathrm{O}-\mathrm{R}-$ sponsor}

Jeff Beste, husband, father, Assistant Director of Campus Activities, and musician was the sponsor of the class of 2000. Most students knew Jeff as one of the members of the staff that sang on stage during Bible conferences. Some knew him for his involvement in worship at Grace Baptist Church in Cedarville. The members of Theta Rho Epsilon knew him as their advisor. Most important to Jeff was that his wife Shelley and his daughters Kylie and Sara knew him as husband and father.

In 1982, Jeft joined Cedarville's student body, planning to stay only one year. He quickly changed his mind after he got to know other students and saw them "excited about their relationship with the Lord." He graduated in 1987 and taught middle school math for one year. In 1988, Jeff came back to Cedarville College to work in the admissions office.

Jeff worked in the admissions office for four years then became a Resident Director in both the
Hill and McChesney. For one year, he served as Director of Community Ministries, and in 1998 he became the Assistant Director of Campus Activities. In the Campus Activities Office, he organized concerts, supervised the snack shop on the second floor of the Athletic center, and worked with student organizations to develop leaders.

As the senior sponsor, Jeff oversaw class activities and made himself available as a resource to the class. He said, "It is fun getting to know the officersand the students." He enjoyed "watching the students grow over four years... It's hard to see them graduate" he said, but "email is a wonderful thing." The convenience of e-mail helped Jeff to keep in touch with the many students that he had gotten to know in his seventeen years at Cedarville. Hegained many friends as the sponsor of the class of 2000 , and Jeff was excited about using email in order to continue contact with the students with whom he had become friends.

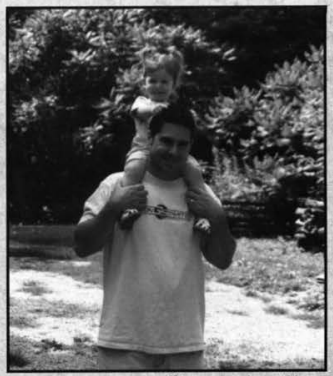

Time spent with his daugter Kylie is one of Jeff's favorite things to do.

"Jeff is someone that could make me laugh, encourage me to grow, and help me make tough choices," states Kelsic Perkins.
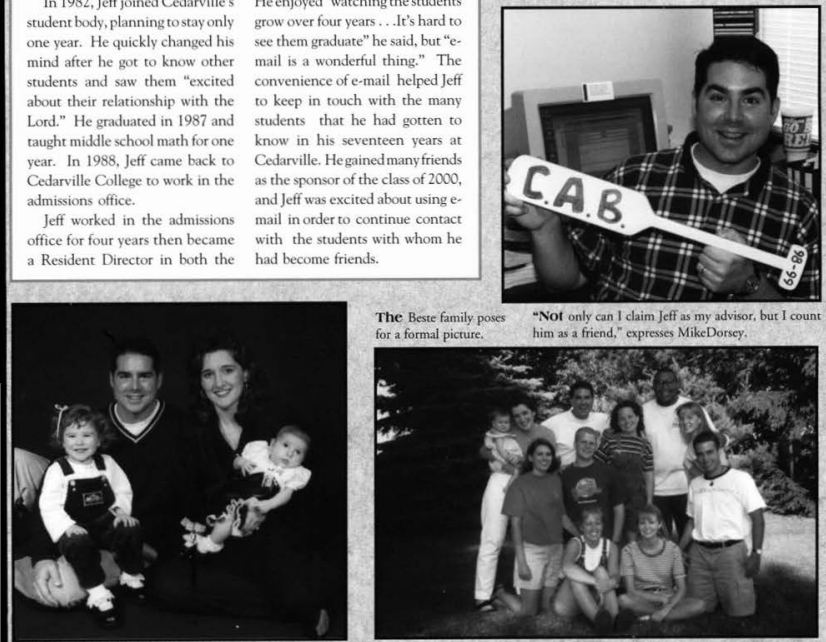

The Beste family poses

"Not only can 1 claim Jeff as my advisor, bur I coune for a formal picture.

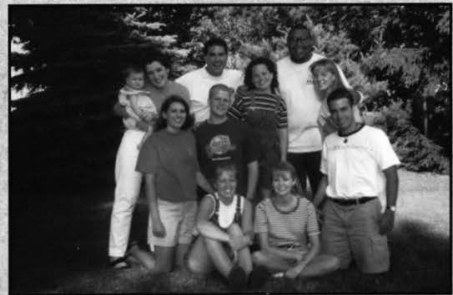


Natalie Grant enjoys the J/S Banquet with her husband, who is also her accompianist.
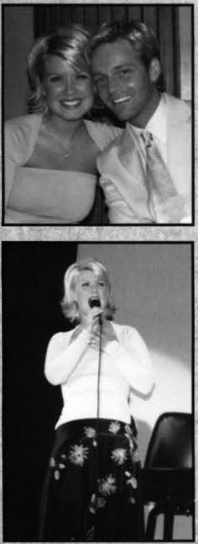

Praising the Lord is the focus of Nanalie Grant's music and entertainment.

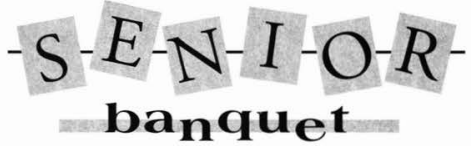

The Junior/Senior Banquet was held at the Omni Netherland Plaza Hotel in Cincinnati with singer Natalie Grant providing the entertainment. Getting from Cedarville to Cincinnati was a problem for many students, whofound themselves stuck in traffic due to road construction. "We were more than an hour late," said junior Kevin Hamilton. "Fortunately, they

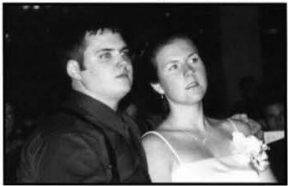

Students are amazed by the music and talent of Natalie Grant. ity of students remembered about the evening. "I'll think back about how gorgeous my girlfriend was," said senior Josh Sherwood, "and how much fun it was to spend my last JS with her and my friends?" Jonathan Winn agreed, saying, "JS gave me a chance to show off my fiancee for the first time. It was really neat to have a formal time to spend with her and my friends."

Although the were running behind schedule anyway." Still, most attendees arrived in time for dinner. "We met my goal for this year: I was there before they started serving dinner," said senior Denelda Foltz. "I haven'tdone that for the past two years."

Spending one last time with friends and significant others is what the major- senior memories slide show had some rechnical problems and had to start over, the students took it in stride. Prior to the slide show, Natalie Grant, accompanied on keyboard by her husband, sang for about an hour. As senior Scott Lehr said, "It was a pretty good evening overall for everyone involved."
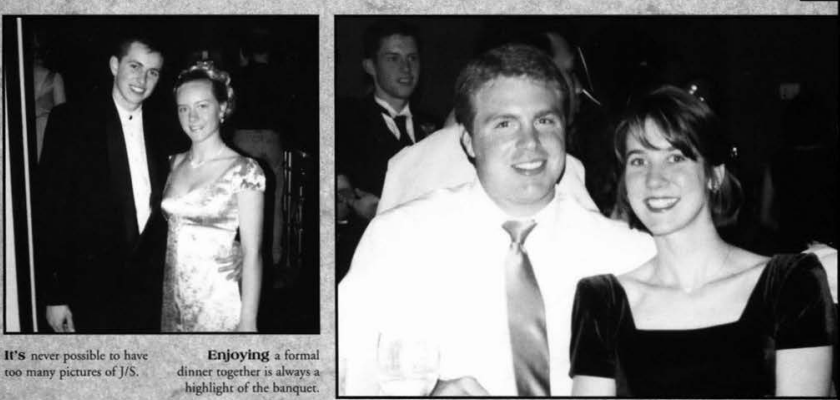

It's never possible to have too many pictures of J/S.
Enjoying a formal dinner together is always a highlighr of the banquet. 


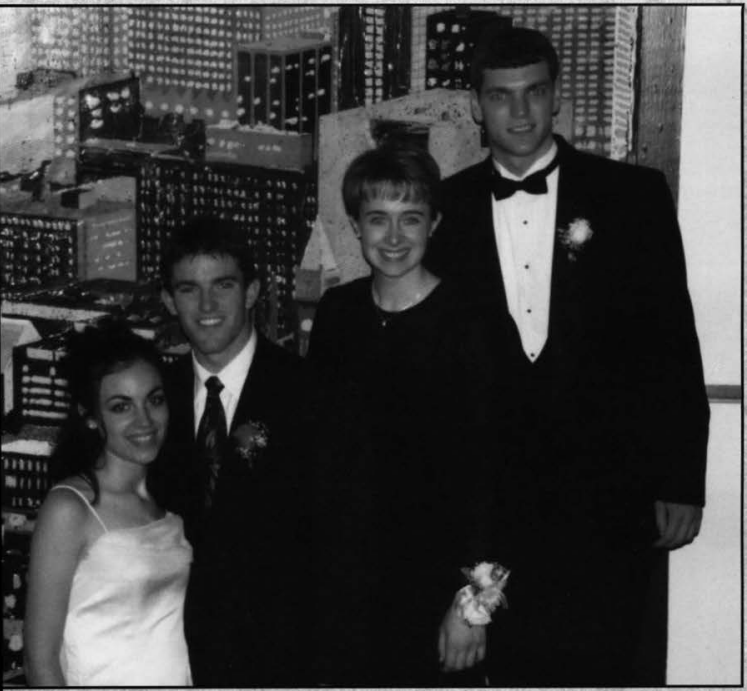

Many J/S attendees find the majestic staircases of the Omni Netherlands Hotel a great location for pictures.
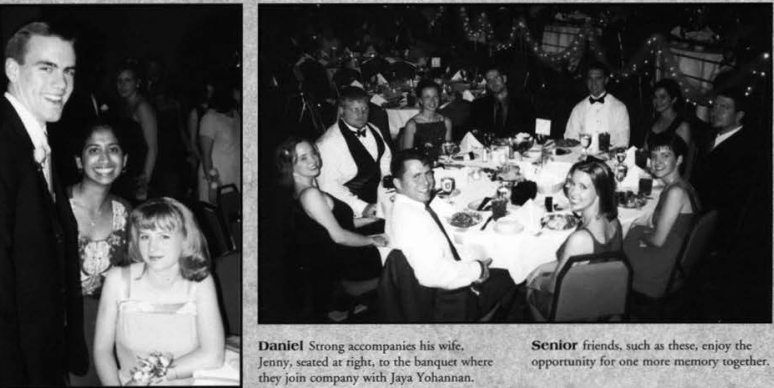

Daniel Strong accompanies his wife, Jenny, seated at right, to the banquet where they join company with Jaya Yohannan.
Senior friends, such as these, enjoy the opportunity for one more memory together. 


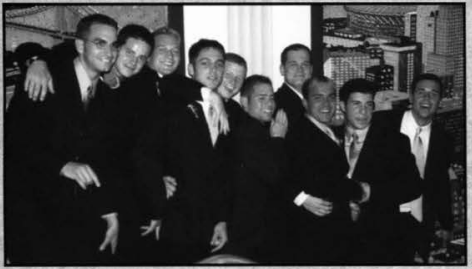

Nothing beats an

enjoyable evening

with the guys.
Many couples find $\mathrm{J} / \mathrm{S}$ a memorable occasion of spending rime together.
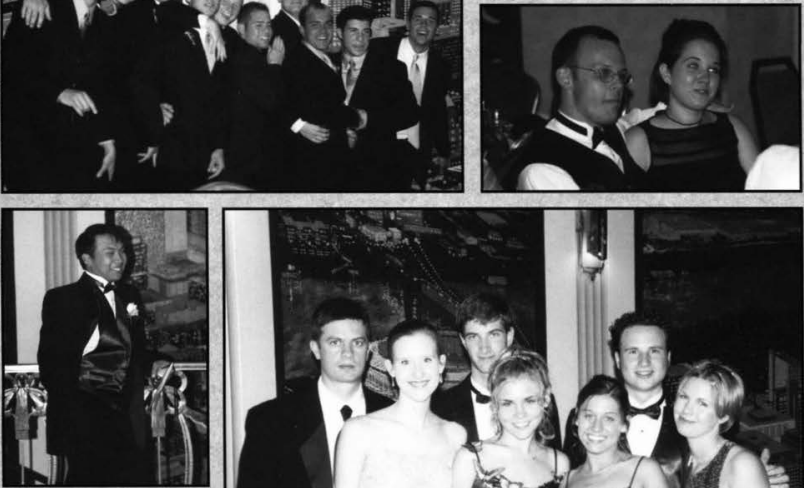

Looking suave, this

student watches the view

from the balcony.

All over the hotel, groups of people taking pictures are found.
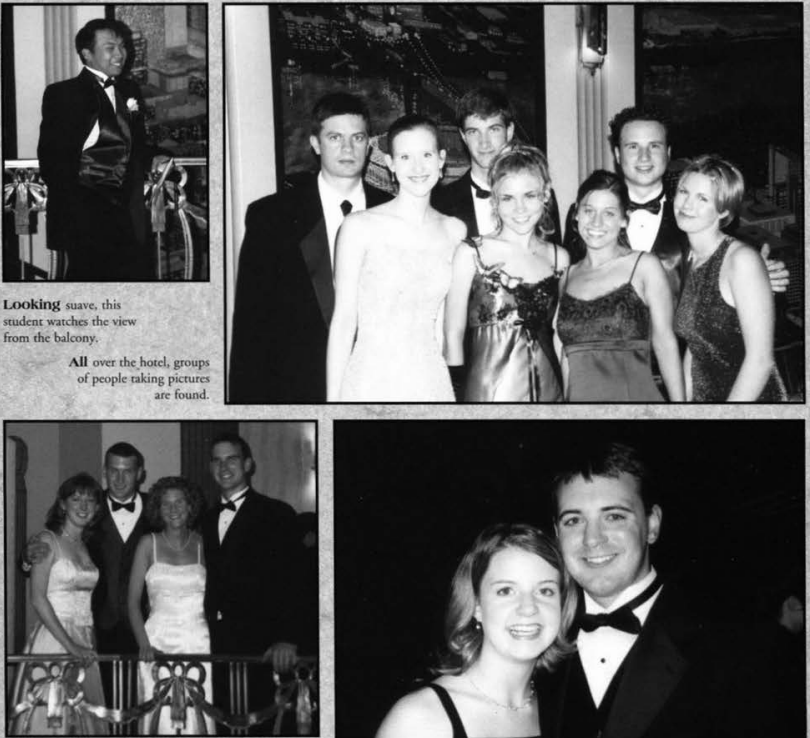

Seniors Emily Bohl and Christa Hill spend a fun-filled evening with their dates. 118
Katie Little and Michael Gregg make the perfect couple.

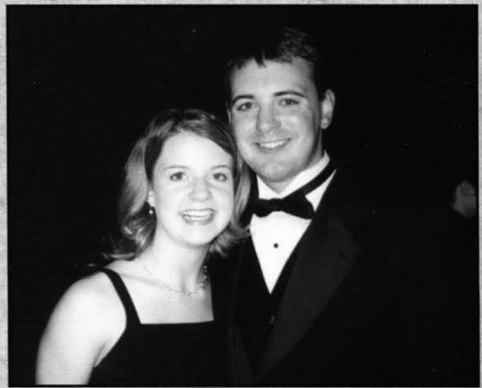



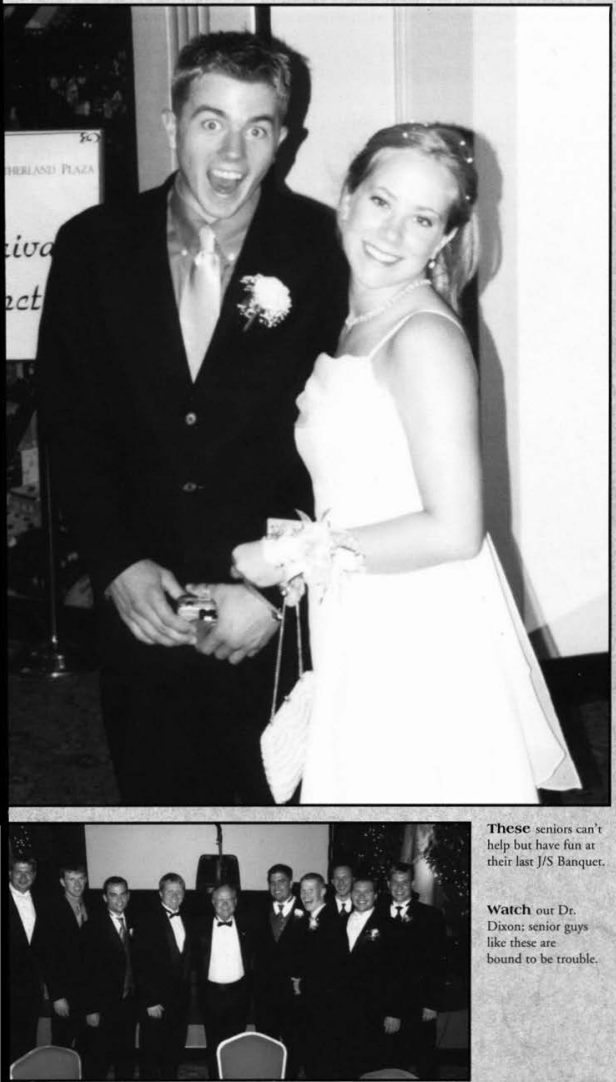

These seniors can't help but have fun at their last J/S Banquet.

\section{Watch out Dr.}

Dixon; senior guys like these are bound to be trouble.
Two beautiful women

will make any man

smile like this one.

Esther Kelly is enjoying the evening with her date. (below middle)
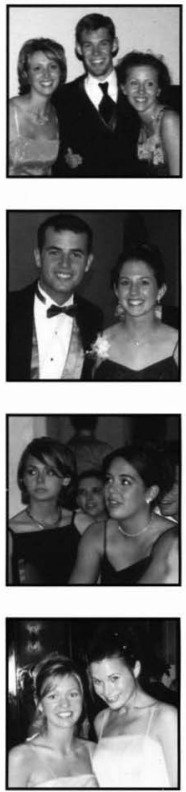

Intent on the music, many students find themselves wishing for a longer concert. (above middle)

Mandy McGuire and Lindsey Borland put on their best smiles. 
There are many tasty snacks and drinks for the seniors to have at the reception.
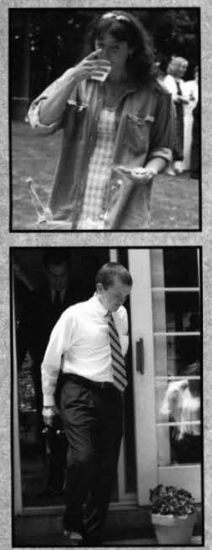

Jeremy Shelford gets a tour of the house.

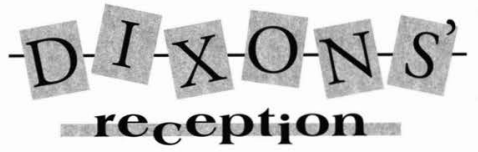

Seniors by the score converged at the Dixon home for the annual senior reception on May 21. Dressed in their Sunday best, they met on the lawn and munched on crackers, cheese, and cookies from Chuck's. They also were able to tour part of the Dixons' house. "I enjoyed it," said MichaelBoehm. "A phrase 1 kept hearing

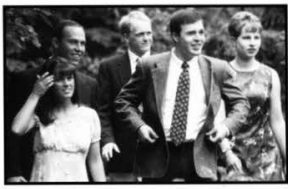

These seniors try to take in all the action while at the reception. home atmosphere. I did think it was odd that we had to take our shoes off before we went inside."

Students thought it was interesting to see the everyday minutia of the president's home. Kyle Kickbush found himself noticing the driveway. "I saw Dr. Dixon's Jag sitting outside, and it was actually dirty," said Kickbusch. "That surprised me."

Kyle Gantz was impressed that the Dixons made an effort to socialize with the people repeat was, 'I can't believe it's our turn to do this. The four years have gone so fast."

Although Dr. and Mrs. Dixon spent a solid three hours mingling with seniors; it didn't seem to faze them. "They were really friendly," said Lindsey Lorinovich. "It was interesting to see them in their seniors individually. "It's nice that he is personally interested in the seniors and invites them to his house, even though the student body keeps getting bigger," he said.

Overall, students enjoyed the low-key re. ception and opportunity to talk with friends. "It was a really nice way to close the year," Lorinovich said.

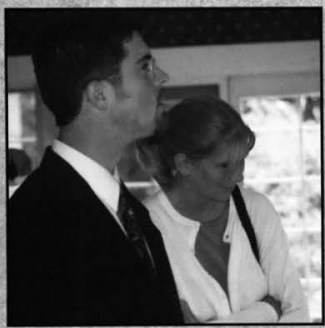

Look but do not touch is the rule inside the Dixons' ornatehouse.
Brittany Jones, Jamie Baker, and Julic Lamborn enjoy visiting the Dixoris' together.

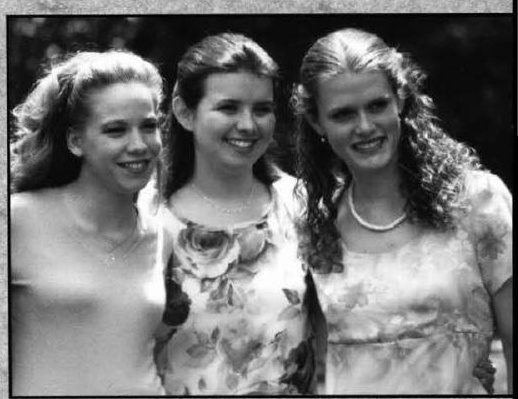



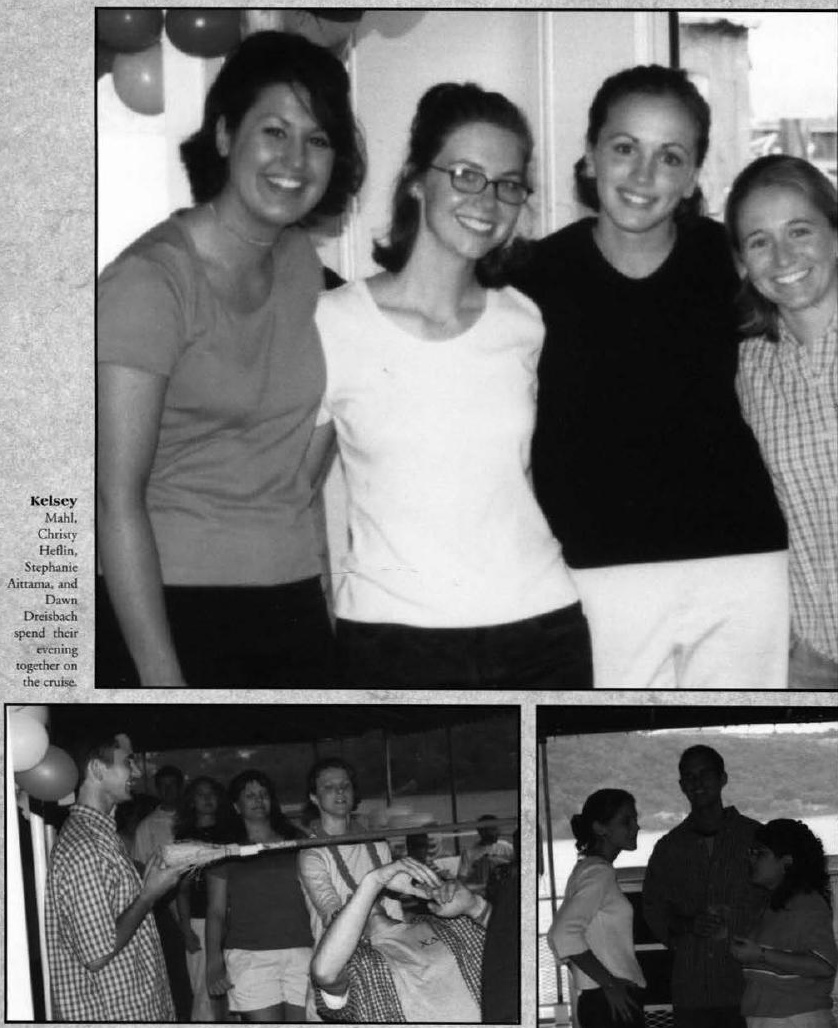

Who knew that seniors about to graduate could have so much fun doing the Limbo?

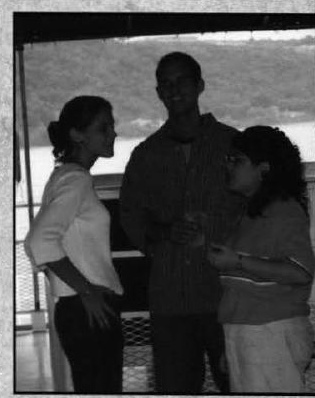

Reminiscing old times, Sarah High, Grady Peeler, and Carla Clark share Cedarville memories. 


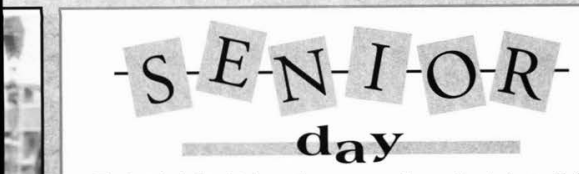

The Saturday before their last under. graduate exams, seniors took a day for themselves. Senior Day was a time for seniors to say their preemptory goodbyes to friends, both old and new. Many seniors used the opportunity just to hang out one last time with their college buddies.

The seniors were able to buy discounted tickets to King's lsland, where the newest ride was "Son of Beast." The roller coaster was wooden and intended to be an even better ride than the original "Beast." Many se-

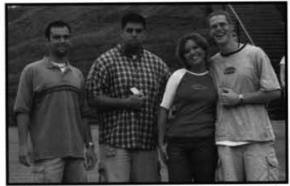

Bill Stewart, Matt Plaatje, Rachel Volpe, and Chris Headings are overjoyed to be at Senior Day. traveling to the riverboat. Unfortunately, they missed the cruise but had an opportunity to wave to their classmates as the boat later passed underneath the bridge.

Throughout the evening, seniors enjoyed eating and reminiscing about their nearly finished college days. A birthday cake for Shelley Beste, wife of senior advisor Jeff Beste, was part of the festivities. After dinner entertainment included games on the upper deck such as the limbo niors rook this opportunity.

At 6:30 the seniors began boarding a riverboat for a dinner cruise. Some students had to battle traffic in order to arrive on time. Several students were even involved in a car accident on a bridge crossing the Ohio River, while and musical chairs. Live musical entertainment was provided by Cedarville College students.

Despite a downpour of rain towards the end of the cruise, the trip was a memorable and enjoyable evening for everyone.
Heather Van Gorp and Anna Spears watch the limbo contest from the sidelines,
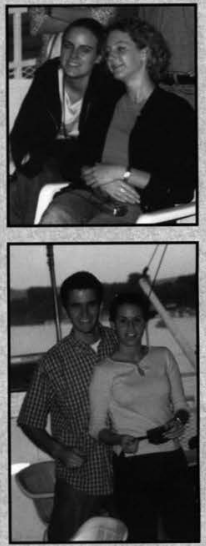

Jamie Hall poses with a friend for a picture souvenir of their trip. on the Ohio River.
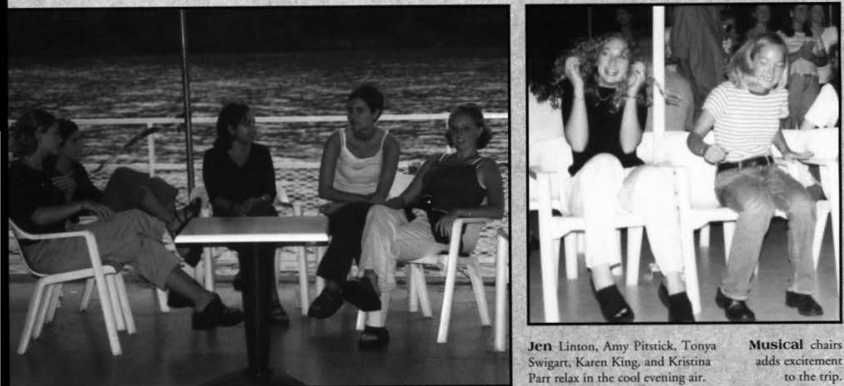

Jen Linton, Amy Pitstick, Tonya Swigart. Katen King, and Kristina Parr relax in the cool cvening air.
Musical chairs adds excitement to the trip. 
Dr. Dixon emphasizes the Word of God to graduates.
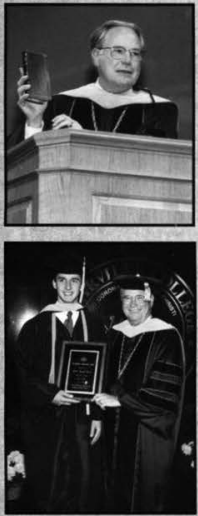

David Fretz is honored with the Faculty Scholarship Award.

Scott lehr is recognized for his leadership at Cedarville.

\section{$-1-0-4-t-h-$ \\ commencement}

"Do you think it will rain today?" a friend asked Dr. Dixon before the 104th Annual Commencement at Cedarville College. "No," he replied, "it never rains at Cedarville's commencement." Dr. Dixon's

"Don't quit on your spouse, your parents, chil. dren, or God."

Lonnie Nolt, a graduating senior who injured his leg in a motorcycle accident at the beginning of the school year and had to have it amputated prophecy came true as the Class of 2000 graduated on June 3,2000 .

The last class to graduate from Cedarville Col. lege before it changed to Cedarville University included 615 students. The Jeremiah Chapel

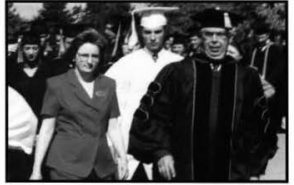

Dr. Matson leads the procession of graduates from the gymnasium to the chapel. has already learned this lesson. During Dr. Dixon's commencement speech, he congratulated Nolt on sticking to his studies during his recovery so that he could graduate as part of the Class of 2000.

Graduating senior Matt Plaatje said, "I'm so happy to have and overflow rooms held between 4,500 and finished. Of course I will miss all my friends, my 5,000 visitors.

Dr. Dixon passed on encouraging words to the graduates, including what would become the six most important days of their lives: birth, salvation, graduation, marriage, the birth of children, and the day students pay off their student loans. Dr. Dixon encouraged students not to quit. "Take the word quit out of your vocabulary." He said, professors who taught me so much, and the town of Cedarville. I never thought I'd say it, but Im really going to miss this place."

The college began a new tradition by handing out Bibles to all the graduates. Dr. Dixon said that with the final class graduating from Cedarville College, he wanted to emphasize the foundation of the college and importance of God's Word.

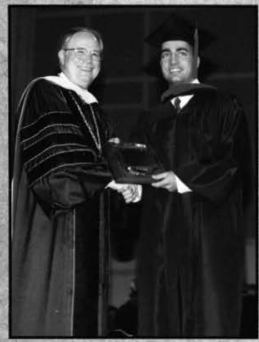

Alumni director Faith Linn distributes portfolios to the graduates.

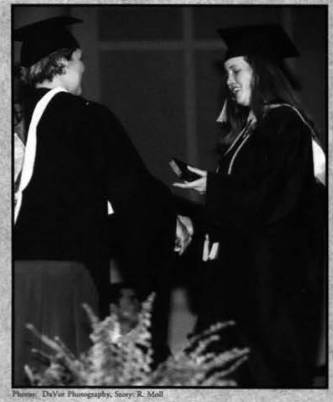

Dr. Dixon congratulates Kelscy Perkins on her award. 


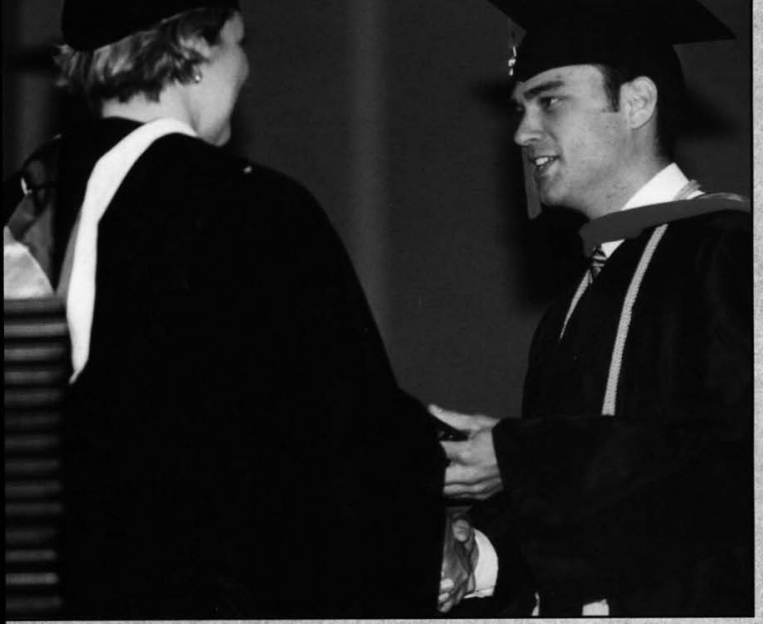

Graduates can't help but smile as they receive their degrees.
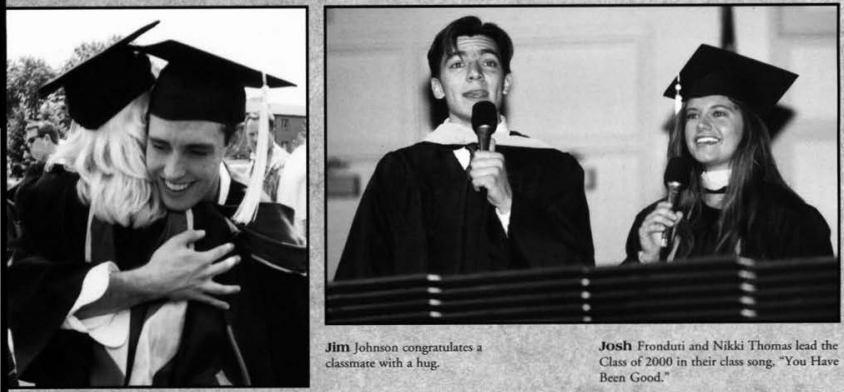

Jim Johnson congratulates a classmate with a hug.
Josh Fronduri and Nikki Thomas lead the Class of 2000 in their class song, "You Have Been Good." 
Ron Becker concludes his graduation walk with a photo by the American flag,

Candace Dodge is happy finally to receive her diploma as a member of the nursing class. (below middle)
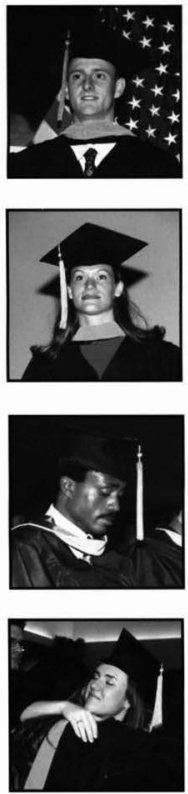

Sometimes the graduation ceremony can fill a senior with bittersweet memories. (above middle)

Jayme Brower congratulates a fellow nursing major on a job well done.

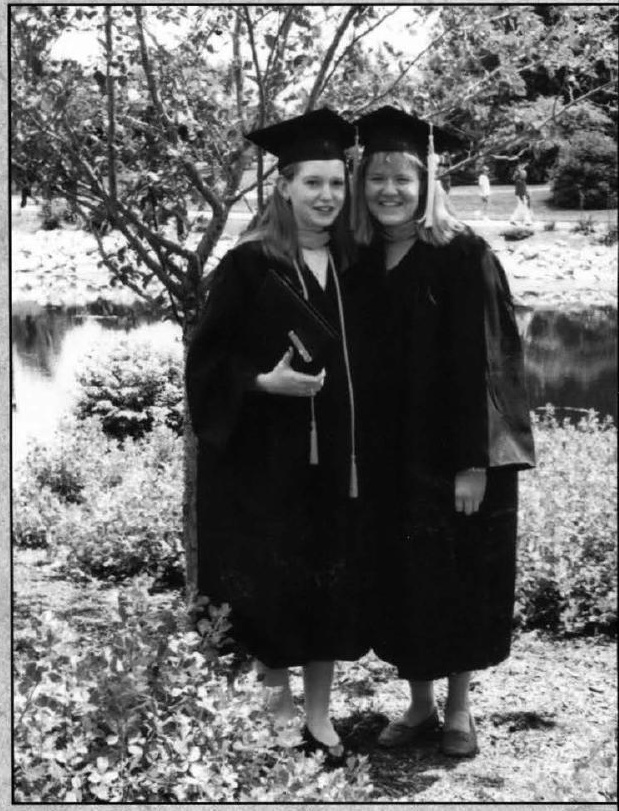

Amy Pool and

Vianna Kreuder-

Crowell pose in theit

graduation regalia.

Smiles are broad across the graduation plarform.

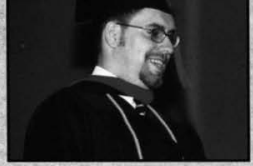

Lindsey Lorinovich applauds her fellow classmates.

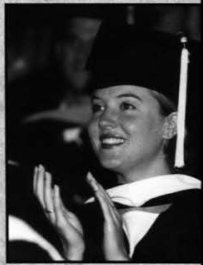


Campus Safery members congratulate the graduates as they enter the DMC.
Tammy Sark receives her $B . A$. in Biblical Education.
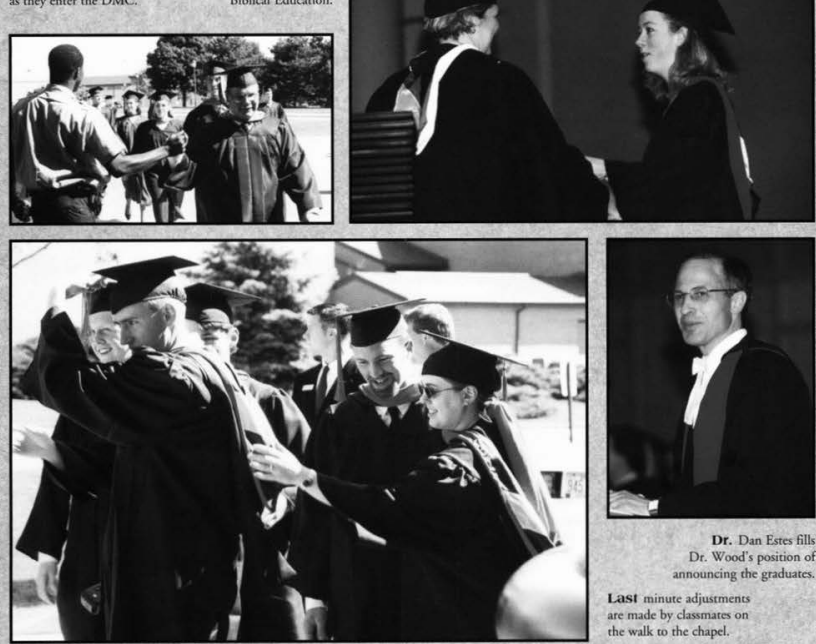

Dr. Dan Estes fills Dr. Wood's position of announcing the graduates.

Last minute adjustments are made by classmates on the walk to the chapel.
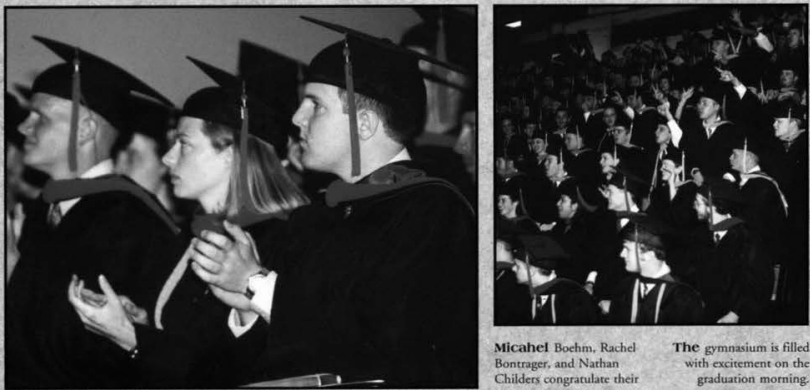

Micahel Boehm, Rachel Bontrager, and Nathan Childers congratulate their pecrs.
The gymnasium is filled with excitement on the graduation morning. 
Kevin Haisch and Tim

Kwast anticipate the enginecring convocation.
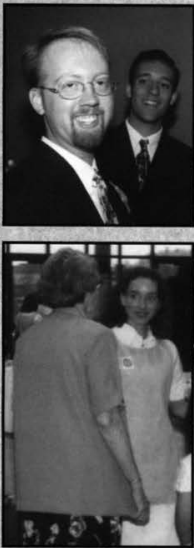

Rachel Ryan interacts with family members at the nursing reception.

\section{$-\mathrm{S}-E-\mathrm{N}-I-\mathrm{O}-R^{-}$ convocations}

The engineering and nursing convocations were held in the DMC the day before graduation. Alumni Hall was crowded with engineers, nurses, friends, and family as they gathered to commemorate the accomplishments of the manystudents who had finished their engineering and nursing degrees at CedarvilleCollege.

Outside the recitalhall, aband composed of engineering students and faculty played to entertain the many

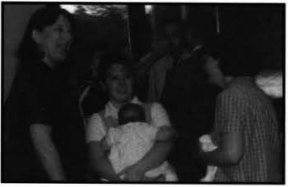

Dr. Baker and Kelsey Hintz, with her daughter, calk with a friend. gives us a sort of family time to be with those that we've spent the last four, sometimes five years with."

Dr. Zavodney opened the engineering convocation by highlighting the many events that the students witnessed during their time at Cedarville, including, "the big. gest non-event of all time, the y $2 \mathrm{k}$ crisis." Then Dr. Estes encouraged the graduating engineers, saying, "You are not engineers who happen to be Christians, but you guests. Graduating engineering student Matt Plaatje said, "It's a time to reflect on four years with friends and family and bring to close some of the best times of my life in a God-honoring way."

Nursing student Melissa House said, "It's an absolute relief. The convocation are Christians who happen to be engineers."

Dr. Dixon took time out of trustee meetings to speak at the nursing convocation. He thanked friends, families, faculty, and students for their endeavors, for their support and for their achievements.

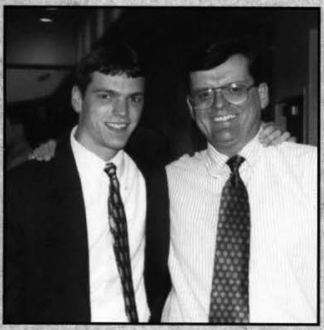

A photo with a favorite profecssor is a must for graduates.
Jim Billock, David Carr, Michacl Munro, and Adam Warnken sigh relief at being finished.

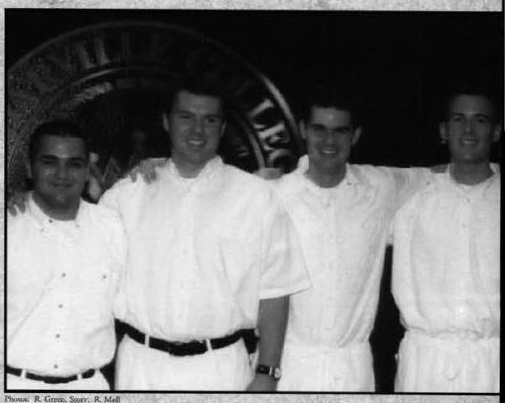



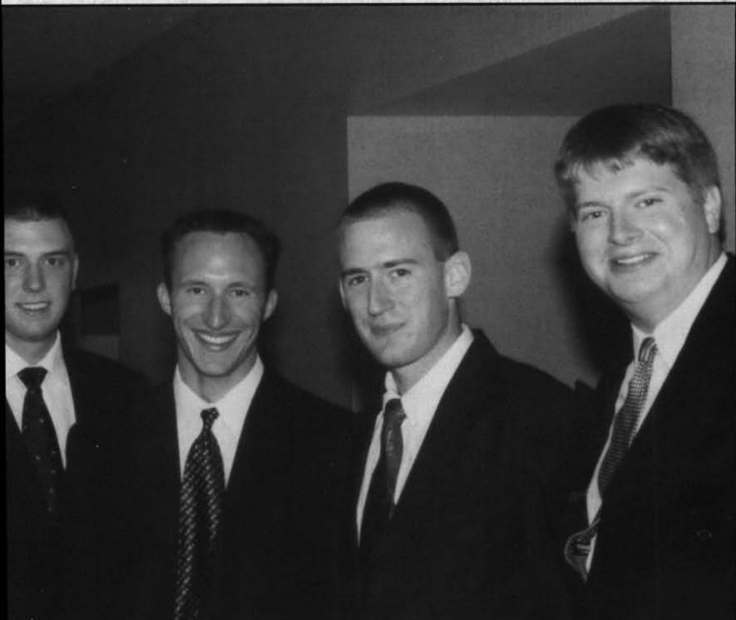

Dale

Sorensen,

Jason Covill,

Lonnie Nolt,

and Jay

Talladay are

excited to be

able to

celebrate this

day rogether.
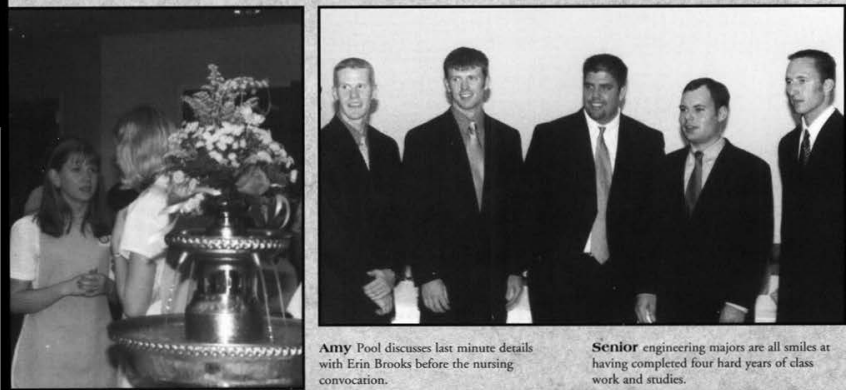

Amy Pool discusses last minute details with Erin Brooks before the nursing convocation.
Senior enginecring majors are all smiles at having complered four hard ycars of class work and studies.

$$
\text { 47. } x^{2}=\frac{129}{\text { SEsions }}
$$


... Gut men loved darkness instead of light because their deeds were evil. . . 


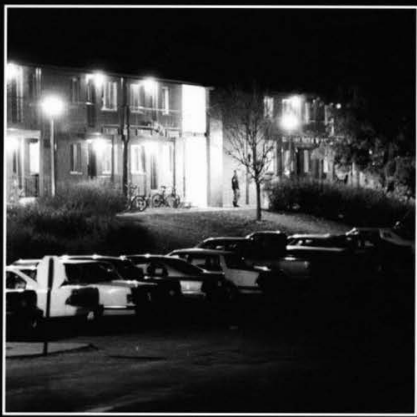

UNDERCLASSMEN 


\section{Juniops}

Class of 2001

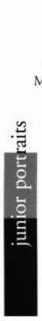

Tim Abbott
Christy Abbs
Jessica Ackerman
Michelle Ackerman
Bamidele Ajayi
Jared Aldridge
Rebecca Allen
Sarah Allen
Shawn Allen
Jewel Alley
Jared Applegate
Jacqueline Ayecte
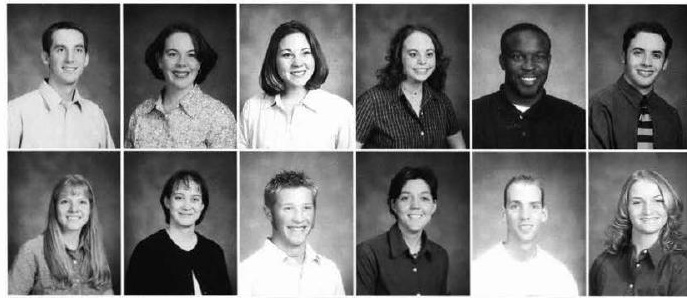

Liza Bacerra

Ruth Back

Jack Bailey

Vanessa Baker

Adam Ballah

Benita Banks

Ashley Barnes

Kelly Barron

David Barshinger

Abigail Beck

Deborah Beck

Karen Belknap

Eric Bender

David Bennett

Adam Beres

Diane Bielo

Kristina Bilodeau

John Bishop
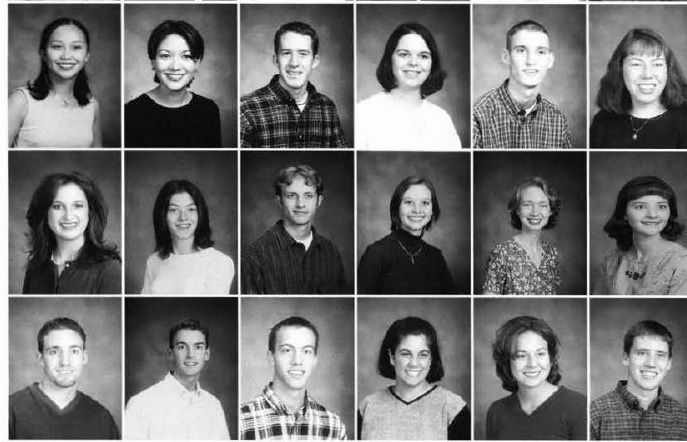


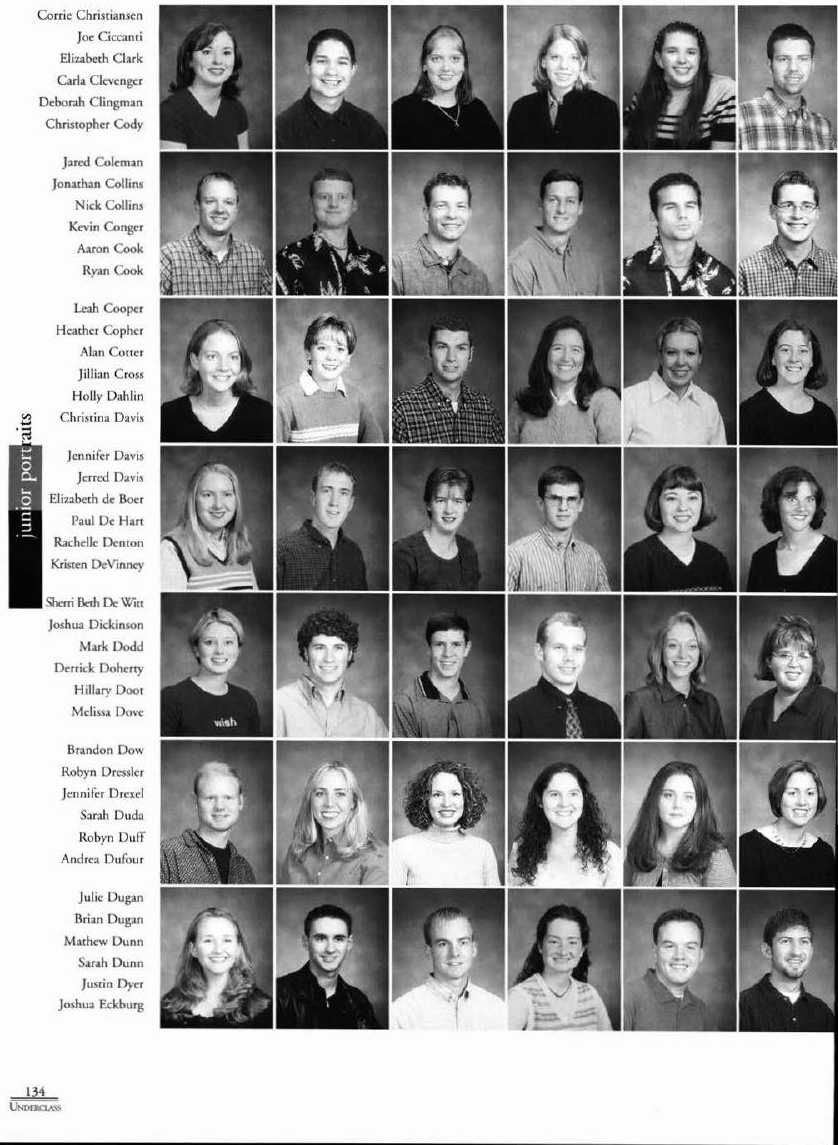




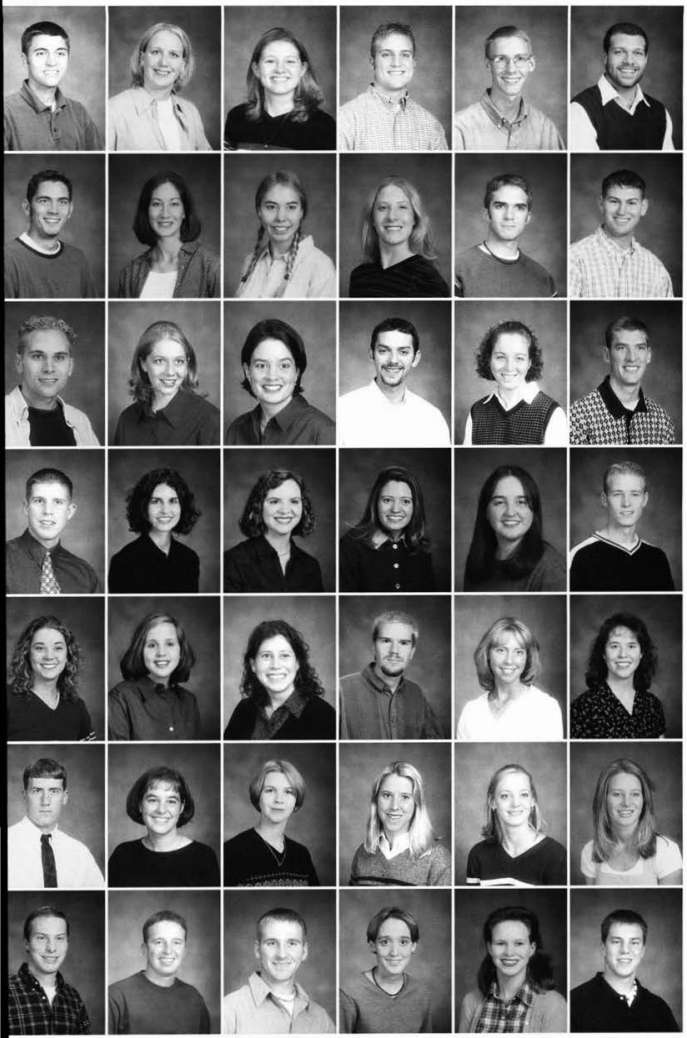

Jason Edcm

Codi Edgington

Trisha Edlund

Nathan Eikhoff

Ryan Einfeldt

Michael Einstein

Ryan Elam

Jenny Elliott

Danelle Ellis

Emily Elwell

Justin Epperly

Michael Essingron

Kerry Estes

Holly Favorite

Julia Fawcett

Michael Ferrigno

Amy Fenton

Timothy Fenton

Karl Feucht

Kelly Fisher

Julic Flick

Marisa Flory

Jessica Flynn

Chad Foerch

Melissa Foote

Kellie Forbes

Jenelle Forward

Dan Foster

Sarah Fraser

Leah Frazec

Mark Freeman

Hearher French

Katie Futrell

Lauric Gallo

Becky Garber

Heather Garie

Brian Garver

Benjamin Gayer

Sammy Gilbert

Vanessa Gilliam

Amanda Gillispie

Andy Gingrich 


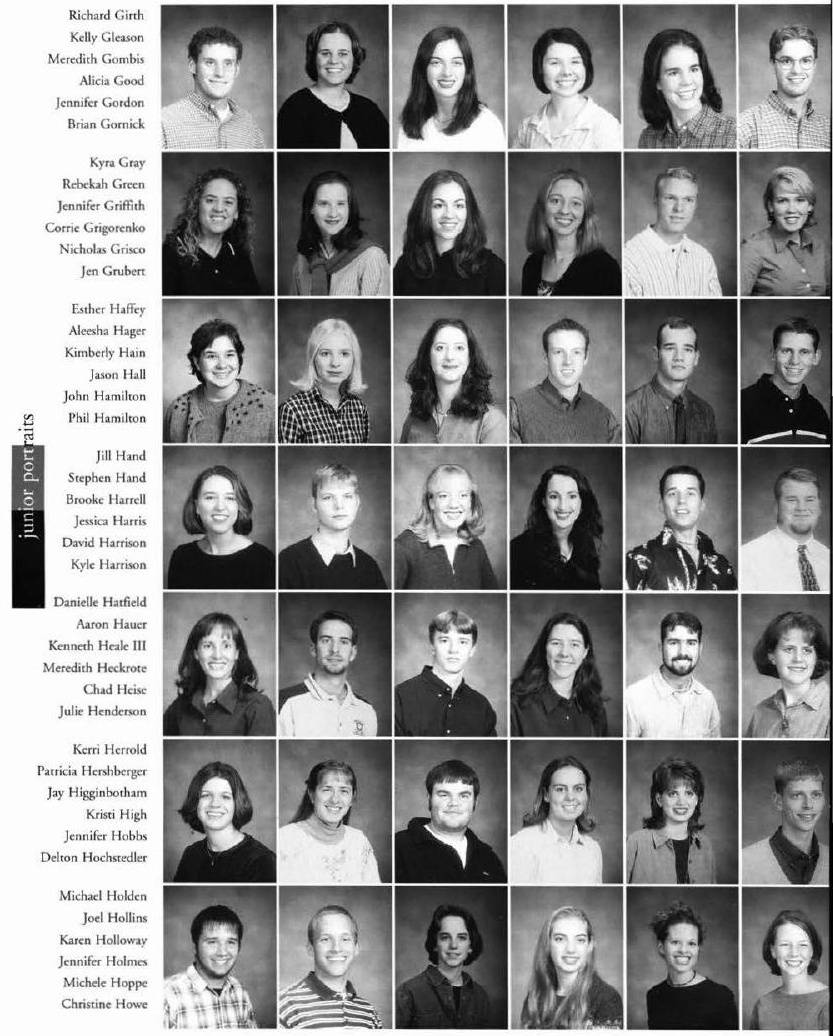




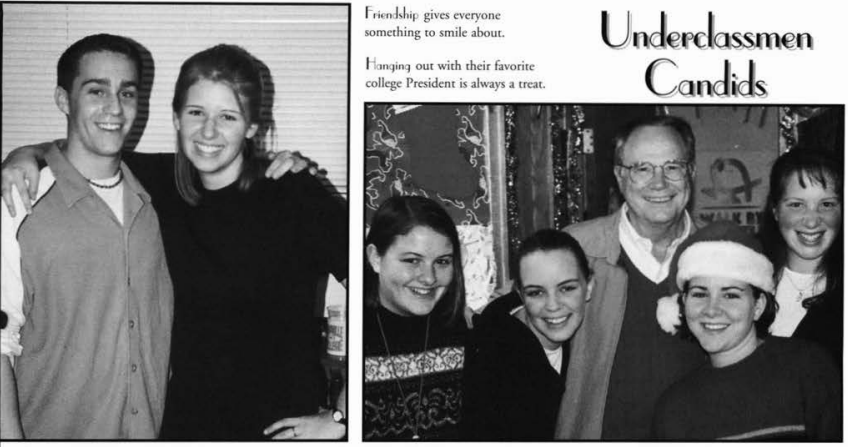

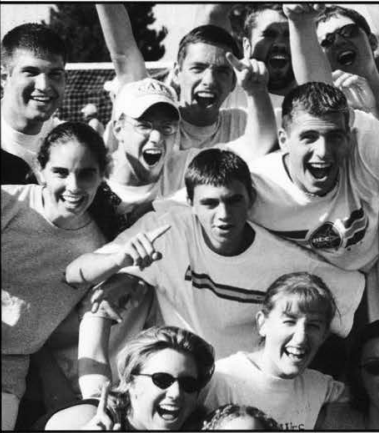

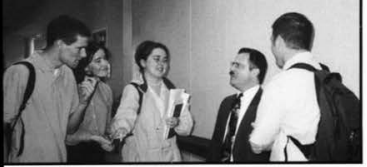

Wild and craxy is the norm

during the opening weekends.

A tea party in Willets Hall makes for a great study break.
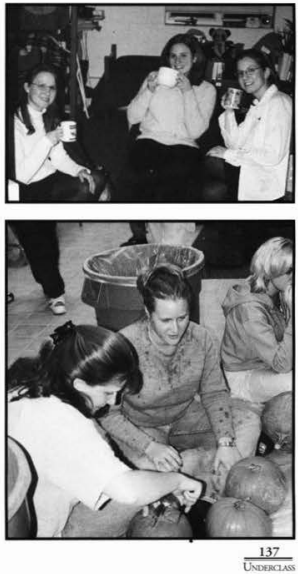
Chris Hughes

Allison Hume

Samuel Hunt

Jodi Hurlow

Micah Hutchins

Chris Hutchinson

Jennifer Ingersoll

Weston Issler

Andrew Jarvis

Margaret Jenista

Will Jenks

Matthew Johnson

Callic Jones

Lindsay Kaiser

Benjamin Kaminsky

Sarah Kanzeg

Ben Keener

$\cdot \frac{\mathscr{3}}{7}$

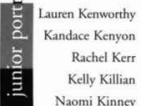

Heidi Kinniburgh

Erika Kipp

Cortney Kirkland

Bob Kocher

Katia Kocherova

Katic Koeppen

Lynn Koetsier

Rebekah Kozlowski

Jennifer Kranenburg

Brent Krumdiack

Stephanie Kurowicki

Seth Lacy

Kristi Lanz

Jennifer Larson

Nathan Lasso

Thomas Lawson

Jonathan Leach

Erin Lee

Karen Leininger
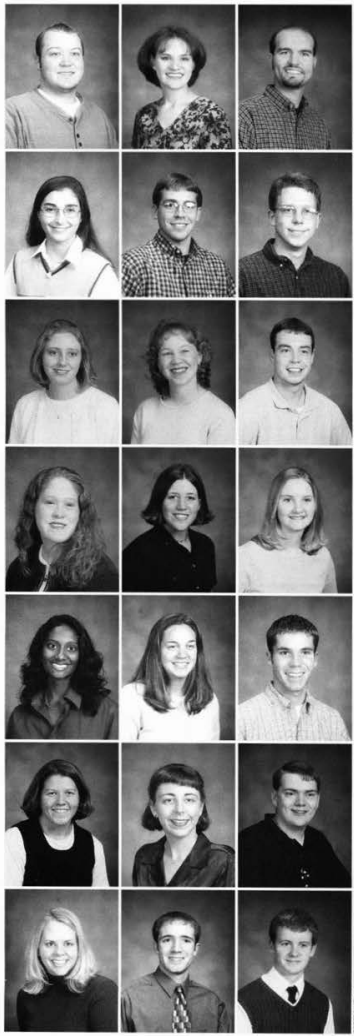
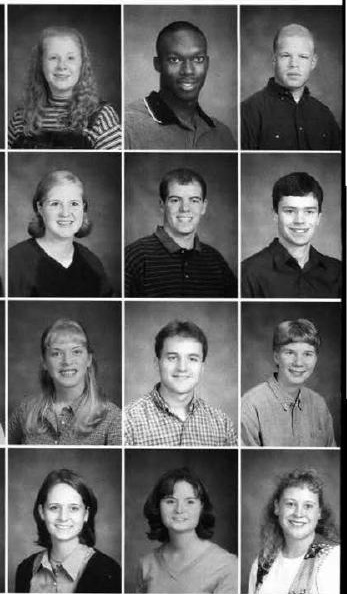

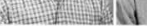
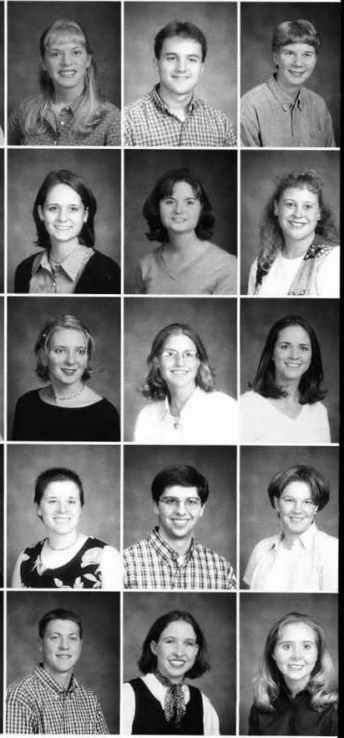


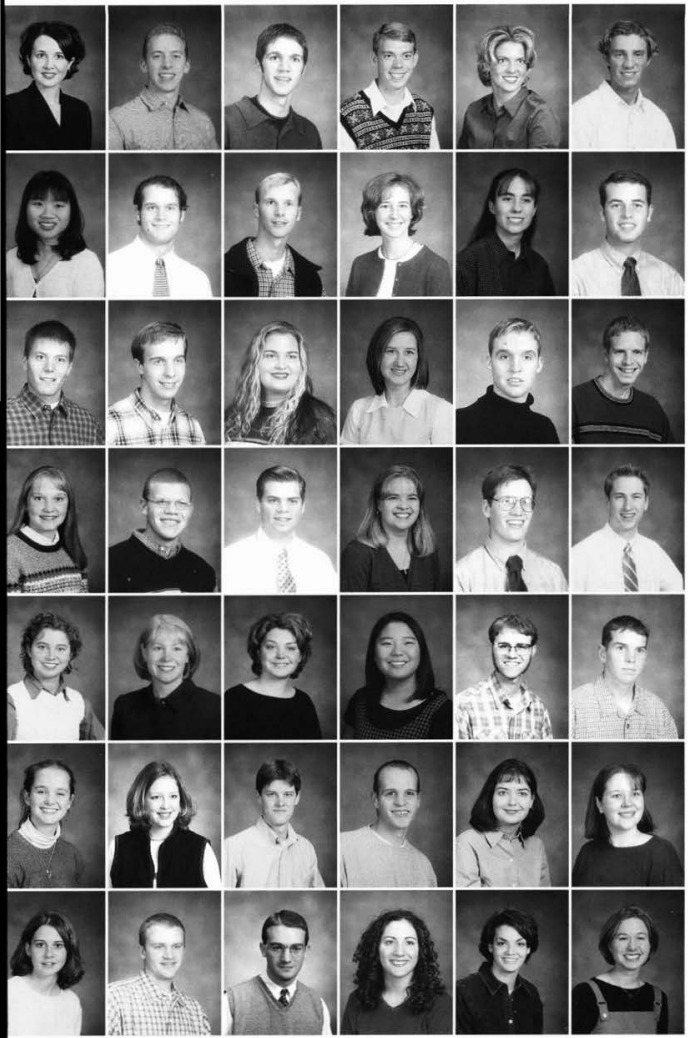

Sarah Leisure

William Leukhardt

Kevin Leverson

Nelson Lewis

Andrea Linamen

Samucl Logan

Esther Loh

Michad Lowstetter

John Lucas

Courtney Ludema

Jennifer Lutes

Robert Lutz

David Malwirz

Donald Manchester

Rebekah Mann

Katie Mansfield

Paul Manson

Joel Manwarren

Jessica Marks

Ryan Marks

Marthew Marshall

Wendy Martens

Matthew Martin

Serh Martin

Jennifer Mass

Jessica Mathias

Janice Maurizi

Jenna McCallister

Daniel McCoy

Phil MeCubbin

Erin McDaniel

Sarah McDonald

Ryan McGhee

Dave McKenzie

Elizabeth McKinney

Andrea McNeal

Deborah Meizzler

Andrew Mellish

Aaron Mercer

Jenna Metthe

Maren Meyers

Haley Miller 


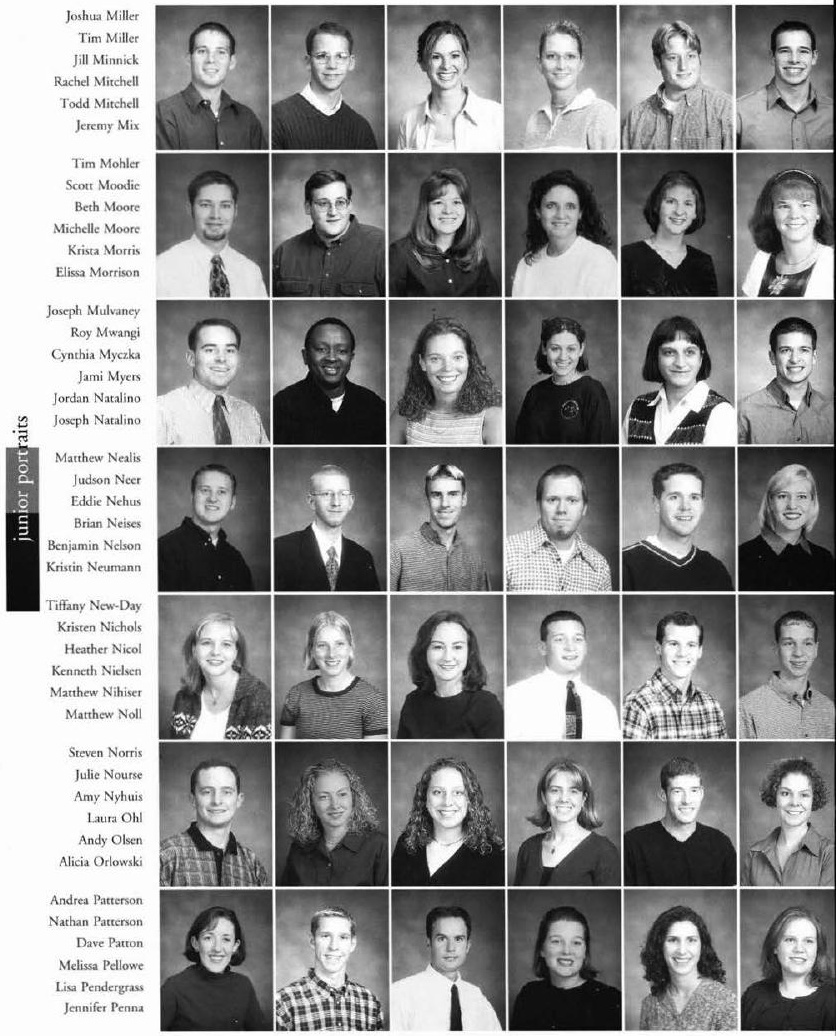




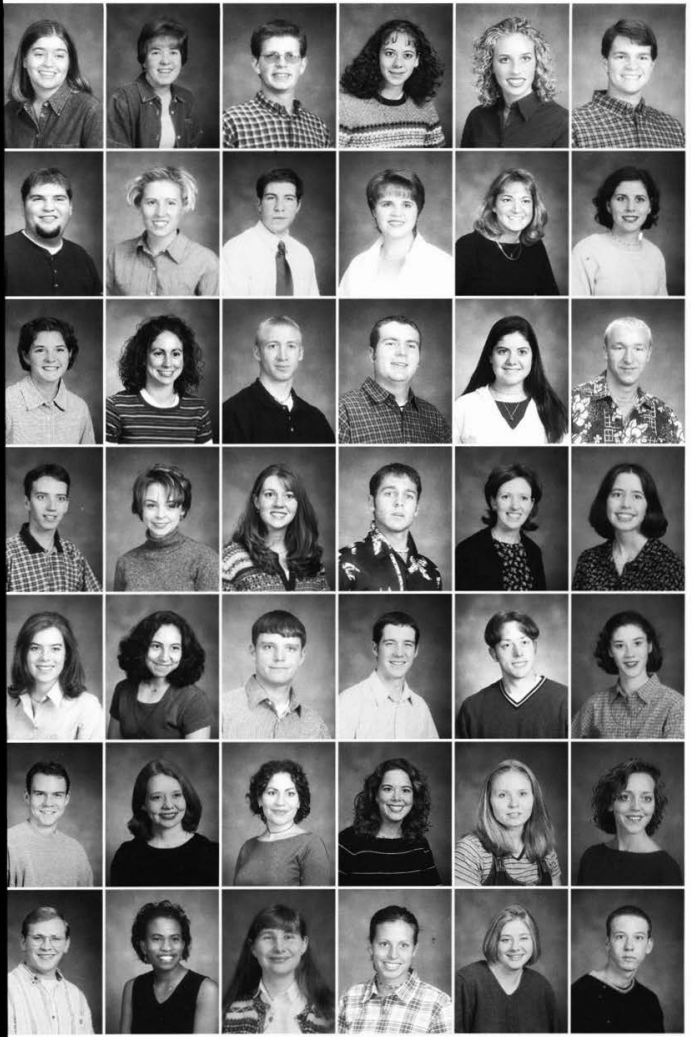

Melissa Pennell Janene Pereira

Drew Peters

LaChelle Peters

Lani Peterson

Spencer Phelps

Chris Phillips

Amber Philyaw

Jeremy Pierre

Jennifer Ploeg

Amic Poclman

Lindsay Pohlman

Sarah Pollock

Amanda Porter

Dan Potter

Nicholas Powell

Shelly Prado

Dave Prahl

Andrew Priano

Ruth Price

Erin Proulx.

Tim Prusha

Rebekah Pugno

Amy Reno

Molly Rexford

Melissa Reyes

Clifford Reynolds

Zach Rhodes

Dan Rickett

Charisa Ripma

Christopher Risheill

Melissa Robin

Joanna Robinson

Janelle Rock

Roberta Roc

Melissa Roesch

Tim Ropp

Sherri Ross

Tabitha Rowe

Annette Ruba

Amber Rumphol

Alan Sage 


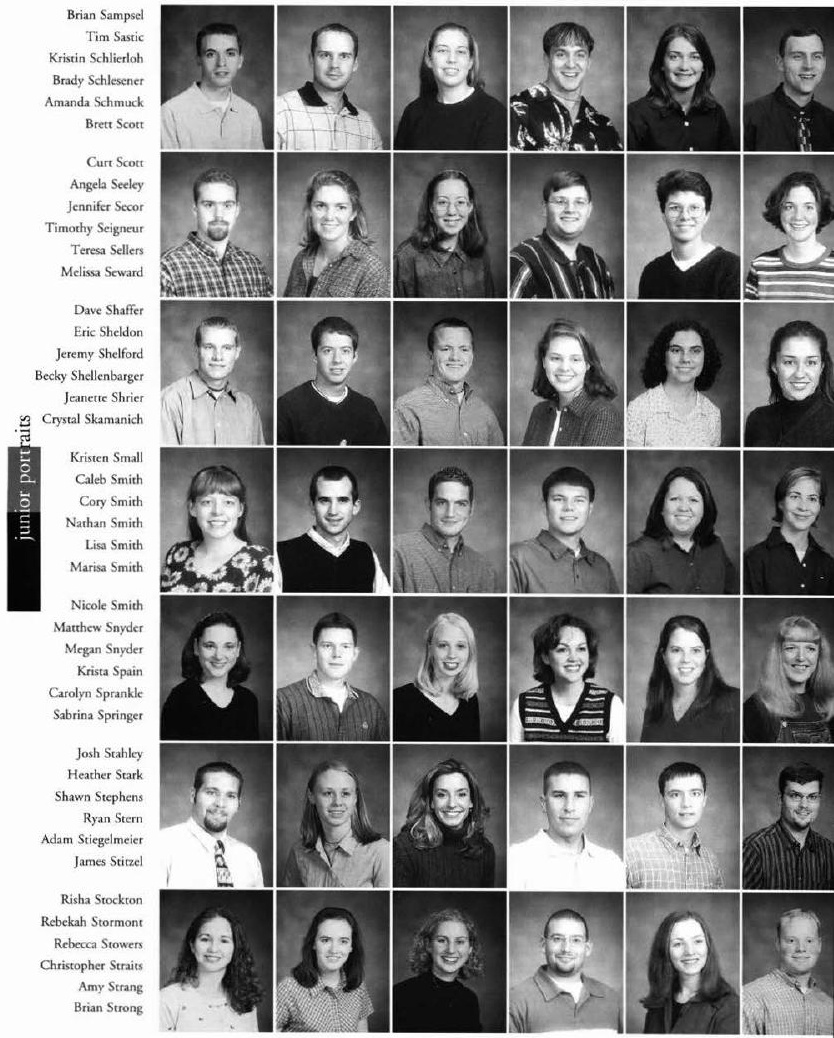




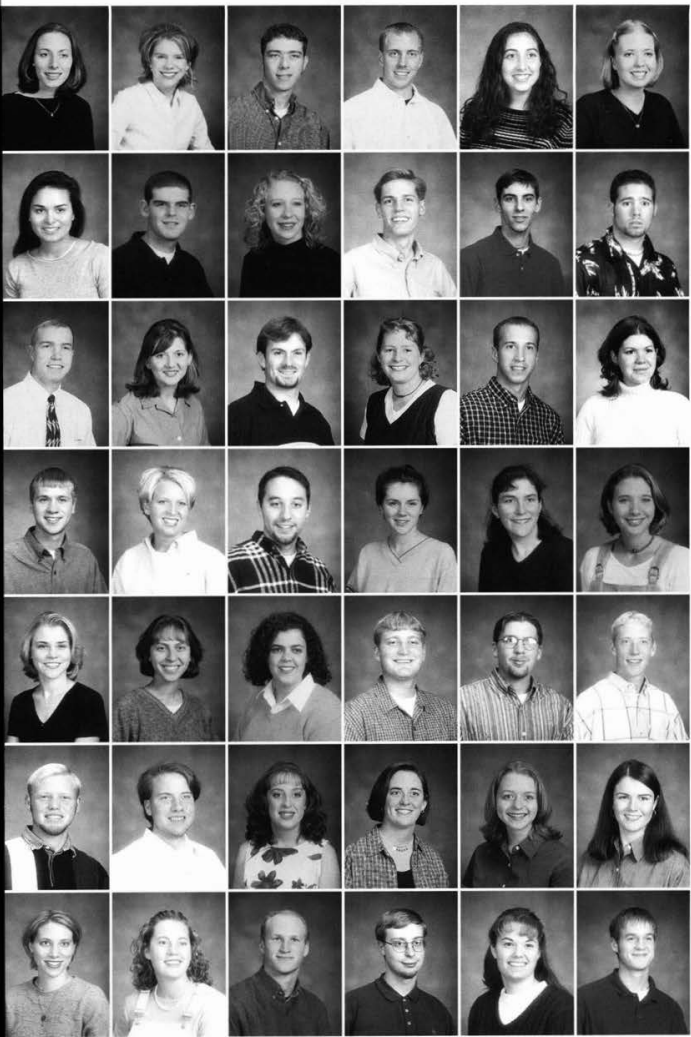

Dawn Struz

Jill Strychalski

Derek Summers

Tim Sutton

Juliene Swota

Heather Tallaksen

Tisha Tapp

Phillip Tate

Amanda Taylor

Joel Taylor

Patrick Thaman

Michael Tharp

Micah Thirey

Rebekah Thompson

Philip Thorsen

Sara Tomkinson

Russell Toms

Jennifer Tong

Joshua Tromp

Jennifer Troyer

Nick Tucholski

Amber Tucker

Lori Turner

Kelly Tyson

Jenni Urban

Erin Vallowe

Rebecca Vitarelli

Ryan Vogel

Benjamin Vroman

Steven Wakefield

Kenneth Waldock

William Walker

Alison Warren

Beth Weaver

Jennifer Weaver

Andrea Weber

Stacie Weinert

Kristil Wendler

Eric Wentz

Nathanael Weygand

Christina Wheatley

Ben Whipple 


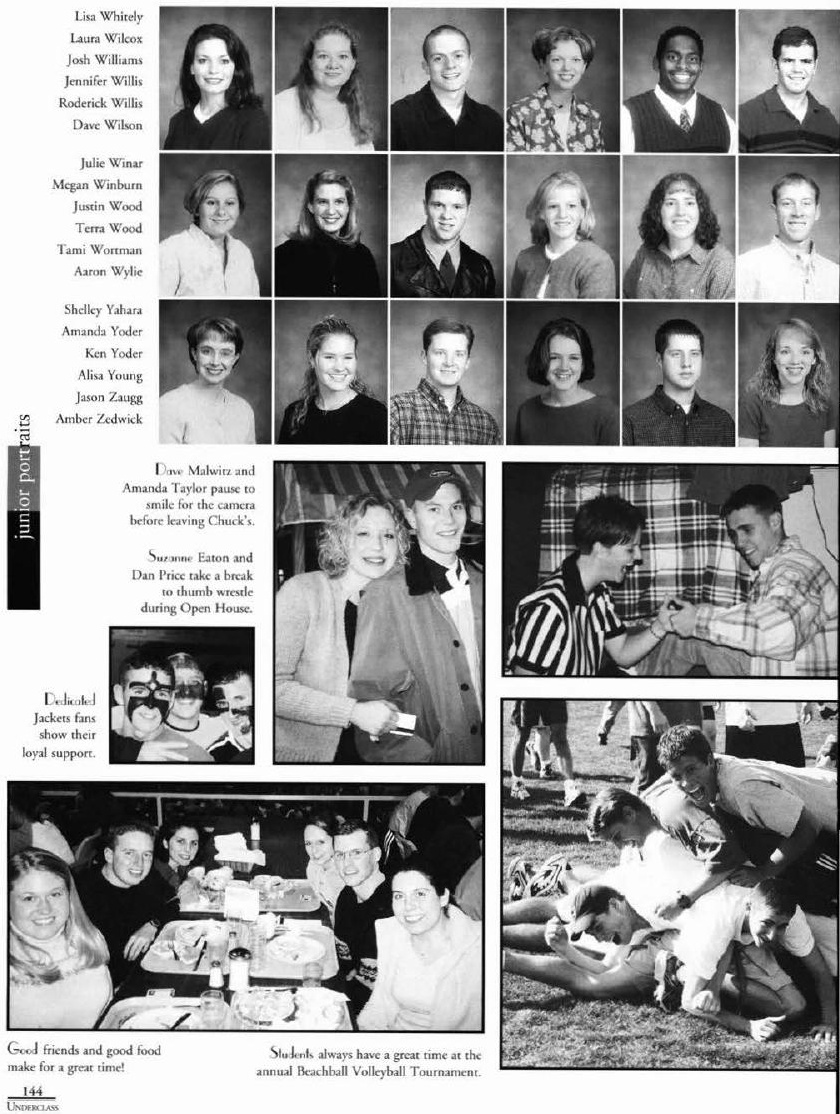




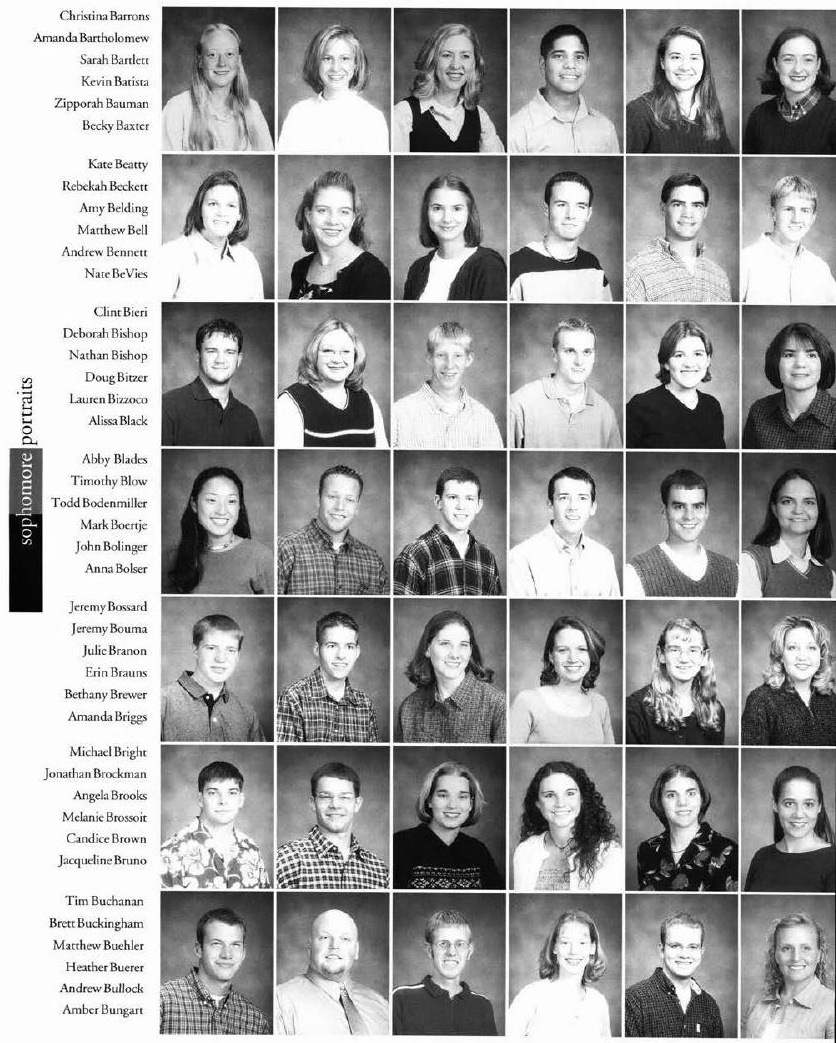




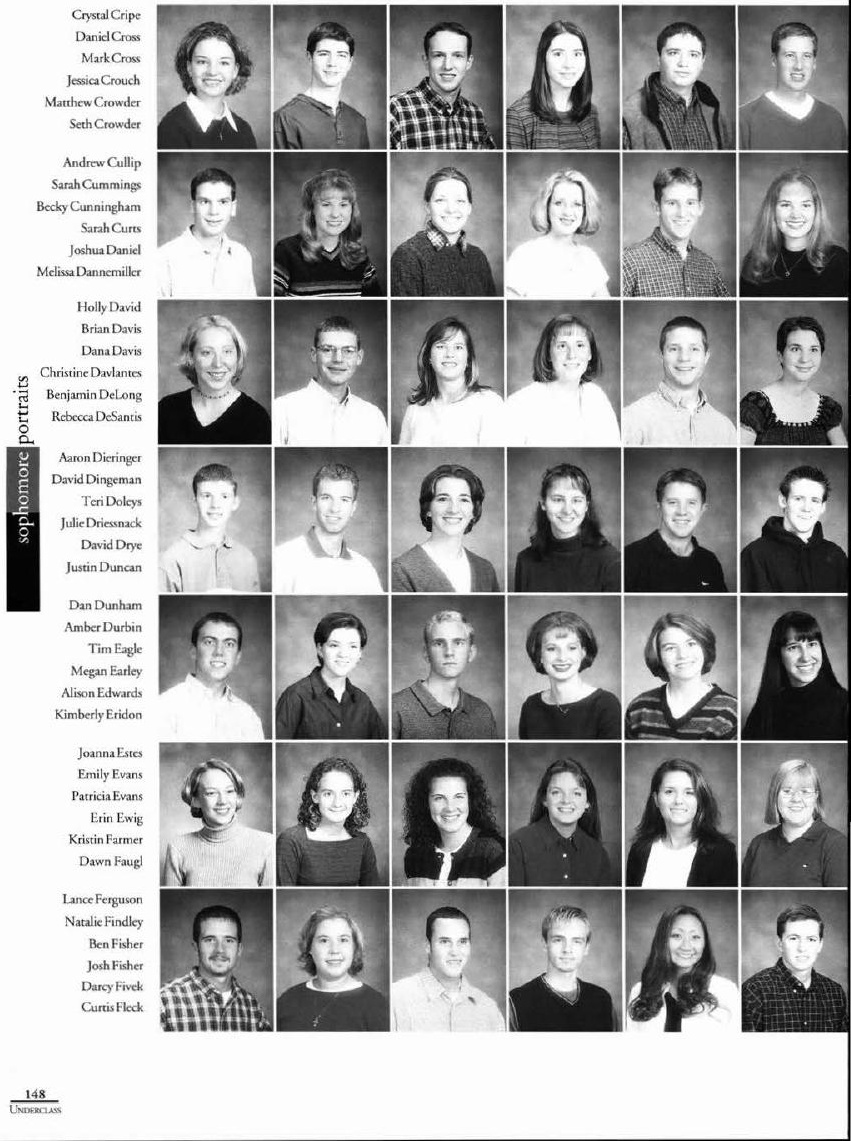




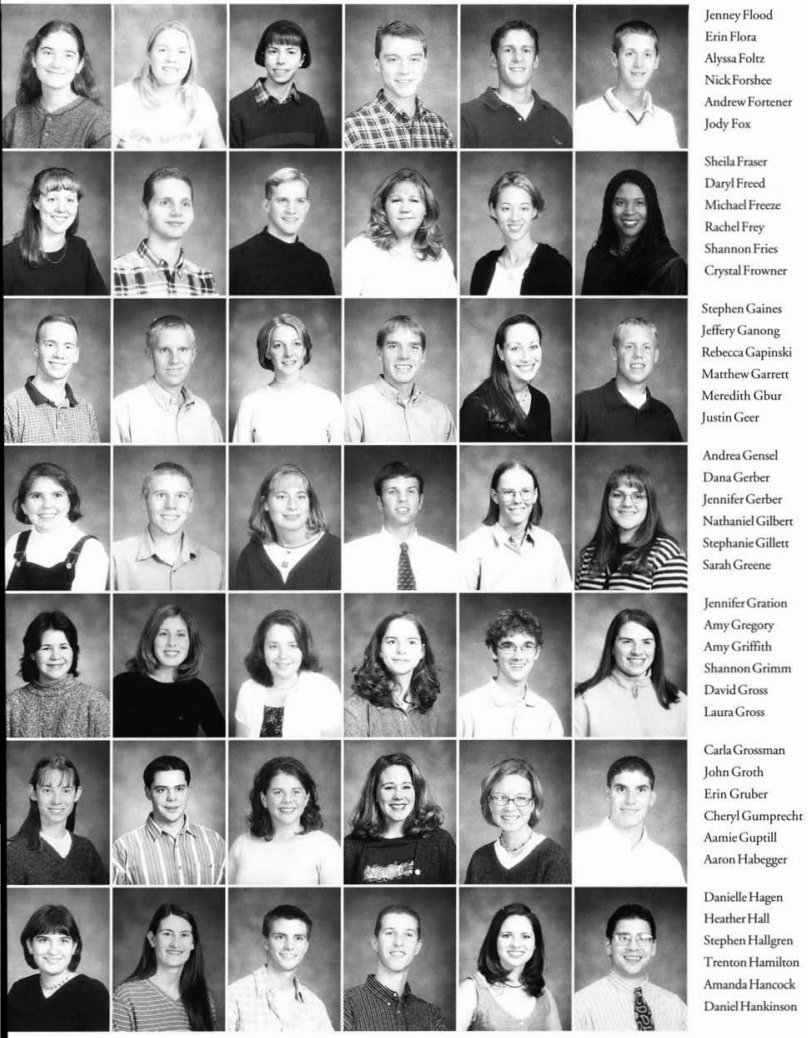




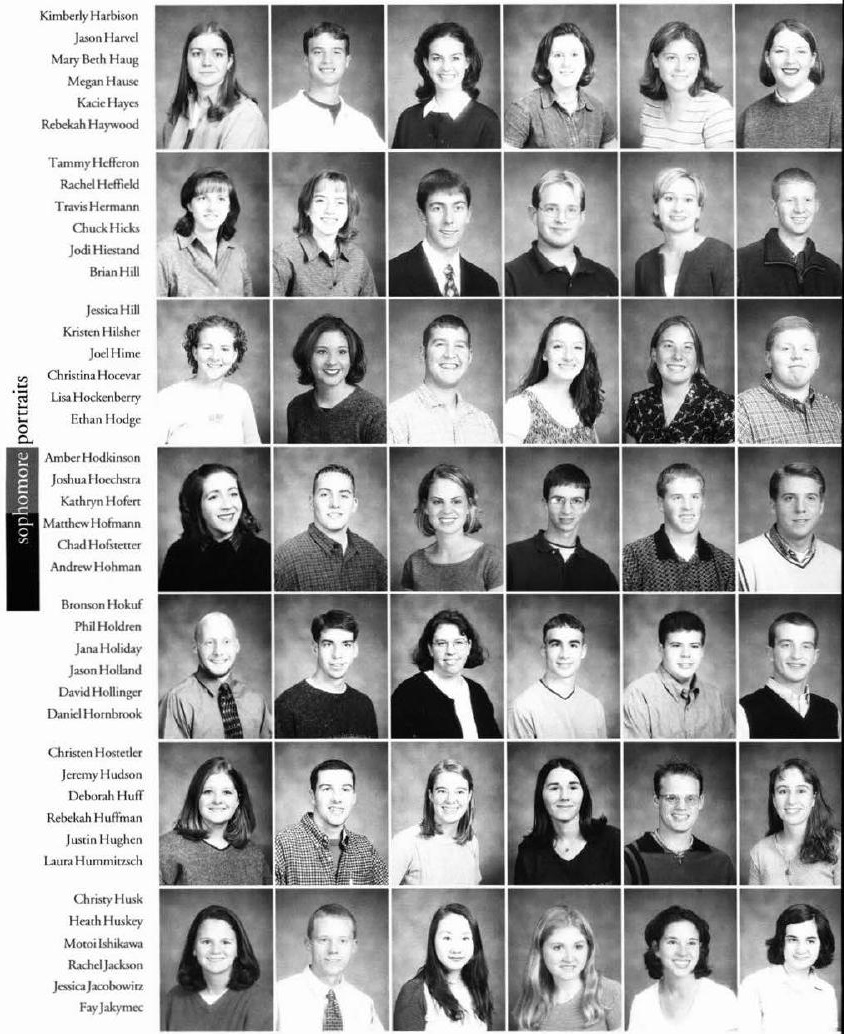




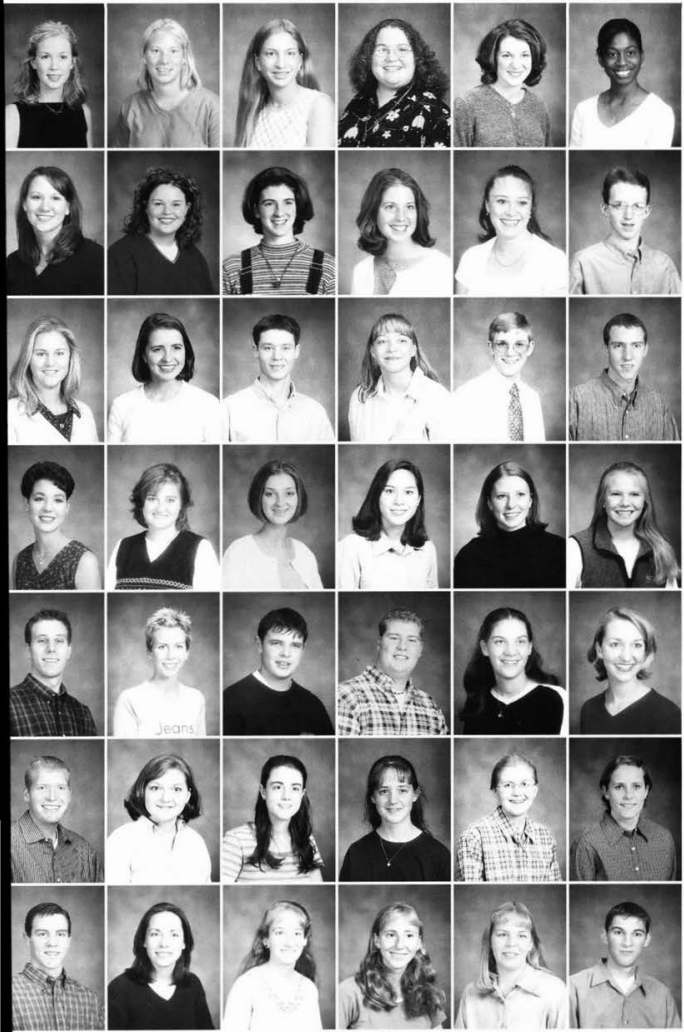

Joy Jennings

Rebeccalensen

Kristina Jonker

Kalcyjohnson

Meliss Jones

Wensa Juleus

JessicaKaiser

Kelly Kandel

Bethany Keilman

Esther Kelly

Derika Keenan

Steven Kenny

Katie Kepner

Kristen Kerr

James Kessler

Kelli Kessler

Nathan Kibelbek

Jonathan Kirby

Lauren Kirby

Elisabeth Klawer

Kristen Kline

Linda Knauer

Melissa Knaus

Karen Knudson

Timothy Kopp

Karin Kokeny

Brad Kormash

Jared Koverman

Debbie Krick

Holly Kuhn

Ryan Kurzen

Kelly Lambert

MclissaLasso

Lisa Lauritzen

Veronica Lawton

Sara-Beth LeBlanc

ThomasLee

Katherine Lemman

Hannah Livingston

Katie Livingston

TracyLoudermilk

JamesLove 


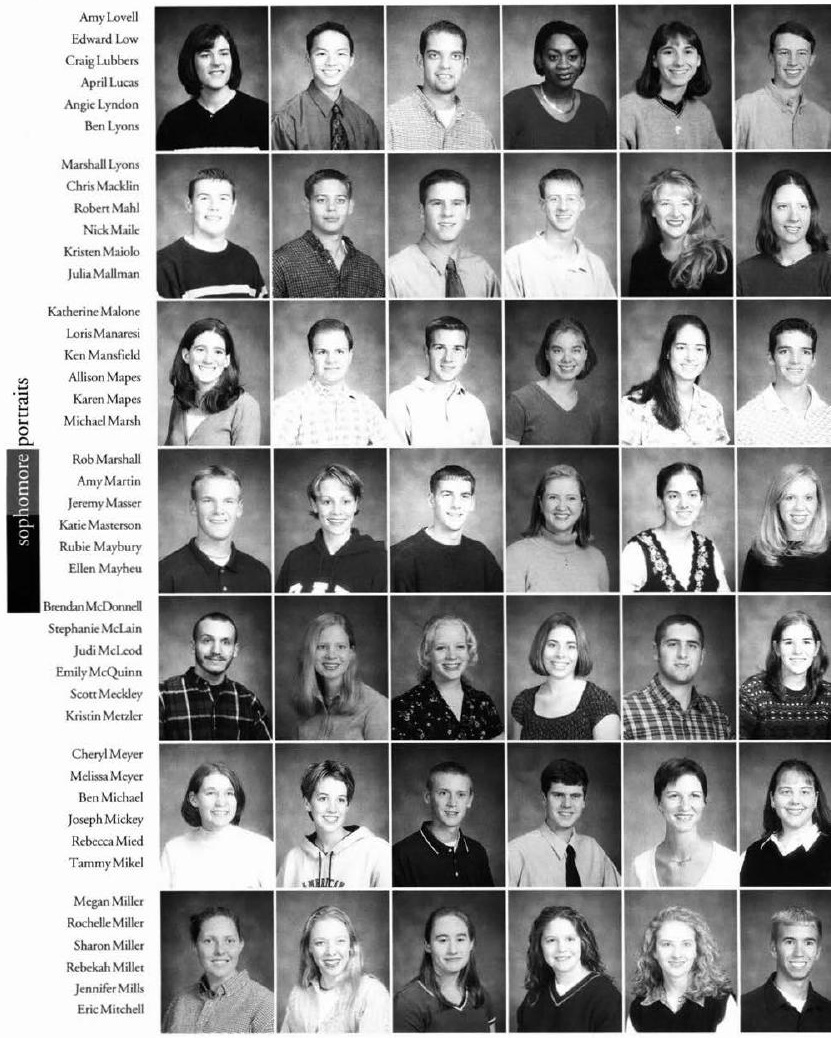


Amanda Pilgrim

JillPinney

Robyn Pitman

Daniel William Plaatje

Mike Poelzer

Christopher Pollard

Sarah Poscgate Josh Postlewait

Matthew Powell

Timothy Priano

Daniel Price

Laura Price

Michad Provencher

HeatherPursicy

Jacob Pyles

Alicia Rambikur

Sarah Raszcja

Robyn Rausch

.

$\frac{\frac{2}{6}}{\frac{2}{8}}$

Gooffrcy Redkternwald

Diamond Redmond

Alison Reemtsma

Rebckah Reynolds

David Rheam

MatthewRichmond

Julia Roberts

Karen Robinson

Daniclle Rogers

Benjamin Rosner

Sarah Ross

Aaron Roth

Gregory Roth

Michelle Ruhlman

Greg Sanderson

Benjamin Saturley

Francis Saul

Kurt Savage

RebeccaSawyer

LeslieScadding

Grayden Schafer

KimberlySchaffer

JessicaSchipp

NickSchlappi
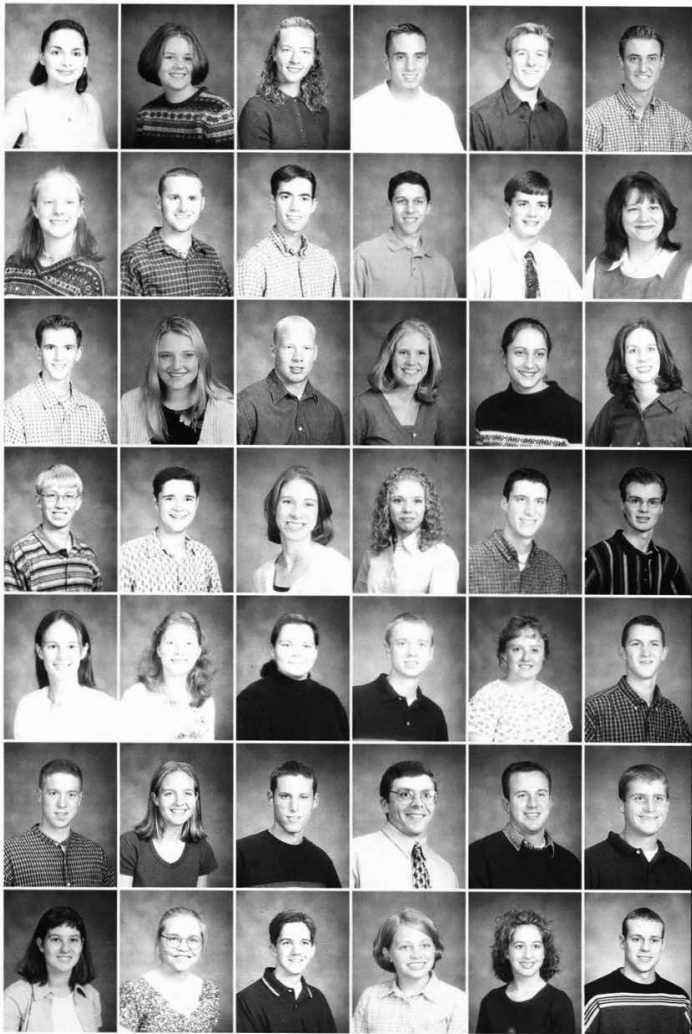
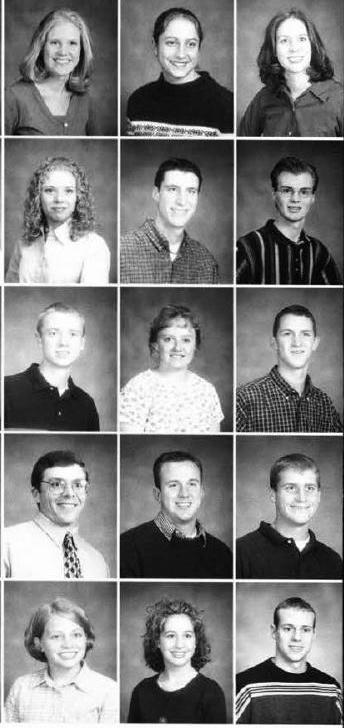

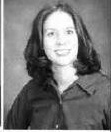




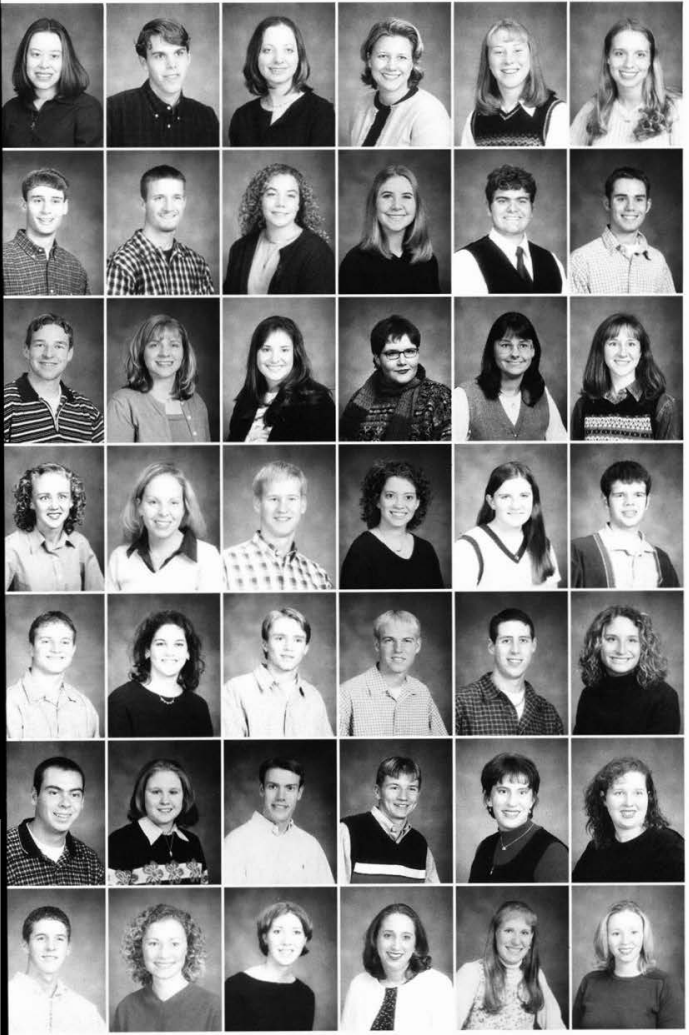

JessicaSchmidt

CalcbSchnake

Alison Schneider

KateSchriemer

Kristin Schutter

Amber Scott

David Scals

Joel Shank

StaceyShcolnick

TammiShcldon

WillyShelton

PhilipSheward

CharlieShifflett

MeredithShinko

Heather Shoemaker

Frances Shrubsole

Joy Marie Shustack

Lisa Simpson

Nicole Sinzinger

SarahSkillings

Benjamin Skurdal

MelanieSlabaugh

ErinSmith

JeremySmith

JasonSmith

Misty Smith

ScotrSmith

Brian Snyder

RobSnyder

Wendy Somers

John Sorrell

EmilySpangler

Curtis Sparks

Pete Springirth

HeidiSproul

Elizabeth Spruill

AaronStatler

Jennifer Steely

Elizabeth Steenwyk

KelsieStciner

KatherineSteingass

AmberStenger 
Carla Stoltenberg

StephanieStrong

Joseph Strychalski

DaniclSudlow

Craig Sullivan

DonnaSwan

Jonathan Swanson

Laurie Tait

EricaTaldo

Bethany Tate

Jeremy Tencza

Jessica Terry

Rebekah Teusink

Rachel Thonpson

Christine Trago

Carl Traub

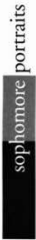

Mark Van Lenten

Rachel Van Loon

Rebecca Vandemark

Edward Vander Bush III

Tiffany VanHart

Michael Vanlier

Rachel Danielle Vink

Benjamin Vlug

Renac Vogr

Laura Volpe

Trisha Wabeke

Kent Wagner

Kimberly Wagner

Lynette Wagner

Ruth Wagner

Bethany Walden

James Waldo
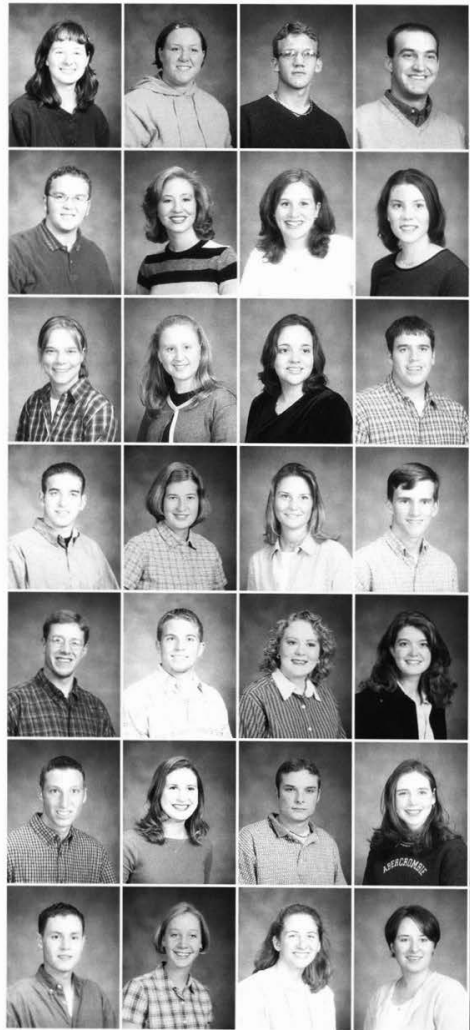
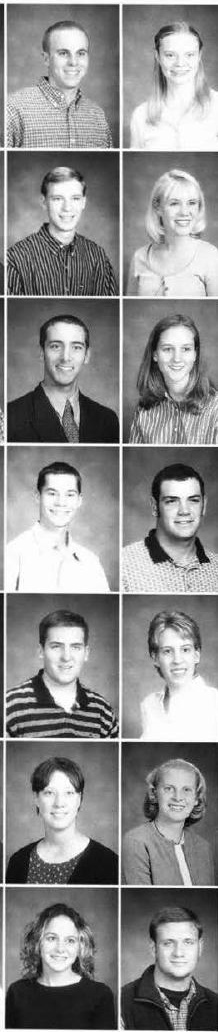


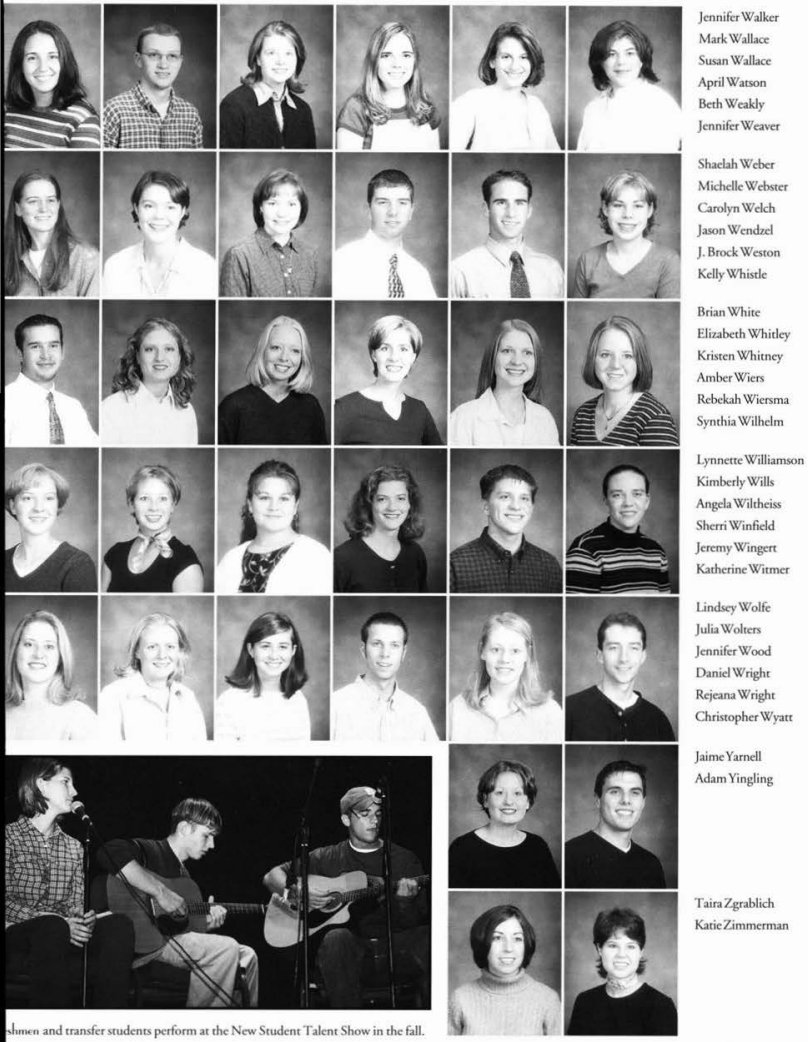




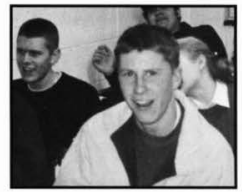

Jeel Estes enjoys being surrounded by friends.
A field trip to Old Man's Cave is a good study break.
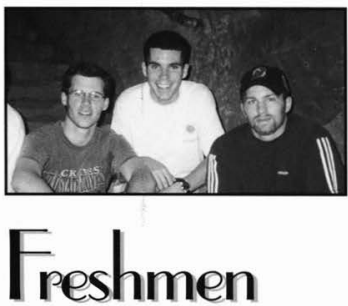

Chesler Wakefield prepares to run.

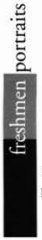

Kolawole Adeoluwa Heather Albernson

Brenda Allen

Nicole Allen

Scort Allen

Nicole Ambrosius

Summer Allison

Desiree Anderson

Jennifer Apple

Sarah Arnstein

Christina Babcock

Eric Babson

Ryan Baitaglia

Anelise Baits

Krista Baket

Leah Baker

Sarah Baker

Jessica Baratta

Amy Barnette

Adam Bartlett
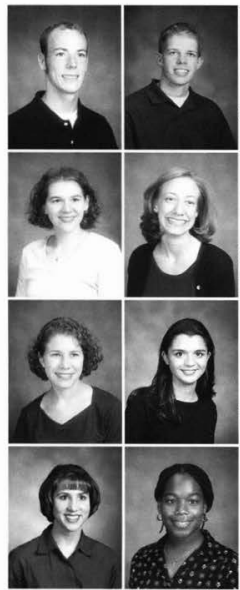

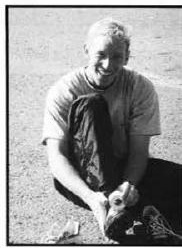

Class of 2003
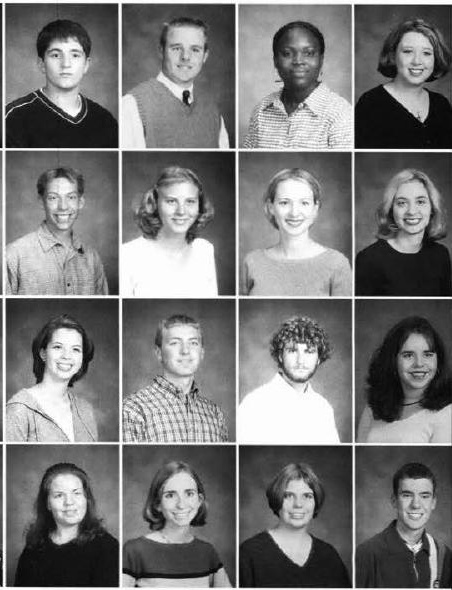


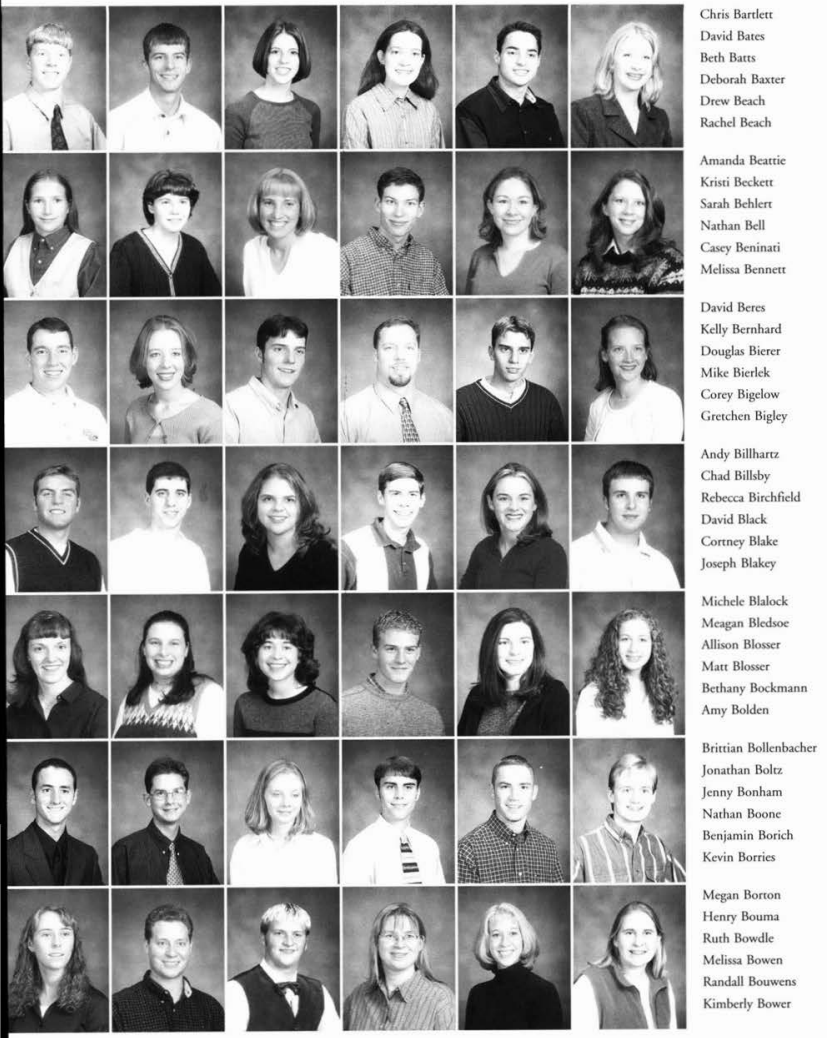




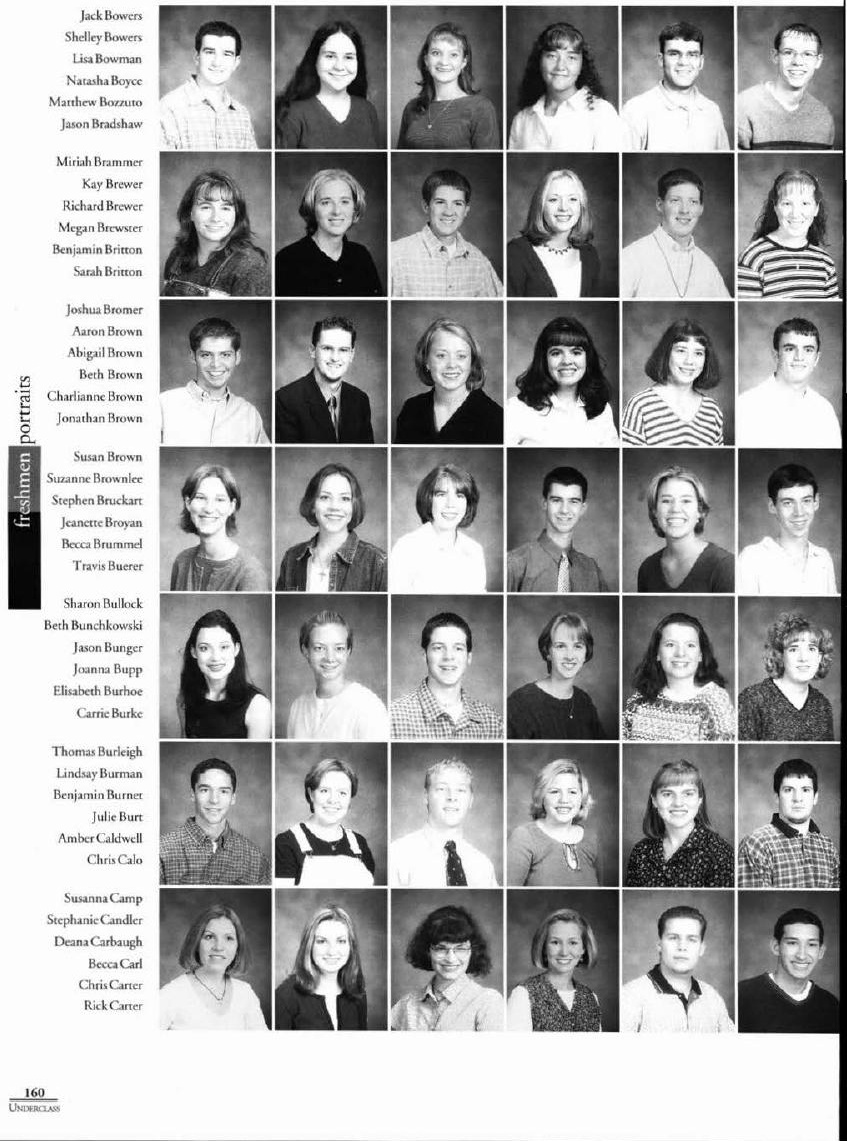




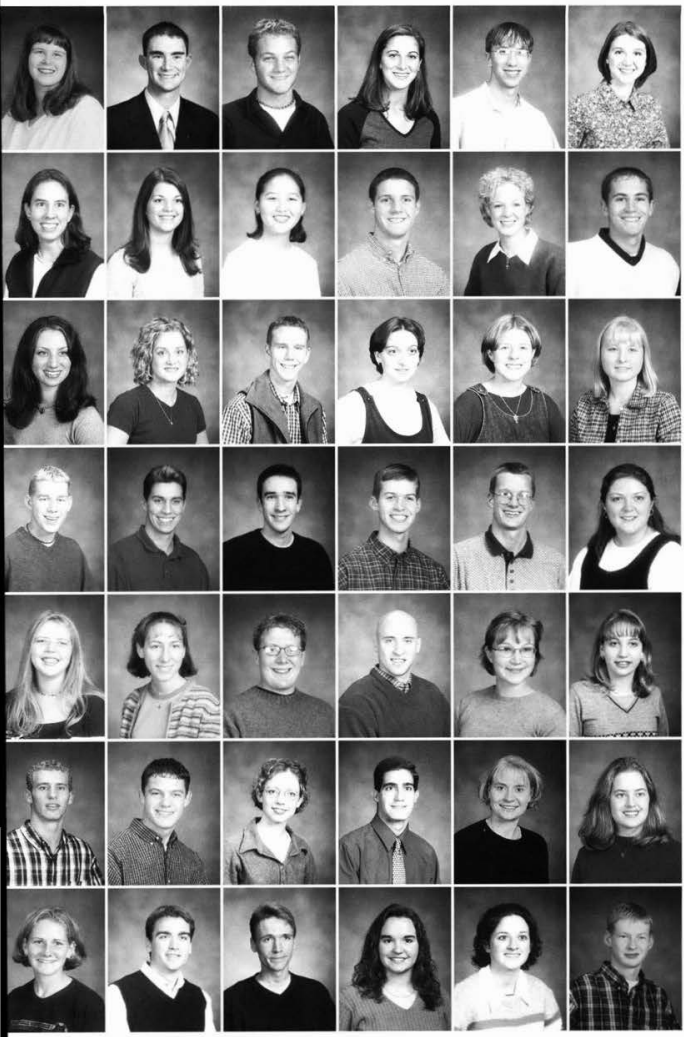

Lori Carveth

Travis Casper

Jonathan Chaney

Erin Chester

Robert Chestnut

Geny Chipe

JenChmiclewski

Julia Christner

JenniferCicchini

Thomas Cizauskas

Kristen Claeys

Jon Clark

Casey Claypool

Alicia Cochrane

MichaelCoffey

Christina Colarossi

Debbie Compton

NicoleConn

Brian Coon

Charlic Corrick

Tim Cox

Adam Craig

Kevin Craig

Janelle Criner

Emily Cronbaugh

TheresaCrunelle

Ryan Culpepper

Jonathan Culver

LisaCulver

CaraCummins

Jeremy Cunningham

Trevor Cupples

Adria Curts

Michael Daiello

Sarah Daniels

Linda Dannemiller

Danielle Davidson

Jonathon Davies

Aaron Davis

Jennifer Davis

KaraDavis

Matthew Davis 
Karharine Dawkins

Becky Degarmo

JuliaDeHart

Ken Delaney

Kristen Derwiler

Chanelle DeWeese

Jake Dickinson

Jeff Diggle

Michacl Dishon

Abby Dixon

Erin Dooley

Kevin Dougherty

Alan Dow

Michelle Drumbeller

David Dudick

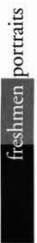

Amanda Dunn

Benjamin Dunn

JessicaDunning

Elissa Dvorak

Adam Dyer

Kari Eaves

Callie Edgington

Kimberly Edlund

Jen Edwards

Shac Elam

Tamara Ellington

CherylEllior

Jeremy Ellis

Jason Elwell

Gregory England

Amy Estes

Joel A. Estes

Joel D. Estes

Mary Evans

Andrew Everett

Heidi Everet

Bethany Farley

Jonathan Farrell

AnnaFarwell

Matthew Feldmore

Adam Felmlec

Kristin Felts
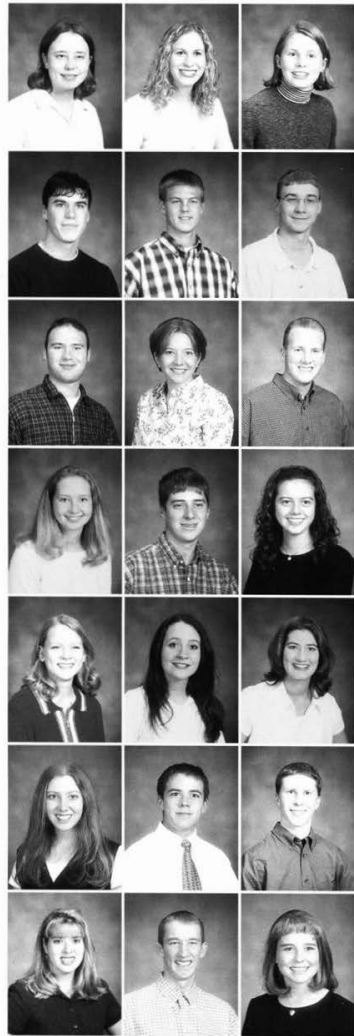
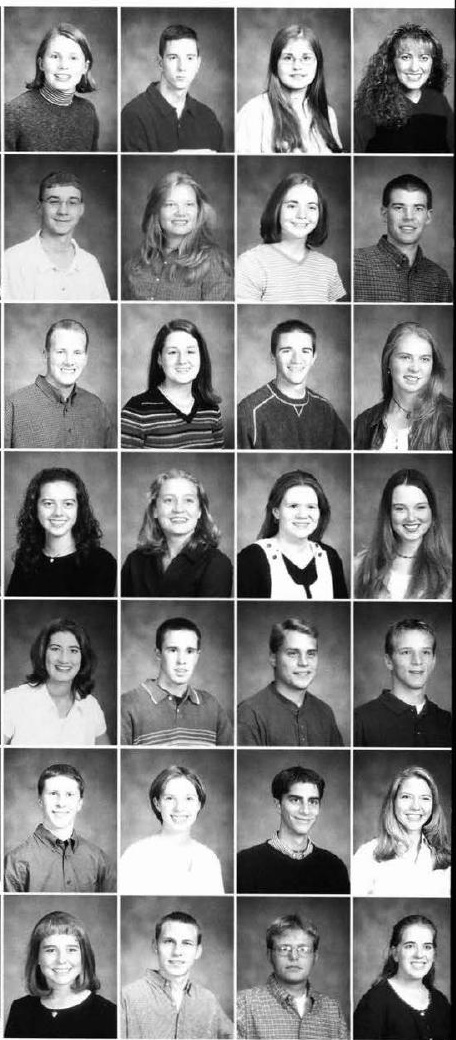


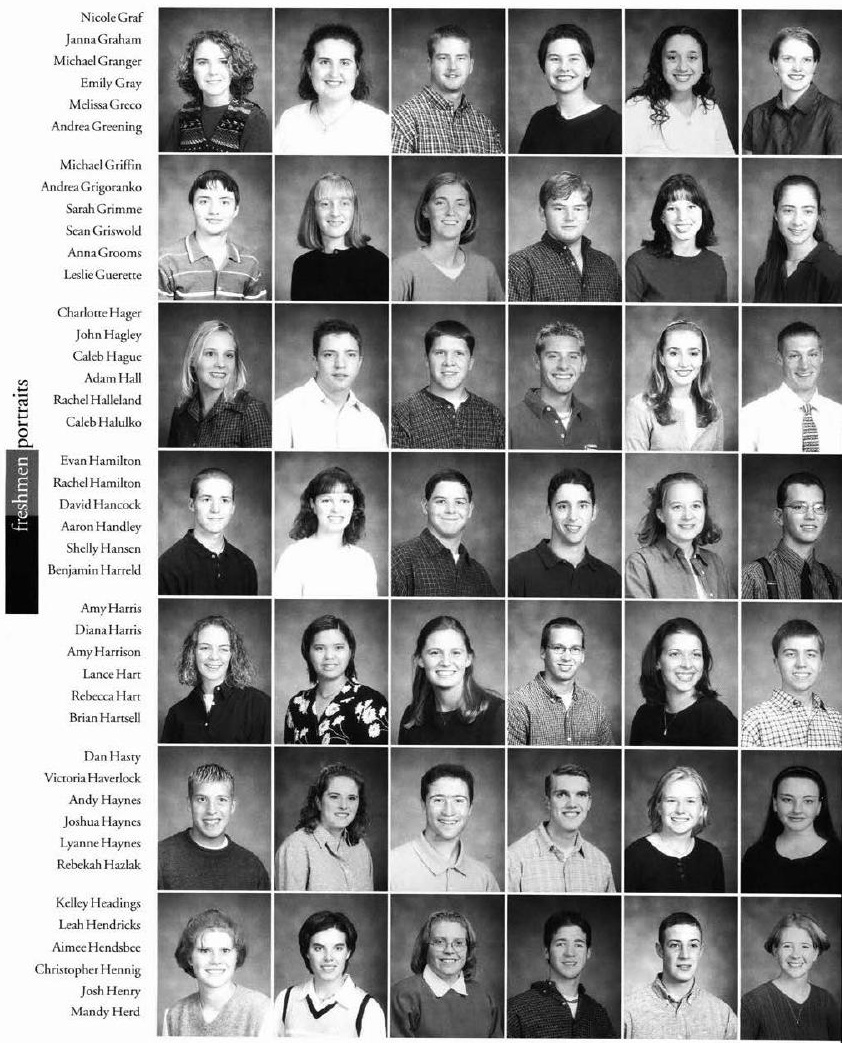




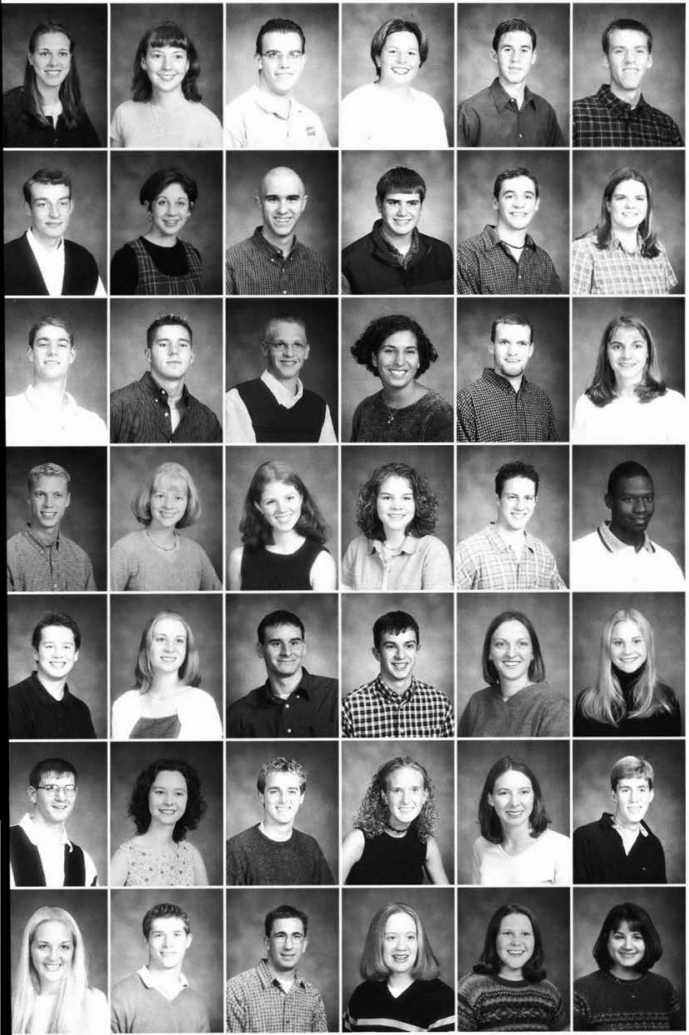

Christy Herman

Julia Hilbish

Michael Hill

Denaye Hilty

Peter Hochstaetter

Brandon Hoffman

Jason Hohertz

Sarah Hoke

Mark Holden

Jason Holmes

Aaron Hoober

ChristinaHooker

Jesse Hornback

Justin Horst

Justin Houck

LaraHourani

David Huber

Megan Hueni

Jonathan Hunsberger

Jessica Hunt

Alia Hunter

Alisha Huster

Brian Hunter

Samuel Hutchins

Joshua Hynes

Abby Iden

Chad Ireland

Nathan Irwin

Amber Isbell

Kelly Issakainen

Joel Jackson

Katic Jackson

MarkJaskilka

HeatherJenkins

DaniJenks

Ben Johnson

Jessica Johnson

Josh Johnson

Matthew Johnson

Tarajohnson

Whitney Johnson

Laura Jolly 


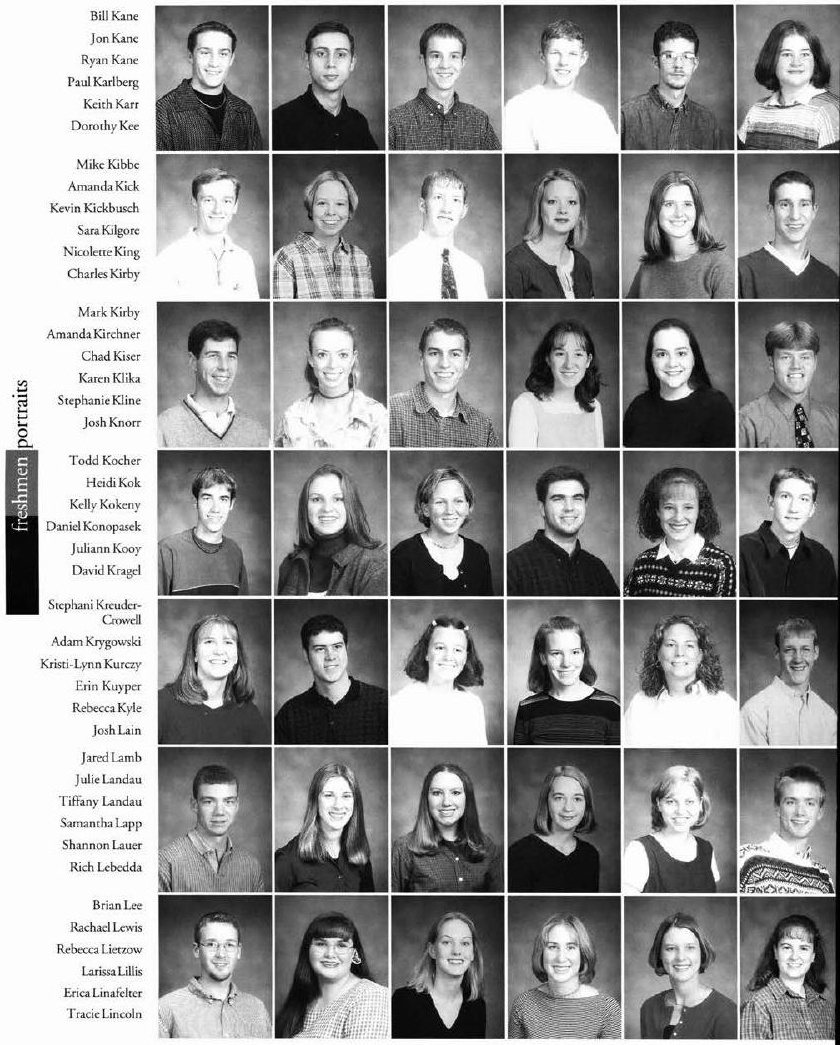




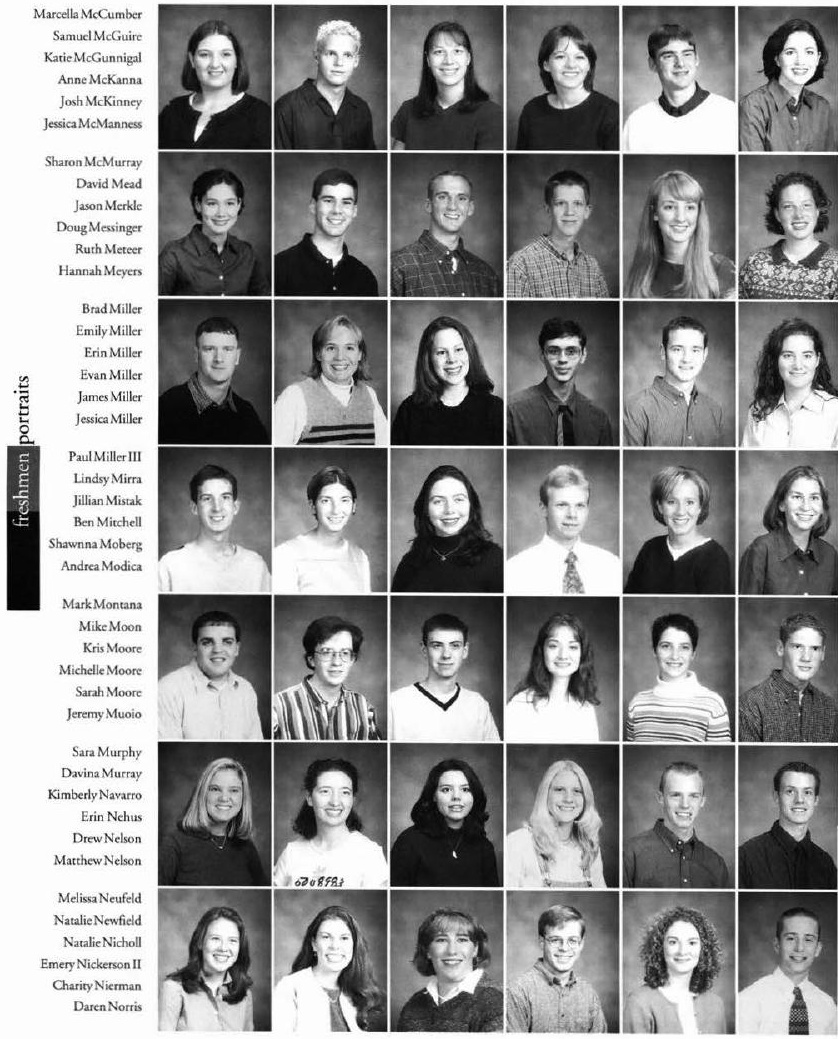




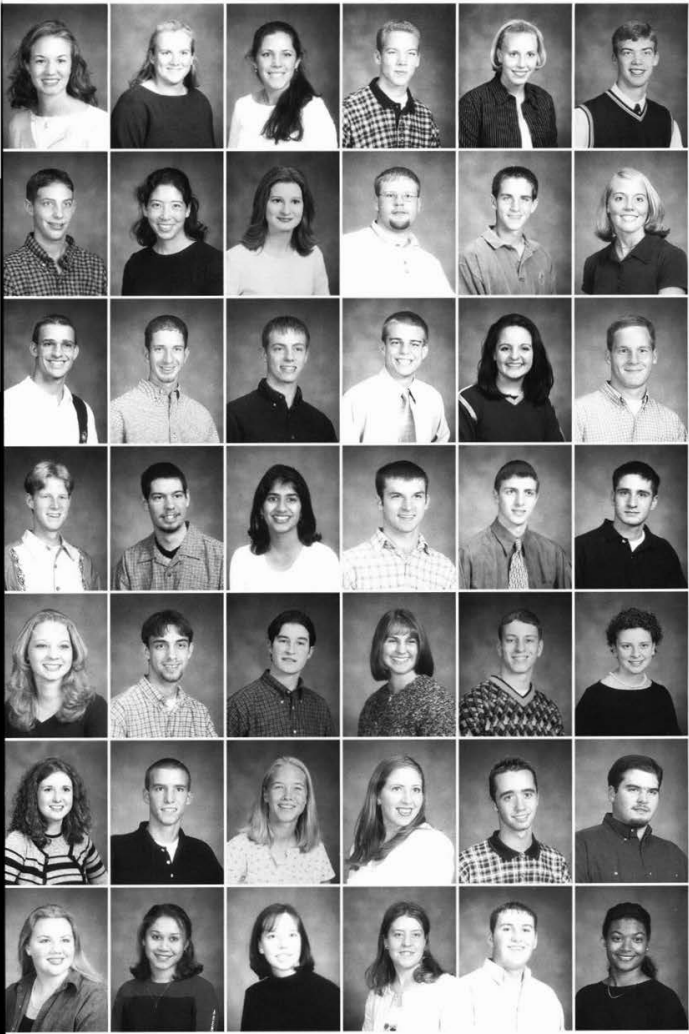

Christina Oberdove

Karin O'Connell

Kelly O'Connell

Justin Ohlinger

Sarah Oleszczuk

Lars Olson

Aaron Osterman

Molly Otsuj

Teresa Ott

Jeremy Page

Stephen Palmer

Stephanie Part

Joshua Patterson

Jeremy Patton

Gene Peelman

Todd Pellowe

Deborah Perez

Chancy Persons

Keith Peters

Mark Peterson

Jyoti Philip

Eric Phillips

Tim Phipps

Jessup Pierce

Kristin Pierce

Russell Pierpont

Daniel Pierre

Megan Potter

Joshua Paulick

Katic Powalski

Ericka Powell

Philip Preston

Cindy Probus

Elisabeth Pugno

Victor Puhy

Kelly Purnmell

Julie Quinn

Stephanie Rankin

Staccy Rasmussen

Alice Rau

Andrew Raymond

Brionna Raynor 


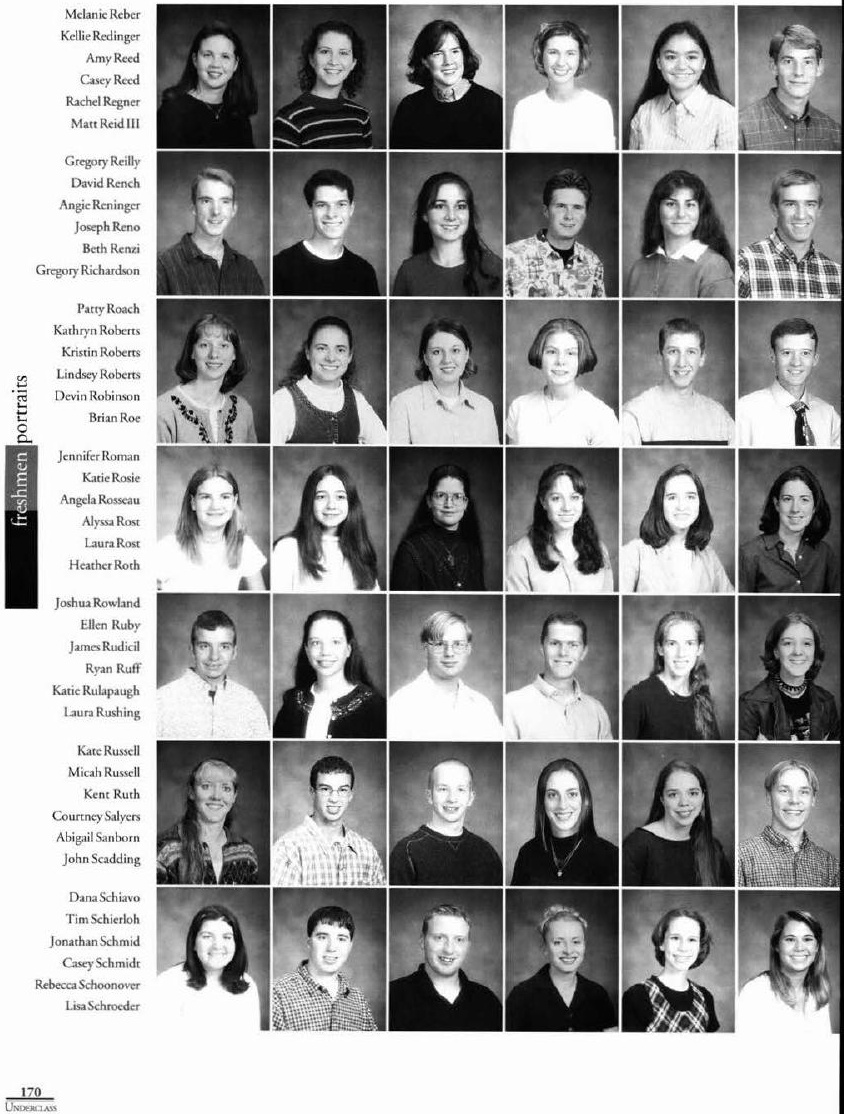




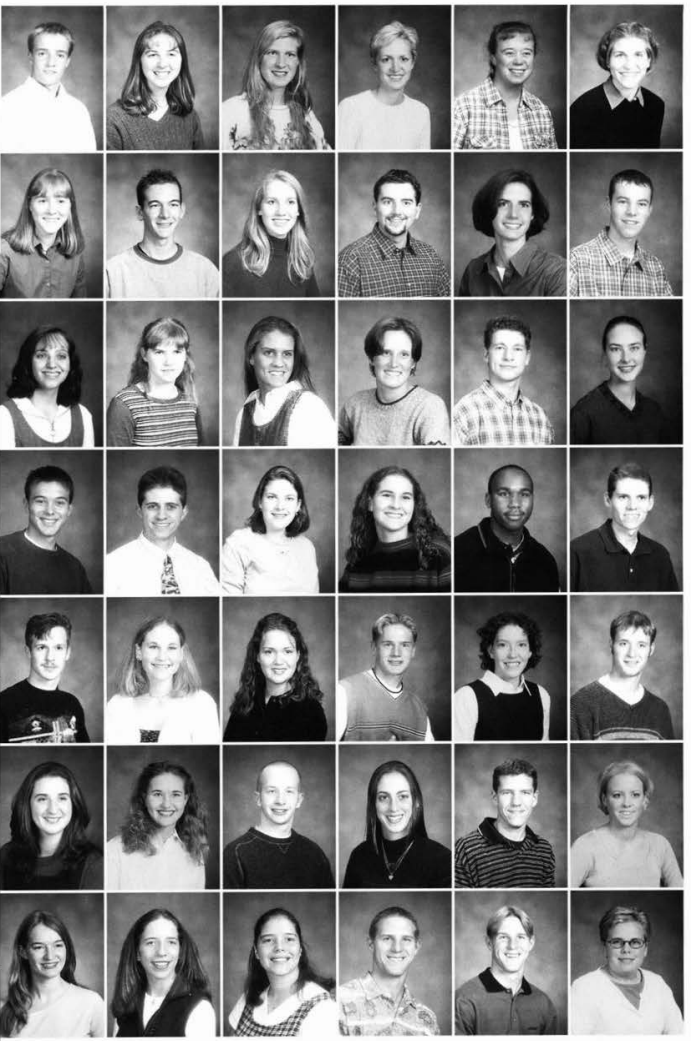

Matthew Schroeder Rachel Schuh Liz Schumacher Christina Schut Lisa Scott

Beth Seachrist

LeeAnn Senseney Chris Seymour

Katic Shank

Ryan Sharp

Sarah Sharp

Dana Shaver

Tara Sheesley

Lisa Sheldon

Janelle Shev

Celia Shortt

Matthew Shuman

Allison Siddall

Jason Siemer

Andrew Smith

Carissa Smith

Jennifer Smith

Matthew Smith

Matthew L. Smith

Matthew W. Smith, Jr.

Meagan Smith

Jessica Snedeker

Dan Spanagel

Mindi Sparks

Timothy Sparks

Charity Spencer

Catherine Spink

Brad Spitzer

Abby Stafford

Andrew Stafford

Amy Standish

Ariel Starbuck

Tiffani Stats

Melissa Stauffer

William Stephens

Wes Stephens

Amanda Stevens 


\section{JessicaStevens \\ Seth Stevens \\ Edward Stewart \\ Rebekah Stewart \\ David Stiegelmeier \\ Christopher Stokes}

\section{Jesse Stone \\ Santino Stoner \\ James Strasler \\ Nisha Strauch \\ MarkStrickler \\ Brandon Stuckey}

PatriciaSutter

Jason Swan

Garret Swartzentruber

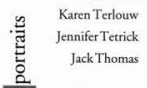

है

Joel Tomkinson

Elizabeth Topp

Sarah Tsermengas

Matt Tucker

Kristie Uminn

Jason Unthank

Stephen Van Der Aa

Michael Van Treese

Stephanie VanTuyl

Julic Van Winkle

Jolene $V$ an Wingerden

Andrew Vargo

Scott Vaughar

Shauna Verosky

Naomi Vitt

Jessica Von Der Mehden

Gretchen Vuurens

Brittany Waggoner
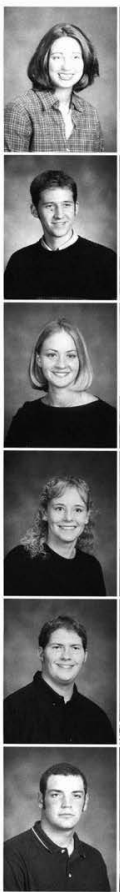

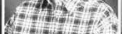

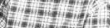
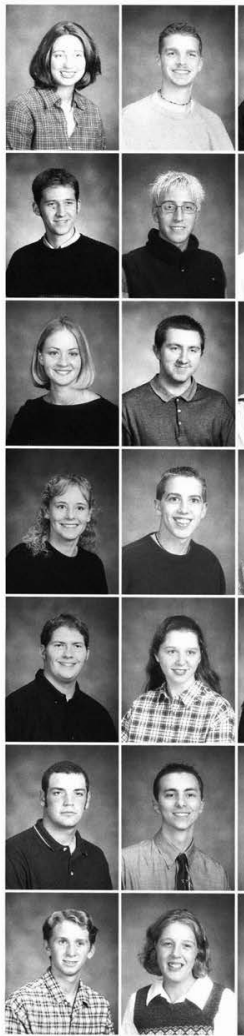
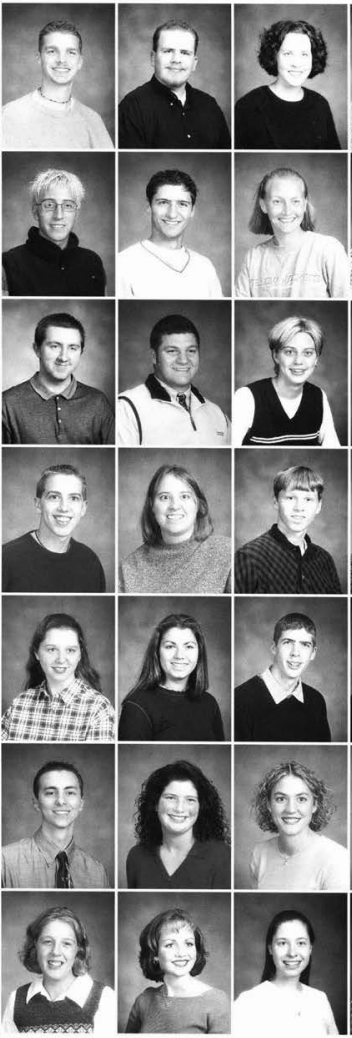
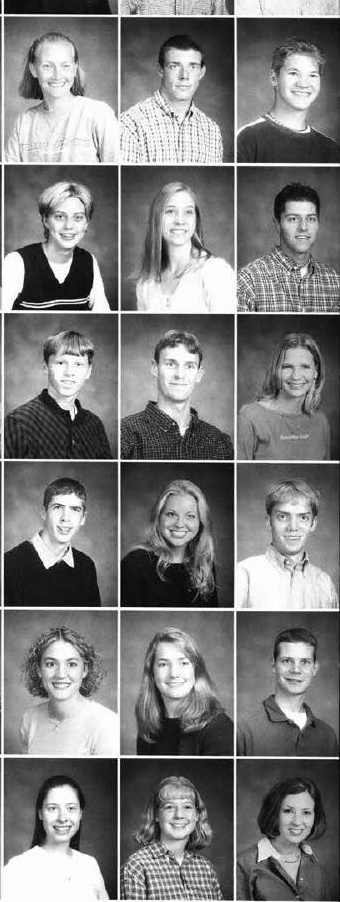


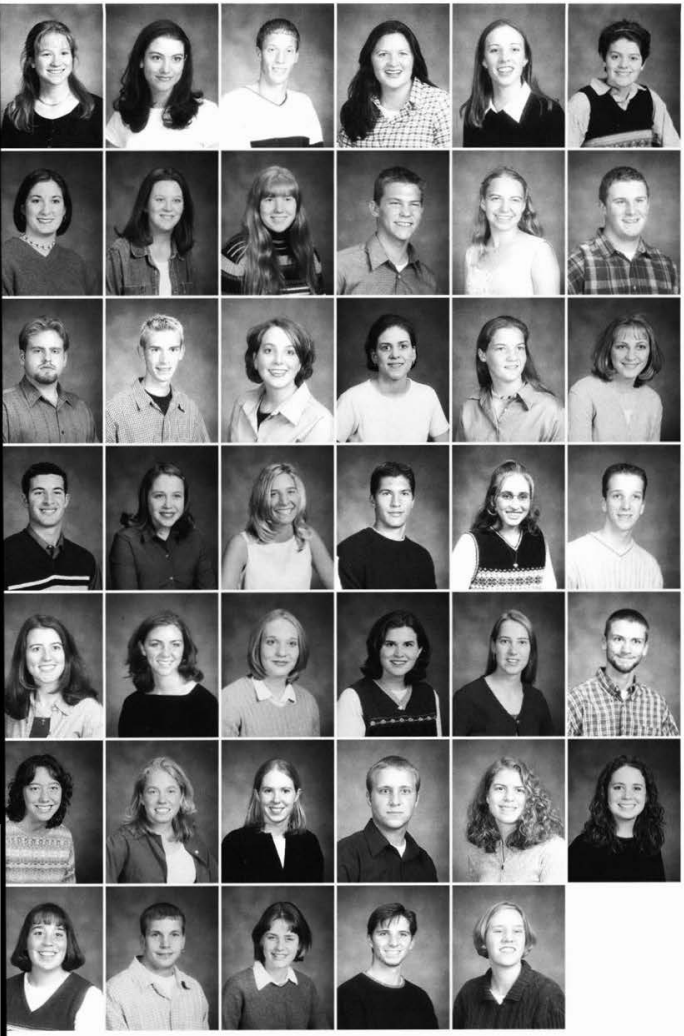

Karissa Waldron

Michelle Walker

Justin Walling

Sarah Walters

Esther Warts

Sarah Weaver

Erin Weber

Staci Weldon

Kelly Wentzell

David Wenzel

Heather Westbrook

Jason Wetsel

Zach Wheeler

Justin Whitaker

Megan Whitman

Mariette Widner

Amy Wiggershaus

Tricia Wilkens

Andrew Williams

Rachel F. Williams

Rachel J. Williams

Andrew Wilson

Jen Wilson

Corey Winger

Andrea Winship

Michal Witt

Sarah Woodstock

Heather Worth

Jennifer Wortley

David Wright

Gillian Wright

Heidi Wright

Sharon Wright

Rob Yale

Heidi Yehnert

Kristie Yerk

Heidi Yoder

Lance Yoder

Emily Young

Tim Zierenberg

Katherine Zimmerman 
Everyone who does evil hates the light, and will not come into the light for fear that fis deeds will be exposed... 


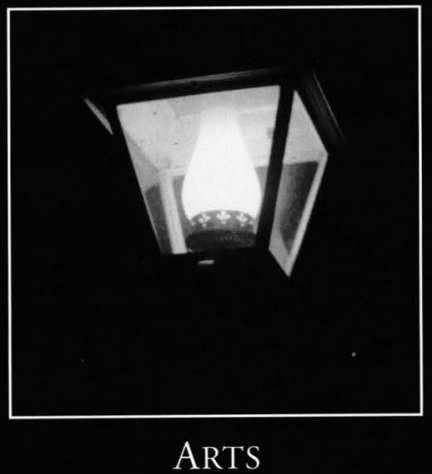




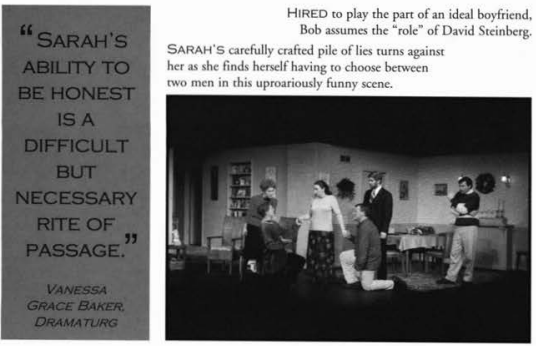

HIRED to play the part of an ideal boyfriend, Bob assumes the "role" of David Steinberg.
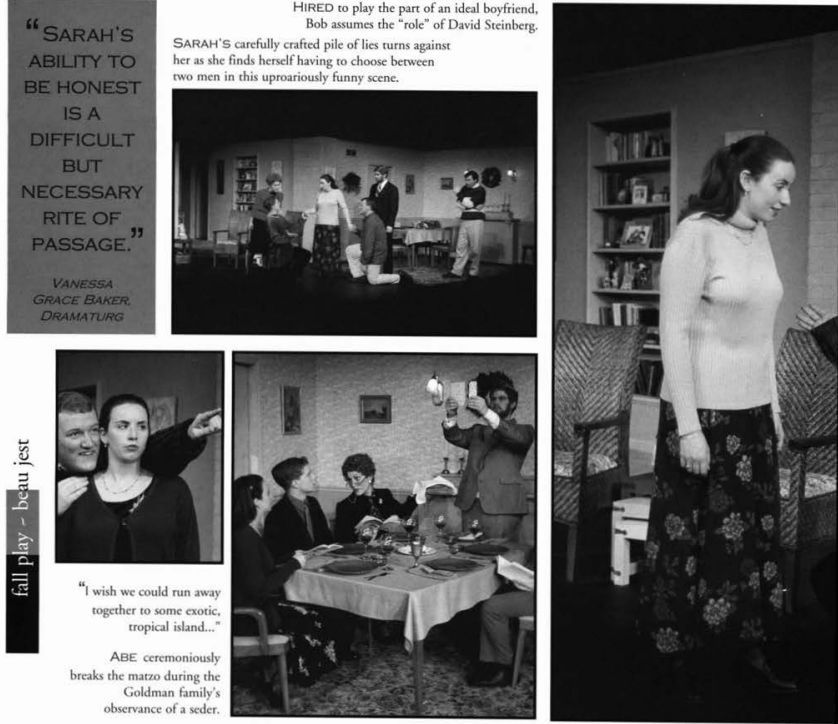

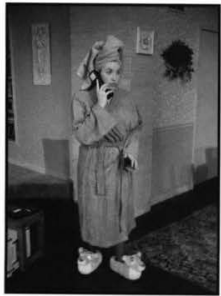

"...THINGS are very complicated right now..."
CLOCKWISE FROM TOP LEFT:

Greg Davis as CHRIS

Brenon Christofer as JOEL.

Jeremy Brown as $\mathrm{BOB}$

Christa Culler as SARAH GOIDMAN

Joanna Robinson as MIRIAM

Daniel Konopasakes ABE.

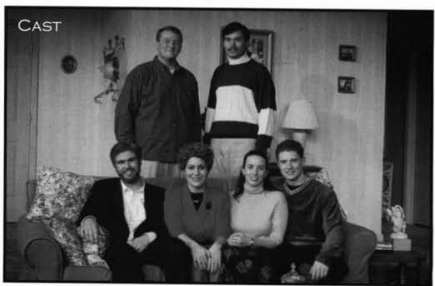

PRETENDING to be a Jewish doctor in order to please Sarah's parents, Bob finds himself in a rather uncomfortable situarion. 

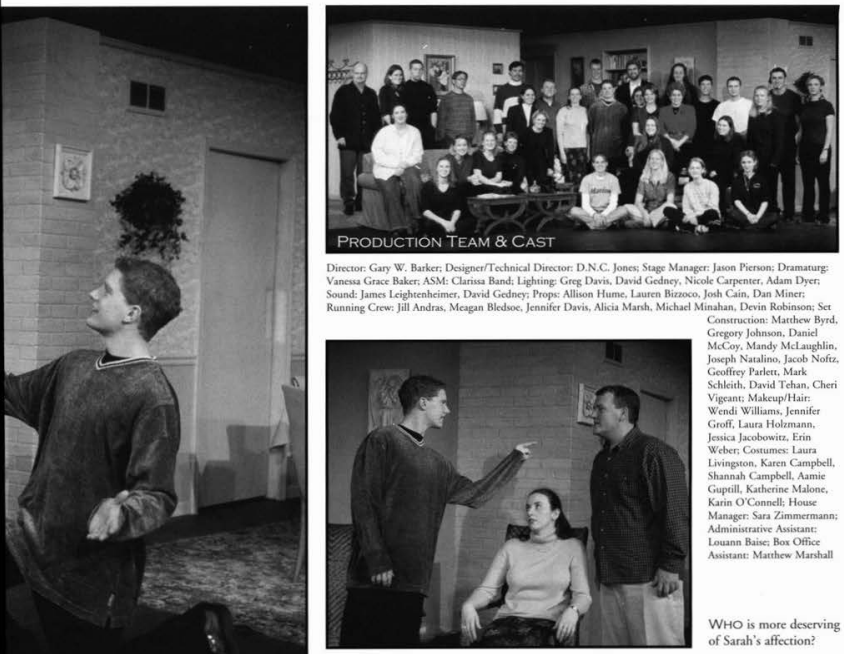

Director Gary W. Barker; Designer/Technical Director: D. N.C. Jones; Stage Manager: Jason Pierson: Dramarurg: Vanessa Grace Baker, ASM: Clarissa Band: Lighting: Greg Davis, David Gedncy, Nicole Carpenter, Adarn Dyer. Sound: James Leightenheimer, David Gedner, Props: Allison Hume, Lauren Birzoco, Josh Cain, Dan Miner; Runaing Crew: Jill Andras, Meagan Bledsoe, Jennifer Davis, Alicia Marsh. Michael Minahan, Devin Robinson; Set

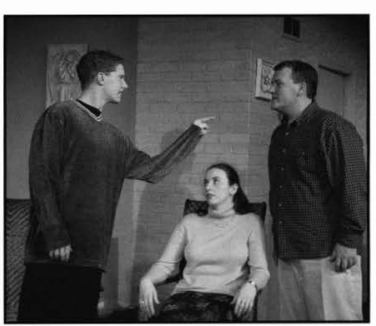
Constraction: Matthex Byrd, Gregory Johnson, Daniel McCoy. Mandy Mclaughlin. Joseph Naralino, Jacob Noftz, Geoffrey Parlett, Mark Schlcith. Darid Tehan, Cheri Vigeant; Makeup/Hair Wendi Williams, Jennifer Groff, Launa Holzmann, Jessica Jacobowirz, Erin Weber; Costumes; Laura Livingston. Karen Campbell Shannah Campbell. Aamic Guptill, Katherine Malone, Karin O'Connell; Horse Manager: Sara Zimmermann: Administrative Assistane: Louann Baise: Box Office. Assistant: Matuhew Marshall

WHO is more deserving of Sarah's affection?

\section{DRAMA DELIGHTS}

Autumn leaves and unseasonably warm weather set a pleasant atmosphere for this year's fall theatrical production of James Sherman's Beau Jest. As one of the highlights of Grandparents' weekend, this romantic comedy entertained multiple generations. Director Gary Barker said that "We chose Beau Jest because wewanted a light, family-friendly show that would be well-suited to Grandparents' weekend."

This light-hearted play tells the story of Sarah Goldman's desire to please her parents - even in her dating life. Sarah's Jewish parents, Abe and Miriam Goldman, want nothing more than their daughter'shappiness. They believe, however, that Sarah's happiness will only be complete when she dates a "good Jewish boy."

So as not to disappoint her parents, Sarah hires an actor, Robert Schroeder, whom she believes to be Jewish, to play the part of her new boyfriend. Humor escalates as Sarah learns that Bob is not a Jew and has virtually no background in Jewish traditions ot customs. Throughout a series of comical twists, turns, and developments, Sarah realizes the value of solid faith and honest relationships.

According to Barker, "This is a fun, romantic comedy that explores love, family, honesty, and belief. People will enjoy themselves." From the audiences' constant laughter, it appeared that Barker was, indeed, correct. 


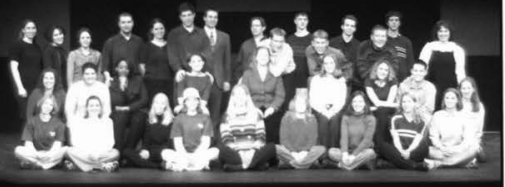

2

THE support erew working alongside the cast members and director, is absolutely essential to the success of any play production put on by the Cedarville Drama Department. The Crew specialized in tasks including, lighting, visual technology, costumes, musicians, set construction, properties, makeup/hair, sound, and administration.

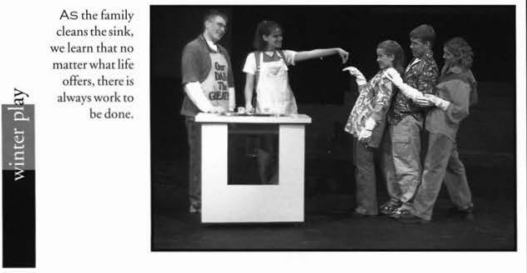

\section{DRAMA DEPARTMENT GIVES

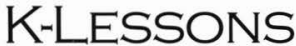

The winter play, Robert Fulghum's "All I Really Need to Know 1 Learned in Kindergarten," was a departure from the usual plot-centered play. It consisted of a series of vignettes which concentrated on "reflecting on life and love, growing up and growing old together." Actor April Lucas described her roles, saying, "I did not have a static, concrete role. Each scene was a different story, so at different times I was different things. My name was April throughout the entire play, but at one moment 1 would be five years old, and at another 1 would be in my late twentics or early thirties."

'Christmas/Valentine's Day' looked at an older married couple in which the husband was losing his memory, and 'Fathers and Sons' examined the tensions that exist in family relation. ships.

Actress Jennifer Wishart said, "1 think my favorite role was Donnie, the deaf boy who raked leaves for money. After he raked all the leaves, he threw a few up in the air as he walked home...just for the joy of seeing them fall. To me, that says that we can all find something to enjoy in life-no matter who we are or how many difficulties we have." Daniel Pierre, whose brother Jeremy was in the play, said, "I liked 'Problems and Inconveniences,' a scene in which a college student learns from a Holocaust survivor what the differ. ence between a problem and an inconvenience is."

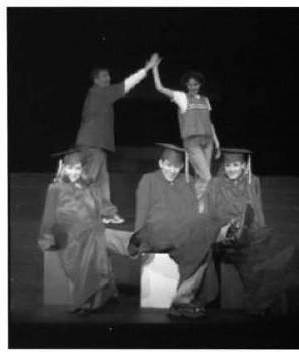

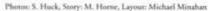


HAPPINESS is made of simple joys and not just toys was the theme of the vignette with three drivers from different walks of life.
CENTRAL in the sketch about Cinderella is the pig who made something out of nothing.
WHEN LIFE WAS

SIMPLE, WE

LEARNED

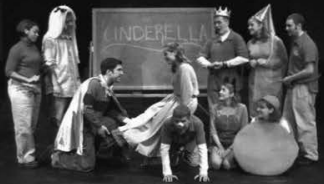

LESSONS

THAT STILL

CARRY

MEANING

FOR OUR

LIVES

TODAY.

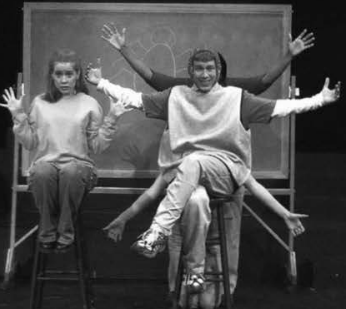

THE

lesson of the

spider and

the water

spout is one

that teaches

courage and

resiliancy.

\section{CAST}

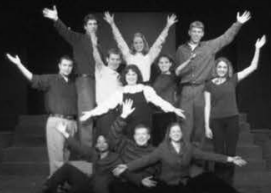

Row 1: April

Lucas, Ryan

Culpepper, Katie

Masterston

Row 2: Dan

Miner, David

Gedney, Dr. Baker.

Allison Hume, Julie

Van Winkle

Row 3: Icremy

Pierre, Jen Wistart.

Brain Coon

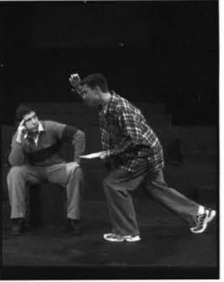

PROPER perspectives in life are essential to personal well being.

coupled with responsibility. 


"EACH
NIGHT, I
TRIED
SOMETHING
DIFFERENT
TO MAKE
THE SHOW
A LITTLE
MORE FUN
FOR EVERY
ONE.
JONATHANCULVER

TOUCHSTONE, the jester, falls in love with Audrey, the country girl, who

is incidentally decply loved by a common shepherd boy(right).

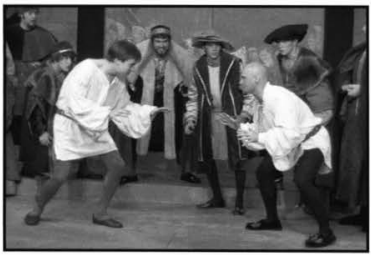

CHARLES, the court

wrestler, is hired to kill

Orlando, but loses the match

to the young lover(above).

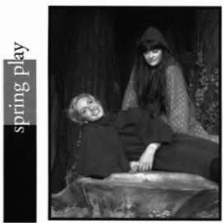

ROSALIND is accompanied by her best friend Celia after her banishment from her uncle's court.

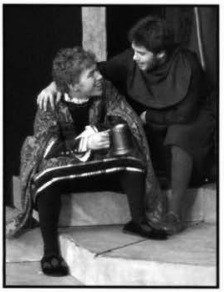

DisCuSSING the plans keeps these characters busy and cheer-

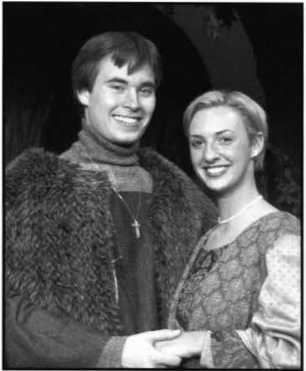

BRENNAN Christopher and Clarissa Band star as Orlando and Rosalind(above).

SYLVIUS demonstrates his undying love for Phoebe who has eyes for Ganymede.

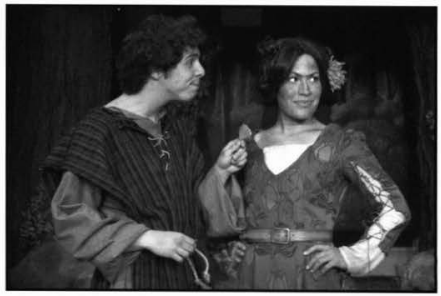

THE melancholy Jacques commends his sad words to those who would listen(right). 

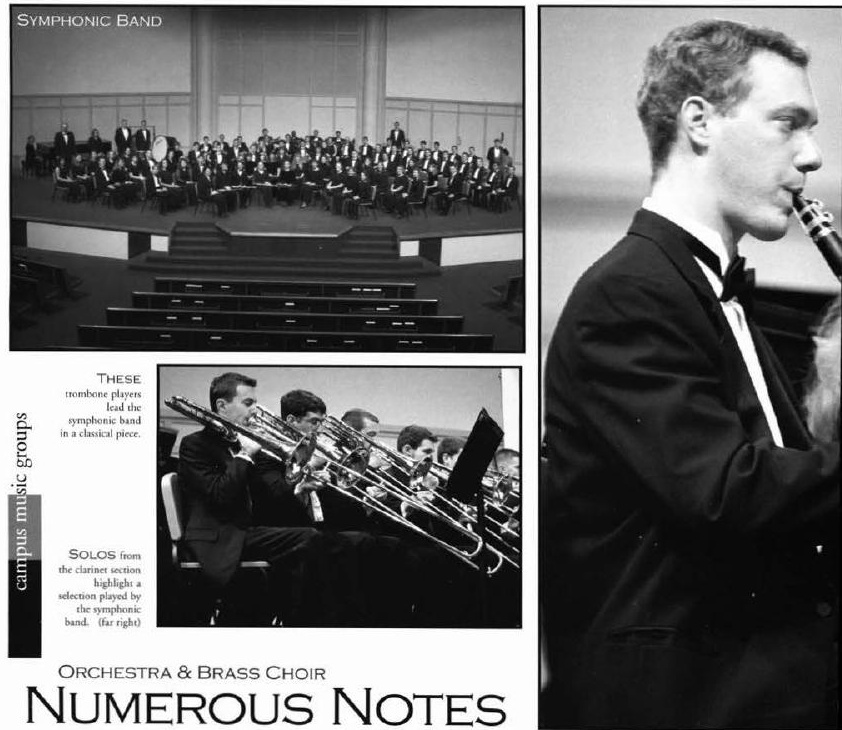

\section{ORCHESTRA \& BRASS CHOIR

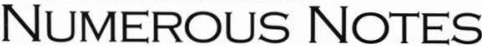

There were many things that contributed to the wide-ranging experience that Cedarville College provided its students. One of these contributions was in the area of music. Two specific instrumental groups in which students participated in were the orchestra and the brass choir. Under the direction of Professor Charles M. Pagnard. both groups entertained and enriched the students that participated by playing and also the students that participated by listening.

Representing a wide range of interests and pursuits, fifty-five Cedarville students composed the orchestra. In the past, the orchestra program had no consistent leadership and had to depend on various profes. sors for its direction. Eventually, the orchestra changed to involve only string instruments.

However, in the fall of 1997, when Professor Pagnard took over its directorship, he returned to a full-symphony orchestra. The pieces that the orchestra performed were primarily classical, but also include church orchestra ministry music and even pop music. The orchestra played at several events during the year including two concerts homecoming weekend, the Music Department Concert and the Twila Paris Concert. They also performed at the Fall Band/Orchestra Concert, the Showcase Concert, the annual spring Pop Concert, and various chapels.

Many of the members of the brass ch were in the orchestra as well. Pagnard co mented that "the brass choir was a very tigh knit group of CC students. Not only did th perform together, but they have also gr together as family."

The choir performed at almost all the sa concerts as the orchestra. In addition to campus performances, they also played local churches. The highlight for the ch was their performance in Gabrieli Wittenburg University on April 9.

Professor Pagnard felt that the brass ch made a significant contribution to the $n$ sic department in that it "provided so brass music on the campus of Cedarv College." 


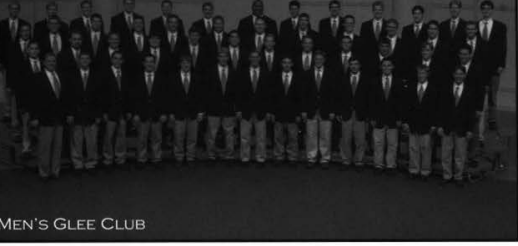

Men's Glee Club \& Jubilate

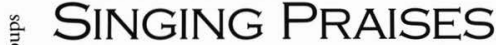

Jubilate and the Men's Glee Club ministered to the hearts and souls of all who had the privilege to listen to them sing. The groups sang a variety of music styles at various places and events. The students enjoyed a year of creating close friendships and growing together spiritually through the ministry of music. From concerts to tours, the students made memories that they will not soon forget.

Jubilate, led by Dr. Whaley, was a musical group of 44 vocalists who were selected by audition in the spring and fall. Jubilate helped students to lead worship in local churches and was not just for entertainment purposes. Justin Tubbs said, "Jubilate really caused me to look at

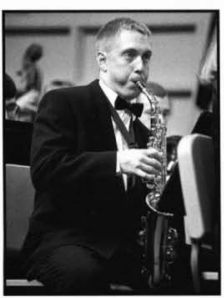

TiM Sutton can always be found entertaining audiences with his saxophone. worship in a new light. Dr. Whaley was very focused on worship and ensured that was the focal point of each concert." Jubilate took their annual tour to North and South Carolina during spring quarter.

The Men's Glee Club, led by Dr. Ander. son, had a membership of about 50 vocalists from a variety of majors. The vocalists audi. tioned for spots on the club in September and May. Glee Club performed on and off campus, from churches to sporting events Their annual tour consisted of three concerts at Ft. Thomas, Kentucky and Atlanta, Georgia where the group performed in three different churches. Aaron Roth said, "I really enjoyed being on Glee Club and getting to get to know the guys better."
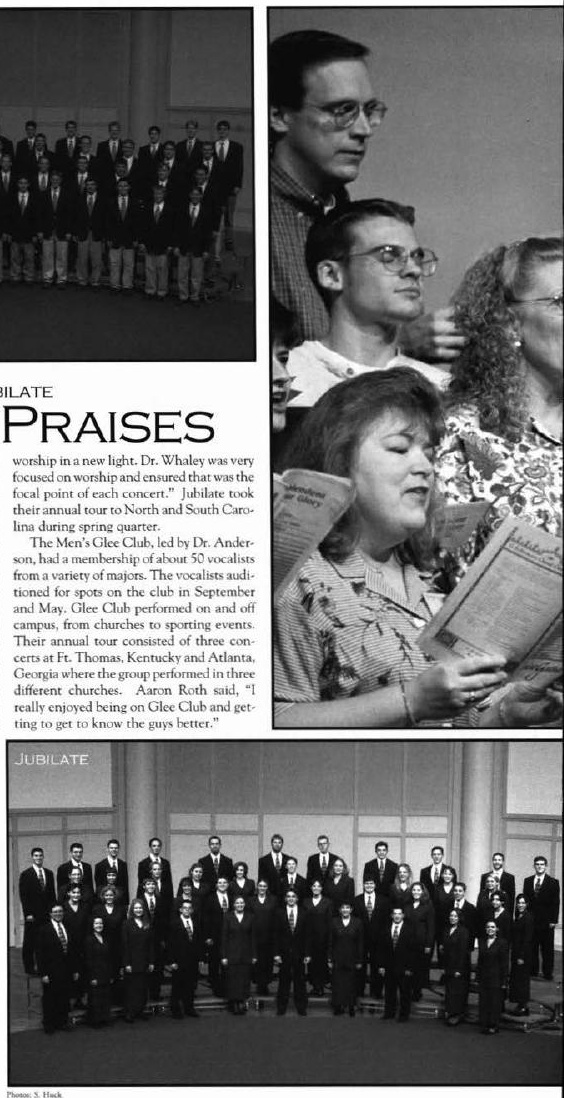

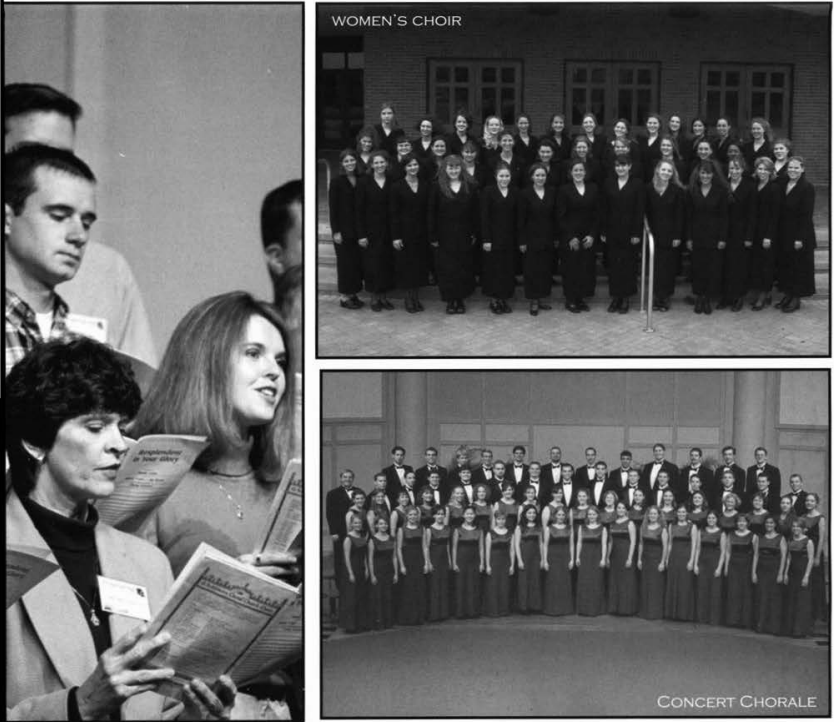

\section{ONCERT CHORALE \& WOMEN'S CHOIR}

\section{SINGING ON KEY}

Concert Chorale had another busy year, as they $\mathrm{g}$ at local schools, participated in a racial reconation conference with Wilberforce University, I traveled to Switzerland. "We've had some que opportunities," said Lyle Anderson, direcof the group since 1978. These opportunities luded giving assemblies at the high school in low Springsand singing with Wilberforce'schoir. e're white, and they're black," said Anderson. hd we're just Christians getting together."

Chorale members must learn a large number of sical selections. "Probably out of twenty-five ces of music, we'll only carry over a couple from previous year," Anderson explained. "The chalge of starting over at ground zero with a comtely new group was always fulfilling." The singseemed to agree with him. Senior Miriam Perez nmented, 'The variety of songs was fun. They got stuck in my head and I hummed them all day long."

Although Beth Cram Porter had only been directing the Women's Choir for three years, a lot had changedduring that time. The most noticeable change was its size-the choir grew from about 30 to its current number of 75 singers. Despite its size, choir members agreed that choir had an unusually high level of camaraderie. Senior Jill Strychalski said, "Women's Choir was more than just singing. It was being able to go in and talk with friends, laugh together, and share our hearts. We don't all know each other, but we all have a common bond through music." The choir widened its repertoire from sacred to pop, and also sang in churches, chapel services, the Pops concert and Showcase. "The choir's come so far with Mrs. Porter directing it," said senior lessica Wagner. "Her influence really made it a joy for us to be here."

Although Porter wanted her students to have fun, she also wanted them to work hard. "I learn and respond in a structured, fun and creatively sponcanecus environment," Porter said. "1 couldn't approach music in a stodgy, Puritanical, hands-inpockets way. I tried to get the choir to feel what I felt about music, which was very passionate. I certainly felt like I worked the singers hard, but that was the best-when you worked hard and still had fun."

Freshman Rachael Lewis commented, "We laughed a lot at Mrs. Porter's crazy antics,.,but we did a lot of work, too." Strychalski agreed. "She pushed us to do well, but she was also interested in our lives and wanted us to do well in life. I felt privileged to have been under her direction." 
... Gut whoever lives by the truth comes into the light, so that it may be seen plainly that what he fias done fias been done tfirough God. 


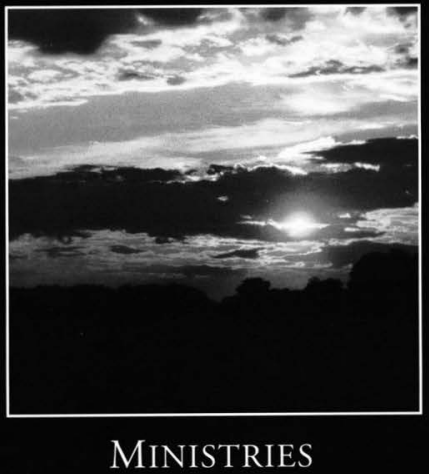




\section{ADVISORY SEVEY}

The Advisory Seven was a studentedected organization that worked to maintain the spiritual climate of the sludent body. The primary way they acheived this purpose was by erganizing and leading the fellowship services, held on Sunday and Wednesday evenings. This year the Ad 7 mernbers induded Michael Bodim, Tracy Commons, Josh Fronduti, James Melsger, Daniel Drice, Michad Shepherd, and Wes Tillett. These members were elected during winter quarter 1999 and served

"The Ad-7 gave us a unique place in which we could get to know students, build into each other's lives, and together grow closer to God." -Micbael Shepherd from spring quarter 1999 through winler quarler 2000 . James Melsger was appointed as the chairman of the Ad 7 by Daslop Rebenl Rehm, who also assisted the Ad $\tau$ with fellowship services and oversaw

their work. Josh Fronduli led mesic during the services, accompanied by several other singers and band members. During winler quarter 2000, Ad 7 organized and implemented a donation collection to raise money for seminary sludenls in Myanmar, Africa. This money was used to supply tedbooks to these sludenk, with the ultimale purpose of

expanding the culceach of the Gospel to the people of the world.

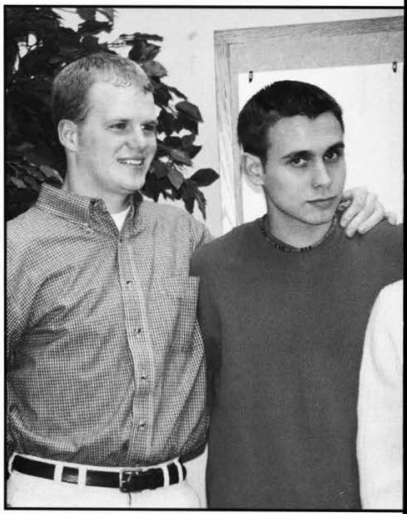

Dosler Rohm mentors Ad 7 throughour the year as they teach the student body.

Foed is a crucial part in planning meetings for $\mathrm{Ad} 7$. (below right)
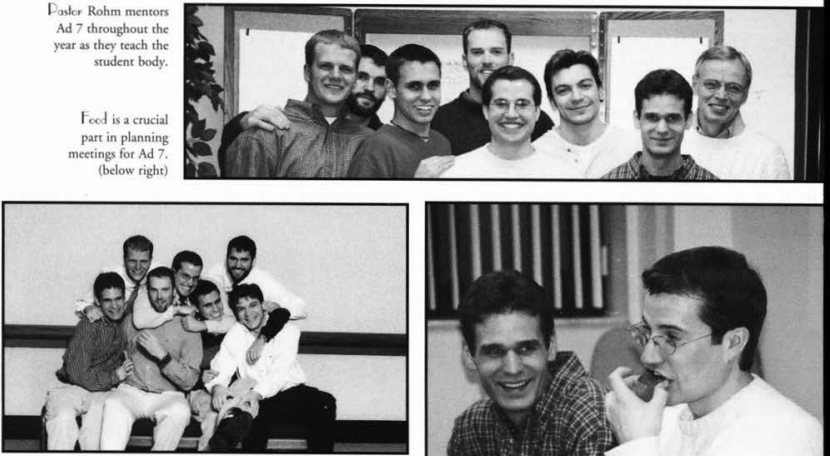

Row 1: Michael Shepherd, Tracy Commons, Daniel Price, Josh Fronduti, Row 2: Michael Boehm, James Metsger, Wes Tillett

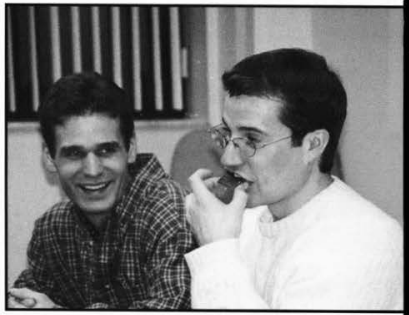




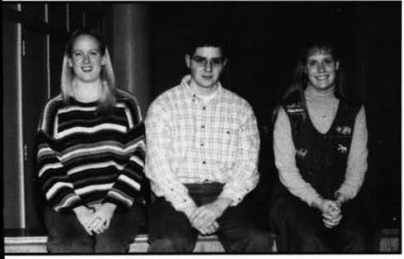

\section{AIRWAY BADTIST CHURCH} Children's Church

The Airway Baplist Church Children's Church team led children in church worship. They served on Sunday mornings by telling stcries, singing songs, and leading crafts with the chilliren.

Row 1: Nicole Carpenter, Chris Kelleher, Katherine Steingass

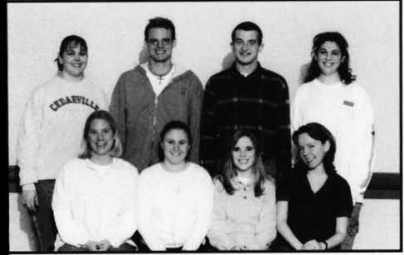

BEAVERCREEK BAPTIST $\mathrm{CHURCH}$

The Beavercreet Baplist Church leam enjoyed the opportunity minister to the kids through the Awana and nursery programs at

Beavercreet. Usually the bids ended up ministering to the leam more than they ministered to the hids. Team unily was an important part of this group's effectiveness.

Row 1: Becca Jensen, Emily Spangler, April Watson, Jamie Follweiler Row 2: Tammy Mikel, Justin

Hughes, Dan Hornbrook, Misty Smith

\section{BEREA BIBLE}

\section{Springfield}

The Berea Bible team halped the mambers of the church run the

Awana program.

The ministry involved working with kids ages 3 to 13 . It was a tlessing for the team members to ser how they could make a difference in the lives of dildren by taking a few hours a week to assist with the durach program.

Row 1: Patricia Hershberger Row 2: Christina Fullerton, Ken Yoder Row 3: Leanne Heath, John Scadding Row 4:

Andrew Jarvis,

Matt Goctz

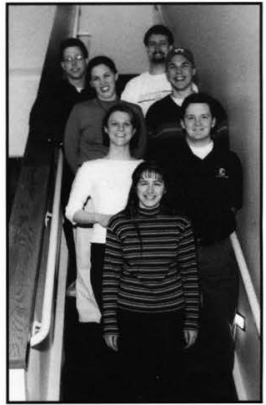

\section{Bethel BADTIST Springfield}

The Bethel Baplist leam was invclued with leaching the 4-year-olds through 6 th graders during Sunday School. They also assisted with the nursery, children's church, and junicr high group.

Row I: Lisa Smith, Greg Lason, Angela Rosscau Not pictured: Jami Broman

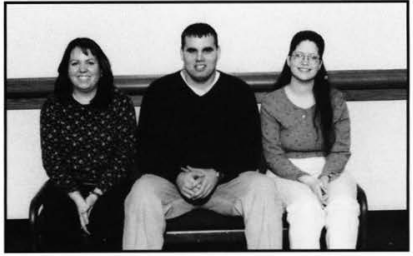




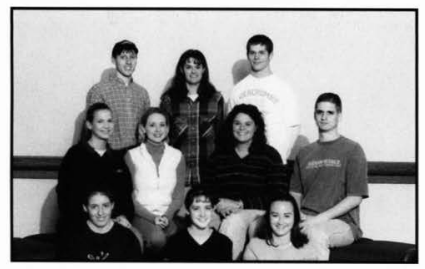

\section{BRISTOL HOUSE}

The Bristd I ouse Nursing Home was a visitalion ministry, in which the team members spent time personally getting to know the residents of the nursing home. The goal of their outreach was to share the Gospel with these individuals.

Row 1: Jean Osborne, Lisa Lauritzen, Heather Nicol Row 2: Erika Timm, Summer Allison, Kelly Kandel, Mike Brame Row 3: Andrew Frum, Christina Wheatley, Josh Johnson

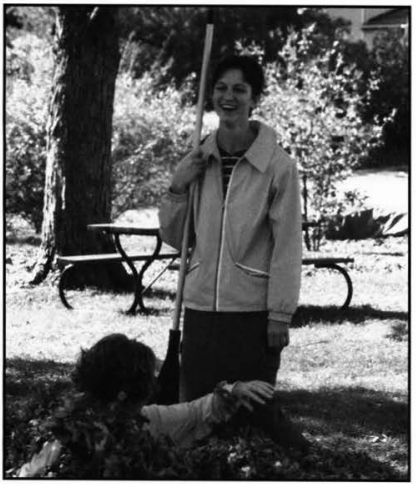

Raking leaves as a ministry team provides hours of exercise and fellowship, while serving community members with love.

\section{CEDARCLIFF EIDERLY ADARTMENTS}

The Cedardiff Elderly Apariment leam organized and led a Bible sludy and praise time with the residents. The minislry met weetly and was hosted by one of the residents who lived in the apariment complex.

Row 1: Marcella McCumber, Sarah Frohmberg, Tom Goodrow Row 2: Jeremy Maller, Scott Smith, Maribeth Mallman, Kristie Yerk
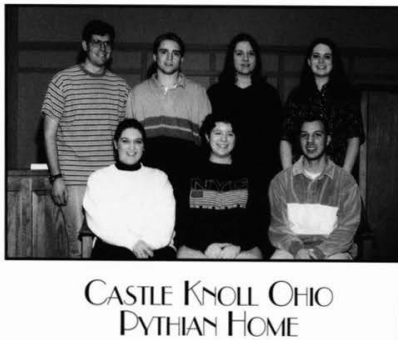

The Castle Knoll Team ministered to the residents of the Ohic Pythian Heme through worship services and visilation opportunities. In doing this, they strove to refled the love of their Savior and to encourage fellow Christians in their faith.

Row 1: Jennifer Apple, Tina Coon, Keith Gildow, Hannah Livingston, Erin Vallowe Row 2: Ariel Starbuck, Katie McGunnigal, Staci Weldon, Heidi Yehnert Row 3: Jeff Barrons Kate Schriemer, Elizabeth Uitti, Dan Nichols, Linda Dannemille

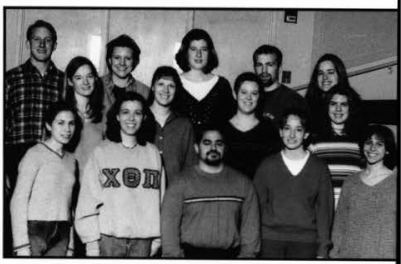




\section{CEDARKIDS}

The Cedarkids team involved ministering to lids in kindergarten rrough the sixth grade from the local communily. The ministry allempled to reach the kids through games, music, and Bible eaching, while developing a personal relationship with the kids.

Row 1: Kari Eaves, Kristi Beckett, Carrie Burke, Erika Bodden, Erin Smith, Erin Miller, Katie Koeppen, Danelle Ellis, Sarah Allen Row 2: Rebekah Manwiller, Todd Kocher, Ben Johnson, Charie Shifflett, Heather Albertson Not Pictured: Charles Corrick, Hedi Evert, Matt Carr

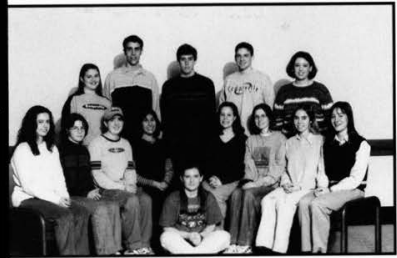

\section{CEDARVILE TUTORING} Elementary School

The Cedar Cliff tuloring team was dedicaled to assisting children with their school work while developing relationships. They focused on ministering to the children by showing them

Christ's lave through personal relationships.

Row 1: Jenney Flood, Amanda Oeffinger, Keri Herrold, Amber Durbin, Cameron Stephan Row 2: Greg Roth, Jena Mohr, Mark Boertje, David Drye Row 3: Philip. Preston, Sheila Fraser, Robyn Rausch, Josh Paulick

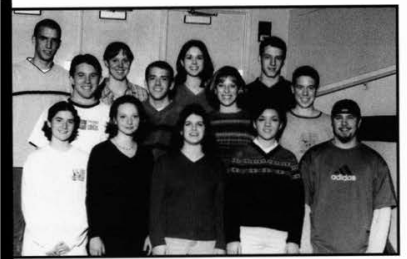

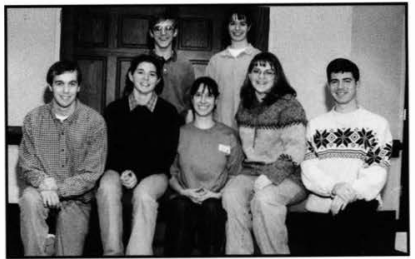

\section{CEDARVIIIF TUTORING High School}

The Cedarville Tutoring leam altempled to reach out to high school students through tutoring. They met with students at least Iwice a week. While there, they strove to become involved in not only the students' academic lives, but also their social lives.

Row 1: Donald Manchester, Sarah Polluck, Kimberly Eridon, Sarah Greene, Daniel Cross Row 2: Nathan Kibelbek, Laura Cochrane

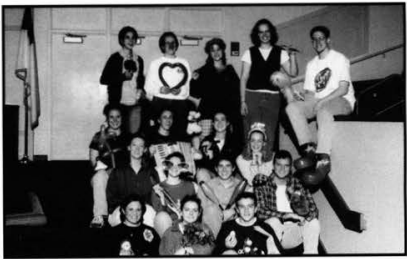

\section{CLOWNS FOR CHRIST}

The Clowns for Christ team ministered to children of all ages by providing a fun presentation of the Gospel. Typically, they performed a thirty to fourly minute program, which included exciling songs and skits and involved their clown characters.

Row 1: Kelly Gleason, Kim Harbison, Michael Burgman Row 2: Melissa Nuttall, Amber Tucker, Richard Brewer, Steve Olson, Bradley VanHeukelum Row 3: Ruth Wagner, Paul Thomas, Sarah Gayner, Amy Harris Row 4: Rebecca DeSantis, Celia Shortt, Melissa Cortina, Sandy Bennett 


\section{COLIFGE DARTNERS}

The College Dartners was a big brother/hiq sister ministry that met cne-cn-one with studenls from Cedardiff Elementary School. Typically, the leam members visited the children's hemes of look them cut in the community. The ministry was an opportunity to te a role-model and exmple of Christ's leve to hids in the community.

Row 1: Tiffany Grace, Julic Fraker, Jennifer Weaver, Jennifer Nelson Row 2: Meagan Bledsoe, Jenna Caron, Jessica Baralta, Ellen Ruby, Jim Llord Row 3: Stephanic Kreuder-Crowell, Amy Gregory, Danielle Rogers, Emily Young, Lissa Young Row 4: Lori

Carveth, John Mason, Kevin Carmichael, Ryan Foster

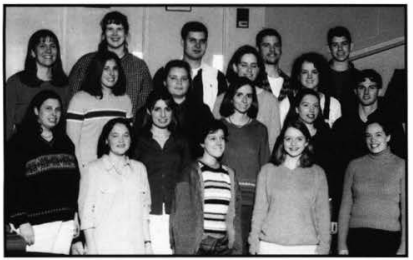

COLONIAL BAPTIST Awana

The Colonial Baplist Awana team sered with the purpose of building relalionships wilh young people and challenging

them in their pursuit of God. They worked with the

Awana praram and assisted in the youth group as mentors and brothers and sisters in Christ.

Row 1: Will Jenks, Shannon Grimm, Seth Finnegan

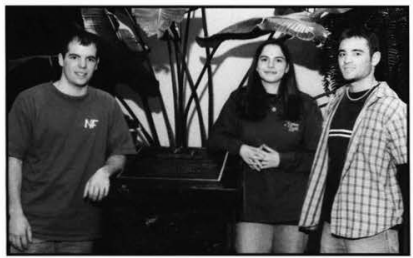

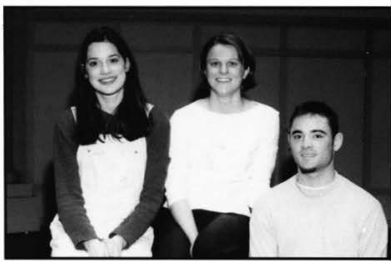

\section{COIONIAL BADTIST Children's Church}

The Colonial Baptist team served in the church by assisting with the Children's Church program. They provided childcare and ministered to the children of the church during Sunday morning worship services.

Row 1: Sharon Bullock, Christina Fullerton, Seth Finnegan Not Pictured: Brian Rosseau, David Tehan

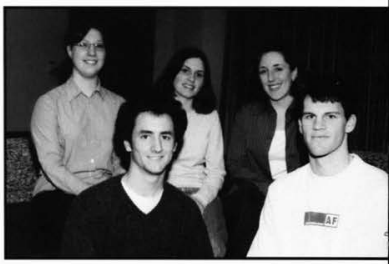

\section{CORNERSTONE BADIIST Springlield}

The Cornerstone Boplist leam laught dilldren's church on Sunday mornings and children's programs on Wednestay nigt Interaction with the 3-year-olds to fourth graders was a challen for the feam because of the wide aje span. The feam was amazed by the generosity of the hids throughoul the year, as th gathered pennies to support the missionaries of the durrch.

Row 1: Brittian Bollenbacher, Michael Keedy

Row 2: Laura Volpe, Karissa Walberg, Andrea Pattersan 


\section{COUNTY LINE BADTIST Dayton}

The Counly line Baplist team served in many capacities at the church. They worked with the children in Awana, Sunday school, dilldren's church, and youth group. They also ministered through special music presentalions. In addition, they ministered informally through fellowship with the church family.

Row 1: Kelly Barron, Sarah Wiersma, Rebekah Wiersma, Jessica Mathias, Kate Russell Row 2: Andy Smith, Joseph Mickey, Drew Meach, Tim Mohler, Jared Voigt, Joe Ciccanti

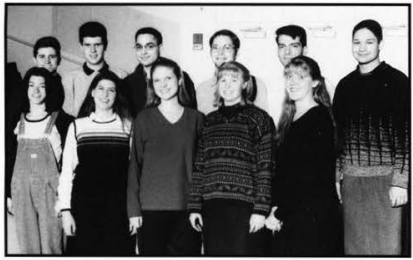

\section{DAVTON ChINESE ChRISTIAY CHURCH}

English Tuloring

The Daylon Chinese team taught English to Chinese people in Daylon each Sunday. Most of their students were unsaved qraduale or post-grad students. The team used the Gospel of John as their textbook.

Row 1: Sarah

Weaver, Emery

Nickerson, Linda

Knaues Row 2:

Allison Blosser,

Bethany Tate, Fsther Loh, Kristen Detwiler Row 3: Ben Clark. Rosemarie Marotta, Jan-Harm Wolters

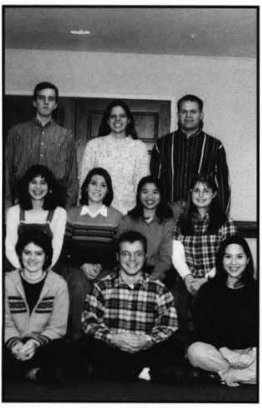

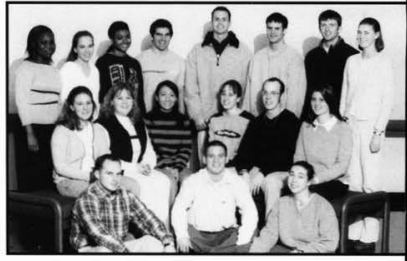

Dayton Detention Home

The Daylon Delention Home team traveled each Sunday to the home where they ministered to struggling teens through small grou interaclion. They led discussions, parlicipaled in a chapel service and led Bible sludies. The goal of this ministry was to bring the Gosped to a group that otherwise might nd have heard df Jesus Christ.

Row 1: Tim Sastic, Joshua Miller, Daniclle Parker Row 2:

Cheryl Gumprecht, Rachel Frey, Lina Ponder, Laura

Hummitzsch, Jacob Abbs, Nicolette King Row 3: Kikelomo Kolawole, Brittan Smith, Brionna Raynor, Jeremy Jeffries, Ben

Thomson, John Bourdeaux, Jason Covill, Sarah Grimme

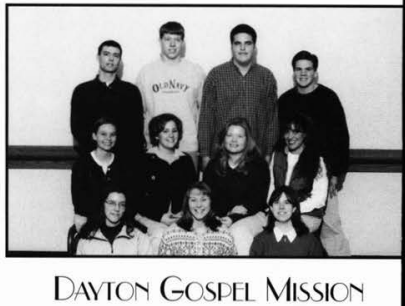

The Daylon Gospel Missien was an inner cily ministry team. They worked with the staff of the mission to serve meals and assist in the hitchen. They also worked with the children's ministry by helping with music, leaching, games, and adivities. In all things they sought to serve Christ and pul $\mathrm{I}$ im first.

Row 1: Meredith Keckrote, Kristin Schutter, Julie Trout Row 2 Abbey Beck, Julie Winar, Abby Dixon, Chanelle Deweese Row Adam Sticgelmeier, Chris Bartlet, Paul Abraham, Chris Lucarin Not Pictured: Joseph Natalino, Megan Bell, Ryan Roesler 


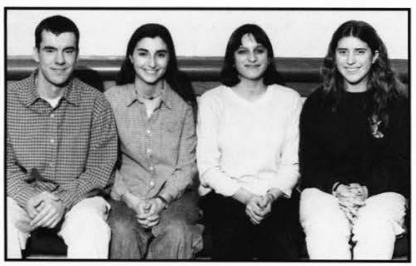

\section{FAITH BADTIST \\ Wilmington}

The Faith Baptist team taught Sunday school and assisted with the children's church program. They also provided special music for the worship services from time to time and assisled with nursery childeape as needed.

Row 1: Adam Bartlett, Jennifer Ingersoll, Jordon Natalino, Amanda Bustle

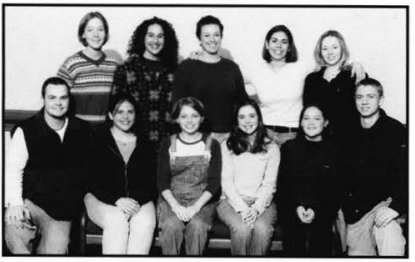

\section{FAR HILS BADIISI \\ Dayton}

The Far Hills Baplist team organized and led the Middle School Vouth group at the church. This imolved providing programs for the youth on Sunday mornings, Sunday nights, and Wednesday nights. In additien, the leam planned extra church adivilies for the youth qroup members.

Row 1: Phil Wing, Erin Gruber, Kimberly Schaffer, Renae Vogt, Tiffany Grace, Ben Rosner Row 2: Kim Wagner, Heidi Hambsch, Corban Garcia, Emily McQuinn, Amber Stenger

\section{FEIIOWSHID BADTIST Dublin}

The Fellowship Baplist leam taught Sunday school for children age two through fifth grade. They also assisled with the children's church, which provided services for children aqe Iwo through second grade. In addition, they helped with music by playing the piane and providing special music.

Row 1: Alison Camillo, Amy Twigg

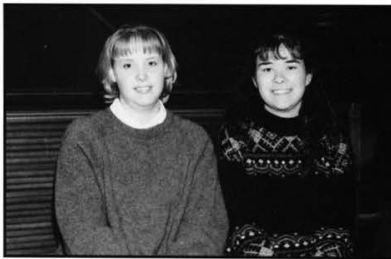

\section{FIRST BADTIST Enon}

The First Baplist leam provided support to the adult leaders of church's Awana

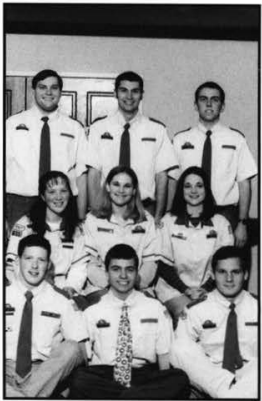
program. They helped out throughic the year by assistir with the game tim. counsel time, and book time. They a worked to become friends with the kid who came to Awar

Row 1: Benjamir Britton, Brian Hartsell, Chancy Persons Row 2: Sarah Britton, Meagan Smith, Nicole Smith Row Chris Grigson, Ala Brady Cotton, Da Dunham 


\section{FIRST BAPTIST Tipp City}

This ministry was in charge of the children's church lime.

They served on Sunday mornings by telling stories. singing songs, and leading crafls with the children.

Row 1: Susan Wallace, Amy Reed, Nikki Luckmann Row 2: Tom Lawson, Mark Underkofler, Jamie Hall

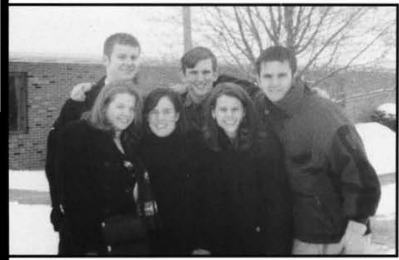

FIRST BADTIST

\section{Wilmington}

The First Baptist of Wilmington ministry team served at the hurch primarily by assisting with the Sunday morning worship. They layed instruments uch as the piane, organ, flute, and guilar during the congregational nging and provided special music. In addition, they ssisted from time lo time with the church's youth programs.

Row 1: Chris Recktenwald, Michelle Moore

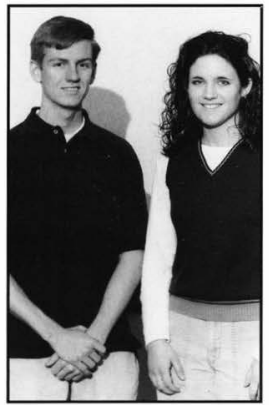

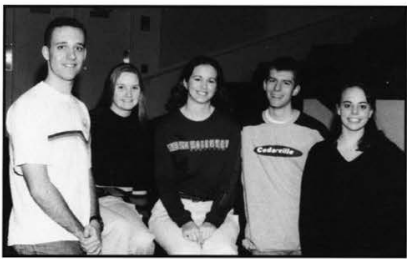

\section{FOREST RIDGE BADTIST Dayton}

The Forest Ridge Baplist team led the various clubs within the Awana program at the church. Their ministry was fwotold in nature because the leam ministered both to themselves by encouraging one another and also to the children and their parents by providing leadership for the Awana program.

Row 1: Timothy Kwast, Rachel Beth Kerr, Risha Stockton, David Bates, Michelle Ackerman

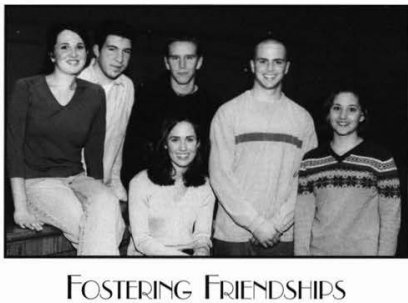

The Foslering Friendships team scught to help foster children feel loved in a unique way. They provided Christ-centered friendships culside of the children's foster families to encourage and minister to them. This ministry required full commilment, which offered these children stable relalionships from which they could draw strength.

Row 1: Sarah Leisure, Jeremy Pierre, Mary Deweese, Michael Coffey, Jason Olander, Kristen Hilsher 


\section{FrANKLIN StREET BADTIST}

The Franklin Street Baplist team was a multi-faceted team. Some of the team members were involved in Sunday school ministries, while others were imolved in children's church and special music. In addition, each of the leam members was involed in leading worship al one point throughoul the year.

Becky Stowers, Courtney Ludema, Christ Straits, Becky Thompson, Julie Henderson

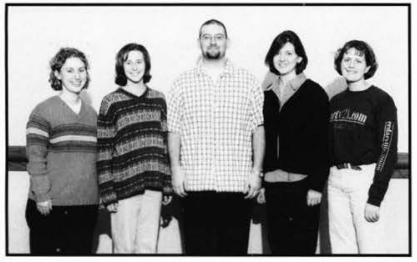

FRIENDSHIP BADTIST

The Friendship Baptist team traveled each Sunday night to the church, which was located near. Wilmington, to help with the church's Awana program. While there, they led songs, listened to children recile verses, and encouraged the church members in their ministry to the communily.

Row 1: Jen Wilson Row 2: Megan Mate, Jeremy Bessard, Christina Barrons

Row 3: John

Bolinger, Mike Wate, Jeff Oson

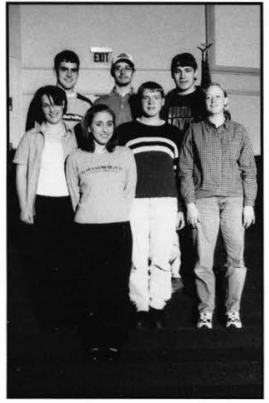

\section{GRACE BADTIST \\ Cedarville}

The Grace Baplist leam worled with the childnen of the church. They helped the hids mamorize verses, play games, and learn from special speakers. Each leam member loved kids and

fell privileged to serve them throughoul the year.

Row 1: Stephanie Van Tuyl, Kathryn Roberts, Angie Codey,

Hearher Tyson, Rachel Lewis Row 2: Jessica Snedeker,

Kelly Bernhard, Jessica von der Mehden, Andrew Williams,

Kenneth Waldock Row 3: Jan-Harm Wolters, Daniel Kwast, Kevin Haisch, James Trimble, Brett Cooley

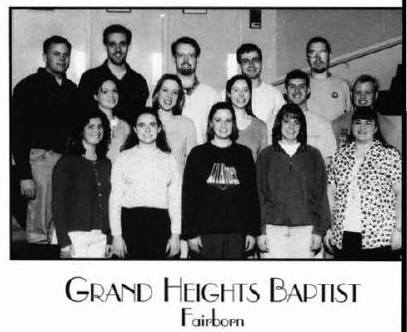

The Grand Heights Baptist leam worked in the nursery and two year-old to five-year-dd classes during the church service. The planned Bible lessons and sang songs with the kids. The leam members fell privileged to have been able to minister to the chure and become involved in the lives of the farmilies in the church.

Row 1: Sarah Rittgers, Joanna Carr, Katic Rosic, Sara Allen Row 2: Heather Stark, Aaron Mercer, Cliff Reynolds, Ben Stutzman, Susan Magin, Heather Roth

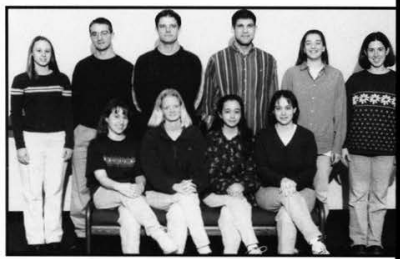




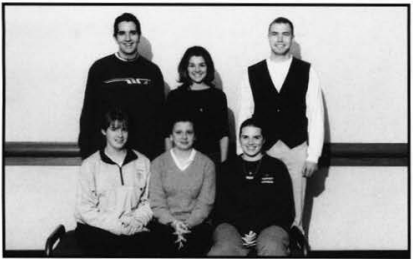

\section{GREeNe COUNTY COMMUNITY CeNter}

The Greene County Communily Center team served by visiling, tuloring, and reaching cut to the youth in the detention and probation programs. Their primary role was to listen to and counsel the leenagers in the detention center.

Row 1: Elizabeth Boer, Katie Powalski, Laura Gross Row 2: Paul Gwilt, Amy Wood, Micah Thirey

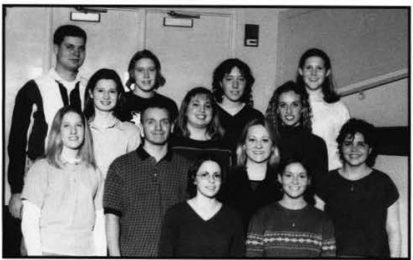

Grene COUNTY CRISIS DRFGMANCY CENTER

The Greene Counly CDC team worked to med the spirilual and physical needs of clients that came to the center. The leam members spent lime counseling dienls, leading the new abslinence program called STARS, and sorting and crganizing baby supplies.

Row 1: Cath Dato, Amber Corbin Row 2: Emily Elwell, Brendan McDonnell, Christine Compton, Rachelle Paquin Row 3: Teresa Ott, Jennifer Castellani, Lani Peterson Row 4́: John Mason, Kristen Killer, Gillian Wright, Whitney Johnson

\section{GREENE COUNTY RESIDENTIAL CENTER BOYS}

The Residential Center Boys team served as big trothers to the leenagers at the residential center. They were involved with activilies with the boys and also worked to build relationships with the tenns.

Row 1: Pete Springirth, Nathan Yorgey, Nate Gilbert, Lael Bryant Row 2: Ken Mansfield, Rob Marshall, Bob Kocher

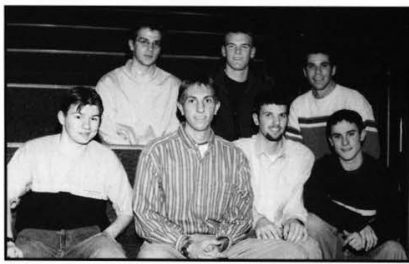

\section{GREENE COUNTY RESIDENTIAL CENTER GIRIS}

The Girsts Residential Center team was a big sister program that involved visiting the center each week.

The leam members planned aclivities and participaled in ene-on-one conversalional time. The leam strove to share Christ's love through their actions and words.

Row 1: Melissa Seward, Meg Copella, Rachel Beach, Amy Cliffe, Cara Snider

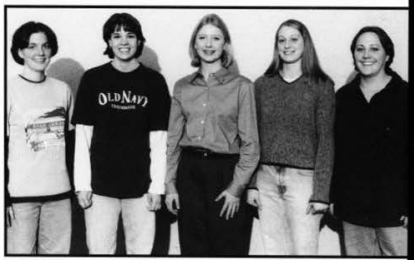




\section{HeATHERgRFene 1}

The $\mathrm{I}$ eather Greene 1 leam was primarily focused on encouraging the elderly spirilually. To do this, they sang songs, read verses, and prayed with the residents of the nursing home. The team fell that this time was encouraging for both the residents and the team members themselves.

Row 1: Bethany Bishop, Christopher Stokes, Mandy Herd

Row 2: David Hancock, Jamie Hall, Jeremy Shelford

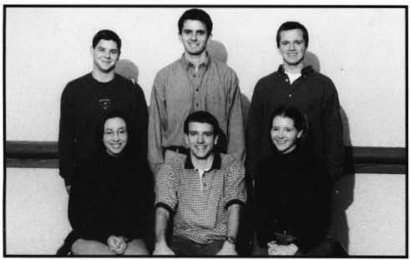

\section{HeATHERGREENE 2}

The Heathergreene 2 team was designed to reach out to the residents by visiling them individually. The leam members balled

with the residents about their spiritual lives and sang favorite hymns with the residents in the lobby.

The leam was impressed with the openness of the residents throughoul

the year.

Row 1: Joyce Bontrager,

Sharon Smith

Row 2:

Geneva

Roberts, Erin

Tuinstra,

Sonya Foster

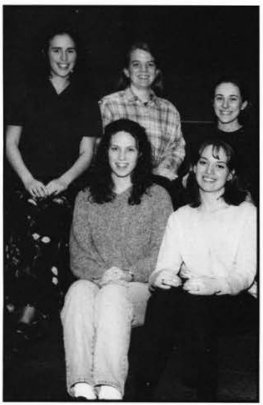

\section{Heritage FeIIOWSHID \\ Springfield}

The I eritage Fellowship team consisted of three parts: a worship leam which ministered

to the congregation

through music, a

kids' dub ministry. and a youth ministry.

The leam nd only had the chance to minister but also was thanktul for the opportunily to share with the church family.

Row 1: Christine Brittin, KristiLynn Kurezy Row 2: Paul Karlberg, Joshua Hynes, Mike Morgan

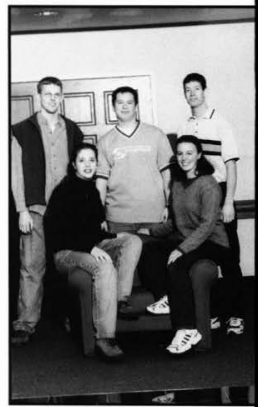

\section{HILLSIDE RETIREMENT HOME}

The Hillside Relirement team ministered to 15 residenls at an independent living home in $\mathrm{X}_{\text {enia. }}$ They organized Christmas ar Valenline's Day parties for the residenls. In addition, five of th residents attended a music department concert with the team in December. The leam learned that the residents loved to sing an listen to music and used this hnowledge to aid in their ministry.

Row 1: Kim Colyer, Nikki Sides, Jonathan Neu, Molly Rexford, Krista Baker

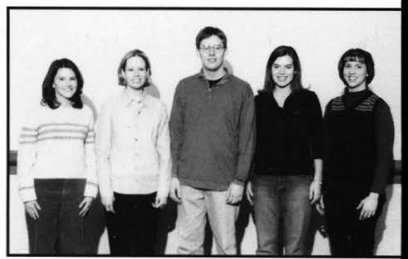




\section{Hode Evangeucal Free} Back 2 Back

The Bark 2 Bard leam was an outreach ministry in the Cinicinnali area that targeled eight high schools. The members worked "back to back" with the local church to bring high school students to the Lord and conned them with a local church. The leam led weelly discussion qroups, outreach activilies, discipleship meetings, and other relationship building events.

Row 1: Meg Jenista, Caleb Hague, Jessica Luz, Holly Favorite, Ryan Sparzak

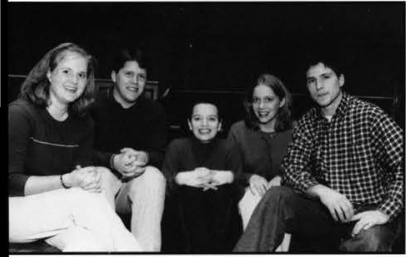

$\mathrm{IOOF}$

IOOF was a nursing home visilation ministry. The team members strove to encourage and minisler to the residents spiritually through music and evotions. They also worked to build ersonal relationships with the residents hrough one-en-one interaction.

Row 1: Kelly Reizz, Joyti Philip Row 2: Timmy Priano, Daren Norris

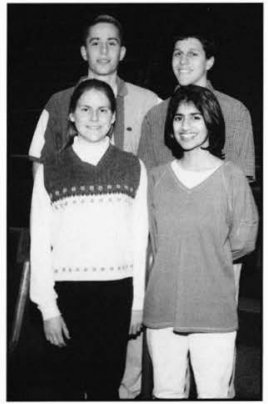

\section{MERCY HOSDITAL}

The Mercy Hospital leam had the opporlunity to serve a variety of individuals at the hopsital, including palients, stoff, and other volunteers. The ministry provided team members with an opperlunily to work and observe a hospilal setling, which was beneficial especially for those team members pursuing a carcer in the medical field.

Row 1: Lisa Hockenberry, Lisa Pendergrass, Jennifer Steely, Janene Pereira Row 2: Alice Rau, Mindi Sparks, Josh Logan, Jacob Dickinson, Karin O'Connell, Alicia Good

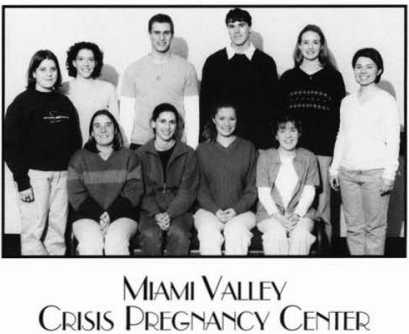

The Miami Valley CDC team assisted at the center in a variely of ways. They answered the carelines, helped to organize

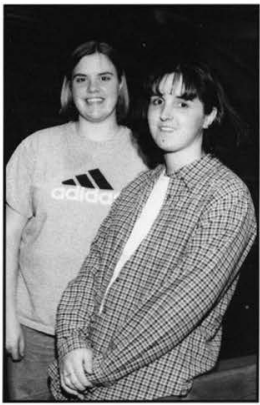
materials for dient services, and counseled women about abortion. If was exciling for the leam members to be able to gel to know many different women from many walks of life.

Row 1: Sarah Gilchrist, Heather Brown Not Pictured: Elizabeth Johnson,

Alicia Orlowski, Nicole Conn, Rachel Hamilton, Sarah Arnstein, Kristen Prince, Mel Mever 


\section{NORTHSIDE BADTIST \\ Awana}

The Northside Baplist team went each Wednesday nizht to the church to work with the bids who came to the Awana program. If was a blessing for the team to see the impact they were atle to hase on the lives of the bids. The leam strove to be Christ-lite examples for the hids.

Row 1: Megan Peterson, Erin Kuyper, Becky DeGarmo, Becky Schoonover, Nick Grisco, Julie Burt Row 2: Amy Morse, David

Perlow, Daniel Hacker, Shawn Conley, Emily Cronbaugh

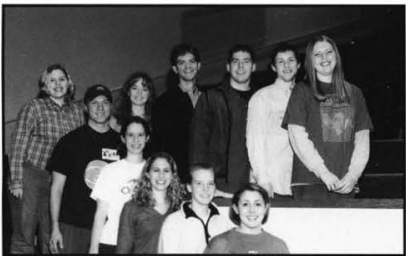

\section{NORTHSIDE BADTIST}

\section{Sunday School}

The Northside Baplist team faught children ages four through eight during Sunday School. By using shils, games, crafts, songs, and verses, they were able

to present the Gospel and instrud the hids on living a Christfilled life. Most of the hits came from broken homes which molivaled the leam to provide an example for them of people who try to lawe God with their liws.

Row 1: Patience Burkholder,

Maranatha Rubet,

Tabitha Rowe

Row 2: Mark Kirby, Daniel Konopasek.

Rett Strasler, Katic Jackson

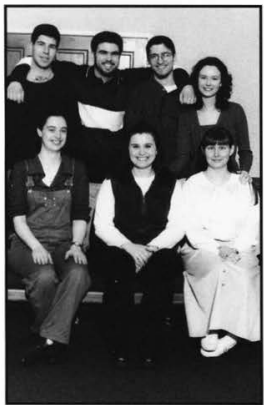

\section{NORTHWEST BIBIE CHURCH Children's Church}

The Northside Bitle team ministered to the children of the church during the Sunday

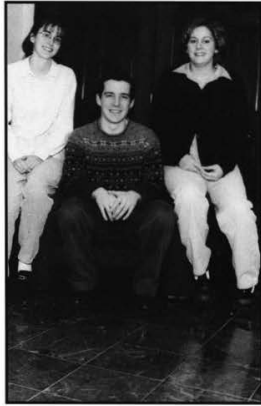
morning worship service. They used songs, games, lessons, and crafts to impad the children with the Gospel. B cultivating a loving environment, the team worked to communicate the excilement and joy that comes from living a Christ-filled life.

Row 1: Ellen Laber, Zach Rhodes, Julic Winar

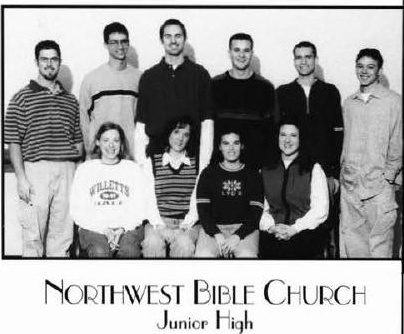

The Northwest Bible leam served the church by providing an effective junior high youth program. The team of ten sludents provided music, qames, and spealers for the students. Most imporantly, the leam built relationships with the students and strove to provide solid role models for them by displaying an inlense love for Jesus Christ.

Row 1: Rachel Bontrager, Jamie Rirzer, Leah Hendricks, Andrea Endicott Row 2: Steve Light, Matt Hofmann, Grady Peeler, Mike Call, Jordan Patrick, Jason Siemer 
The Northwest Bible Church Junior High team assists with a chain tube ride down the snow-covered hills at Scioto Hills.

tudents at the Barn enjoy the opportunity spend their Friday evenings talking, laying games, and hanging out.
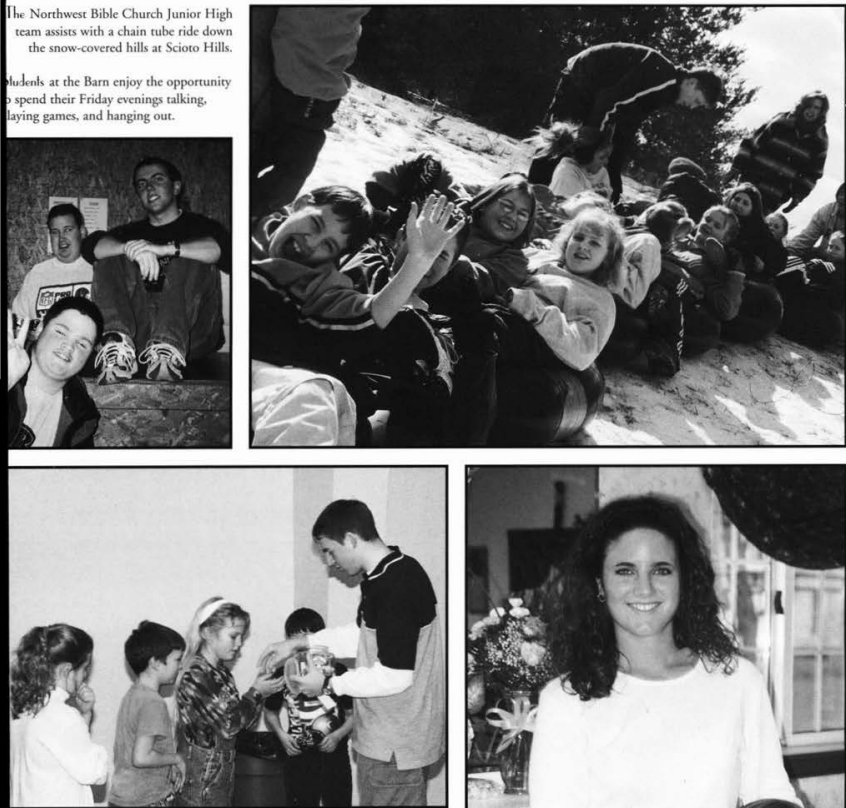

Cedarkids team members enjoy rewarding their students who are doing a good job.

Abigal Beck voluntecrs at the Dayton Gospel Mission, which allows her to work with young children, such as these two shown with her.
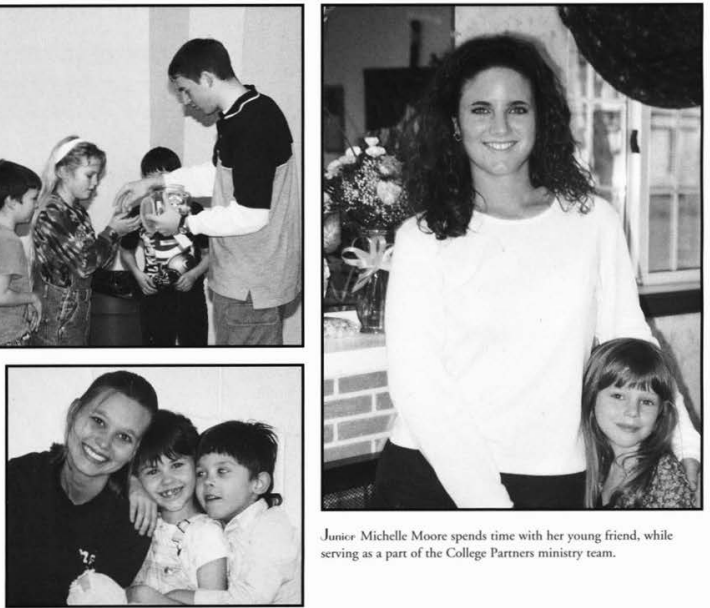

Janior Michelle Moore spends time with her young friend, while scrving as a part of the College Partners ministry team. 

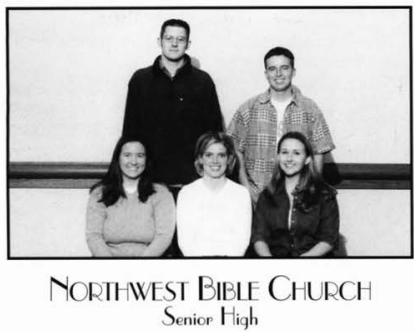

The Yorthwest Bible team assisted with the senior high youth programs at the church. They organized and led game aclivilies for the meelings to help the students fed more comfortable with one another. They also performed dramas for the youth group, which strove to reveal God's truth to the students.

Row 1: Jill Cross, Andrea Linamen, Julie Dugan Row 2: Matthew Peeler, Ben Gayer

\section{ODERATION REBIRTH}

The Operation Rebirlh team weetly visited a group d troutled adolescent boys. They strove to develop friendships and be godly examples for the boys through lessons, qames, and one-on-one inleraction.

Row 1: Heather Kemp, Rachel Burke, Jennifer Ament Row 2: Brianna Auport, David Dingeman, Josh Rowland, Megan Bcll

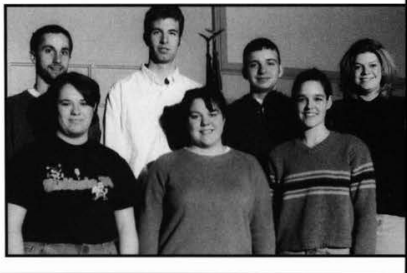

\section{ODFY HeIRS}

\section{Open I eirs was a beld stred} evangelism ministry to people on the streets of Cincinnali, Daylen, and

Columbus. The leam strove lo dey Christ's command to go into the entire world preaching the gospel and making disciples. The ministry provided its members an opporlunily

to live out their faith practically.

Row 1: Megan Hause, Lori Turner, Megan Borton, Sheli Oberbeck, Molly McClure, Karen Leininger, Michelle Horne, Jenny Maass, Jean Osborne, Lena Fischer, Rachel Regner, Julic Landau, Erin Ewig, DIXON MINISTRY CENTER

Lindsay Kaiser, Kristen Davis Row 2: Tara Cryan, Carissa Smith, Katie Zimmerman, Megan Whitman, Trent Hamilton, Beth Coolet, Larua Hummitzsch, Erin Collowe, Jodi Crawford, Charlotte Hager, Amanda Beattie, Dana Litchfield, Matthew Smith, Caleb Halulko, Eric Mitchell, Aaron Dieringer, Ben Phillips, Sawnna Moberg Row 3: Srephanic Carton, Rubie Mayburt, Katic Livingston, Will Jenks, L Bacerra, Adria Curts, Emily Gayer, Karen Keika, Angela Rosseau, Trisha Wabeke, Diana Locke, Kate Schriemer, Lisa Pendergrass, Nathan Boone, Shannon Lauer, Jesse Magnuson, Joel Hollins Row 4: Ben Mitchell, Hannah Livingston, Hanna Cook, Veronica Lawton, Debbie Perez, Eric Wentz, Daniel Olson, Aleesha Hager, Matthew McCracken, Jena Burns, Natalie Findley, Ruth Wagner. Tim Cochrell, Randall Bowens, Matthew Nelson, Christy Herma Row 5: Heath Huskey, Rachel Williams, Matt Hofmann, Adam Craig, Kyle Prowirz, Dana Gerber, Jared Applegate, Kyle Gerber Dan Sudlow, Joc Hime, Brock Weston, Charles Corrick, Willy Shelton, Shanna Moberg, Joel Estes 


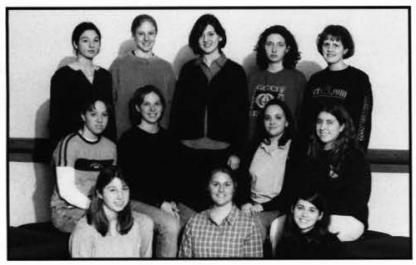

\section{SDRINGFIEID Crisis DREgnanCy Center}

The Springfield CDC team was able to speak individually to girls in crisis pregnancy situations. Many of the team members trained throughout the year to be volunieer counselors for the cenler. The leam enjoyed the opporunily they had to share with the girls at the center the hope that they can have in Christ.

Row 1: Diana Locke, Katic Kepner, Jennifer Wood Row 2: Alyssa Rost, Mary Evans, Christine Trages, Amanda Bustle Row 3: Cheri Viveant, Sarah Posegate, Becky Lynn Thompson, Davina Murray, Julie Henderson Not Pictured: Carherine Wayne

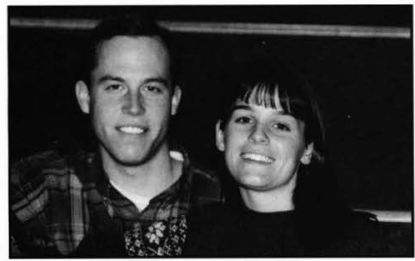

\section{ST. JOHN'S CENTER}

The St. John's Center team worked with elderly and disabled people at the center. While there, the team assisted individuals by leaching them how to increase their computer stills. The feam also strove to encourage the individuals al the center through personal interaction.

\section{THE ARK}

The Ark leam was a new ministry this year. They served by reaching out to inner caly children in Springfield. At the Ask, team helped to serve the children dinner. They also assisted by organizing and leading the children in games and Bible lessons

Row 1: Jessica Mao, Jayme Brower, Nick Miller, Tom Cizauskas, Betsy Linnell

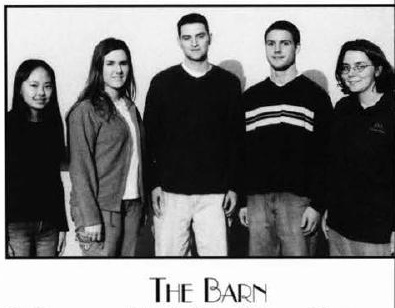

The Barn team worked at the Barn, which was a Christian you center welcoming a wide variely of sludents. The purpose of thit ministry was to develop relalionships with the teens at the Barn The team did this by playing qames, talking, and interacting wi the students. Their main goal was to love the teens at the cente and begin to share with them the message of the Gospel.

Row 1: Beth Brown, Karen Robinson, Kristi Schutter Row 2: Debbic Huff, Sarah Baker, Amanda Oeffinger, Allison Edward: Megan Hueni, Rejeeana Wright, Rebekah Wiersma, Jessica Math Row 3: Amanda Flenar, Glori Goheen, Bethany Keilman, Davi Drye, Ryan Stern, Josh Paulick, Dan Dunham, Bob Kocher, Greg R

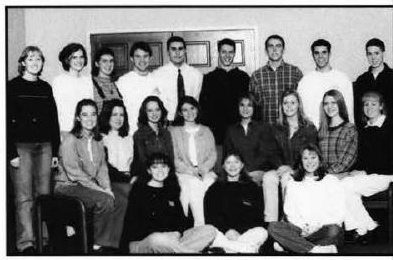




\section{YOUNG LIFE}

The Voung Life team sened as part of a high school outreach ministry that was based on building relalionships with bids. The purpose of these relalionships was to shew the love of Christ. The leam used songs, humer, shils, and messazes to reach, "the farthest cul kid."

Row 1: Darcy Fivek, Jenny Bonham, Emily Gray, Corrine Andrews, Melissa Foote, Erin Flora, Stephanie Strong, Kelly McKay Row 2: Jonathan Grisson, Jerred Davis

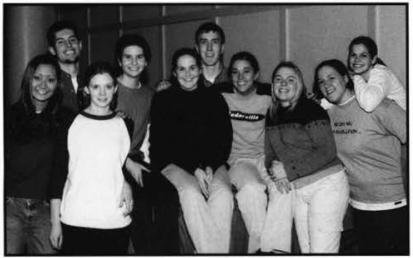

\section{ZION'S LIGHT TUTORING}

The Zion's I ighl team visiled Russian Jewish immigrants each week. They strove to build friendships through luloring the immigronts in English. While building friendships, the leam alse shared the truth of the gospel.

Row 1: Emma Chmura, Patty Roach, Becca Nasman, Kristi-Lynn Kurczy Row 2: Joanna Bupp, Rachel Menz, Erin McDaniel, Carolyn Sprankle, Brandi Elias, Robyn Cannon Row 3: Sara Gedraitis, Rachel Morris, Jon Shrubsele, Ben Lyons, Aaron Mercer

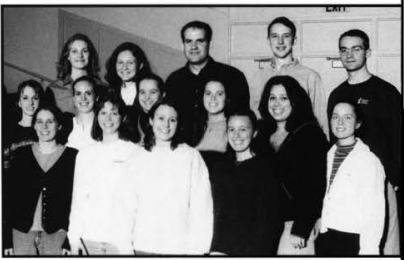

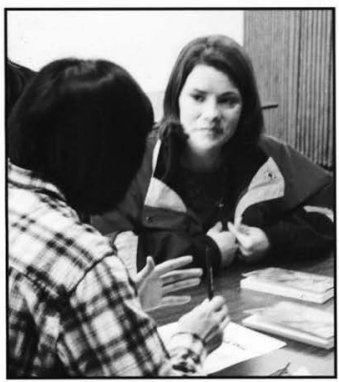

Bdhany Tate listens to a member of the Dayton Chinese Church, where the tutoring team uses the Gospel to teach English.
Menters of the World Missions Fellowship volunteer their time to perform service projects, such as raking lawns.

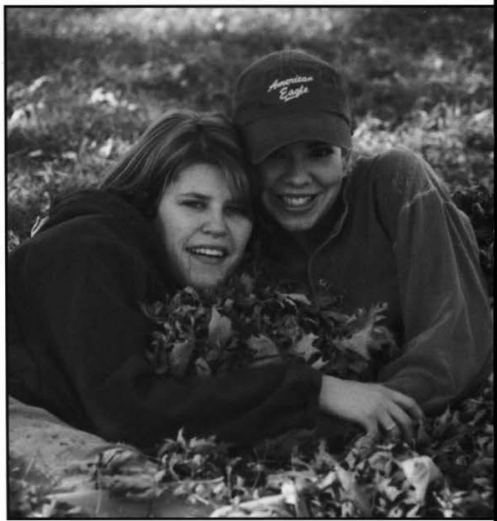




\section{TOURING TEAMS}

\section{ABUNDANT LIFE}

The Abundant Life leam was a singing leam that consisted of seven singers, two leyboard players, a quitar player, and a sound technician. They traveled throughout the school year to various

churches in the surrounding area. In the summer, they loured the southwestern stales, singing and serving local churches.

Row 1: Danielle Hatfield, Nikki Fortener, Jason Smith, Kristina Bilodeau Row 2: Jonarhan Kleis, Kristin Neumann, Kyle Estepp, Kara Davis Row 3: Doug Rotman, Jana Glessner, Nick Powell
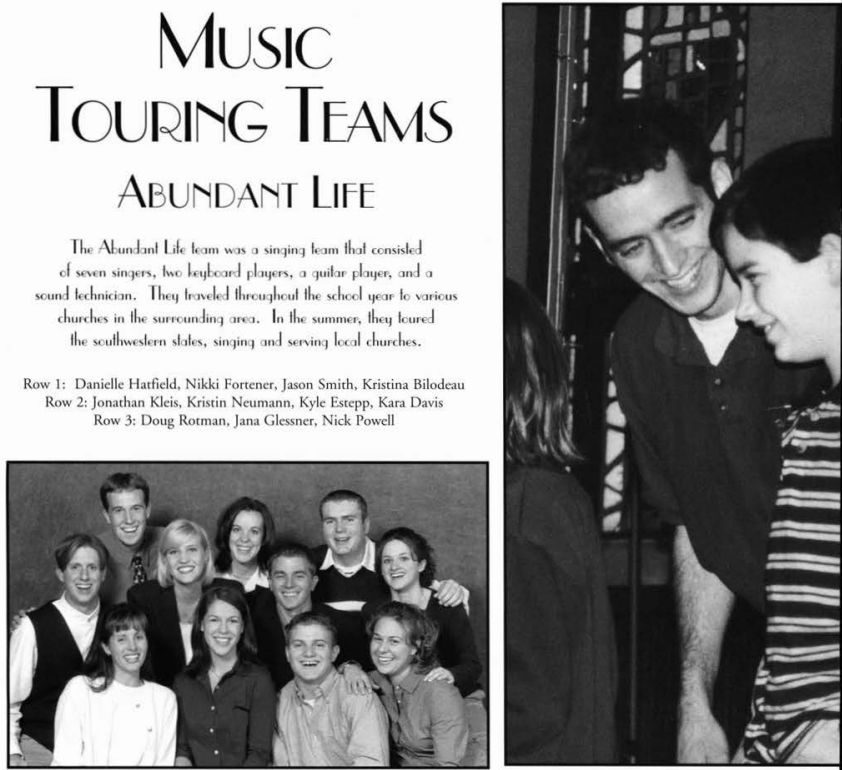

\section{KINGSMEN QUARIEI}

The Kingsmen Quardet was a traveling team with four singers, a keyboard player, and a sound technician. They traveled weetends throughout the school year, during spring break, and alse on an eight weet summer lour to the scutheastern states. The quartet used a variely of music that included southern gospel favorites in addition to lestimonies and Scripture.

Row 1: Justin Dyer, Andrew Rodriguez, Nick Tynan Row 2: Ben Whipple, Scott Moodie, Adam Dennis

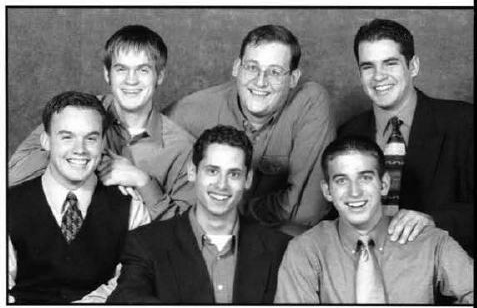




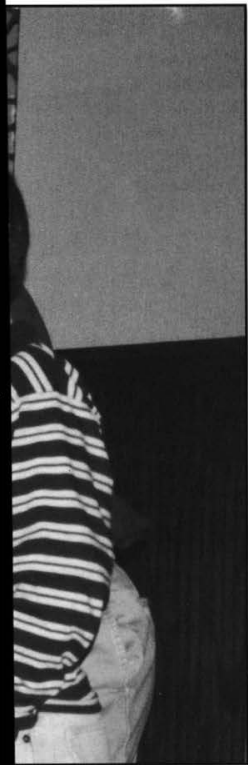

Mark Collins shows children how much fun

being on the stage can be.
Swordtearer, Lindsay

Wagner, sings a solo to a

church while on tour.

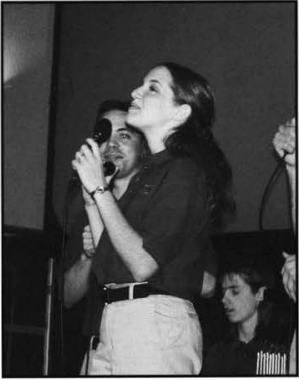

Kule Estepp, member of

Abundant Life, takes time in his

day to minister to children.
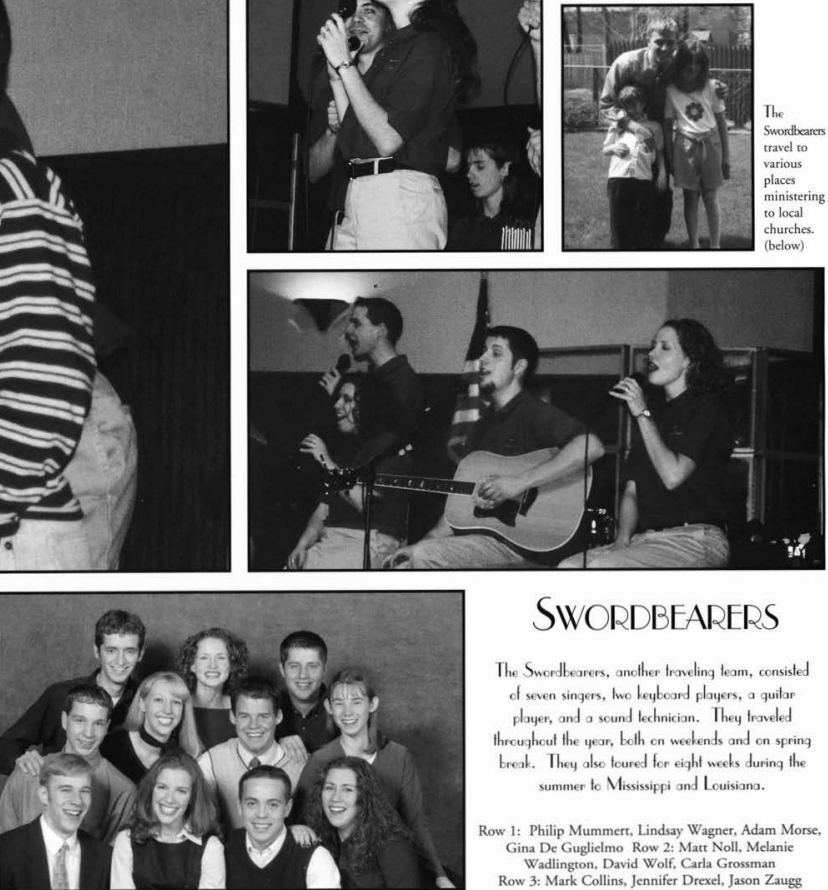

\section{SWORDBEARERS}

The Swordtearers, another traveling team, consisted of seven singers, two keyboard players, a quilar player, and a sound technician. They traveled throughoul the year, both on weekends and on spring break. They also loured for eight weeks during the summer to Mississippi and Louisiana.

Row 1: Philip Mummert, Lindsay Wagner, Adam Morse, Gina De Guglielmo Row 2: Matt Noll, Melanie

Wadlington, David Wolf, Carla Grossman Row 3: Mark Collins, Jennifer Drexel, Jason Zaugg 
Mermbers of Masters

Puppets 2 are bonded

together as a team.
Casey, Platt ministers to children of a church through the use of puppets.

Lifeline Players travel to use drama as a tool while ministering to audiences of all ages.
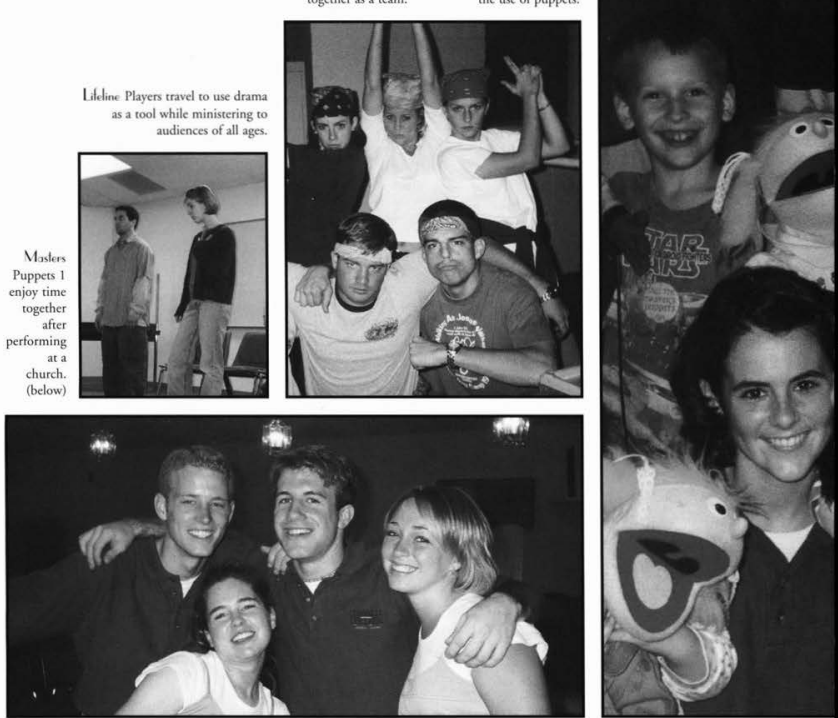

\section{LIFEINE PLAYERS}

The Lifeline Dlayers were a touring drama team that used drama sketches to minister to teens and adults in local churches around the United Stales. The feam performed two programs, cne geared lowards families and ancther geared towards leens. The team traveled throughout the school year, during spring break, and also on an eight-week summer four to the northwestern states.

Row 1: Scott Simons Row 2: Jill Hand, Serh Martin, Jason Tovey, Shana Barba, Amber Wiers

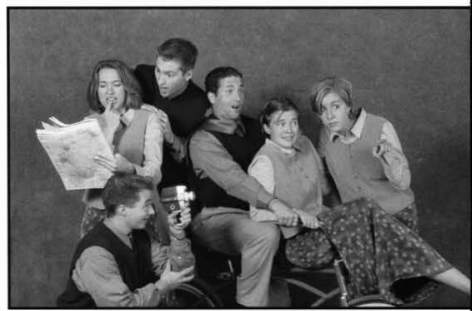




\section{MIS}

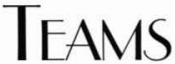

Matt Call, Scott Walker, Jason O'Neal, and Sam

Gilbert raise a flagpole to prepare for a children's program.

\section{ARIZONA}

Row 1: Sarah Greene, Sherri DeWitt, Laurie Rost, Kerri Herrold, Alyssa Rost, Karen Leininger, Geneva Roberts, Christine Trago, Row 2: Jack Bailey, Ken Mansfield, Dale Sorensen, Josh Sherwood, Zack Rhodes

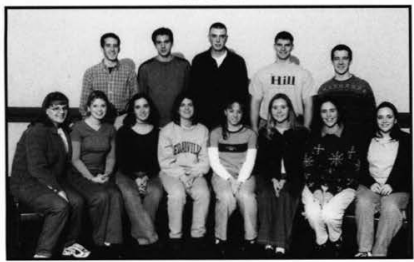

\section{INNER CITY IMPACT}

Row 1: Krista Holmes, Jessica Stevenson, Row 2: Erin Brauns, Kristi Schulter, Michael Pierson, Jordan Parrick, Debbic Huff, Marlena Proper, Row 3: Tiffany NewDay, Emily Bohl, Jessica

Mathias, David Mowrey, John Mason, Ty Waardenburg

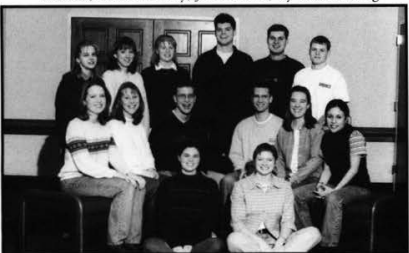

Students dress up as

clowns to entertain Home.

King, and Kristina cleaning to sing Christmas tunes.
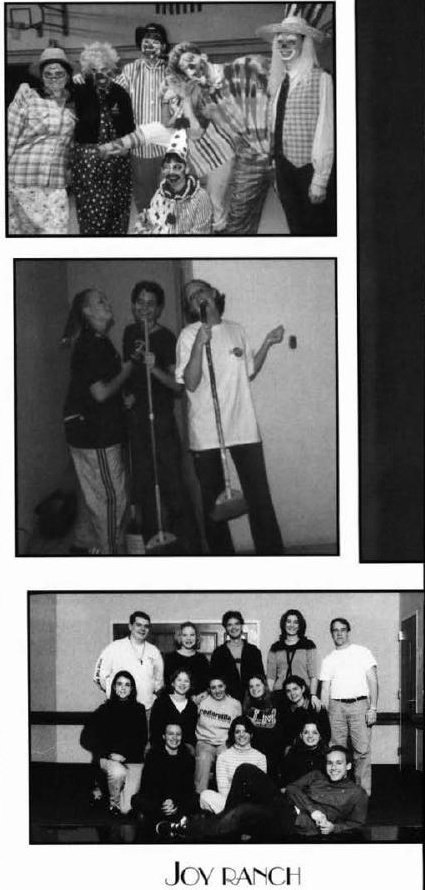

Row 1: Joel Hollins, Row 2: Kristi-Lynn Korczy, Julia Christner, Erin Ewig, Row 3: Missy Lasso, Lindsay Kaiser, Jean Osborne, Amy Harrison, Sarah Pollock, Row 4: Brent Krumdiack, Amanda Taylor, David Perlow, Cheryl Elliott, Tim Cochrell 


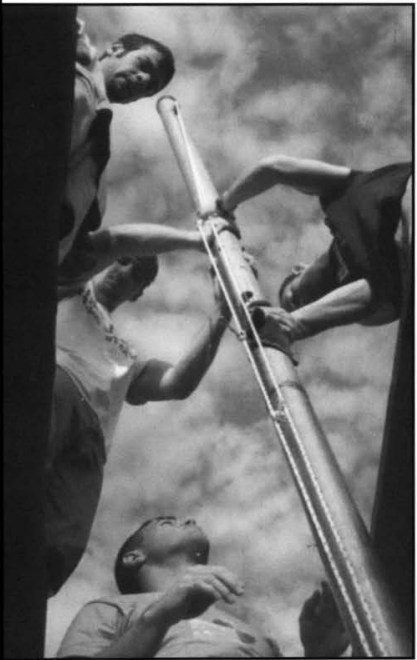

\section{MASTER'S MISSION}

Jenny Elliott, Beth Gosztyla, Gretchen Vuurens, Not Pictured:

Nathan Olin, Eric Pauling, Tiffany Tabor

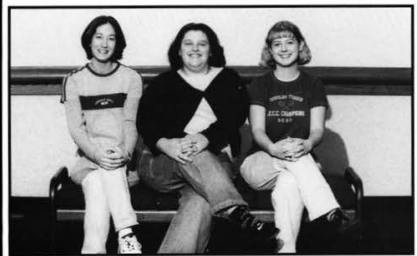

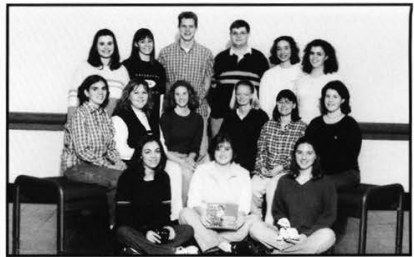

SHEDHERD'S HOME

Row 1: Jennifer Griffith, Elizabeth Klawer, Rachel Schuh, Row 2: Candice Brown, Rachel Frey, Christa Hill, Joanna Carr, Joy Shustack, Jennifer Gration, Row 3: Maranatha Ruberg, Jennifer Ferguson, Nathan Pennington. Tim Seigneur, Rachel Ryan, Rebecca Vitarelli

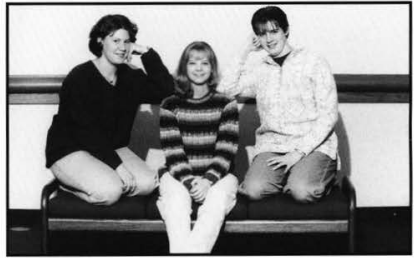

DACIFIC GARDEN MISSION GOSPEL LEAGUE

Amie Bockstahler, Jillian Massey, Elizabeth DeBoet

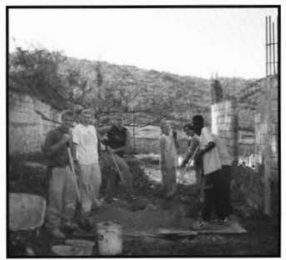

Traveling with MIS teams can be hard work as shown by these students who are mixing concrete. 


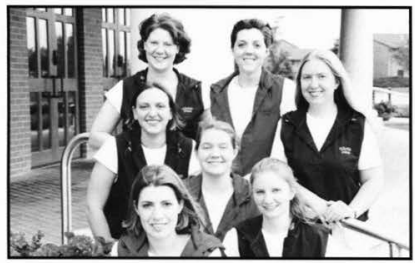

AIBANIA

Row 1: Jennifer Caron, Row 2: Amber Isbell, Beth Cooley, Mary Zuleger, Row 3: Amie Bockstahler, Jewel Alley, Jennifer Davis, Not Pictured: Lynn Koetsier

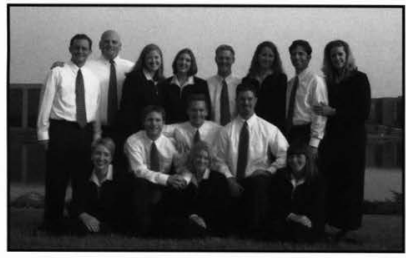

\section{AUSTRAIIA}

Row 1: Holly Kuhn, Jenny Armour, Sheli Oberbeck, Row 2: Benjamin DeLong, Stephen Gaines, Stephen Light, Row 3: Steven Norris, Brett Buckingham, Katherine Masterson, Marcella McCumber, Andrew Hedges, Risha Stockton, Nick Awabdy, Nancy Awabdy

Annie Miller and Jen Larson prepare to lay a layer of cement and tile for missionaries

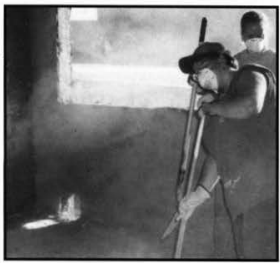

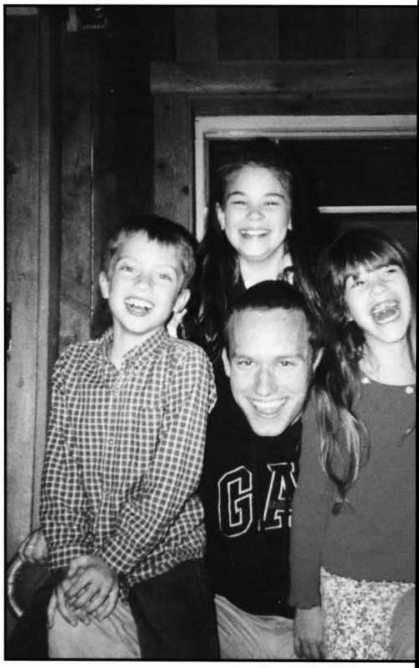

\section{COSTA RICA}

Row 1: Stephanic Aittama, Row 2: Ruth Wagner, Kacie Hayes, Erika Bodden, Rachel Halleland, Row 3: Genoveva Chipe, Jenny

Stryker

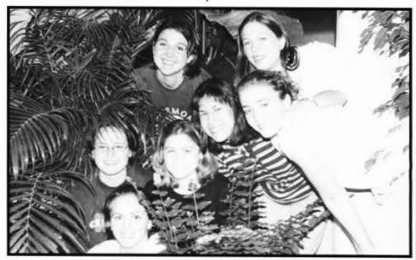


Matt Call entertains a child while on lunch break in Jamaica(right).

Megan Peterson, Becky Kyle, and Julie Finnigan spend time with some children in Jamaica.

Members of Master's Mission 2000 find time to relax during their missions trip (below, right).

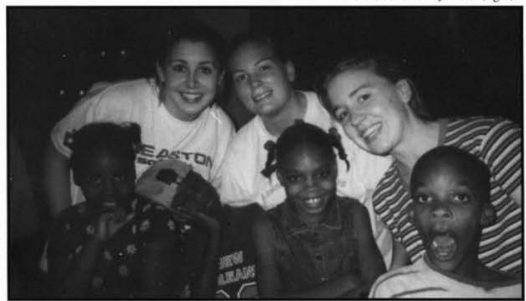

\section{ITAIV}

Row 1: Marisa Flory, Stephanie Kreuder-Crowell, Eric Pauling, Kimberly Wills, Row 2: Daniel Hankinson, Melissa Bielo, Christine Davlantes, Roxy Huck, Scott Huck

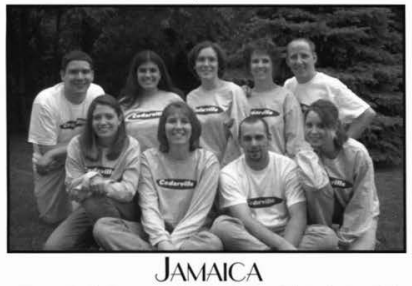

Row 1: Becky Summers, Alicia Good, Matt Call, Becky Campbell,

Row 2: Annie Miller, Jen Larson, Julie Winar, Karen King, Jen Grubert, Theresa Tallarita, Row 3: Nick Collins, Sam Rives, Kristina Parr, Sam Gilbert, Julie Finnigan, Dan Hacker, Row 4; Becky Kyle,

Scott Walker, Micah Mitchell, Megan Petersen, Jason O'Neal
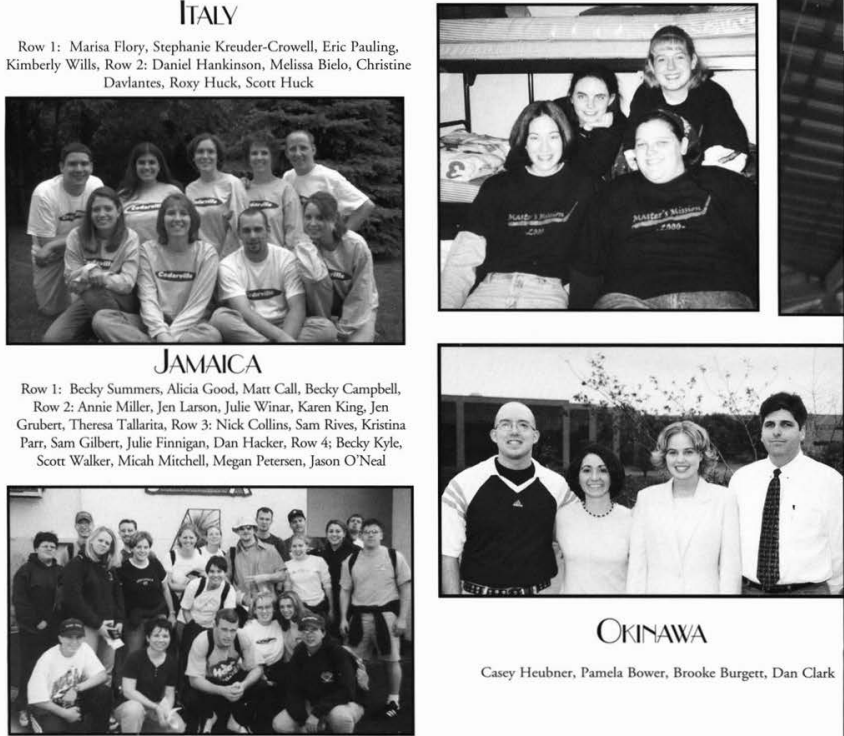

\section{Orinawa}

Casey Heubner, Pamela Bower, Brooke Burgett, Dan Clark 


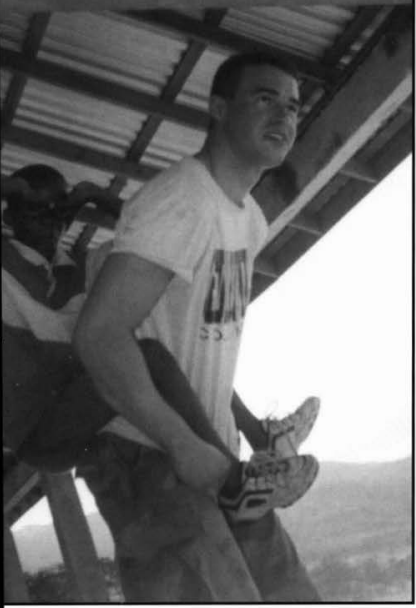

\section{SOUTH AFRICA}

Row 1: Stephen Zarrilli, Rebekah Reynolds, Matthew Tucker, Row

2: Amy Reed, Taira Zgrablich, Kirsten Gibbs, Benjamin Dunn

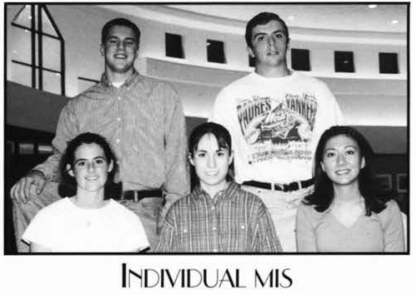

Row 1: Casey Platt, Jen Lutes, Lina Ponder, Row 2: Andrew Gingrich, Benjamin Saturley

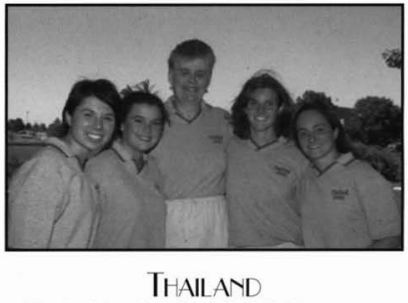

Alicia Good, Amanda Byrd, Judi Shrubsole, Kristen DeVinney, Fernanda Fernandes
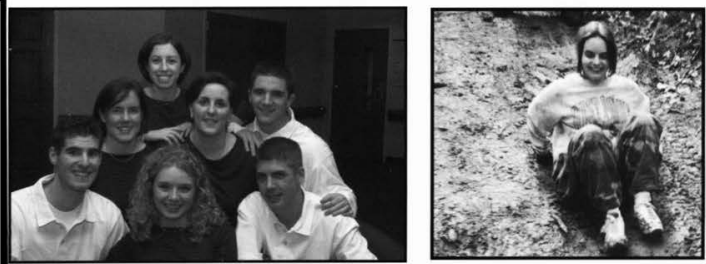

Being on a missions trip brings about unique opportunitics such as sliding down a hill of mud. 
For you were once darkness, but now you are light in the Lord. . . 


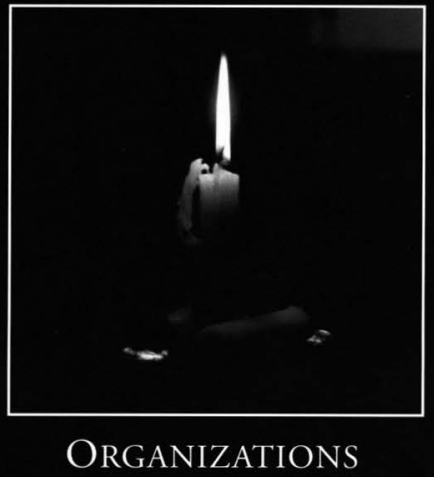




\section{AITP}

AITP provided MIS and

CIS majors with

opportunities to learn about technology through seminars and projects. They hosted a speaker from the technology community each Quarter. They also had monthly meetings and developed web pages.

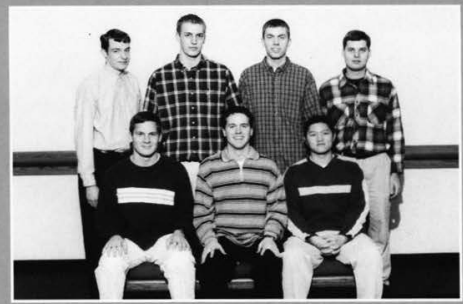

Row1: Chancy Persons, Ben Nelson, Phil Chuang Row 2: Jason Hohercz, Jonathan Cunningham, Andy Kempe, John Mason

\section{Alpha Beta PHI}

Alpha Beta Phi was a national theatre organization that worked to promote theatre awareness. To accompish this, they invited guest professional artists to perform on campus throughout the year.

Row1: Clarissa Band, Vanessa Baker, Laura Livingston, Jessica Wagner Row 2: Greg Davis, Jason Pierson, Brendon Christofer, Jeremy Ryan Brown, Jeremy Pierre, Don Miner

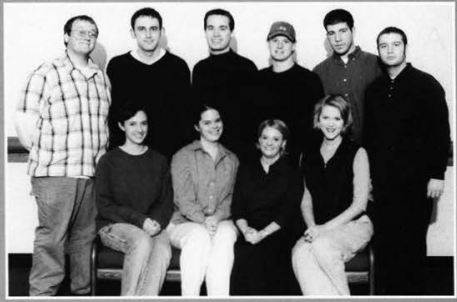

Row 1: Delton Hochstedler, Spencer Phelps. Tim Phipps, John Mason, Joel Cramer; Chris Kelleher, Ben Keener, Nate Pennington Row 2: Michal Andras, Marthew Straley, Randall Bouwerns, Neilo Vitali, Joe Ciccanti, David Frerz Joshus Hynes. Dave Marshall, Jod Taylor Row 3: Chris Rishcill. Tim Seigneur, Tim Cochrell, Joel B. Adams, Chris Hutchinson. William Shetron. Ben Saturlcy, Jason Elwell Not Pictured: Drew Beach, Jason Holland, Andy Krum, Ben Lyons, Mike Marsh. Advisor-Dr. Mark McClain. Ken Shierman

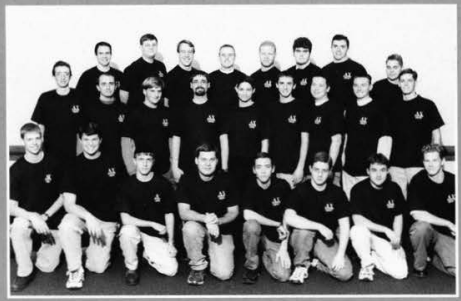

\section{AlPhA CHI}

Alpha Chi was a men's service and discipleship organization that strove to develop leadership. personality, and servanthood in their members. Members were involved in individual and group service projects, with both the college family and community. They were best known for their annual Talent Show. 


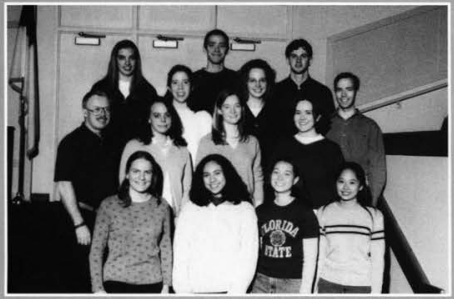

\section{BARA}

BARA was an organization for students who appreciated and participated in the visual arts. They visited studios of artists in the surrounding community and invited them to campus for special presentations. One of the highlights of the year was the studentsponsored art show, where the college family was invited to an exhibit of the club members' own art.

Row 1: Melissa Friesen, Melissa Reyes, Aimee Arimura, Jessica Mao Row 2: Ron Bader, Michelle Acterman, Ariel Starbuck, Sarah Mayer Row 3: Jennifer Holmes, Mandy McLaughlin, Joel Cramer, Sandy Bennett, Jim Llotd, Royce Hunt

\section{$\mathrm{CAB}$}

$C A B$ was an organization devoted to the planning and coordination of many campus events. They were led by Dick Walker, their beloved "Chief." CAB members worked at events such as Getting Started, Little Sibs Weekend, and campus concerts.

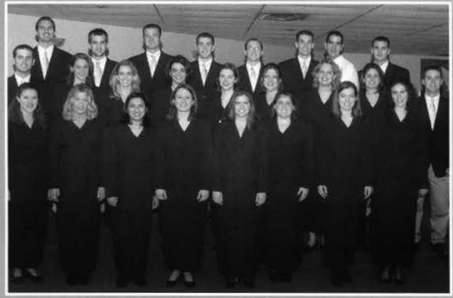

Row 1: Andrea Endicotr. Aimec Nash, Rachel Pucketr, Rebecca Nasman. Nikki Thomas, Sarah Schlicher. Molly Rexford, Geneva Roberts Row 2: Robert Lutz, Sarah High, Shawn Stephens, Maren Meyers, Christy Heflin, Kandace Kenyon. Mandy Hlad, Rebecca Leatherman, Ben Nelson Row 3: Grady Peeler, Caleb Smith, Scott Walker, Michael Reed, Gabe Schlappi, Adam Rizer, Paul Gwilt, Michael Call Not Pictured: Kara Doden, Amie Poelman, Lisa Katz

\section{CCEMS}

CCEMS was the first collegiate EMS service in the U.S. They operated a fully equipped ambulance available 24 hours a day, which covered the Cedarville College campus and assisted with the area township. CCEMS was a volunteer student-run organization with EMT basics. Intermediates, and Paramedics. They strove to minister to students by meeting both physical and spiritual needs.

Row 1: Travis Hermann, Michael Keedy, Josh Tramp, Row 2: Elizabeth de Boer, Biam Davis, Jason Wendzel, Mark Collins Row 3: John Lucas,

Alicia Ayers, Justin Wood Not Picrured: Deb McDonald, RN, Dave Carr, Jim Billock, Kyle Miller

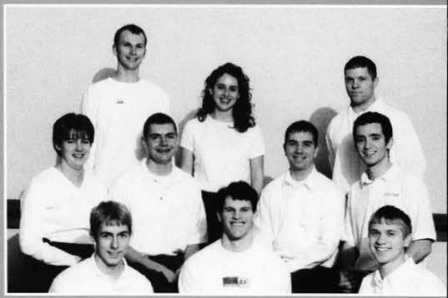




\section{Cedars}

The purpose of Cedars was to inform, enlighten, and inspire. They strove to provide the Cedarville College family with, "all the news that was Cedarfriendly."

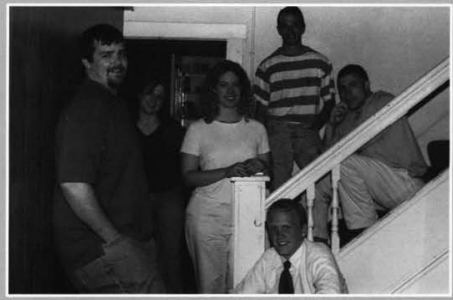

Row I: Brandon Perkins

Row 2: Aaron Warriner.

Carol Marks, Gudron

Olson, Nate Jenkins.

Pete Bednarek

\section{Chi Delta Efsilon}

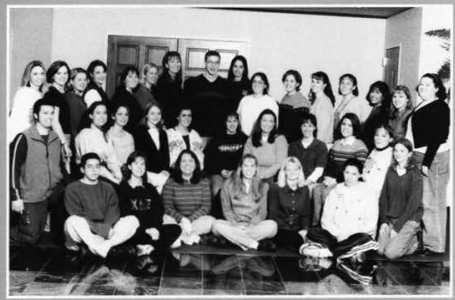

Chi Delta Epsilon was a professional organization in the area of education with an emphasis in service and professional awareness.

Row 1: Willy Bill, Julie Trout, Lisa Smith, Krisin Scherloh, Joanna Carr, Leanne Heath Row 2: Michael Holden. Erin Lec. Kim Wills, Jennifer Weaver, Rebecca Vitarelli, Parricia Hershberger, Jillima Cross, Daniclle Hatfield, Bethany Tate. Hannah Dager, Mary Zuleger Row 3: Christine Olbrick. Julic Hick, Shelley Hanson, Megan Mate, Heidi Yoder, Becca Jenson, Stephanie Kreuder-Crowell, Michael Pierson, Karen Mapes, Sarah Schlicher, Julie Winar, Chrisina Wheatley, Christine Davlontes, Erika Bodden, Amber Coldwell, Beth Gocyryla

Row 1: Joy Carl, Julie Quinn, Nathan Childers, Teri Doleys, Amy Cliffe, Jennifer Holmes Row 2 . Jordan Patrick, Kristin Newman, Kristen Keller, Casey Platt, Karl Feucht Row 3: Kristin Morris, Susan Plummer, Andy Gingrich, Dareen Fuhr, Rachel Bontrager, David Malwiz, John Sorrell. William Worring, Mike Call, Michael Pierson, Erin Burkhardt Row 5: Peter Blodgetr Not Pictured: Bethany Hoff, Alicia Cook

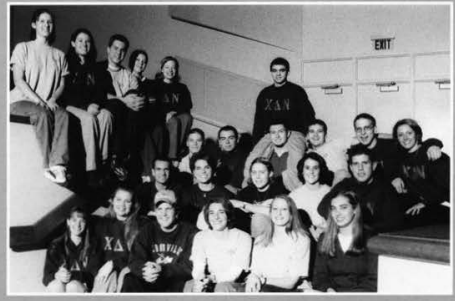

ChI Delta $\mathrm{Nu}$

Chi Delta Nu existed to provide opportunities for students to express their passion for youth ministry. Their goal was to be involved in God's work. especially in the area of youth ministry. One of their key events this year was CedarMania. 
ORGANIZATIONS attempt to recruit new members each year through booths and refreshments.
A group of students show their school spirit while performing the limbo at SGA's annual Hoopla.
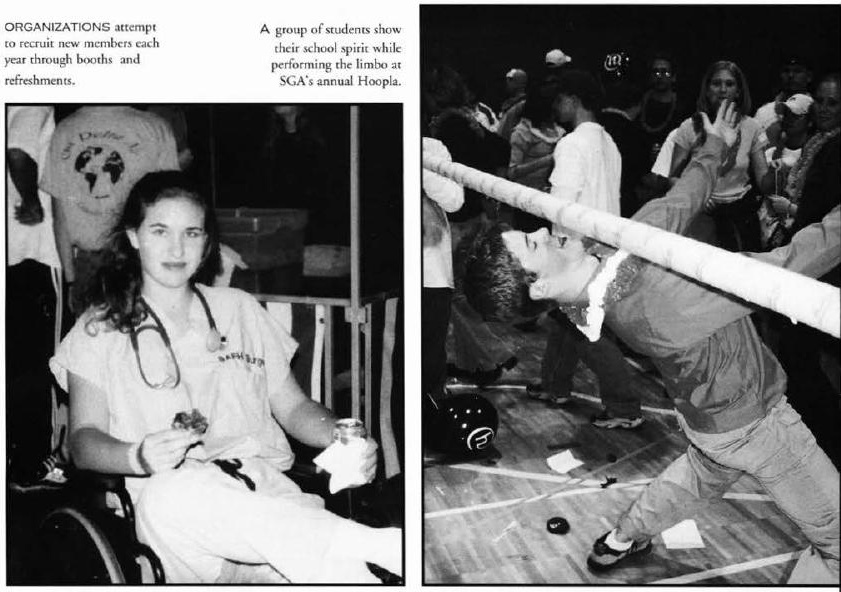

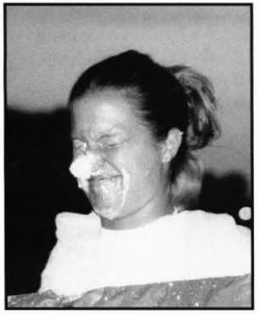

STUDENTS show their support in the organizational fair by allowing pies to be thrown in their faces.

SPL members Jon Faullanes, Grady Peeler, and Eric Bender demonstrate the true purpose of all motorcycle riders.

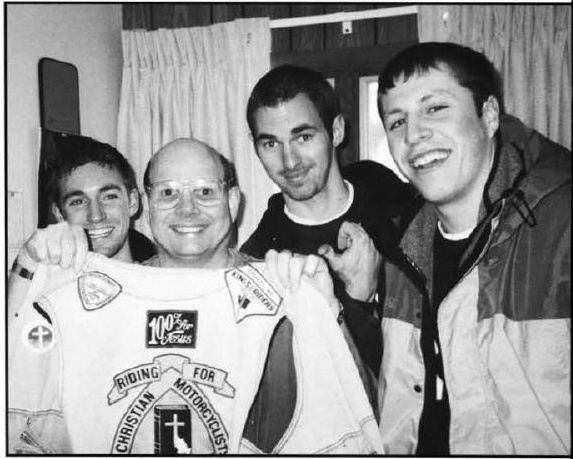




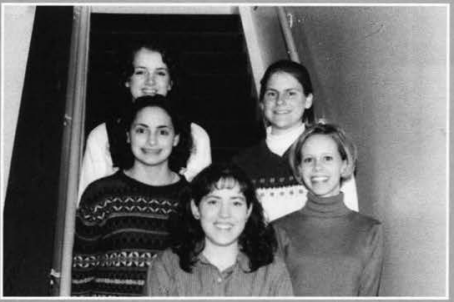

\section{Chi Sigma Iota}

This organization increased the awareness of the information training field through membership in ASID, a professional organization, and through performing one-on one computer training workshops.

Row 1: Tami Wortman Row 2: Amanda Pilgrim, Jennifer Cobb Row 3: Lissa Young, Kelly Reirz

\section{$\mathrm{CHI}$}

\section{THETA PI}

This organization that strove to provide information and opportunities related to ministry in medicine. They did this through speakers. social functions, and field trips. They also provided the science department with equipment for labs

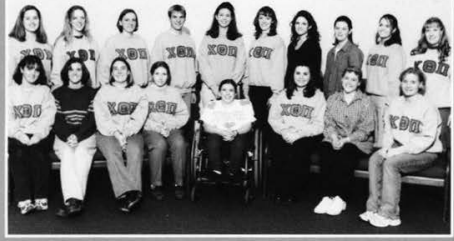

Row 1: Heather Brown, Kristin DeVinney, Lisa Hockenberry, Amanda Lynn Beatrie, Deborah Clingman, Maranatha Ruberg, Heidi

Kinniburgh, Sarah

Daniels

Row 2: Linda

Danncmiller, Sabrina Springer, Amber Isbell, Dana Anglund, Melanie Reber, Michele Blalock, Michelle Moore, Staci Weldon, Krisin Farmer, Kathy Steingass
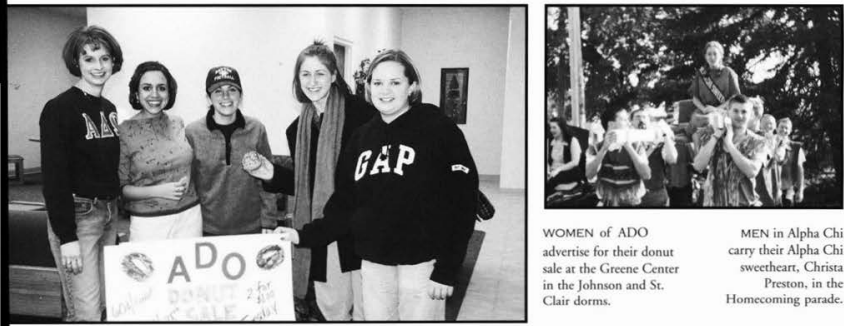

WOMEN of ADO

advertise for their donut sale at the Greene Center in the Johnson and St. Clair dorms.
MEN in Alpha Chi carry their Alpha Chi sweetheart, Christa Preston, in the Homecoming parade. 


\section{Class of 2000 OFFICERS}
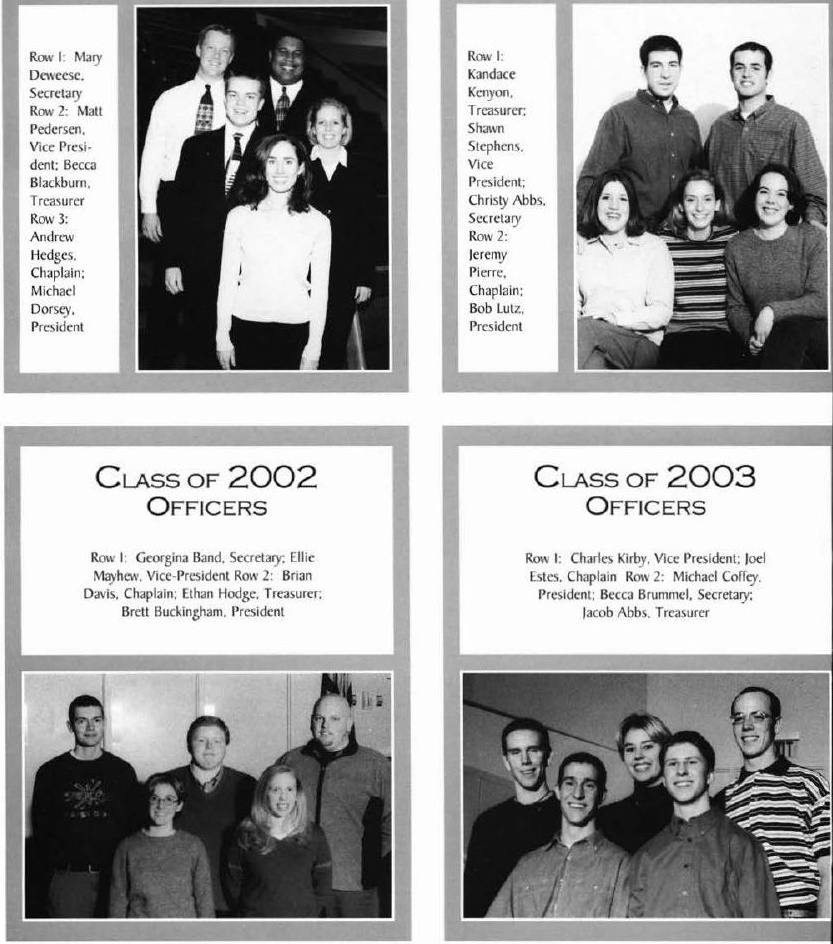
Row 1: Rachel Thompson, Sarah McDonald, Bethany Bishop, Catherine Spink, Rebecea Amsturz. Melamic Brossoit, Amanda Byrd Row 2: Allison Edwards, Bethany Kcilman. Becky Garber, Sarah Tomkinson, Corrie Christiansen, Deanne Butthuis, Fernic Fernandes. Lura Keller, Amie Bockstahler, Heidi Kinniburgh, Kimberly Tipton, Rachel Williams Row 3: Susan Wallace, Emily Gayer. Michelle Moore, Frika Kipp. Sech Stevens, Kristina Bilodeau. Sarah Duda, Tom Lawson, Matt Bicknell, Dan Blosser, Danelle Rocke, Adam Warnken. Julie Maurborn, Beth Mille, Amber Isbell, Carolyn Carlson (Advisot)

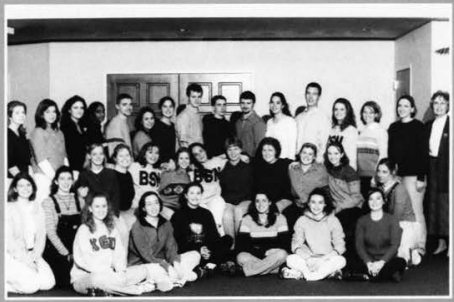

CNA

The Christian Nurses Association provided opportunities for nursing students to serve others, encourage each other. and bring unity among the different classes.

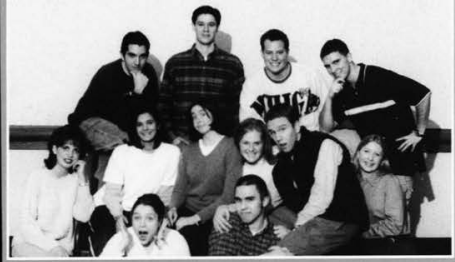

\section{DEBATE}

The debate team was composed of twelve members who worked both individually and as a team. The members studied a wide variety of topics and prepared various defenses on these topics. They competed against students from schools throughout the nation.

Row 1: Michael Coffey, Mark Holden Row 2: Jennifer Hobbs, Karen Belding, Rachel Willimas, Amy Reno, Meg Jenista, Rachel Beach Row 3: Paul Gwilt, Matt Kessler, Henry Bouma, Kevin Hamilton

\section{DELTA CHI}

Delta Chi was an organization for secondary education majors. Delta Chi hosted seminars to help educate their members. Delta Chi's main activity was the Impact Teacher of the Year, which honored two high school teach. ers.

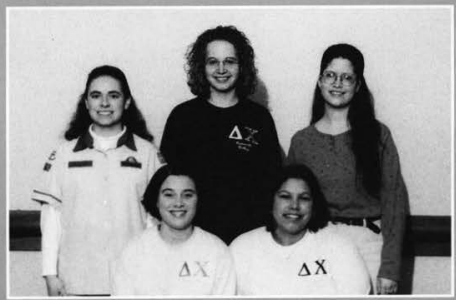

Row 1: Erin Troike, Bethany Hoff Row 2: Kathryn Robers, Sandy Bennett. Angela Rousseau Not Pictured: Keiko Noble, Cindy Myezka, Ryan Roesler 


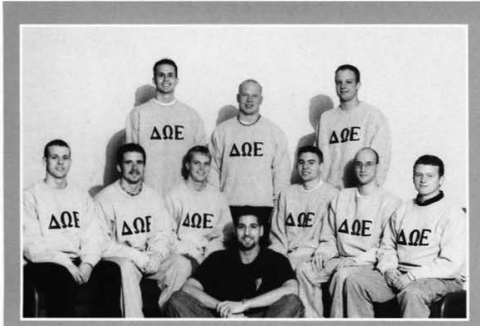

\section{DELTA OMEGA EPSILON}

DOE was a men's service organization dedicated to providing opportunities for commited college men to serve communities surrounding Cedarville. They sponsored the annual Cupid's Bash, which provided a Christian-oriented party for little sibs weekend. They also organized an annual coat drive for the needy in Xenia and Springfield.

Row 1: Nick Schlappi, Lance Ferguson, Christopher Buck, Ted Forrest, Robb Burt, Brian Shook, Jonathan Collins Row 2: Ben Thompson, Jacob Pyles, Jared Coleman Not Pictured: Daniel Price Jr., Tony Ziel

\section{DELTA PI SIGMA}

DPS was a women's service and social organization that focused on meeting the needs of the faculty and staff of Cedarville College as well as providing social activities for the student body.

Row 1: Candace Dodge, Kristi High, Lindsay Peterson, Sara Joy Eleveld, Joanna Houck, Lauric Tait, Sarah Dunn

Row 2: Janc Adams Smith (Advisor), Shanna Lenhart, Lisa Katz, Kathryn Hofert, Suzanne Eaton, Laura O'Donnell, Aamie Guptil, Aimee Nash, Jenny Troyer Not Pictured: Elisabeth Morris, Jessica Terry

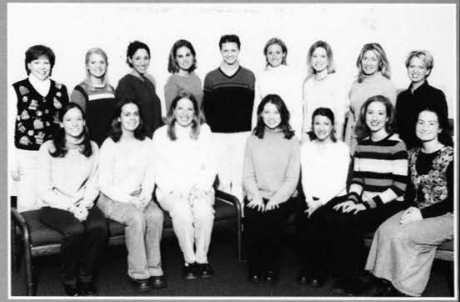

\section{EARTH STEWARD- SHIP}

The purpose of ESO was to promote understanding and appreciation of God's creation in response to their key verse. Genesis 2:15. This year, ESO was involved with leaf-raking events, the Perrin Woods clean up, and recycling projects

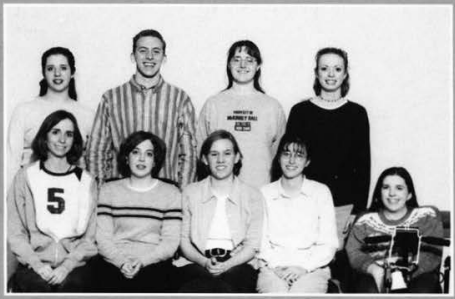

Row 1: Jessica Baratta, Megan Whitman, Katie Zimmerman, Ellen Laber, Deborah Clingman Row 2: Christine Brittin. William Leukhardt, Charis Nims, Amanda Kirchner Not Pictured: Kimberly Eridon 
Row 1: Amanda Melcher, Emily Newton, Sara Wiersma

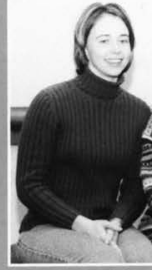

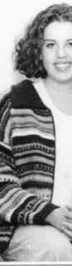

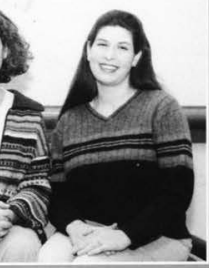

\section{EPSILON}

OMEGA

Epsilon Omega, the social work honor society. promoted achievement in the area of social work by recognizing a social worker from the community, honoring a student majoring in social work. and presenting at a social work conference.

\section{FORENSICS}

The Forensics team utilized their communication skills as they involved themselves in Inter-collegiate speech competitions.

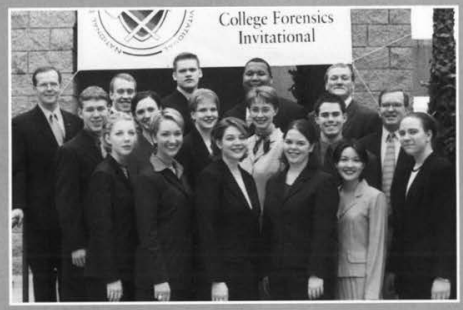

Row 1: Rebecca

Gapinski, Holly Kuhn,

Wendy Williamson,

Vanessa Baker, Ruth

Back, Katherine Witmer

Row 2: Brian Coon,

Clarissa Band, Andrea

Greening, Shelley Yahara,

Philip Sheward, Matt

Moore Row 3: Dr.

Robey, Tim Cochrell,

Justin Swanson, Michael

Dorsey, Greg Davis

\section{HOMES FOR LIFE}

Homes for Life worked with Habitat for Humanity to build homes for people living in unsafe conditions. They used their variety of skilled and less-skilled workers to create Quality homes for support recipients. Homes for Life had a heart for helping those unable to provide Quality housing. They selflessly worked during their weekends to help those home owners.

Rowl: Stephen Matrick, Dannielle Hagen, Dana Davis, Michael Holden Row 2: Erin Knowles, Allison Hume, Christy Abbs,

Kristin DeVinney, Shaelah Weber, Miehelle Corning Melanie Reber, Jeanne Moynihan, Hearher Brown Row 3: Tim Cochrell, Zach Wheeler, Dan Montanye, Jason Smith, Dave Gross, Jacob Abbs, Jonathan Fuge, Willy Bill, Bob Kocher, Kevin Leverson

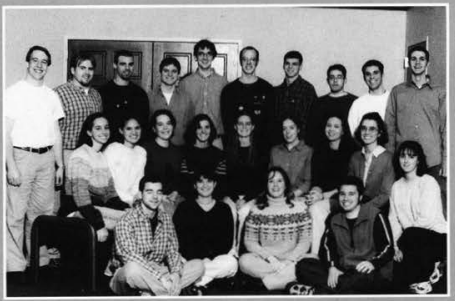




\section{IEEE}

The Cedarville College Student Chapter of the IEEE was an organization committed to the professional development of electrical engineering students. This included providing resources such as scholarships, access to technical publication, and student competitions.

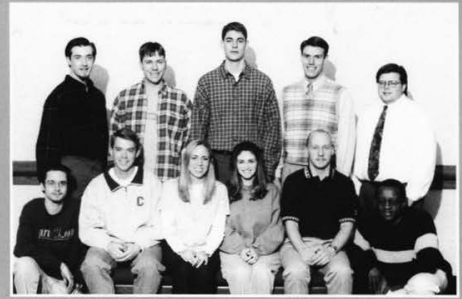

Row 1: Mike Wade, Nelson Lewis, Robyn Dressler, Stacie Bennetr, Bronson Hokuf, Roy Mwangi Row 2: Tim Gilmour, Nathan

Wright, Jesse Hornback. Curtis Sparks, Arik Akerberg

\section{INTERNATIONAL FRIENDSHIPS}

International Friendships had the opportunity to reach out to children from different countries. They were able to build relationships with the children and tell them Bible stories. It was their heart's desire that these children come to the Lord. grow in Him. and, in turn, reach out to others.

Row 1: Mike Kibbe, Erin Weber Row 2: Robert Velazquez, Kristy Mandigo, Charis Nims Row 3: Rebekah Huffman, Roy Mwangi, Joy Carl, Matt Johnson
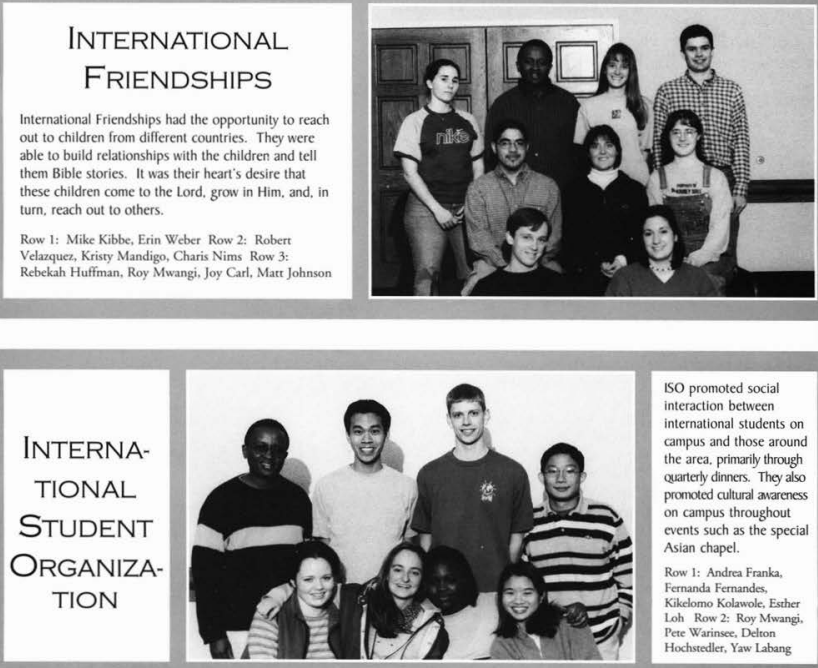

ISO promoted social interaction between international students on campus and those around the area, primarily through Quarterly dinners. They also promoted cultural avareness on campus throughout events such as the special Asian chapel.

Row I: Andrea Franka, Fernanda Fernandes, Kikelomo Kolawole. Esther Loh Row 2; Roy Mwangi, Pete Warinsec, Delton Hochstedler. Yaw Labang 


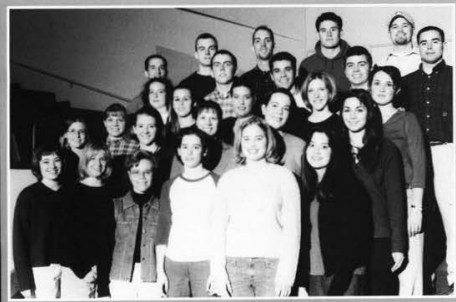

\section{KEA}

KEA was an organization for business majors to learn more about their career fields. It provided opportunities to hear from various speakers. KEA also took part in an annual trip to visit Washington, D.C.

Row 1: Rachelle Denton, Amanda Bartholomew, Lucinda Congdon, Hannah Lakes, Brooke Burgetr, Sharon Bullock Row 2: Katie Koeppen, Mandy Herd, Katic McGunnigal, Christy Abbs, Angie Reninger Row 3: Rebecca Allen, Elizabeth Moore, Kelly Boyd, Lori Wilmer, Sarah Leisure Row 4: Jackie Smich, Dan Dunham, Bob Kocher, Brian Aartsell Row 5: Russ Toms, Matt Dunn. Jared Applegate, Josh Girth, Josh Fetzer, Joe Mulvancy

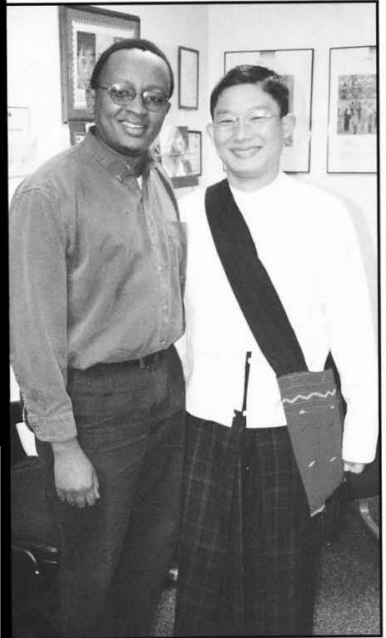

embers of the International Student Organization promote eraction berween international students on campus.
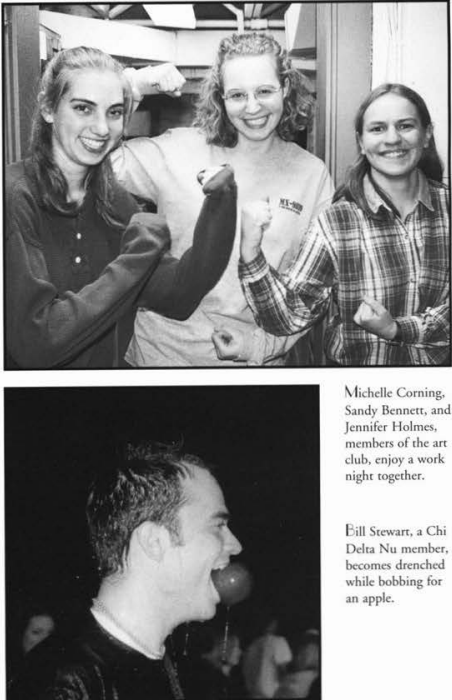

Michelle Corning, Sandy Bennett, and Jennifer Holmes, members of the art club, enjoy a work night togecher.

Bill Stewart, a Chi Delta Nu member, becomes drenched while bobbing for an apple. 


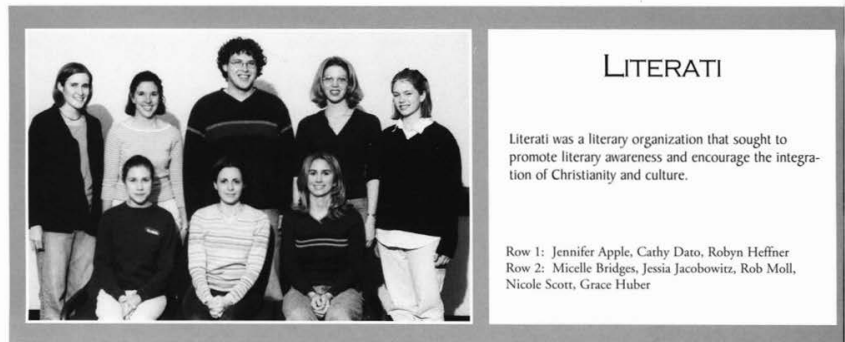

\section{MENC}

MENC was an organization designed specifically for music education majors. Their main goal was professional development. One of the main events of MENC was attending a three-day professional conference for music educators.

Row 1: Hannah Rives, Kathryn Roberts, Phillip Tate, Sarah Murphy, Rachel Schuh, Melanie Slabaugh Row 2: Nikki Thomas, Amy Harrison, Ryan Einfeldt, Tami Ellington, Ken Borror, Jenn Weaver Row 3: Jennifer Drexel, Jennifer Cook, Amy

Lovell, Andrew Williams, Lynette Wagner, Elizabeth Gowdy Row 4: Kim Hain, Janelle Criner, Joseph Mickey, Andrew Nyveldt. Julie Henderson, Whímey Johnson

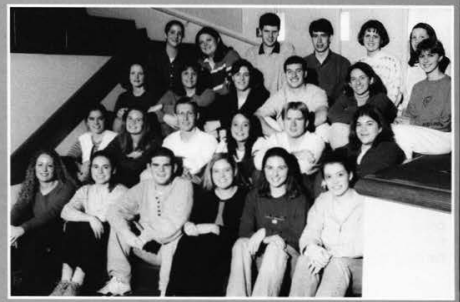

\section{Mu KAPPA}

Mu Kappa was a culturally diverse organization comprised of missionary kids and international students. They were a social organization dedicated to serving the international missions community.

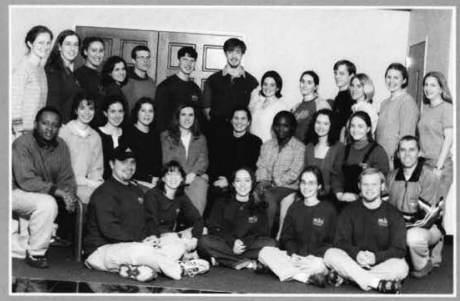

Row 1: Robert Hirschelman, Sracy Hirshelman, Kara Allen, Hannah Allen, Kenneth Waldock Row 2: Roy Mwangi, Laura Cochrane, Heather Roth. Kimberly Yoder. Jayme Brower. Kimberly Ketterer, Kikelomo Kotawote, Katic Jackson, Laura Holzmann, Karl Hjembo Row 3: Rachel Morris, Ann Weeks, Amy Zimmerman, Debbie Buck. Todd Lindler, Jeff Schumacher, Tim Gilmour. Julia Fawcett, Reteckih Huffman, Mike Kibbe, Launie Gallo, Julia DeHarc, Krisci Jonker 


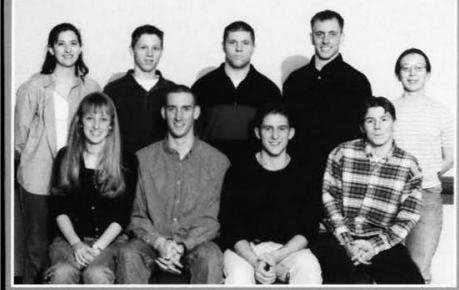

\section{OfFICERS' Christian FELLOWSHIP}

Cadets in Air Force and Army ROTC met for a Bible study called Officers' Christian Fellowship. It was part of a national organization that allowed Christians to contact and fellowship with other Christians at military bases. The college branch focused on ministering to and praying for cadets at other schools and preparing them for unique issues that greet Christians in the military.

Row 1: Ruth Meteer, Jonathan Burson, Joshwa Daniel, Pete Springirth Row 2: Rachel Turkis, Stephen Tidwell, Justin Wood, Tim Sutton. Heidi Kinscy

\section{$\mathrm{PHI}$

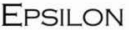

BETA

Phi Epsilon Beta was a service and social organization. The main focus of the organization was to help the Crisis Preg. nancy Center.

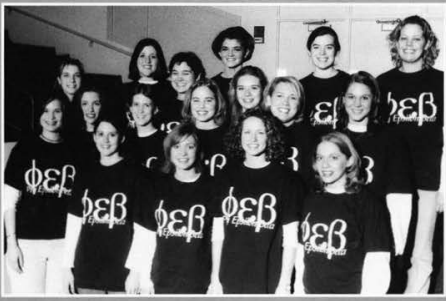

Row 1: Lindsay Borland, Deborah Meizler, Mandy McGuire, Jennifer Drexel, Holly Favorite Row 2: Lindsay Wagner, Lisa MacPhersen, Brooke Burgett, Jenn Grubert, Sarah High Row 3: Jody Thompson, Esther Haffcy, Rebekah Reynolds Row 4: Kandace Kenyon, Suzy Bosworth, Molly Rexford, Mandy Hlad

\section{PhI Gamma PSI}

Phi Gamma Psi was a service and social organization. It allowed women to be involved in a variety of projects that benefited the college and the community. It was their heart's desire to serve the Lord in all that they said and did.

Row 1: Katie Futrell, Melissa Roesch, Lia Davenport Row 2: Risha Stockton, Grace Huber, Jocelyn Jones, Katen Huber, Chris Gideon, Becky Cunningham Row 3: Joy Hester, Bethany Hoff, Kelly Marsh, Shelley Yahara, Mandy Mullen

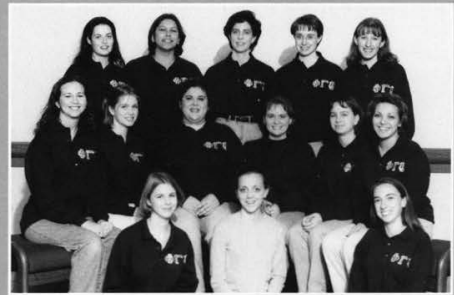




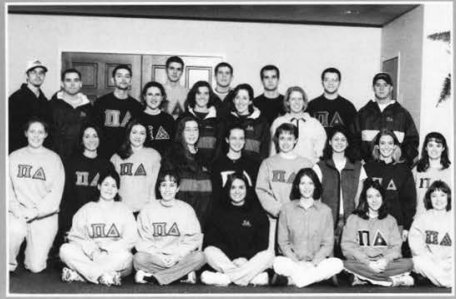

\section{PI DELTA}

Pi Delta was a hospitality organization. They gave tours of the college facilities and shared the excitement of Cedarville College with visiting guests. Christcentered sharing was always a focal point of their work.

Row 1: Jennifer Gration, Laurit Hummitzsch, Tisha Tapp Krista Morris, Kathy Delbu, Rachelle Denton Row 2: Megan Miller, Pam Bower, Bethanne Whaler, Ann Weeks, Erin McDaniel, Shelley Yahara, Rebecca Leatherman. Shawn Stephens, Christina Wheatey Row 3: Andrea Endicotr, Kristin DeVinney, Andrea Parterson, Becca Blackburn Row 4: Mike Wade, Joe Mulvaney, Michael Ferrigno, Kevin Leverson, Karl Feucht, Caleb Smith, Tim Bailev, Jason Arwell

\section{Republican} Club

The college Republicans endeavored to achieve a campus informed about the political world. However, above all, their goal was to understand the role that Christians must take in the political system.

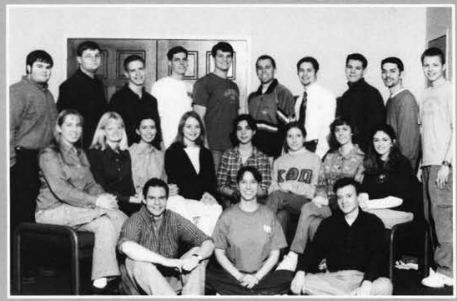

Row 1: Chris Risheill, Kimberly Eridon, Brian Roc Row 2: Kristin Schierloh, Joanna Carr, Amy Reno, Jennifer Weaver, Rebecca

DeSantis, Amanda Lym Beattie, Kristin Bollmeier, Stacie Bennett Row 3: John Higginbotham III, Tim Scigneur, Joel Manuanen, Joel Taylor, Spencer Phelps, Karl Hjembo. Tim Mohler, Benjamin Mohler, Michael Ferrigno, Colby Matthews

\section{ROTC AIR FORCE}

Air Force ROTC was designed to develop future leaders of the Air Force and better citizens for America. Through weekly training, the cadets learned skills essential to leadership and fundamental to the preservation of the nation. Through Air Force ROTC. the cadets were trained to become the influential leaders of America.

Row 1: Jeanette Brogan, Adam Stiegelmeier, Tim Sutton, Joel Roberts, Eric Babson, David Abels, Stephen Tidwell, Ruth Metur Row 2: Matt Snyder, Ben Futoran, Stephen Bruckart, Jonathan J. Burson, Nathan Mann, Andrew Billharte, David Calao, Brandon Dow

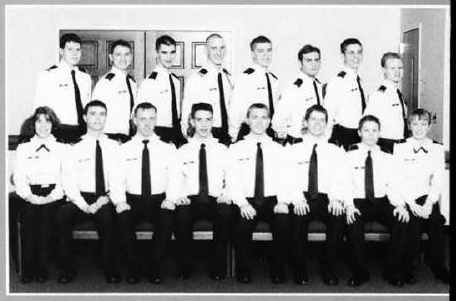




\section{ROTC ARMY}

Army ROTC trained leaders for the 21 st century in preparation for their commissioning as officers in the United States Army. The skills learned both in ROTC and at Cedarville College prepared the cadets for the challenge of presenting the Gospel all over the world.

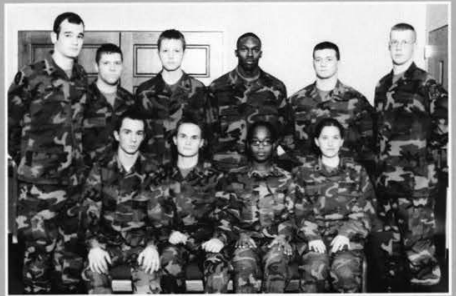

Row 1: Dustin Walker, Levi Gangi, Leah Baker, Rachel Turkis Row 2: Johnny Rough, Justin Wood, Colby Marthews, Micah Hutchins,

Kenneth Nielsen, John Compton

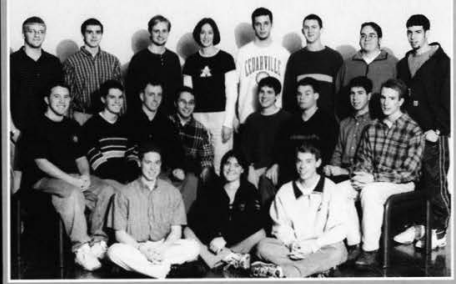

\section{SAE}

SAE was a world-wide organization of professional and student engineers. This organization provided a gateway into the world of engineering. Many Cedarville students had the opportunity to spend time with professional engineers. They also participated in the SAE Congress at the COBO Center in Detroit.

Row 1: Greg Roth, Kristy Mandigo, Nelson Lewis Row 2: David Drye, David Rench, Tim Surton, Nick Tucholski, Richard Brewer, David Wolf, Mark W. Kirby, Greg Reilly Row 3: Jonathan Martson, John Hagley, Kevin Borries, Jenny Elliort, Tim Stoufer. Aaron Roth. Ben Carpenter, Jesse Magnuson
Row I: leremy Bouma, Kelsey Perkins, Matt Call Row 2: Scolt Lehr Row 3: Amy Nehius, Andy Blodget. The Bee, Ben Mohler. Shanna Lenhart. Liz Morris

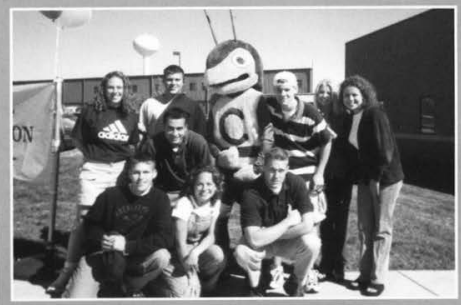

\section{SGA Officers}

The SGA officers served the student body as their elected, student-led governement, representing the needs of all Cedarville students and eliciting change on Cedarville's campus. 


\section{SIFE}

Students in Free Enterprise was a national organization involved on campus and in the community. They strove to promote the notion of free enterprise and glorify God by doing everything in an excellent manner.

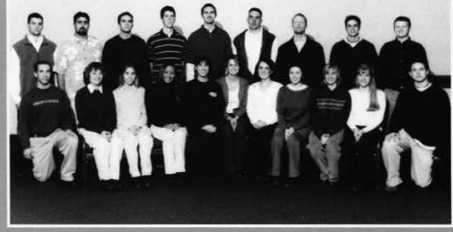

Row 1: Russ Tones,

Rachelle Denton, Danelle

Ellis, Darcy Fivek, Kimberly

Eridon, Lori Bunger. Sarah

Leisure, Jill Mistak.

Amanda Bartholomew,

Rebecca Allen, Andrew

Cheney. Row 2: Chad Fires.

Chad Heise, Justin

Epperhy. Aaron Fourman,

Grady Peeler, Iohn

Hoekstra, Matthew Byrd.

Joseph Sanders, Iohanthan

Collins

KEA members, Josh Fetzer and

Lori Wilmer, make cotton

candy at the orgazational fair.
BRIAN Neizes watches as a student is about to swallow a live goldfish.
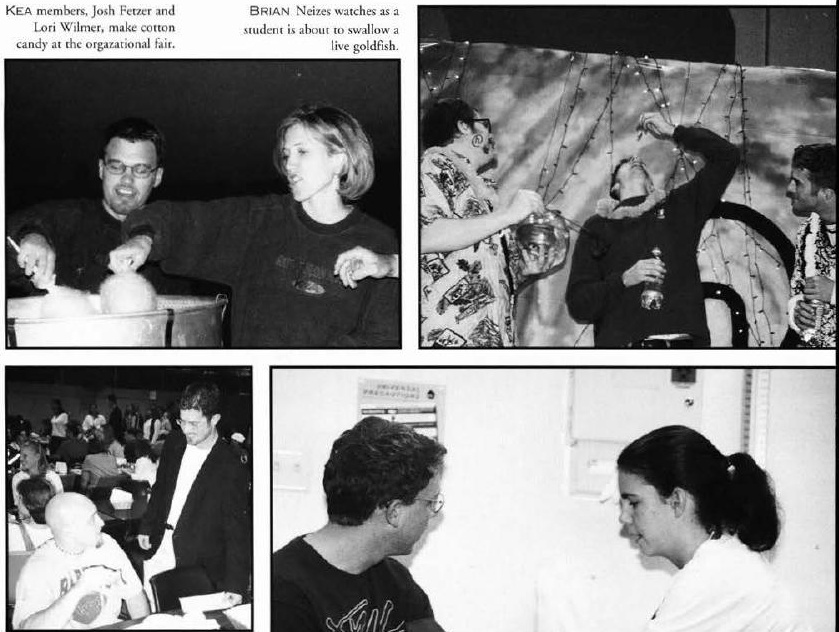

CHUCK's serves as a great place for interaction among students.
JENNIFER Gordon,

a CNA member, takes another student's blood pressure.

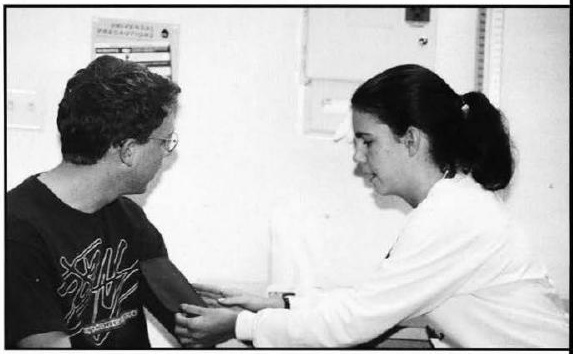




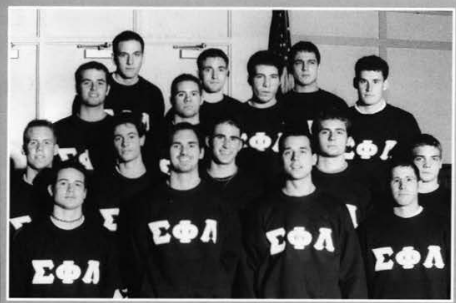

\section{Sigma PHI LAMBDA}

SPL was a social service organization that sought to create unity among its members. Its goal was to serve the campus and community while building male leaders. In addition. SPL worked to develop a bond of brotherhood that would last for years.

Row 1: Andrew Cheyney, Grady Peeler, David Harrison, Roger Swigart, Row 2: Justin Geer, Steve Black, Brock Weston, Zyan Newsome, Matthew Minier Row 3: Bob Lutz, Michael Tharp, Jeremy Pierre, Josh Girth Row 4: Ian Dickey, Eric Bender, Tim Prusha, Nick Spaulding

\section{STC}

STC was a professional organization for Techni$\mathrm{cal}$ and Professional Communication majors. The Cedarville College chapter of STC received a chapter achievement award. During the spring, students traveled to the 47th Annual Conference in Orlando. Florida.

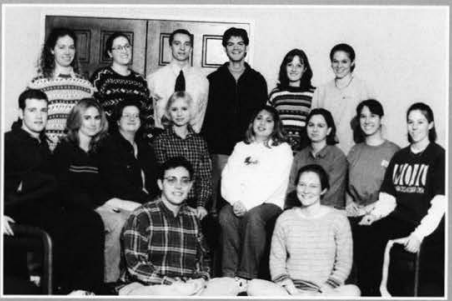

Row 1: James Deaton, Rachel Morris Row 2: Stephen McMurray, Robyn Heffner, Mclanic Scheerchmidt, Aleesha Hager, Jen Castellani, Chris Gideon, Kimberly Eridon, Cascy Ruffin Row 3: Gudrun Olon, Jenn Wallace, Jason Overturf, David Perlow, Sarah Flenar, Sarah High
Row 1: Carolyn Sprankle, Michelle Corning, Melissa Reyes Row 2: Charin Nims, Monica Thomas, Jenn Willis

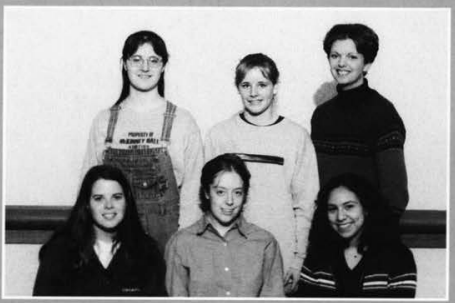

\section{SPANISH \\ Club}

The Spanish Club met together to practice speaking the language and learn more about the Spanish culture. 


\section{STUDENTS FOR RELIGIOUS FREEDOM}

The Students for Religious Freedom promoted awareness on and off campus about religious persecution. They collected blankets to send to the persecuted people in southern Sudan. They also held an underground church simulation to imitate what it would be like to attend a worship service in a persecuted country.

Row 1: Kaley Johnson, Lisa Hockenberry, Amiec Arimura, Angela Rosseau Row 2: Francis Shrubsole, Joel Cramer, Mark Kordic, Tim Gilmor, Benjamin Britton, Ben Nesbitt

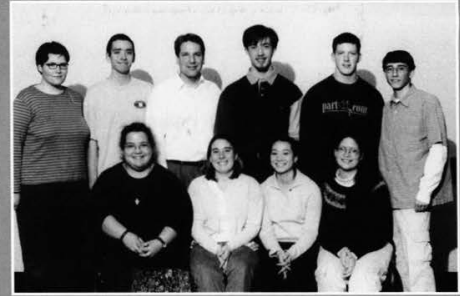

\section{SWE}

SWE was a group that supported and encouraged women in engineering. They had group outings and meetings to provide an outlet to the pressures and stresses that academic life can bring.

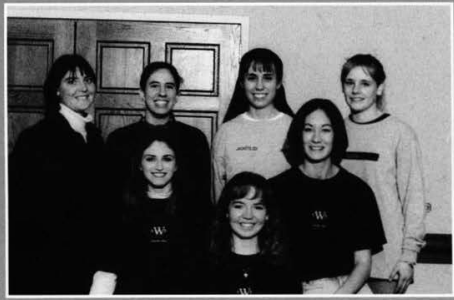

Row 1: Stacie Bennett, Amy Morse, Jenny Elliott Row 2: Kristy Mandigo, Jennifer Chmielewski, Jennifer Lutes, Monica Thomas

\section{TAu Beta Alpha}

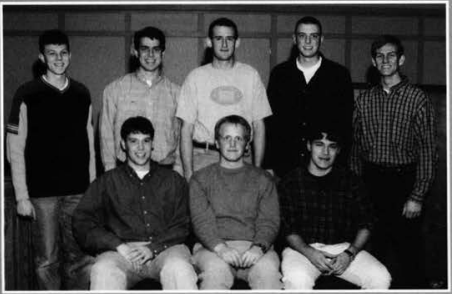

Tau Beta Alpha honored junior and senior engineering students who had achieved academic excellence and demonstrated exemplary character. Under the leadership of their faculty advisor. Dr. Hardy Hegna, they participated in service projects such as mentoring freshman engineering students.

Row 1: Matther Cook, Brandon Perkins (Vice President), Jeff Olson Row 2: John Diggle, Derek Chandler, Lon Noly, Dale Sorenson, Chris Recktenwald (President) Not Pictured: Heather Maybury, Jeff Schumacher, Mike Brame Faculty Advisor; Dr. Hardy Hegna 
Row 1: Chris Calo, Heather Westbrook, Patricia Hershberga, Rachel Heffield Row 2: Allison Reemtsma, Kathy Stcingass, Bronson Hokuf, Sabrina Springer Row 3: Brock Weston, Katic Masterson,

Kimberly Eridon, Sarah Posegate, Benjamin Britton

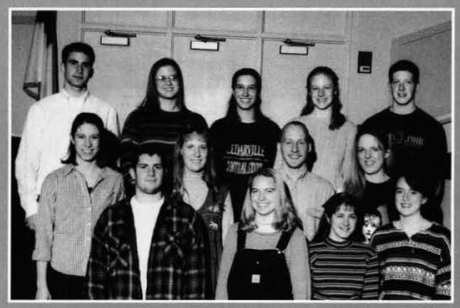

\section{TAU \\ DELTA \\ Kappa}

Tau Delta Kappa provided an opportunity for fellowship. exange of ideas, and support for those students enrolled in the Honors Program.

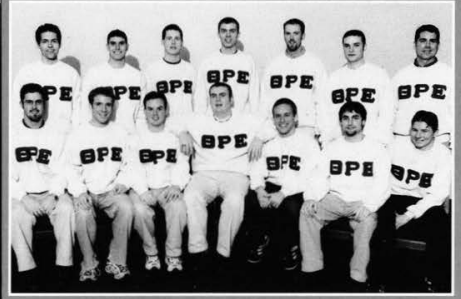

\section{THETA RHO EPSILON}

Theta Rho Epsilon was a men's organization dedicated to following the verse, Proverbs 27:17: "As iron sharpens iron, so one man sharpens another." They accomplished that through activities, accountability. and service. They also strove to see growth in their relationship with God through small group accountability.

Row 1: Steve Light. Nate Lasso, Justin Dyer, Nicholas Powell, Joel Hollins, Mark Clark, Pete Springirth Row 2: Jeremy Bouma. Aaron Habegger, Ryan Cook, Andy Griffith, Tracy Commons, Ben Vlug, Jeff Beste (Advisor)

\section{TIMILATHIIANS}

The Timalathians promoted philosophical discussion on campus through faculty presentations, student forums, and intercollegiate activities. They explored Christian involvement in society in a variety of ways, recognizing that they were called to interact meaningfully with culture.

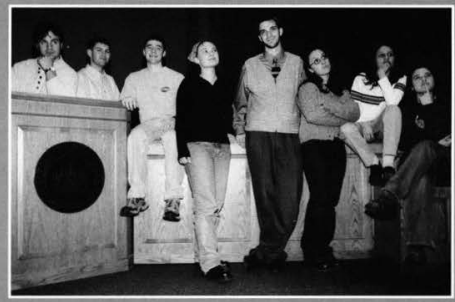

Row 1: Shawn Graves, Michael Essington, Scott Camerson, Ercia Landenberger, David MeGrew, Chrisina Hocevar. Marlena Proper, Erin Troike 


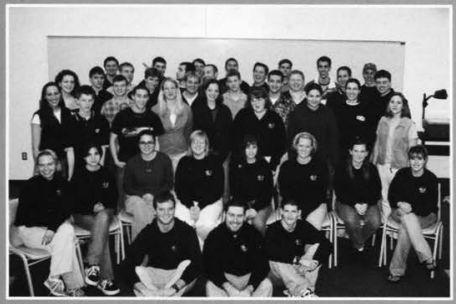

\section{U99.5}

U99.5 was the campus radio station that served the students of the college.

They provided programs daily. featuring music, special events, and live interviews. They also hosted contests for listeners to win prizes, such as gift certificates, tickets, and music CD's.

\section{UAA}

The purpose of the Undergraduate Alumni Association was to serve the alumni of Cedarville College.

Row 1: Erika Kipp, Amanda Oeftinger, Benjamin Mohler, Rebekah Green, Karl Feucht, Justin Geer, Jodi Hurlow Row 2: Jennifer Wishart, Lucinda Congdon.

Rebecca Leatherman. Amy Nyhuis, Shawn Stephens.

Rachelle Denton, Joanna Houch, Alison Reemisma, Jennifer Gration, Joe Mulvaney Row 3: Joel Manwannen, Rejeana Wright, Betsy Linnell, Scott

Simons, Andy Hedges, David Mowry, Scorr Walker, Emily McQuinn, Darcy Fivek, Maren Meyers, Jenn Willis
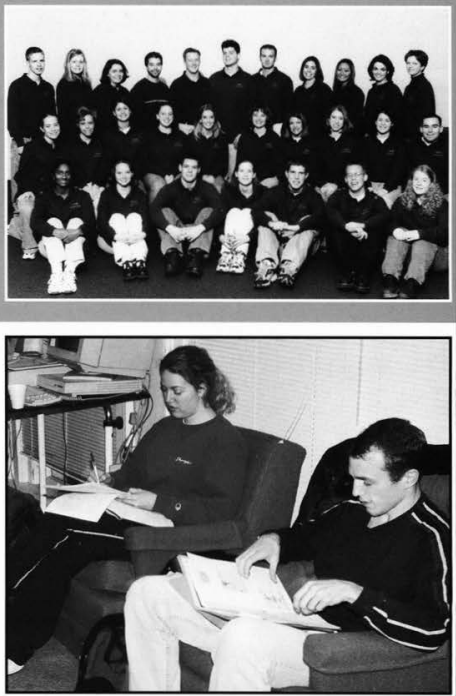

Goodrun Olson and Nate Jenkins proof pages while working to complete this

week's edition.

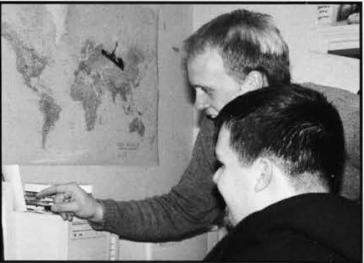

Brandon Perkins and Aaron Warriner, both cditors for Cedars, work hatd to finish an issue. 


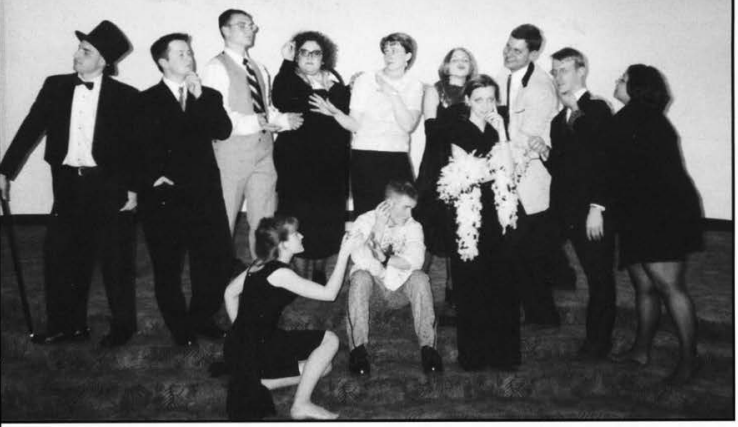

Members of Phi Gamma Psi and Alpha Chi show their acting abilities at their Masquerade.

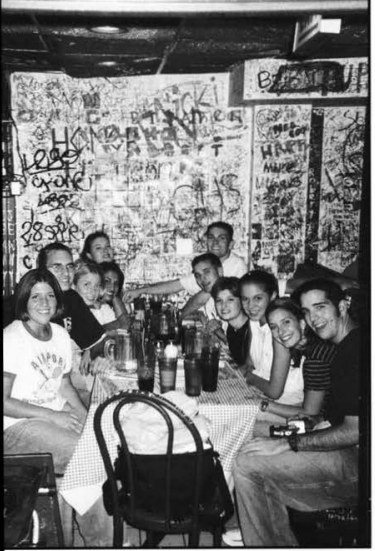

$A B$ members eat out at a pizza hangout. Gino's, while traveling fether in Chicago.
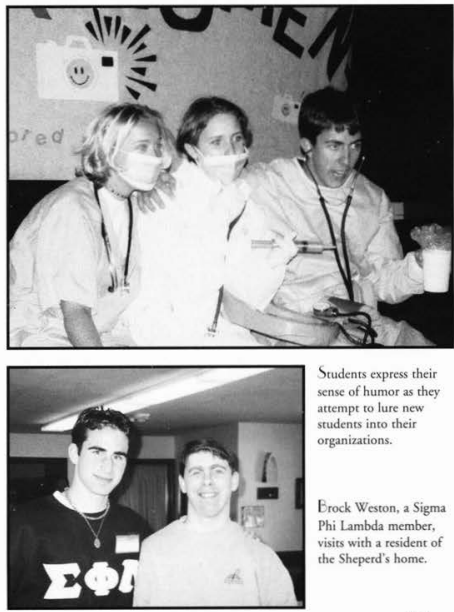

Students express their sense of humor as they attempt to lure new students into their organizations.

Brock Weston, a Sigma Phi Lambda member, visits with a resident of the Sheperd's home. 
... live as children of light, for the fruit of the light consists in all goodness, righteousness, and truth.... 


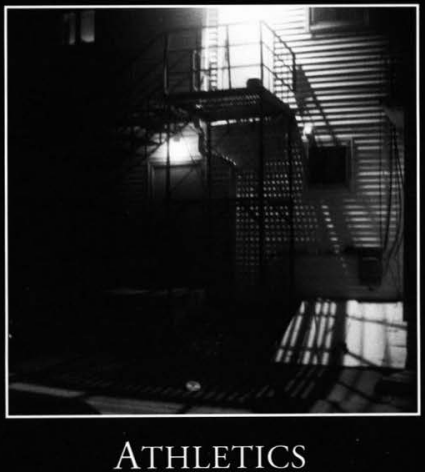




\section{success despite adversity}

The 1999 Yellow Jackets women's volleyball team had an interesting season. They lost three seniors from the 1998 squad. All three, Suzanne Lehmen, Julie Mclntyre, and Julie Opperman were all-conference players. Despite these losses, there was a solid core of returning players to help build up the team. Some of these players were sophomore Heather van $\operatorname{der} \mathrm{Aa}$, junior Lori Bunger, junior Leah Ziegenfuss, and sophomore Amy Martin.

The Lady Jackets looked to team captains, Lori Bunger and senior Pam Huls for leadership. The squad this year was a young team but had the ability to im-

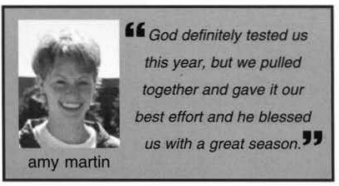

Heather van der Aa had an outstanding season. She led the team in blocks with 146 for the year. Leah Ziegenfuss also had a great season; she had the team high total in kills with 495. Lori Bunger led the team not only with her leadership and example, but also in serving percentage with $98.9 \%$.

The women faced a year full of struggles. There were many hurdles they had to overcome. First, they could not start practices in their own gym for the first few weeks. Injuries and various other adversities plagued the team throughout the seasonb but through it prove. Coach TeresaClark wasconfident in them. They gained all the Lady Jackets were able to keep their eyes valuable experience this year, which will greatly help them be focused on the Lord, remain optimistic, and do their a stronger team next year best.

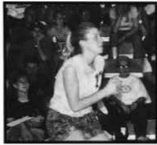

pam Huls cagerly awaits the chance for a hit.

receiving the serve,

Cheryl Meyer start the Jacket's offense.

\section{players}

- row 1: Lachelle

Peters, Casey

Schmidt, Courtney Williams, Cheryl

Meyer, Assistant

Coach Suzanne

Lohman.

- row 2: Chrissie

Fretts, Alison

Reemtsma, Pam

Huls, Lori Bunger

- row 3 . Head

Coach Teresa Clark

Amy Martin, Leah

Zieganfuss, Heather van der Aa. Jennifer

Tetrick, Sarah

Oleszczuk, Assistant

Coach Jim Clark
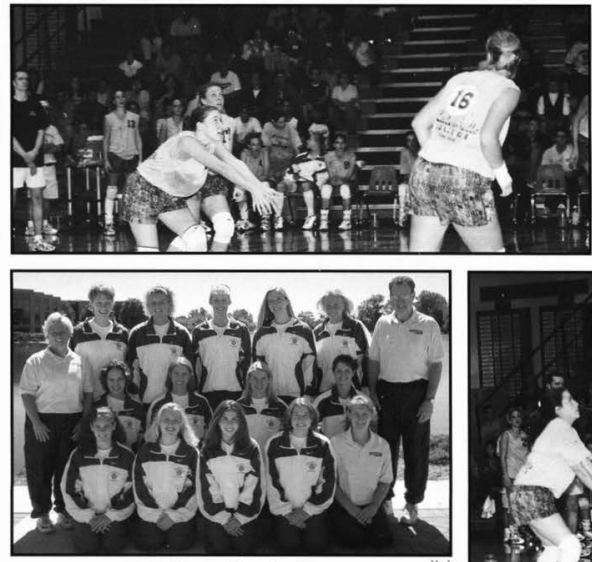

women's volleyball team

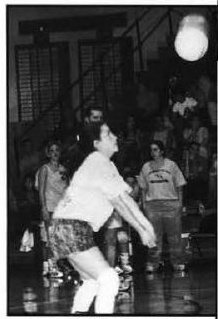


senior keeper

Katie Mummau

clears the ball

from the box.

\section{stats}

- The women's soccer team finished 5-9-0 overall.

- The team comleted the season with a 3-5-0 in the American Midwest Conference.

\section{stars}

- Michelle Rulhman led the team with eight goals and six assists. - Kristin DeVinney has started every single match since the beginning of the program in 1997.

\section{scores}

\section{CEDARVILLE}

- Saint Vincent

- Otterbein

- Taylor

- Marietta

- Geneva

- Urbana

- Seton Hill

- Notre Dame OH

- Walsh

- Wittenberg

- Marion

- Malone

- Denison

- Tiffin
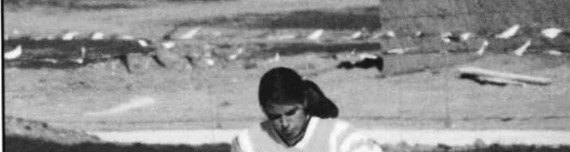

kristin DeVinney

outruns her opponent. (right)

freshman

Cindy Probus, at midficld, attempts

to regain control

of the ball for

the Jackets.

(middle right)

forward

Michelle Ruhlman

runs the ball

down the field.

(far right)
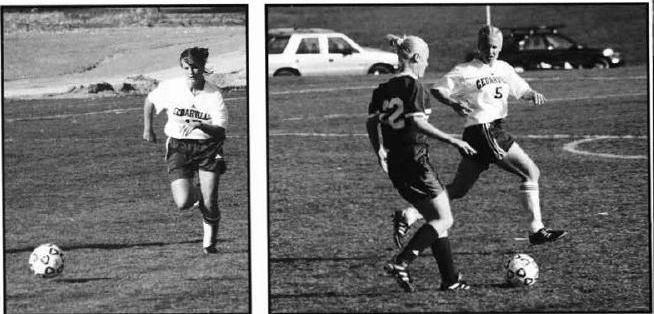

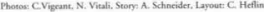
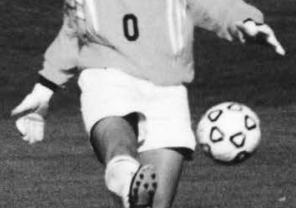

if

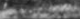
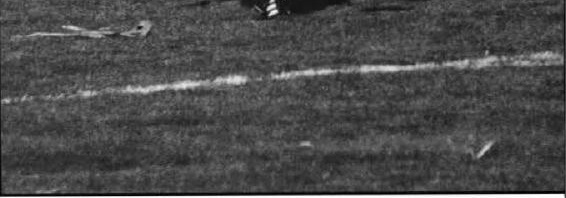



\section{continuing the excellence}

Cedarville's women distance runners added quite a few the team finished in at least the top half of all finishing teams. accomplishments to their lofty history this season. Their Cedarville was chosen to host the anual National Chrissuccess is perhaps even more impressive considering the fact tian College Athletics Association Cross-Country Chamthat the roster included more new members (seven freshmen and one sophomore) than returning members (seven- with only one senior).

In both the Rio Grande and Wilminton Invitationals, the girls took top honors for the day. Not only did they win, but they won in style. In both races, the

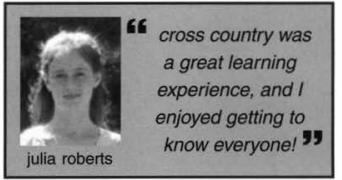
pionship. The race was run at John Bryan State park in Clifton. The team finished third, directly behind perennial powerhouses Malone and Spring Arbor.

Coach Elvin King, now in his 31st year at Cedarville, said that his primary goal this year was simply "to keep the moLady Jackets posted a clean sweep, the first five places mentum going" from past seasons. Given the successful overall.

They proved that not only were they capable of running great races, they were able to do it consistently. In every race, tradition, but also added to it. season and positive outlook for seasons to come, it was safe to say that the Lady Jackets not only continued their
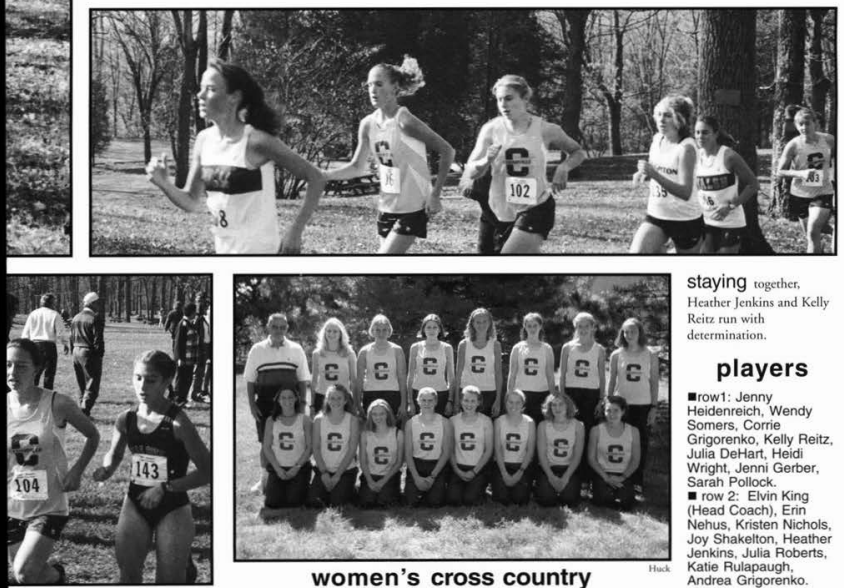

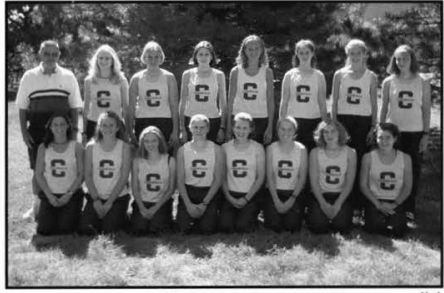

women's cross country staying together,

Heather Jenkins and Kelly Reitz run with determination.

\section{players}

mrow1: Jenny

Heidenreich, Wendy Somers, Corrie

Grigorenko, Kelly Reitz, Julia DeHart, Heidi Wright, Jenni Gerber, Sarah Pollock.

-1 row 2: Elvin King (Head Coach), Erin Nehus, Kristen Nichols, Joy Shakelton, Heather Jenkins, Julia Roberts, Katie Rulapaugh, Andrea Grigorenko. 


\section{striving past the challenges}

The men's cross country team experienced a season full of was the loss of Matt," said King. "That defined the quarter challenges. Although they finished fourth at the National for us. The athletes showed a lot of courage in being able to Christian College Athletic Association meet, they didn't pull the season together." The runners found themselves attain their goal of going to the NALA. "It was a little disap- having to compete while they went through the grieving pointing," said Jody Fox. "We were hoping to qualify for process. "Losing Matt definitely had an effect on the team," nationals, but we did all we could do. We left this year in the said Eddie Nehus. "He was a top runner, and I think the Lord's hands."

Head coach Elvin King emphasized the fact that the team still had a successful record. "We had an excellent season," he said. "We beat $85 \%$ of the teams we ran against. At the end of the year we were not ranked high enough to gotonationals, but the men's team

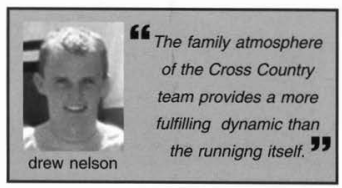
was strong enough to beat the team that was ranked thirteenth.

The team experienced tragedy when teammate Matt Hadler died in a motorcycle accident. "The major factor of the season would be working hard right now and doing his best.

That really encouraged me and gave me something to strive for."

season might have turned out differently if he'd been running."

Fox found his motivation in Hadler's example. "Matt was a really good friend, a really positive influence. $\mathrm{He}$ was doing his best, putting in the extra miles. During the season I told myself that Matt

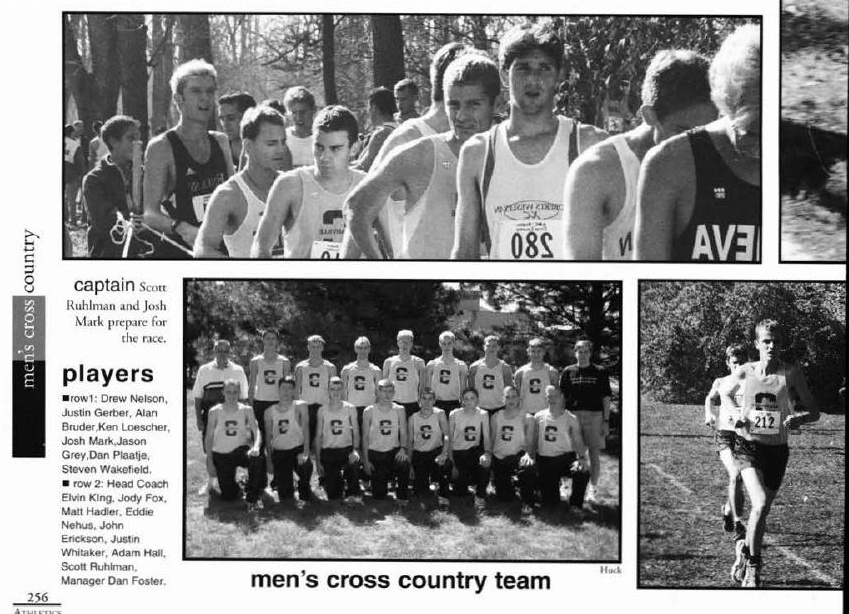




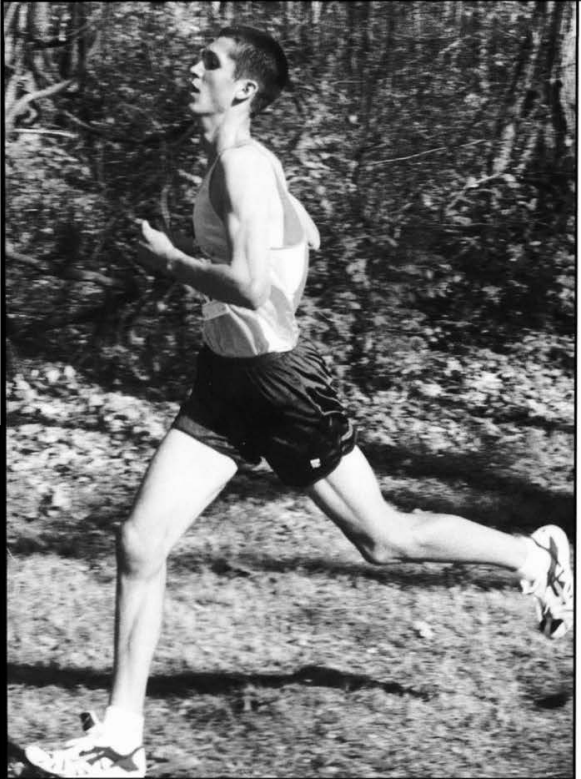

jody Fox strides forward as he races towards the finish line

\section{stats}

- The 1999 men's cross country team finished 4 th in the National Christian College Athletic Association meet, held at John Bryan State Park in Yellow Springs, Ohio.

\section{stars}

- Justin Gerber, sophomore. eamed himself a spot at the NAIA Nationals, in Kenosha, Wisconsin. - Sophomore Jody Fox finished first at the Wilmington Invitational on October 15, at Wilmington College.

\section{scores}

\section{CEDARVILLE:}

- vs. Rio Grande

- vs. Midwest Collegiate

- vs. Friendship |

- vs. All-Ohio

- vs. Wilmington

- vs. Gettysburg

- vs. American Mideast

- vs. NCCAA Nationals

- vs. NAIA Nationals
1 st of 3

6 th of 26

4 th of 14

11 th of 38

1st of 15

3rd of 33

2nd of 8

4 th of 21

(no team)

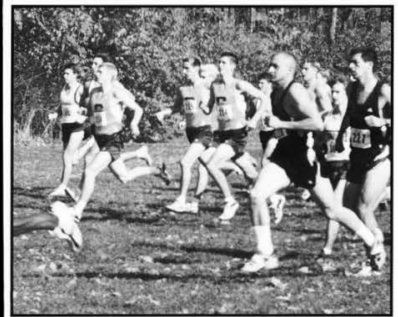

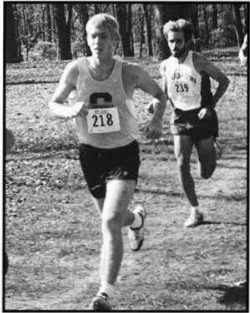

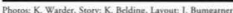

alan Bruder paces

himself mid way through the race. (left)

the Cedarville men

start the AMC

Conference race.

(left middle)

ken Loescher

works to move up in

the race.

(far lefi) 


\section{stats}

- The women's basketball team finished 24-15 overall.

- The team comleted the season with a $15-9$ in the American Mideast Conference.

\section{leading scorers}

- Julie Nourse 14.5ppg, Amanda

Porter 13.1 ppg. Kristen Rossotti

$11.8 \mathrm{ppg}$

\section{scores}

CEDARVILLE:

- Olivet Nazarene

- Roberts Wesleyan

- Taylor

- Madonna

- Huntington

- Bluffton

- Rio Grande

- Grace

- Concordia

- Walsh

- Seton Hill

- Tiffin

- Mount Vernon Nazarene

- Notre Dame OH

- Point Park

- Geneva

- Wilmington

- Shawnee State

- Rio Grande

- Saint Vincent

- Wilberforce

- Malone

- Point Park

- Geneva

- Urbana

- Ohio Dominican

- Wilberforce

- Saint Vincent

- Central State

- Seton Hill

- Geneva

- Spring Arbor

- Saint Vincent

$65-70$

84-74

75-71

80-66

64-66

82.80

65.97

69-59

70-76

71-63

98-88

$67-63$

$64-54$

64- 49

78-60

76-55

54-73

63-105

$71-75$

68.85

84.54

69-75

75-61

65-73

75-79

63-76

89.62

74.87

60-76

82-64

79-76

62-73

74-75

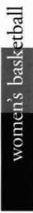

\section{amanda}

Porter goes all out

at practice. (right)

charity

Cole guards with great intensity. (middle right)

pattie schryer aims up for the shot. (far right)
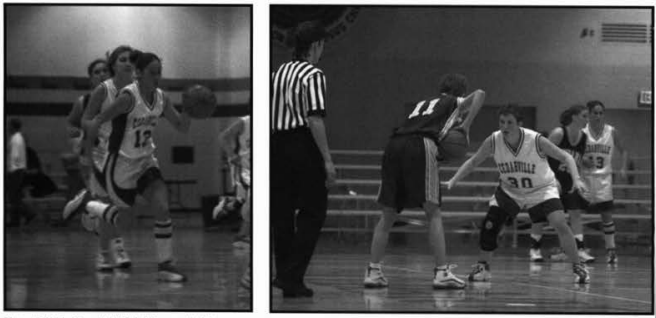

Phetos: S. Hudks Story: R. Mithobell, Layour. E Kelly 


\section{committed to the game}

The 1999-2000 Men's Basketball team knew they were going to face a rough season. They had lost several key players from the previous season.

The men faced a very tough schedule in what turned out to be the last season for head coach Jeff Reep. In the latter part of the season, Reep announced that he would be resigning at the end of the season. As a team, they set feasible goals that they would strive to reach throughout the season. They wanted to strive to be the best that they could be as a team and as individuals in

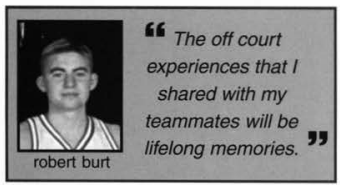
various aspects, which included athletics, academics, the triumph of victory. and spirituality.

The coaching staff felt that there was a genuine season. They played with a lot of heart and never gave up.

effort to be the best that they could be as a team, but each individual would have to examine himself to see if he met the individual goals. Another goal that the team set was to see improvement in the team through daily practices and as the season progressed. Coach Reep felt that the basketball team achieved this goal.

Through hard work and team play, they did improve. Although the men lost several games, the team never let the opponents go without a fight, and when they won, they relished in The guys never quit this

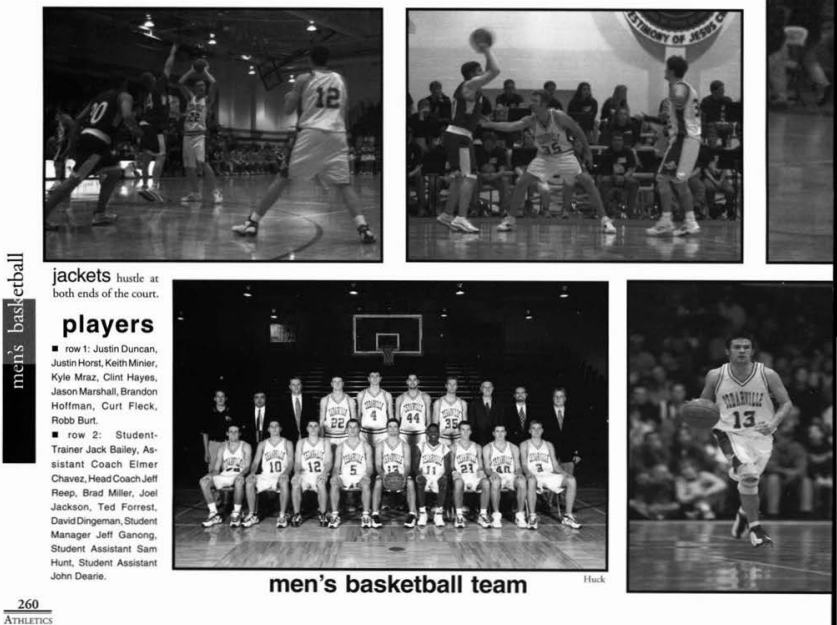


the bee, along with a group of students, "back the jackets" by screaming for their classmates as they compete in basketball.

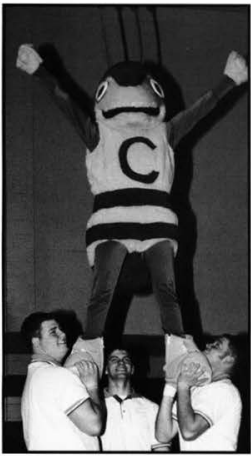

jared Koverman, Jocl Adams, and Josh Knorr lift up the Cedarville mascot to fire up the crowd.

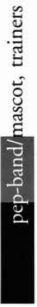

members of the pep band provide music to encourage the fans and to show their support to the athleic teams. (below)

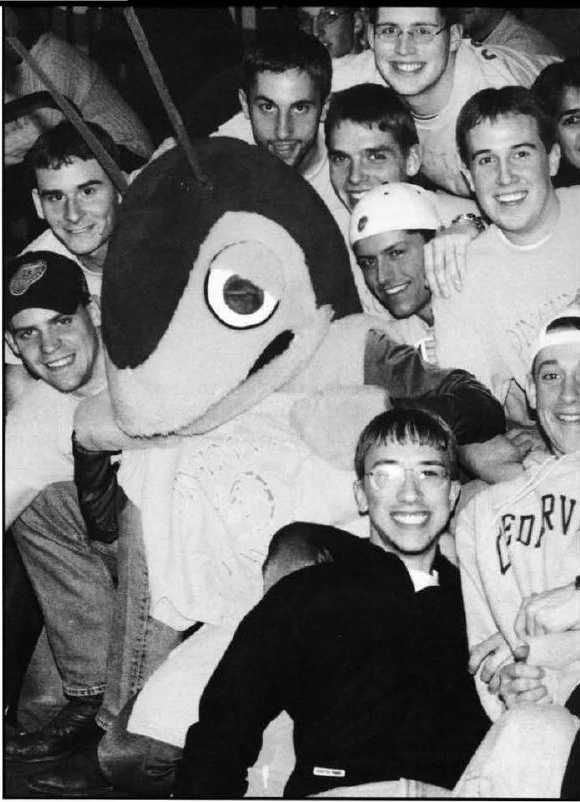

junior Amy

Nyhuis and trainer

Mclissa Turnick

prepare for practice with the Cedarville

women's soceer

(middle right)

expressing

his school spirit is

Freshman, Josh

Haynes.

(far right)
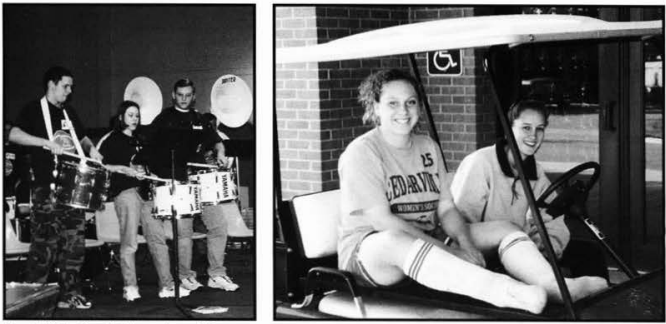


\section{promoting school spirit}

The Cedarville Cheerleading squad had another friendships during the many hours spent together. Often successful year of adding excitement to the home and they would get together at Coach Scott's house for pizza and away basketball games. The team consisted of seven fellowship. Freshman Bill Kane said, "Not only were we guys and six girls and was led by Coach Dawn Scott, part of a team, but we were close friends."

with captains Kristin Hoovler and Andrew Howe. Later in the year Josh Sherwood replaced Howe as captain.

The team entertained and energized the fans with their many spirited routines and catching enthu.

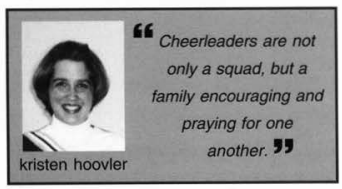
siasm. Dedicated to doing
Once again the team verse and focus of the team's attitude this year was Phillipians $2: 3,4$ which says, "Do nothing out of selfish ambition or vain conceit, but in humility consider others better than yourself. Each of you should not only look to your own interests, but also to the intheir best and making difficult routines seem easy, the terest of others." The team took this verse to heart and squad practiced two times a week for about three hours. applied it to their lives as they encouraged one another The team grew close together and developed close and got along very well.
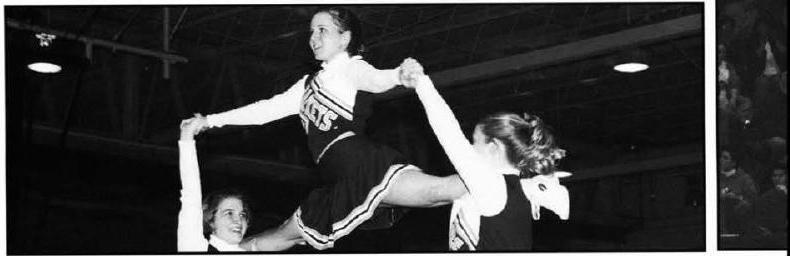

syndi wilholm performs a stunt for the crowd during half-time.

\section{members}

- row1:Assistant Coach Sara Hennis. Syndi Wiholm, Kristin Hoovler, Michelle Drumheller, Courtney Blake, Jackie Ayetle, Nicole Sinzinger, Head Coach Dawn Scott

- row 2: Jeremy Halliker, Josh Knorr. Andrew Howe, Joel Adams, Bill Kane, Jared Koverman, Eric Phillips
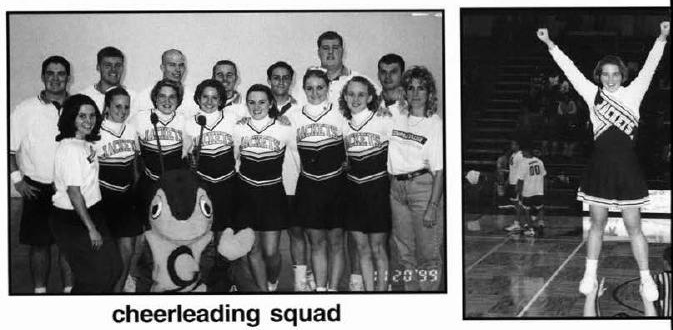
though not usually paired together, Ginger Burler and Elizabeth

Wolfe make a good doubles team.

\section{stats}

- The women's tennis team finished with a $12-6$ over all record.

- The team finished with a 5-1 record in the AMC.

\section{stars}

- Senior \#1 seed Casey Ruffin finished her career with a 37-21 record.

- Freshman \#6 seed, Jennifer Roman brought in the most wins with 12.

\section{scores}

\section{CEDARVILLE:}

- Carloton (L)

- Mount St. Joseph (W)

- Shawnee State (W)

- Ohio Northern (W)

- Tiffin (W)

- Dayton (L)

- Walsh (L)

- Malone (W)

- Muskingum (W)

- Geneva (W)

- Ohio Wesleyan (L)

- Wittenberg (L)

- Transyivania (W)

- Seton Hill (W)

- Findlay (W)

- Malone (W)

- Walsh (W)

- Palm Beach Atlantic (L)

\section{senior}

Julite Gregory

returns a

baseline shot

(right).

focused

on the match,

Cascy Ruffin

prepares to serve (middle right).

\section{sophomore}

Arny Gregory.

winds up for a

power back hand

(far right).
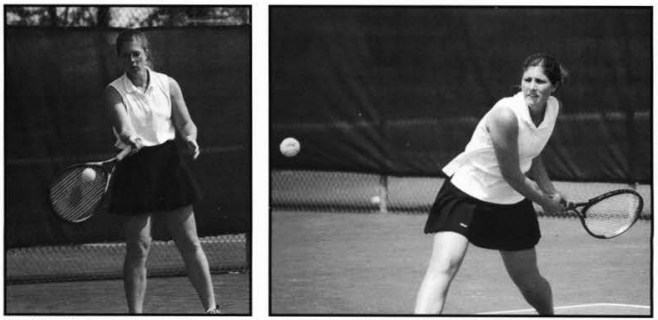


\section{match point}

The Women's tennis team had an exciting line up of mostly returning players who had depth and experience. Head Coach Dr. Pamela Johnson was very excited about the exceptional team members this year. Five of the players were playing their final year at Cedarville and four already had three years of varsity experience. The number one player from last year, Casey Ruffin, was back and continued to lead the tearn. Not only did the team have experienced players but outstanding freshmen also. Freshman Amy Reed was the number three player, and Freshman Jennifer Roman made sixth place. "I've really enjoyed the experience of playing college

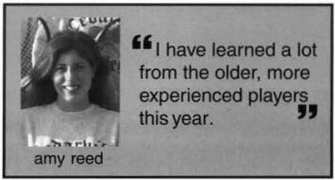
tennis on a strong, competitive team, and as a freshman 1 have learned a lot from the older, more experienced players this year," said Reed. The season started off with a 5.4 defeat at Carleton, though Ruffin did begin her senior season with a victory and $\# 1$ singles. Cedarville

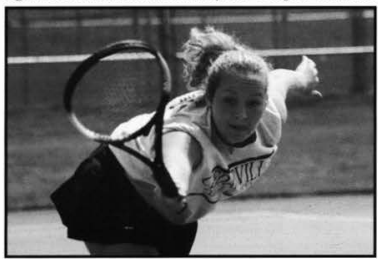

way or another. They are an incredible group of girls who work hard and have a great time doing it!" It was an exceptional team and an exciting year.

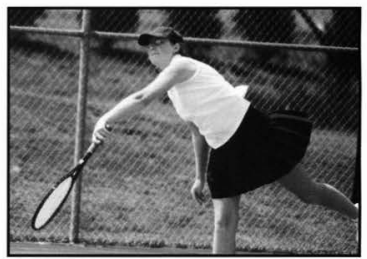

Huck

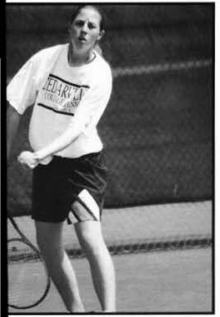

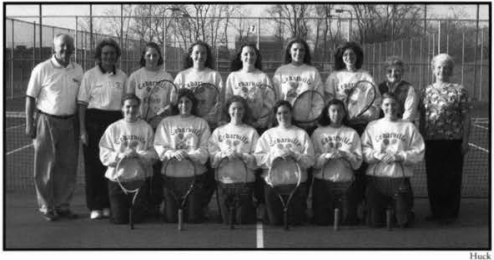

women's tennis team the women's team does well on both sides of the net.

\section{players}

- row 1: Jennifer Roman, Amy Gregory, Elizabeth Wolfe, Amy Reed,

Angela Mast.

Ginger Butler.

- row 2: Assis-

tant Coach Cliff Johnson, Head

Coach Pam

Johnson, Casey Ruffin, Elissa Dvorak, Alison Warren, Angela Seeley, Julie Gregory Ast. Coach Dee Morris. Chaplain Eleanor Taylor. 


\section{love doesn't win games}

The men's tennis team had another successful year. The team consisted of five of the top players from last year, a sophomore who remained undefeated his freshman year, and five outstanding freshmen. Head coach and Cedarville alumni, Aland Edlund, led the team for his sixth season. He was highly respected and an outstanding coach, according to Senior James Metsger, who said, " Coach brings to the team experience from a program with a winning tradition. Hisprioritiesare God first, then school, and then tennis. His proper perspective on life allows for the team to enjoy tennis as a great opportunity while in college, but not the end all in life."

The season started off well with a victory over Fairleigh $6-0$ in their first match. The number one player on the team, Senior Eric McVey, played in this match despite just overcoming the flu. They lost the second game at Carleton, by $5-2$. They opened their first home game with a $9-0$ victory over Bluffton. Cedarville lost only 8 games total in the match.

Another high point of the season was the 9-0

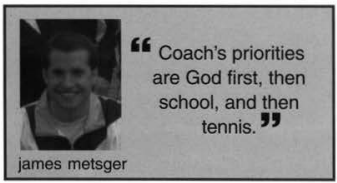
conference win over St. Mount Joseph. Cedarville swept all nine matches in straight sets. Then they opened the American Mideast Conference game against St. Vincent with another 9-0victory, losingonly 7 games this time in the entire match. The team finished the year with an impressive 14.5 record over all and a 3-1 record in the American Mideast Conference.

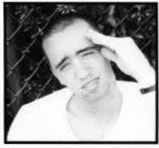

freshman Victor Puhy takes a break from the hot sun.

while at a tournament. the team strikes a creative

pose,
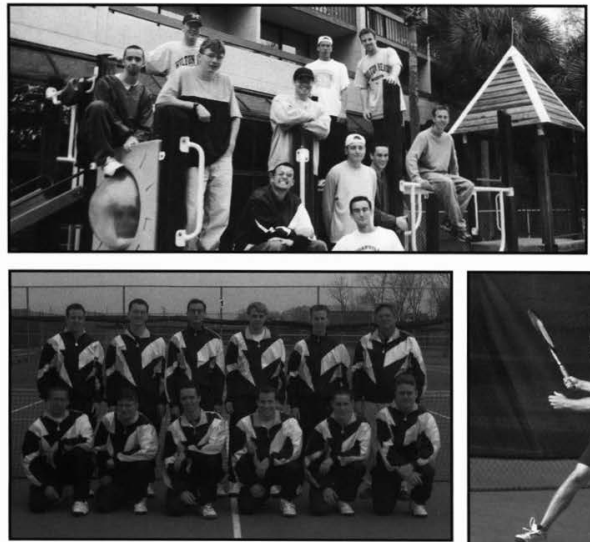

men's tennis team

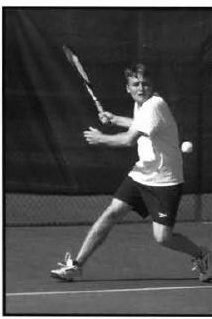

players

- row 1 :

Lance Hart.

Pete

Springirth,

Victor Puhy,

James

Metsger, Dan

DeHaan,

Aaron Franke.

- row 2:

Jason Hall,

Ben Harreld,

Jeff Powell,

John Bailey,

Eric McVey,

Alan Edlund. 
with intensity,

Matt Dunn

watches his tee

shot.

\section{stats}

- The men's golf team took first place in the Southwest Ohio Challenge.

\section{stars}

- Matt Dunn maintained a season average of 78.2

\section{scores}

\section{CEDARVILLE:}

- Muskingum Inv.

- Shawnee State Inv.

- Urbana Inv.

- Tiffin Inv.

- Southwest Ohio Challenge

- Walsh Inv.

- Mount Vernon Inv.

- Cedarville Inv.

- American Mideast Conf.
11 th of 13 5 th of 8

6 th of 11

5 th of 7

1 st of 3

7th of 9

8 th of 9

4 th of 5

6 th of 7 proper form

is essential to

any golf shot

(right).

ben Borich

studies the green

as he prepares

for his next putt

(middle right),

pete Vischer attempts to strike a shot to the green

(far right).
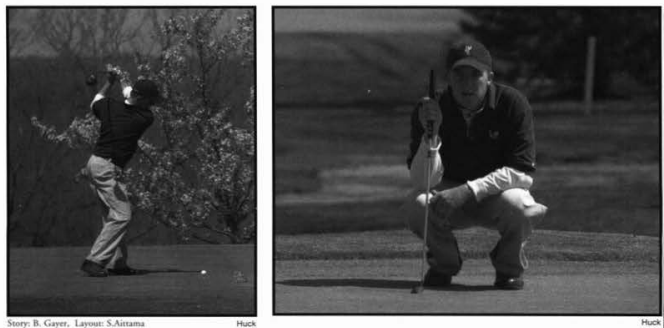


\section{shooting fore the green}

During the spring, the Cedarville Golf team had to Playing in the number two spot was freshman Craig play against the odds to start building a successful Bennington who averaged 82.8 strokes per game. program that will be competitive in the AMC. Youth Coach Jim Kragel finished up his sixth season as was a factor as the team was comprised of two juniors, coach and was optimistic about the bright future of two sophomores, and three freshmen. There were ups and downs in match scores partially due to inexperience.

"If we could get all five players to play well at the same time, we could do some dam. age in the big tournaments,"

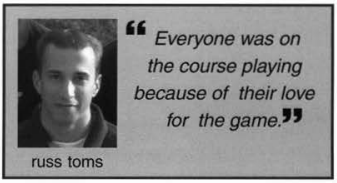
said junior Matt Dunn. the team. "I really believe this is the strongest group of golfers we've had since I've been here," he said.

"We have made a commitment as a team to really try and be ready fo the upcoming season. Al. though we are still a very young team, we now have a group of golfers that on any give day could shoot a very good score for us," Dunn led the team at first seed with a 78.2 season any give day coul
verage, which was the best of his college career. Kragel added.
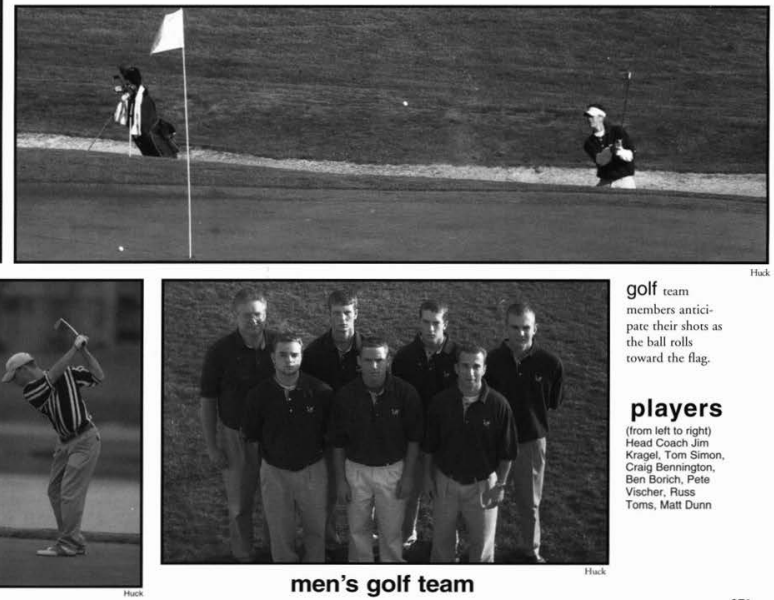

golf team

members antici-

pate their shots as

the ball rolls

toward the flag.

\section{players}

(from lett to right)

Hoad Coach Jim

Kragel, Tom Simon,

Craig Bennington,

Ben Borich, Pete

Vischer, Russ

Toms, Matt Dunn 


\section{field of dreams}

Underthenew leadership ofCoach Sue Carpenter, Cedarville really showed when they went on to win their next four softball had a turn-around season last year. Looking back over games over Saint Vincent $(4-0,4-1)$ and Seton Hill (10-0, the previous two years with $4-21$ and $9-29$ records, the 2000 14-6). After losing twice to Ohio Dominican $(0-2,0-9)$, the season was definitely an improvement with an overall record of Lady Jackets split with both Mount Vernon Nazarene (2-1, 24-15 and a $15-9$ record in the AMC.

Beckey Summers, the catcher and graduating senior, said, "A lot of the turn-around occurred because of the many first year players on this team. The freshmen we have on the team are excellent."

Another addition to the team

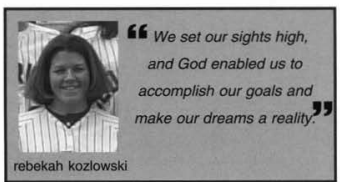

1-7) and Shawnee State (6-2,3. 11) where they hit their season high of 15 hits in a game.

The following two weeks were remarkably good (and busy) ones for the Jackets as they won eleven out of fourteen games.

After Oakland City

eliminated Cedarville from the NCCAA nationals, Cedarville was pitcher Megan Peterson, who led the team to 20 victories, the most any pitcher had ever had came back to win the last four games of the regular season. at Cedarville. Although the opening games were disappoint- Tocap off 2000 season, the Lady Jackts then went on to take ing losses to Ohio Wesleyan, the team's hard work in practice 3rd place at the NAIA Tournament.

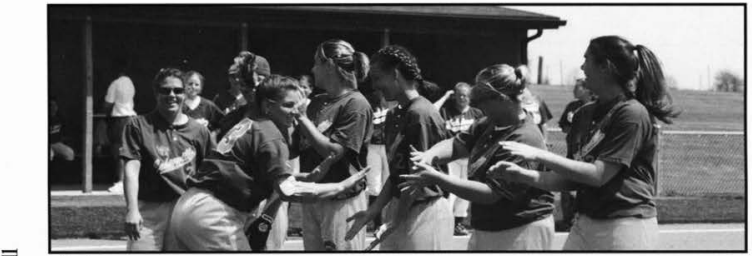

lady Jackets bave spirit

\section{players} - row 1: Kim Ediund Sue Carpenter. Denaye Hilty, Sarah Bishop,Becky Kozlowski, Elissa Morrison, Beth Weaver, Abby Stafford Becky Summers, Annie Millor.

- row 2: Siobhan Fagan, Jill Butz, Julie Burt, Natasha Boyce. Sarah Tsermengas, Megan Petersen. Debbie Krick, Michal Witt, Stephanie Gillett. Kevin Carpenter

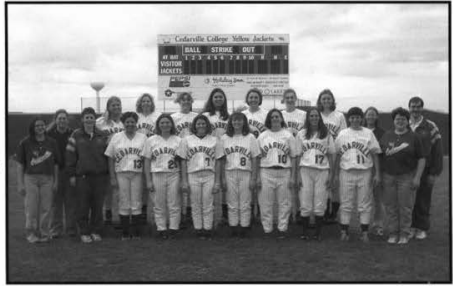

women's softball team

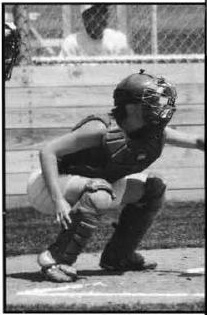


"having fun and coming together as a bunch of guys with a common goal is an incredible experience," says Jacket'scatcher Bryan Mangin.

\section{stars}

- Mican Hutchins, Bryan Mangin, Matt Schroder, and Scott Van der Aa made the NCCAA Regional First Team.

- Micah Huthcins and Bryan Mangin were named Honorable Mention in the AMC.

\section{scores}

CEDARVILLE:

- Cumberland

- Wittenberg

- Carroll

- Webber

- Husson

- Warner Southern

- Hiram

- Malone

- Oberlin

- Capital

- Walsh

- Thomas More

- Shawnee State

- Mt. Vernon Nazarene

- Saint Vincent

- Tiffin

- Mount St. Joseph

- Ohio Dominican

- Urbana

- Shawnee State

- Geneva

- Rio Grande

- Wittenberg

- Point Park

- Asbury

- Indiana Wesleyan

- Spring Arbor

- Indiana Wesleyan

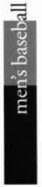

"coach hughes taught me much more about the game, especially to pay attention to the small details" says Chad Hofstetter. (right)

the team meets at the mound to receive instruction from Coach Hughes. (middle right)

"it is sweet for me to think of the potential that this team holds for the next few years," notes senior Nate Verwys. (far right)
13-7

2-6,10-14

4.13

4-1, 2-14

5-9, 0-4

2-8, 1-7

$7-0,8-6$

16-21

1-5, $1-11$

2-6

7-11

1-9, 4-10

4-5, 8-13

4-5, 3-5

0-3

$2-21,2-7$

$7-9,2-7$

5-10, 6-9

3-7, 2-13

11-10

0-11, 3-10

9-2, 2-10

14-13

1-4

3-7
3-4, 5-10

0-10

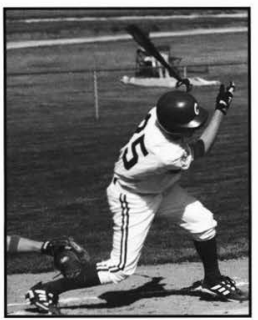

Photas CVigeant, Stary L Huphes Lrout: C. Heflis

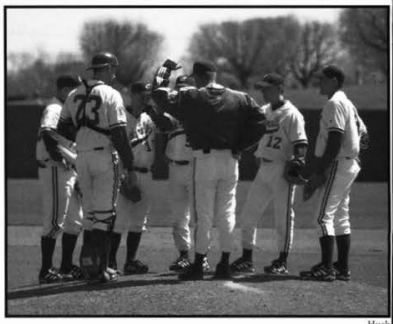




\section{jackets strike back}

The men's baseball team showed a great deal of out of the team's 9 wins on the mound.

improvement from the ' 99 season when they won only 2 games. This year the team went 9 and 36 .

Highlights to the Jackets season included quality wins such as a $4-1$ victory over Warner Southern in Florida(ranked in the top 15 all year), a series sweep of local rival Wittenberg, and an upset of Indiana Wesleyan(30 wins on season) in the NCCAA Regional in Spring Arbor, MI.

The men's baseball team made improvements in almost every statistical category. The team raised its batting average by 40 points, and the team's pitchers lowered their ERA by 2.82 runs/game. Matt Schroeder led the ream in hitting with a .326 avg. Micah Hutchins and Bryan Mangin hit .325 and .323 respectively. Scott Van Der Aa earned 6

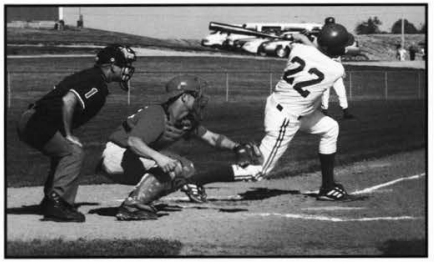

baseball team
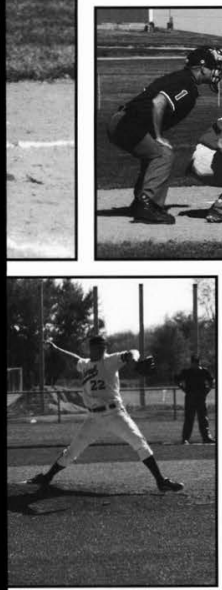

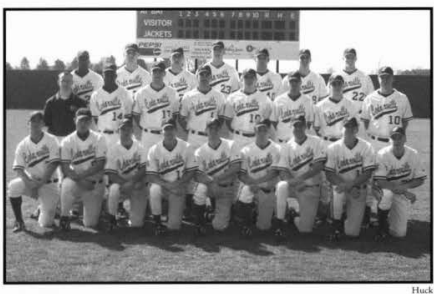

nate verwys makes a mark as a hitter and pitcher in his senior year as co-acptain for the yellow jackets.
"We definitely showed improvement this year," said coach Hughes."And I feel like we were successful in laying the groundwork for a positive future. We have a long way to go to get where we want to be as a program but I think we can be somewhat pleased with

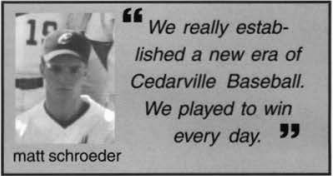
the progress made this season. Nate Verwys was our only senior, and our youth showed often. We learned a lot about baseball this year, and if we continue to work hard and be willing to learn, brighter days are ahead." junior co-captain

Tim Sasticwaits

patiently for his pitch during a home game.

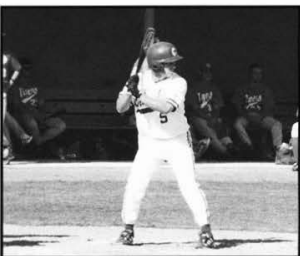

players

- (Kneeling $\mid-r$ ) Aaron Craft, Dan Rickett, Tim

Sastic, Aaron Statler, Chad Ireland, Matt Schroeder, Trevor Creeden, Dana Gerber. Chad Hofstetter.

- (Middle 1-r) StudentTrainer Chuck Archibald. Samuel Hutchins, Kyle Gerber, Drew Bennett, Ben Saturley, Nathan Coomes, Craig Sullivan, Head Coach Greg Hughes. - (Back l-r) Micah Hutchins, John Myers, Josh Logan, Bryan Mangin, Scott VanDerAa, John Bourdeaux, Nathan Verwys. 


\section{success in small numbers}

The women's track team lost a number of seniors last year, and consequently the team was a little smaller. Not allowing their deficient numbers to hold them down, the women performed well throughout the entire season. As a team, they placed 2 nd in the AMC meet and 2 nd in the NOCAA championship meet.

Kate Beatty had a great year competing in the AMChigh jump and 100-meter hurdles. She also was All-American at the NAIA outdoor championships in the high jump with a 3 rd place finish.

Kelly Reitz had a good year in the distance events and capped it off with a 6 th place (all-Ameri-

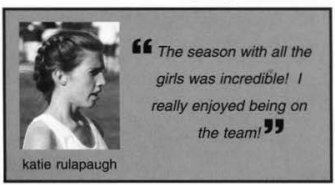
can) finish in the Marathon at the NAIA outdoor championships. Erin Nehus was the team's top performer in the distance races on the track and was a NAIA championship qualifier, as well as AMC champion in the 1,500-meter run.

Sandy Swales set a school record in the Hammer throw and was NCCAA champion in that event, as well as a qualifier for the NAIA meet.

Jodi Quint set a school record in the Javelin throw, was AMC meet champion, and qualified for the NAIA outdoor championship. Jen Heidenreich had a very good year in the 400-meter hurdles and the triple jump. She won the triple jump in the NCCAA and $\mathrm{AMC}$ championship meets, and qualified for the NAIA outdoor championships.

The women's track team performed well throughout the season. They built a winning season out of a young team, and they hoped to accomplish even more in the future.

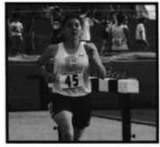

sarah Polluck runs hard in a home meet.

the whole team recieves an award for a great year's work

\section{players}

- row 1: Jeniter

Heidenreich, Julie DeHart, Andrea Grigorenko, Jenniter Walker, Jennifer Gerber, Heidi Wright, Corrie Grigorenko, Rebekah Millot.

- row 2: Erin Nehus, Sarah Pollock, Callie Edgington, Kelly Reitz, Beth Waiters, Jessica

Alexander, Hannah Cook,

Danielle Davidson, Wendy

Somers.

- now 3: Head Coach

Paul Orchard, Manager Jeneel Forward, Sharon Wright, Kate Aulapeugh. Kate Beatty. Shannon Fries.
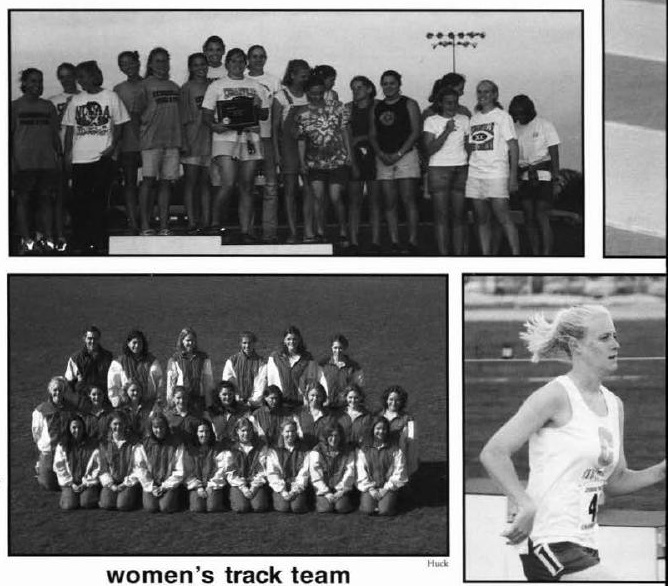
josh Marks

contends with a

steeple chase

hurdle and water

\section{stats}

- The team finished 2 nd at the AMC meet.

- The team finished 5 th at the NCCAA Nationals.

\section{stars}

- Shawn Graves broke the AMC record in the hammer throw.

- Cliff Reynolds broke the AMC record in the 400-meter hurdles.

\section{scores}

- Ohio Norther Indoor

- Cincinnati Open

- Findlay Indoor Im.

- Ohio Northern Mid

- Denison Indoor Inv.

- All-Ohio Indoor

- Ohio Northern Indoor Inv

- NAIA Indoor Nationals

- Florida State Relays

- Cedarville College Open

- Bowling Green Classic

E Miami Inv.

- American Mideast Cont.

- Indy Relays.

- Cincinnati inv

- NCCAA Nationals

- NAIA Outdoor Nationals

3rd of 5

No Score

No Score

No Score

No Score

No Score

4 th of 10

31 st of 38

No Score

No Score

No Score

No Score

2nd of 6

No Score

No Score

5th of 15

23rd of 67
Cliff Reynolds

recieves an

award with

Coach Orchard

(right).

with

intensity, Drew

Nelson moves up in the pack. (middle right)

jody Fox stretches ou his stride. (far right)
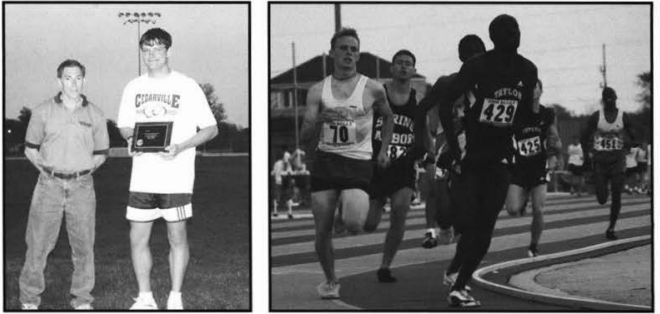

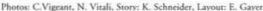

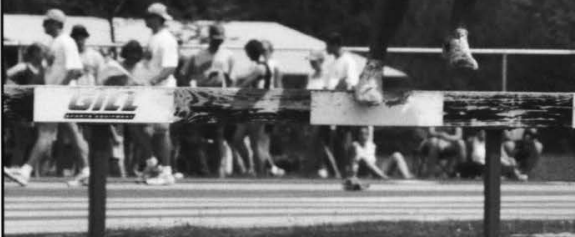

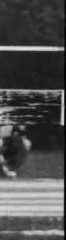
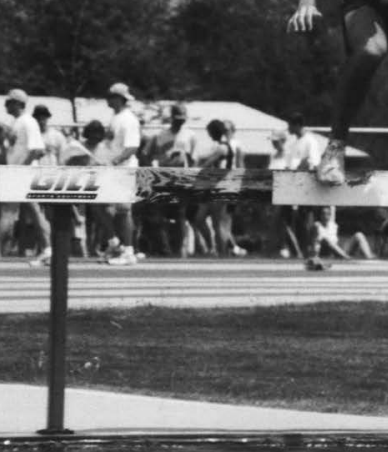


\section{running-throwing-jumping}

The men's track team experienced a successful season despite a rocky start in which they lost three of their best runners. "It got a little tough toward the beginning," said co-captain Eddie Nehus. "It seemed like one thing after the next was hurting us." The season improved from then on, however, and junior Cliff Reynolds was able to conclude, "Some things happened that made it hard and challenging for us. But it was a good season for me, and overall for the team too."

Team members credited part of their success to strong leadership. "Pete [Bednarek] did a really good job of providing direction and unity," said Nehus. Freshman

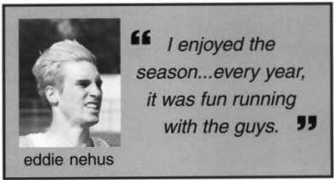
Drew Nelson agreed. "We met at his house a couple of times, and Pete gave devotionals," he said. "I thought it was interesting because he drew a spiritual analogy to track that team members like a shot putter and a sprinter are

mates can help you. But in track, it's just you," said senior Shawn Graves. Drew Nelson added, "Personally, 1 think it's one of the purest of sports."
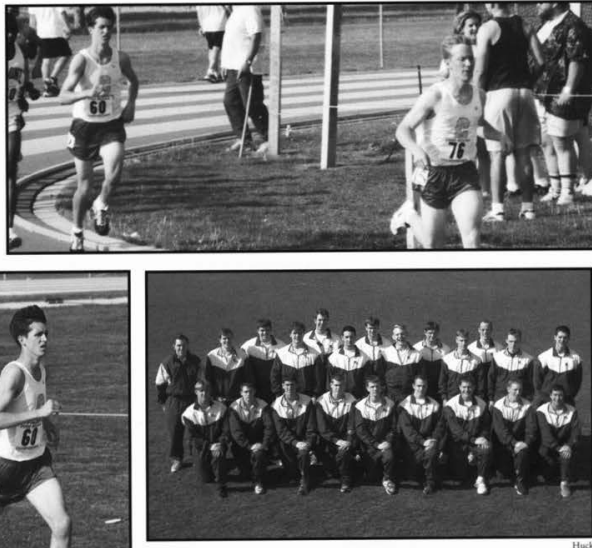

chester Wakefield leads the pack in a distance race

\section{players}

- (Kneeling I-r) Wesley Stephens, Jonathan Clark, Justin Gerber, Ken Loescher Alan Bruder, Nate Jenkins, Adam Hall, Steve Wakefield, Andy Hayes. (Standing I-r) Head Coach Paul Orchard, Manager Dan Foster, Carl Traub. Shawn Graves, David Dingeman, Brittain Bollenbacher, Andy Paugh. Eric Yearley, CEiff Reynolds Eddie Nehus, Jacob Abbs. Drew Nelson, Jody Fox. 
bill Stewart

makes contact

during an

intramural

softball game.

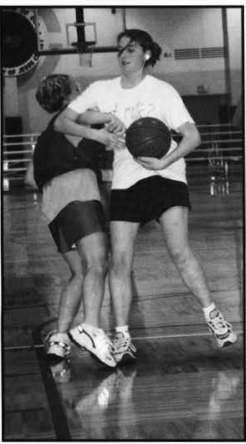

girls' intramural basketball can become a full contact sport.
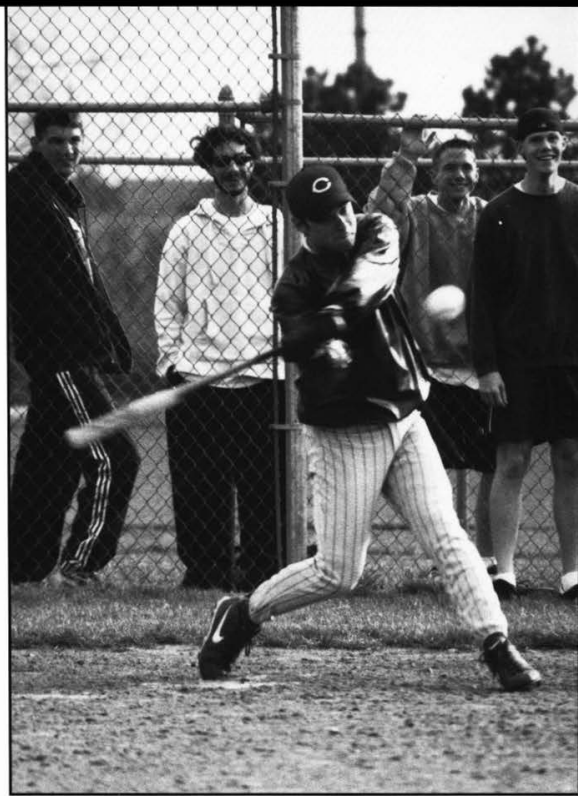

क्
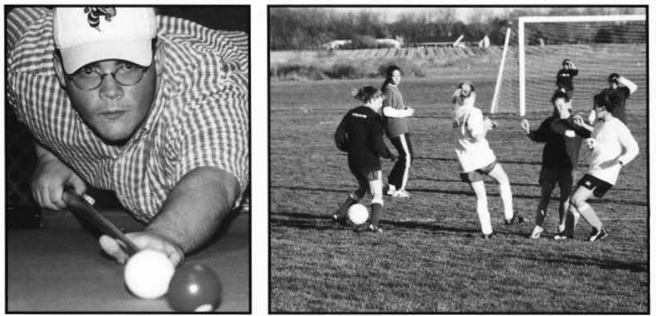

ryan Elam, Joshus

Williams, and Dan

Miner serve as

cheerleaders when not

in the game.

(far right) 


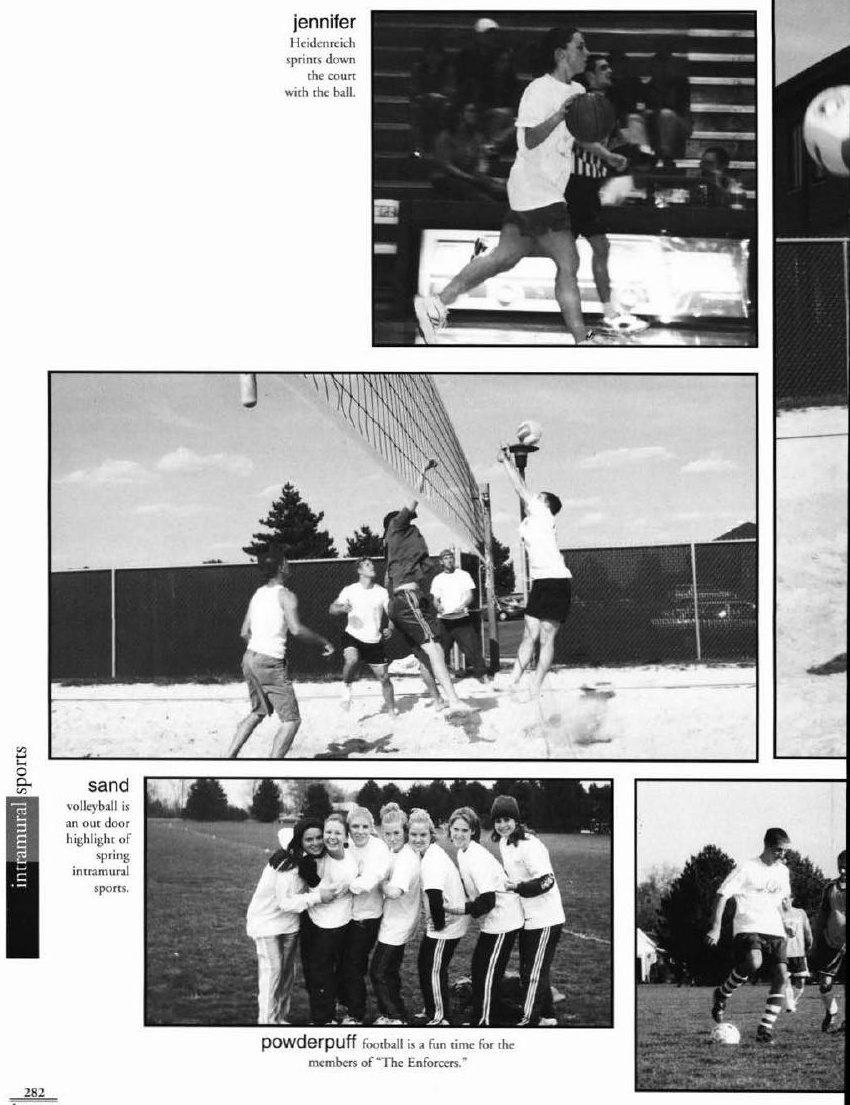




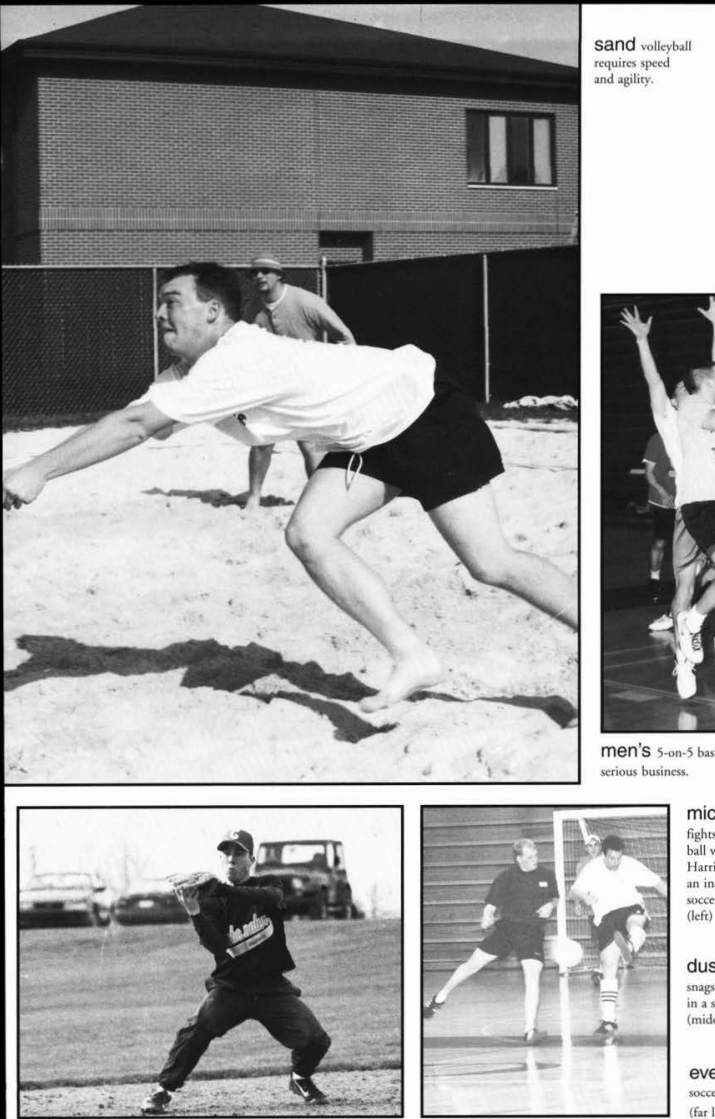

Photos: R. Bedker, Layour: 1.Bumigaraed michael Bochm fights for the ball with David Harrison in an indoor soccer game. (left)

dustin Crider snags a nasty hop in a softball game. (middle left)

\section{even intramural} soccer is slow at times. (far left) 

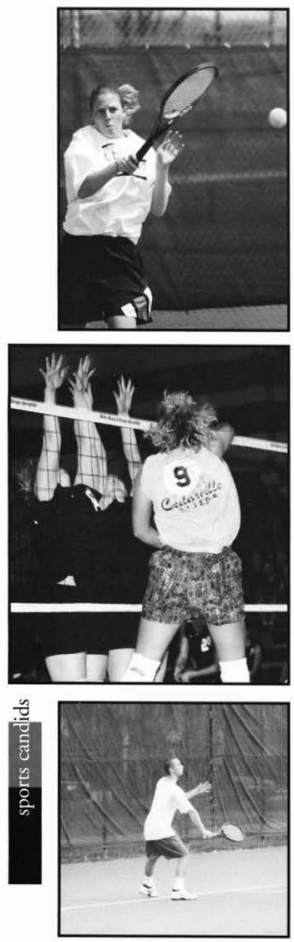
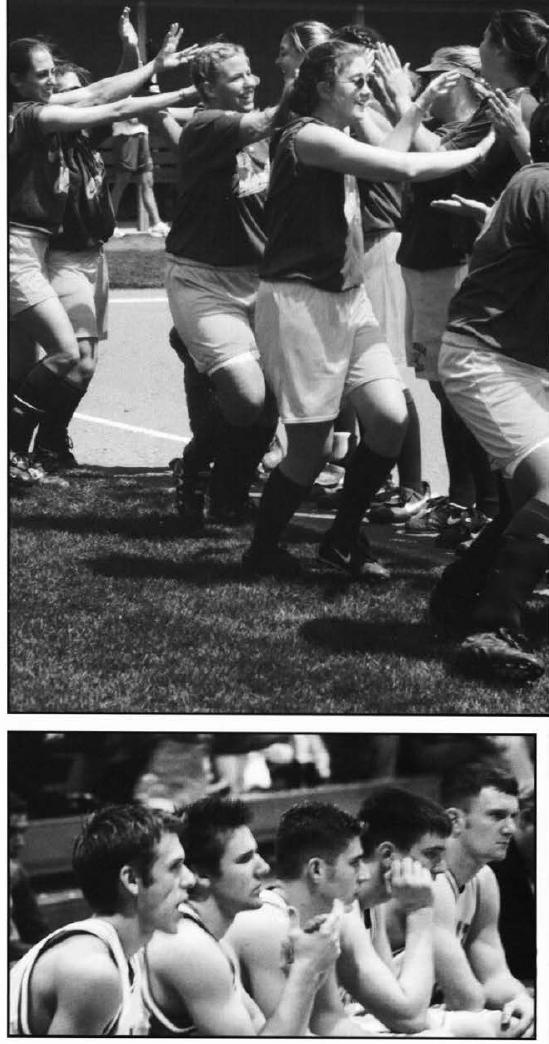
Avestell, Brien 295 Abber. Roberi 71 Abbry, Jennifer 82 Abboes. Tim 132 201 Abbo, Chrivy 192,195 $230,233,233$

Abbus lacol

Abr. Einalarih 82. 213 Ahels. Darid 158.239 Abriham, Pal 145,194 Acket. Matk ISs

Acketman Jesica

Ackrtman Michrlie tis 197,23,

Ater. Culat 82.130

Adam, Melise 8 :

Adama, Dan 252

Adens, Joed 158, 224, 262,

26.4. 265

Adams Tornc 165

Adeolevina, Kolan

Agro, Ruth

tephanic 52.82 .

122218

Asyi. Bamidele 19:

Alierberg. Arik k2, 2 ,

Abasese, Emily 149

Whert Joel 145. 195

Alhettion. Heather 15.

Alecanders Jewica 145, 201.

251. 276

Allcorn, Enic \$:

Allen Resida 159

Allen Derida 149

Allon, Hasnah 82, 24

Alsen, Kara 296

Ifict. Nicole 158

Ullen, Futrous 192. 255,

Allen, Sata 149, 198

Alem. Satah 152, I9]

Allm, Scent 158

Allos. Stuwn 13:

Alen, Titus 82

Nilo. lewd 132.21

Alloy. Leat 82

mamer 158, 100

Nilpon. Brive 145

Alpont Charis

Anan Chules 145

Amtunital Nante 158

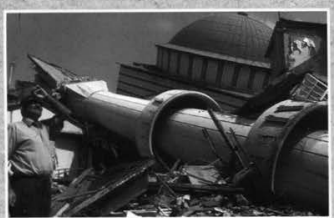

Falf a million people in Adapazari located southeast of Istanbul. Turkey, were left homles by the August 17, 1999 carthquake.

Here a resident looks at the damags, with the collapsed Totiu

Mosque visible in the background.

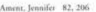

Anoen Jouk 72

Sensuk, Jis 215

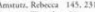

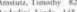

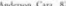

Andersan, Censicis 69

Arderaa, Deäres 158

Anderan, Erin 14

Andersais Lesi 72

Andenas, Lyle 60

Anderaan, Shiekh ifs

Andras. Jill 143 ,

Andras Mtictal 105,224

Anderese Eatily ias

Andivws, Francen

Anejund Dana Ha, 195, 220

Antes Briss 145:27

Antheng, Dewe 253

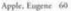

Apple, frenifer 165, 194.

190. 19

Aphrais fard 192. 200.

239

Anchitwald: Onarlas s:

Anchinulit Chux 275

Ar

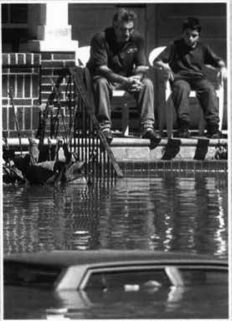

Residents of Lodi, NJ, sit on their front porch surrounded by water and a submerged car in their front yard on September 17, 1999, Lodi was one of the towns it the state that was inundated by flood waters from Hiurricane Floyd

Awery Juth 72

Bader, Mishal 72

Bad, Cariua 83,

Bennington, Craig 27
Biladeas, Kristas 132.212,

Binchfidld. Rebect 159

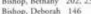

Dathop, John 132

Batheg, Suns 139.272

Birmer. Dowe 146 .

219

Bjerneat Kanen ke

Black. Alius 146

Hisck Dovit 150

Hlock Sient at

Plackbuen, Aveci 84, 280

233

Badow, Abb 146

Blike. Conuay 157

Blake, Countocy 269

Eito les ise 23.

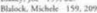

titus a

Bictux Meagan 192. 17

ig?

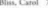

Blodige, Andy 230

Blodpet, Andy 8 .

Blodgati, Jehn fo

Bliodpati, Nets 32 ?

Bloaset. Allivies 199, 194.

20 s

Dbixer, Dan 153, 195, 25i

Dibocen, Mant 159

blow, Tineatiy 146

Blaincenseock. Meks 72

Beckuahiler, Anic 115, 201

$217,218,231$

Beodoen.

Awituly, Niek 218

Ares. Alicia 145, 226

505. Joantuan 16, 201

\section{$\mathcal{B}$}

Bubood, Cheinins 15 Bulans, Enic 158, 215 Buern. Lias 132.200

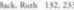

Dader. Ran 72.216

Dacs. Panicile 155

Baliey, Jack 132, 216, 260

Aasiley, John 145, 265. 269

hailey, Medlic s5

Iny. Timothy 145.236

Boicatit, eran. 158

Main, Anerice 155

126 . Wertic as

Salks. Katry 165

Baker, Kríta 150.

Bater trah 118 ,

Baler, trah 136,

Bules Lan 125

Haker, Rance 83

Bake

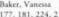

Buker. Wolky s?

Muldwin, Miethew

Balah, Adain

Baland Staver 71

Bund, Geargin

ins, 230

Banka, Benira 132

Funile. Grais its

Bararts lasica
$192,39,282$

tartas Shase

214

Huben. Jack 94

Bush. Blethany 89

Batier, Siany 177

Placker, Malanis 5 ;

Weikow, Tami If5

Burnat Bratfoend

$x+121,132$

Fiarnet, Camirn 65

Barnerie. Amy iss

Barmetie. Amy 198

Barticus lounra 83

Barders, Satah 146

Bars Darid 15\% 13

Bacisa. Kevin 146

Haiss Beth 159

Buminanx, Diveslat

Kuxoer, Detoonh its

Beach, Dree 159,22

Buch, Rached 20, 159,200,

Eattic Amande 15), 201,

206, 279, 239

Barr, Kase 146.27

Beck Deberah is?

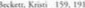

Bectore Binekh 146

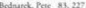

Bedding Amy 140

Eddeng, Kuten A4. 99, 11 .

132

Scill Martiow 11. 146

Bdil. Mrovas 206

Bell. Nathan $15 \%$

Bender, Fris 192, 228, 24

Berinati, Ciscy

Eernett Andien 146, 20S

Beminerr. Dawist

Bernetc. Melissa 159

Mernert sasedy 84, 191

Benoet Siacie 8d 244

138, 242

Beresi Adaim 137

Betmand, Wallian 60

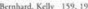

3ery. Derait 65 .

Bcriry, Subrim Sh

Bost, Paul b4

Beats, folf 72, 115,243

BeViea, Nate 146

Bicknell. Mant 24!

Hidek. Robern

Bide. Metivis 29, 2

Bieter Dougitar 159

Bieri, Clent 146

Bimper, Mase 159

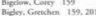

hil, Willy 227,255

Helk Nicole of

Billaby Chad 159

Boditr, Juditis 67

Botraniller, Curies as

Bodeneiller. Todd It

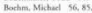

Moskir. Nisole 133

Bositic Mark 16h

Bogsp. Jave 153

thobl, Em
Babap. Natuan 146

Borion, Mrqua 159, 206 Bosma fanice 67

Bexworth 5ay 217

flemau. Hener 152, 23

Dearas. Jekeriy 196.219.

25). 26

Beutiftas, Johe 133, 194

Houverna Rastall 199, 224

Howder Wath

Bowen, Metiwe 159.25

Bowens. Rundall 206

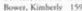

Mene, Pew 133, 270,238

Borevn, fock tice

Bewas, Shethy 160

Bowetrea, Jernes 72

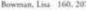

Dorec Kclly ss

Bong. Narahe 1606,272

Bord. Ketly 85, 235

Bond. Matithes of

Bom, Mank 219

Barke, Aundinine 25

Heuts Connir 72

Mndskre, fman 160

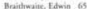

Itrame, Mishat B5, 190,248

Bramener, Miriah! 160

Brambins Call 6 ?

Branoen. Julic 146

Brased. Melanic 20 :

Arzass, Erin 146.210

Brazkavich Star 45

Alernde, Elizalerth 65

Brentianger. Jennifer 23, 195

Bresuen, frindis 60

Brewer Bethany 196

Fauman, Zippotat its

Bect. Abicall 132, 194,205

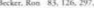

Bithen, Surath 159

Hileck Jin $84,128,226$

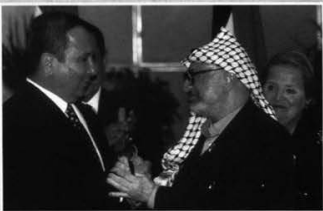

Isracf Prime Minisrer Ehud Barak, Jefi, shakes hand with

Palestiaian leader Yasser Arafat September S, 1999, after signing a land-for-securiry agrecment in the Proptian resort town of Sharm d-Sheikh as U.S. Secretary of State Madeleine Albright looks on. 
Dino: Flond 24,55, 69 Elanaik. Pamely 73
Evelli, Eraily 200,225 Evell. twon 162 ns Endion. Andira 90, 193 204.238

Enertmann lame 60 Fneland Grogry 16 : Eninet, Sandrin 52,62 , Epperthy, Juscin 240 Erickien. John 256 Exdon. Kimbely 148, 227 235, 240, 241, 249

Eaippon. Michat 243

Erepe Sik 212,21

Essen, Anny 16 ?

Ereen Conpl th

Frect Dusiat 68, 127

Eus. Jounas If8

Esio. lod 57, 154. 16 ?

206.240

Bate. Kemp

Exant Enily 148

Evins. Mary 162? 200

Evanu Parncia 148

Eveere. Andrew 162

Everen, Heidi 16

Evem, Heidi 191

Evic, Fin 138, 206,216

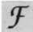

Pasian Curtic 90

Fagan, Suthan 272

Fiska, Kuryi so

Fatloy, Eethany 162

Fatiow, Ander ov

Fundow, Joyce 7

Faflow fula on

Farmer Krain 229

Faimer, Krivin 148

Farrell, Jonuthan 163

Farvil, Aata 162,215

Fausl. Dana 146

Falkncr. Chriay

Falkact, Stan

Fallingo Join 278

Farorite, Hally 20s, 237

Fawce, Jeflery

Fawarn. Cliefond

Fiscet, Jalia 236

Fiwcm, Teary 67

Foldmeien, Matishes 162

Feimlex, Adam 16?

Ferint, Kriatis 162

Feonon Any 39

Femen luchas 169

Fasan. Tim 309

Fegranen, Jennifer 90. 217

Fetpoes, Lence tas 213

Femiander, Furuands 23

$225,231,23$

Furiesa. Michad 238

Feratr. lonh 235, 240

Ferart, Joabur 90

truche Kat 2y $24 \mathrm{~m}$

Findley. Nusile 148, 206.

Finloy, Kari 163

Finnegan, Sech 163,192

Finecy, San To

Finnigan, Julic 90, 220

Fira, Chad 169, 290

Fismin, Mishad

Fishos, Lor 167,200

Ficher. Lera 163.

Fister. Dowd 16

Fisher. Jonh 148

Fisher, Noella to

Fised. Nancy

Firek. Daicy 148. 210.

240. 244

Fare, Mart 90

Fleck. Curric as

Fleik, Cartis 148260

Fienas. Arananda 16, 208

Fleas, Sarah $90,195,2,1$

Flemgr. Denais 64

Fikl. Julic - 227

Flood Jenner 191

Flood Jenniler ity

Fion. Enis tw? 210

Forty. Maris 195, 220

Flamkzr, Ryan 169

Foxtch. Chad 2is

Folliman, Noetlik 79

Follweiler, farik $18 \%$

Folu. Alrue 169

Fulu. Dencida 90

Foote. Mictisa 210

Farni Jaine 169

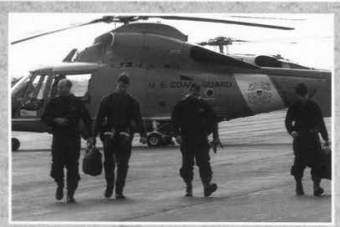

United States Coast Guand jets and four cutters search the Atlantic Ocean off Nantucket, MA following the disappearance of Egypt Air Flight 990 on October 31, 1999 at 2 A.M. The Cairo-

bound Boeing 767 had 214 aboand when it took off from New York.

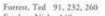

Fonber. Niak tw

Ferterer, Nhthy to

Fonweral, Jendle 225,27

Fover, Din 256,270

Fouser Hyan 192

Foser, Sonya 91 . 104, 202

Fearman. Aares 16x.240

Feurman. Dayid 91

Feus, Jeremy 16

fouwiche, Janie 163

fos. Aebekah of

Fex, Steven

Fakm, Jelie 169. 19

Foneis. Jneph 69

Fuscin, Beinh 64

mank. Draphe 71

Fenk. feteny 91, 195

Frash, Rent 16j

Frenla. Andrea 164296

Funkr. Natus 268

Frandlin, Gary 163

Faries, Ernt 160

Firderick fout 169

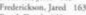

Jeend, Danil to

Fenmin. Crisinpter

Fiene, Karby 67,259

Freers, Michad 149

Frets. Oarinie 66, 248

Frea. Dard 55, 91, 124, 224

Frey, Rachid 169, 194:217

Fichd. Sumenrs 91

Frie, Daniel 30.91

Fricam, Melins 363726

Exchetierg. Sirith i6t 190 .

Fronduti. Jouh 125, 188

Pravduac, lechus 92

Eruminadien 100

Eq Thomen az

Fupo Jonathas 92,233

Fuhe, Darecen 227

Fahi. Dooen in
Falleren. Orritina 92 1s9.

Furmicires 84,9 ?

Fusorin flen 163,275 208

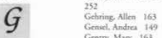

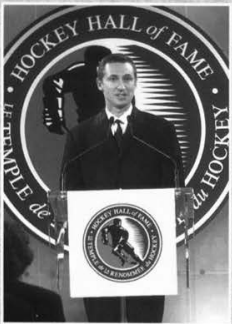

WWLC hockey player Whane Gretzky is inducted into the Hockey Hall of Fame

November 22, 1999. Gretzky bolds the NHI record with 802 goals, 1,851 carcer poinrs. and 2,857 points

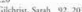

Gollmer, Stevon 65

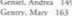

Ceores, Carwt 74

Gerter, Dana 1019, 200

Gerfor. Janifor 112

220, 221

Goodteis. Jarin

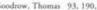

Gatden. Iennifer 136,240

Goigyla, Bath 217,219

Gortwallo Diase 7

Goinnale. Jeniti 95

Gosing Cortal tos

Gran. Thilany $16 \mathrm{a} 192$

Ged, Nioule los

Grahain. Charity 7

Gram, David 63

Gratume, Oharioy 79

Granets, Michael 16:

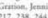

Chums, Shaw 93, 265. 279

iray, Den

Gay, Enily 164.2to

Groy, Kvis 19,186, 201

Greco, Danial 93

Grasa, Melitua 16

Genen. Peter o3

244. 297.299

Githene, Ruy is

Greme, Saril

Gantz. Xyle 45, 92

Garber. Becky

Garnet. Dustin 163

Gind Linday 163

Garecr, Macher 35 ,

Cater, Melinda 92

Geyer, Ben 31, 206, 206,

207,209

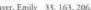

231. 297,299

Givnier $\{y=169$

Ger. Netedith

Gertier. Jualin 226.25\%

Gerber. Kvle 2066.

Gabb. Anna 163

Gebles, Kinten : 27

Catibs tor 62 :

Gideos,

Cridley. Angria 163

Gilber, Nate 149, 200

Gilber, Sarn 215,216

Gillow, Keich 93. 180

Gillespic, Gina 169, 207

Gitlet. Allioge 03, 207

Giliar. Scephusic $19 \%$

207,272

Gilmour, 1 im

Gingrich Antoes

Ginh, foch 235, 24

Giteh, Richard 136

Glawo Kolv 136,61

Gitesem. Kristi 79

Glosiner, Jase 92:99

Godwie Jancph 60

Goxdraita. Sara 93

Gachring. Jessin

Gocer. Slant ing

Coms, stantirs

coctrmasn. Janice 163

Gohaes. Glani 169, 20:

Gombia. Lod 22. 163

Gomins Mansith - 156

Good. Alicia 136. 208 .
Guerete Latie 164

Campreche Orend $16 \%$

Gupritl Aamie 149.177

Cwilr. Past 94, 99, 200, 231

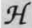

Hatroger, Aaraa 140,243

Hacker, Datid 94,230

Hading. Kelly

Hadiar vitt 10-25

Hecken. Danied 204

Hafter. Fater 136.267

Hagra, Danitile 149.207.

235

Hagrt. Alrohy 136.2063.

Howe Chathes 16- 206

Haier. lehn 164, 225, 25

Hapar. Calit 164, 209

Hain, Kisberly 136, 256

Hahich. Krein 94.128, 198

Hall. Heather 189

Hall, Janes 94

Hall Jamie $123,197,203$

Hall Jaens 146, 264,200

Halleland rachel t64.207

Halleren, Seephen 149

Hallonen, Jemathan ?

Haleve Joeph of

iner, Auran 136

Hatig, Maty 150

une. Megin 150,206

Heater Michad of

Hawerkak, Visoris IG

Hyon, Anar 270

Hyes. Clint 20, 260.261

Hyon Kacit 190.218

Herner, Mark 94

Hanes. Andy 164

Hayng 164,263

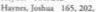

219.274

Hopne, tyasine 166, 209

Hepmand, Sandra 65

Harrood, Retokah 150

Pasen Marinue 5i. 55.

Itulk Pothah 164

Heating Chris 18, 123 .

139 
Hido, Cluck 150 liestand, Jodi 150 figginbortam, Jay 136 figeinborbam. John 95. fight. Kristi i36, 24 figh. Sunh 95, 122,237 fibiah, Julia 165, 207 Elgeman, Jasos 5 IIII, Brian $15 \%$ Ial, Chrias $55,115,217$ ilil, Javias 150 ill Mishad 165 filliker, Jeremy 264 fillix, Sarah 97 fillahet, Kristen 150, 197 filly, Denuye 29, 155

fime. Joed 150, 206 fing Kolser 95, 128. finchrlman, Robert 95 finchelman, 5racy 95 Jembo, Kal 95, 236. Fod, Amanda 25.237 fobbs, Jenaifer 136, 231 Focerar, Oriatina 150. fochseserter, Preter 165 fochstatter. Peter 165 24.234

loskecberny, Lisa 150 . $03,229,242,251$ odgo, Ethan 150, 236 odiges, Andy 57 fodkenson, Ambser 150 foechoria Jeshas 15 cocke, David 74 celutralohir 200 cofl, Bethany 227.231

effinan, Brandon 165, 260 ofmans, Marthew 150 . ofmant

ofinter, Chad 150,274,

offotmer Krisy 28, 29, 95 chers, Juans 165, 224 ohman, Andrew 159 oke, Sanat 165 okef, Broavon 150, 235. olden, Mark 165, 29! giden, Michad 196,227,

\section{oldern, Phin 180}

oliday; Jans ise

ollind, Juon 190, 27

ollinger, Dwid 150 ollins, Joel 136, 206, 216 , 9.2.23

ollows, Kares 136

oimes, fernifer 136,226 . 7.235

olmea, Krota 95,216

olre. Mendy 74

oober. Auson 165

soler, Chritinas 169

oorlet, Krisin 74.98
4.265

appe, Micheie 1365

arnlateb Jowe 165,234.

ornbrook, Din 150, 189 orne, Michelle 95, 206 ons, Juitin 165, 260 pitotler, Chrisen 150 ouck, Justin 165 puck, Justin 165

pwand, Jeninifer 95

owad, Timody of

pwe, Andere 264

Dewd 164

pher, Grace 99, 236, 23

Whet. Katen 96, 237

pack Rooy 220

pak, Scod 220 .

pdvon, lerenyy 150

Feni. Magas 165,208

Eff. Debbic 150, 205, 216

fifras. Rebelah 150 .

4,236

Cycen. Justin 150

Fepen, Chris 138

When, Gits 74, 27

- When, Juritin 189

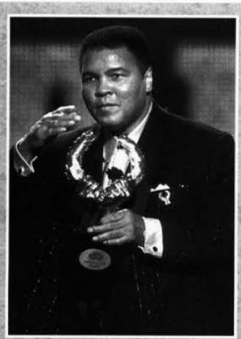

Boxity legend Muhammad Ali blows a kiss to the audience after receiving Sports Illustrated's 20th Century Sportsman of the Cencury Award on December 2. 1999, in New York City.

Hulkert Jarses 18,965 Hula, Pamila 95, 348 Hume, Allinas 138, 177 Hummiaas. Lawe Humimiaseb:
106, 206, 236

Husuberger, Joouthen 165 Huat, Jouica 165
Hust, Lrigh 74 Haw, Roper 96, 220 Hant Sas 138, 260 Henier, Alia 165 Munter. Bvian 165 Marley, Jeuring 96 Hullow, Jodi 138. 244 Hakk. Ohrisy 190 Hutecy. Heith 150.206 Huater Nials 169

Hater, Brian 39

Hutehine. Misah 136 299, 278

Hutshins, Sanuel

165. 275

Huschinene, Chria

139. 224

68

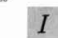

Iden, Abby 165, 209 Iapenell, Jeneifer 16 138. 196

fieland Ohat 165

275

Imin. Nathan 165 Lhell. Anber 165. $218,229,231$ Ihikive Motai 150 laskainem, Kelly 165

Laker, Weitan 13!

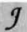

Jackun. Fenther wo

Jackion, Jad 165. 260 Jacksen, Katie 99

155, 204, 236

Jackeon, Rachel 150

Jacobowith, Jewica

$150,177,296$

Jeobs, Jack 60

Jakpmes. Foy 150

Jensen, Sieve 64

Jarih, Andoew tiat

189

Jakitas Mark 169

Jefiries, Jewerny 61.

Jasungs joy 151

227.7 Jermash. Jame $6 \mathrm{t}$

Johanaen. Graser of

Johnuen, Ben 165 , 19

Jativary 1, 2000.
Johnien, Elizibeth 96, Keller, Lairs 138, 231

203 Keller, Naulie 97

Johnow, fames $97.125 \quad$ Kerme, Heather 97,209

Jahnoun, fosic 165 Kesses Andien 97, 199

Johsion: Josh 165,190

Johason, Kaley 151, 195, Kemptos, Rath 6!

Johnesen, Kathryz 19.97

Johann. Mantics. 1 156.

$165,201,254$

Jahusion. Pumela 67,26

Jobnian. Tara 165

Jaheion, 200.236

fally Laan 165

Janch Briecy 97,120

Josen, C.Tic 158

fonse D.C 17

tonich lenvifer 259

Jonse locelyn 97,257

Josen, Melinsa 15!

Jons. Williamt 65

onker, Ktut 296

onkes. Kitiona

Jondan, Jeffrey 97

fillon, W

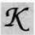

\section{Kaeriches, Mut}

Kaines 1 has

Kamiasky, Beajamin

$158,2.79$

KanArl. Kelit 191,190

Kane, B21 166 264, 265

Kane, Jon 166

Kane, Ratan 106

Kantes Surh 139

Karilerg, Paal 166, 202

Kar, Kriath 166

Keeace Kerty ?7

Kese, Dinis 97

Kecivous, Meredint 199

Ker. Datuehy 166

Koedv, Mistad 192. 226

Keenus, Derila 151

Aetenct. Ben 195. 22

Kerib. Karen 206

Ketimun. Beihny 15l, 26

Kelloter, Orit 189, 224

Kenay, Steven 151

Kenwiorthy, Lauren 135

Kentyon, Kandace 13s. 240

Kipner, Katic 151 , vis

Keri. Kriaten is!

Kern, Rashd 135, 19:

Kender, Jame 151

Resslex, Kelli 151

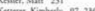

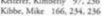

Kibelled. Nathan isi

Kick. Amande 166

Kickbuach, Kevin 166

Kicktrash, Kyle 97

Kilgane: San 166

Killer. Kriaten 200

Killisn, Kelly 134, 225

King. Antrea 97

King, flvin 67,258,

Kirg Joye 74

Kagz. Karen 97, 123,

216, 220

Kine Nicolcte 165 .

King. Toi 74

Kinney. Naomi 115

Kinnisureh. Heid

38, 201, 229, 24,

Kinsey, Heidi 97,25

Kipp, L:

Kirby, Charles 166

230

Kisby, Jonnachan 15.

Kirby, Lueen 151

225. 23 ?

Kirby, Retorect

Kirbr, Robers of

Kisctiner. Amand

106,232

Kirilund Cemers 138

Kiver, Clad 166

Kinart, Elisaherk to:

Kieix. Jonathin 212

Kiska, Katem 166

Kline, Kristren 151

Kline, Strohanis ISE

Konerer Liode ISI.

Kinas Mellaw $15 \mathrm{t}$

Knoer, Josh 166.262

Knewler Erin 233

Knudaan Karm is

Kacher, Bub 97, 198

200, 208, 233, 235

Kecher, Todd 166 .

Kacheion, Katis 134

Kedens, Kolliy

Kompen, Katir 138, 191

Kestbel, Mistael 97

Kesssiec Lyan 13

Koht Clina 64

Kok, Heidi 166

Koknay, Kanin 15t

Kolaw
294

Koncpaiek: Daniel 166

$176,151,20 \mathrm{~A}$

Kooy.Juliann 166, 201

Kopp. Timoth

Kotciy, Kristi-Lyna 216

Kormach, Bnd ISt

Kestrudk, Michaef 98

Kotawote Ki heloeno 245

Kovernan, Jand 151, 2662 264. 265

Koulowki. Bechy 144,251.

272

The infimous ball in New York's Time

Squase drops to signify the start of a new

millenium just after midnight on Sarurday,

Krick, Debbis 151,273
Koum, Andrea 98, 224

Kremdiad, Deras 138, 216

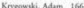

Kistia, Hall, i51, 215, 23s

Kuha. Krivin 98, 225 .

Kuscr, Kristhloyn 166.

202. 210

Kurowida. Serpharat 138

Xutuvilla. Heather 65

Kurtes, Rean 151

Kumbinikers, Foty sy

Karper Eais I64, 204

Rwait, Donid 98, 198 
Lopes, J. Michael 68 Lorines, Jarnd 167 Lougure, Michad 195 Loudemilk, Trats 151 Love, fane 151 Lovel, Amy 152,236 Low, Fdwasd 153 Lowe, Katie IGT Lowry, Cant 16 t Lowsteter, Mambew 16 Lommerter. Micharl 199 Loven, Retwece 167 Lubbes Craig 159 Lucatini, Ohris 167, 194 Luav, April $152,17 \%$ Lucas, loth 199, 201, 236 Luck. Madhad is

Lectmanas. Nicok 99, 197 Ludtua, Courtary 199, 198 Luke, Bundon 100
Luare, Jen 199, 195, 221 242

Luri, Dob :30, 24 Lurr, Jevica too, 111, 209 turn, fobert isy

Lyndon, Angie 15?

lynac, Ben 152, 210, 224

Lyom, Diana 100

Lyon, Marmall 30, 152

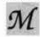

Marai, Jeray 40,200 MacDonald Emily 16? Mackin. Chris 152.207 Macthersen. Lia 257 Macpherson, Lia 19 Madien. Noelle 167 Magin Sama 167,198 Magnuwan, Jeve. 167, 206,

Maht, Keing 92, 160, 122 Makl Robret 152 Maik, Nick 152 Maiale, Kriseten 152 Mikha. Hartic 167 Major, Tim 16 ? Maller. Jetcur 190, 191 Mallnan, utia 152 Mallman, Maribeth $16 \%$ 190. 191

Malone. Kuthering

Malwin Dive 4a 139, 144

Manameiz Lani 153

Manchesky, Jwen too

Mandieser, Doasl4 139 201

Mandiga, Keinty 167, 225, 234, 239, 242

Magin Bran 274.275 Mtani, Nutas 167, 238 Mania, Rotekah 199 Manufidd Katic 199 Manifeld, Ken 152,200

Manificld, Ken t52.

Manuon, Paul 139

Masuinen. Joef 235

Manwaten, Joel 139,244

Mamialler, Rebekah 167 .

Mas, Jenias 167,203, 226

Mapes, Alliona 152,20 )

Mapen Karen 152, 195.

219,227

Mapres, Kian 100, 195, 207

Mappos, David 66

Margers, Aana 100

Marrens. Jereny 100

Mariser, Amunda 229

Mariagr, Amuinca 225

Marks. Carel too. 2?

Marks, Jestica 139

Marks, Joch 278

Marks, Ryan 15,

Marlet, Enis 167

Marlier, Karie $15 ?$

Marosa, Roscmatic 100 ;

194

Maryoudts, Jeona 36

Marn. Damid 160

Marih, Nlicia 167, 177, 200

Slank Killy 28

Sarsh, Mike 152.224

Marih. Nantwaice 167.

Starshall, Ders 167,224

Marhall. Jaen 29, 260, 261. Markell Methes 199, 17\% Markhall, Rob 16, 152,200 252

252

Mariene. Weady ise

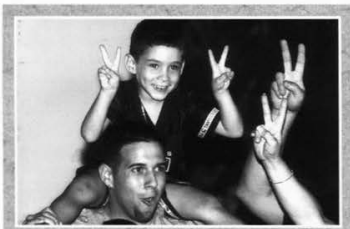

Suxcyear-old Flian Gonzaler celebrates with friends and relatives Friday, January 7, 2000, at the home of his relatives in Miami. FL after being subpeonaed to appear before a Congressional committec on February 10, 2000.

Marti. Seth in McKinney, Josh 165

Maria. Any 152.248

Marian, Marthew 139
Marin Seth 31, 139, 214

Martin. Swas 167

Mirtines, Jouepb 167

Masos, Jaha 100,192, 206.

216,224

Masoa. Tristas 167, 2!

Maw, Jeanifor 199

Mavre. Jeceny 152

Mast, Antela 108,26 ?

Vauspen, Kaic 157. 178

218,243

Mate, Mepan 167, 198 2y

Mathews. Celby 16?

Mattian Jesica 1 192 194

201, 216

Matian. Dowd 69, 124

Matheme Mart 75

Matrick, Serphen 195, 239

Matron. Joratian 167225 24a

Marmon. Laray 75

Maurbain, Jalie 231

Maurisi, Jasict 199

Muahari, Ruter 206

Mayhary. Heather 100.24 ?

Mugtury, Ruhir 152

Mower, Sarith ion, 206,297 194

Maybew, Ellie 152.230

Micilienes, Jenes 139

$\mathrm{McClain}$. Demid 167,297

MaClain. Mark 65, 226

Maclow, Mally 100,206

Miclues, Stechen 16 ?

MrCoean Brmianin too

McCoenb, Tamana 100

MdCenks. Tim 61

Micoy. Daniel 139.177

Maceacien Mall 167.200

MeCubbia. Phil 192

Mocamber, Mincelta jes

190. 191, $21 \%$

MeCene, Adam

MeDinich, Erin 139, 201

210. 238

MeDonald, Des 75,226

23 !

M. Deaneth, Arendan 152,

200

MoGher Ryas 199

MoGilliray, bentric 75 .

Mogoldrick lane 72

Mories Dovid 3k. 243

MaGaim, Amuada xt, 100.

104. 119.237

Maguim, Sarsual tiat

MoGiunsigal, Katie 16

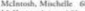

Makinna Anese 160

MaKky. Krily 210

MCKalver Heifi $101,120$.

200,21

Mikentic, Daw $19 \mathrm{y}$

Makiney, Cheik 75, 77

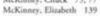

McKinean, Noet 259

Mcluin Siephanit 152

177, 195.226

Meleod, Joel 10 :

MeLeod, Jadi 152

MeManness, Jessia to

MeMurray Sturog 168

McMuray, Stepticn $24 \mathrm{t}$

McMuriy, Shavon 17

McQuinn. Emily 192. 196.

MeVey. Kric 263, 268, 263

Meach. Drew $19 \mathrm{M}$

Mead, Any 101

Mechler. Scott isz

Meisuner. Jenica 101

Meiniles, Detborsh 139, 23 ?

Melchert Amunde tot, 225

Melisah, Andien 139

Mese, Rechet 101.210.

Merot, darou 40,139, 195,

270

Meike, Jaien 169

Meriin, frod 75

Menisert. Door. 168 $21 \%$

Merert, Reth i6s.247

Servern. I I mes 189, 268 269

Merthe Jemen 139

Mesaler. Kriseih 152

Mevirc Ratorkah 267

Meree, Oend 152, 268

Morr Mtion 152,203

Meren, Hastal 169

Mevern, Marcn 139, 246

Michael, Aen 152

Midk. Brenda 101, 20 ?

Mickey Jeneph 152, 194.

Mied. Hedeca 152 .

Mikel, Trminy 192. 180

Mile. Berh 231 .

Miller. A

Miller, Brad 168,260

Millex. Cheyd is

Mellet Enily 168

Millen Erin 168, 19

Mallet Eve 168

Millex, Geac 61

Millen, Haley $13 \%$

Miller, James 168

Miller. Jesica 168

Miller. Joshus 140.

Miller, Kvite 226

Mallen. Megan 152. 238

Maller, Nicholas 56. 208

Milles, Pual 168
Malles, Restelle $>0,15$ ?

Millon, Shime 15 ?

Milier Trm 140

Mille, Hetwe 152,27

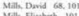

Malle Fisabers $10 \mathrm{t}$

Mill Jenviter 152 . 701.

Meribus Michuel $17 \pi, 180$

Minas, Dan 177, 179, 181.
Menect. Cyndi 69,296 , 20?

224.281

Winier, Keith 260

Minier Marthes 241

Minier, Sarn 24, 26, 28, 29

123

Minnick fall 140

Mina. Lindsy 168

Misuk, Jill 169, 240

Mitshel, Ben 168, 206, 227

Mirchell, Dianc 75 .

Mischell, Eric 152, 206

Mitchell. Micah 220

Mitchell. Paul

Mischell. Fachel 149.297

Mirchell. Todd 140

Max Jeretiy 1 ia

Merer. Jonirhun 158

Maberg. Sawaina 206

Maberg, Shanna 168, 306

Modica, Andrea 168

Mohlex, Ben 238, 239, 244

Mohler, Holly 153

Mohket, Jamic 153 .

Mohlez, Rachat 153, 207

Morsilsan, Jeanne 102, 233 Mra. Kyle 102, 260

Mudrey, Lwan 102

Mullen. Amainda 102.237

Mullea, Jortary 202

Mulvanry, for 140,235:

238. 241

Marnman, Katie 250

Mamnern, Fhilip 153 213

Mtunne, Michurl 102128

Maoin. Jeremay 168

Mondecti Murray 70.

Muedeck Jumes 102.119. 201

Murphy, Aany 103

Marphy, I. Dake 61

Murply, Sant 168, 219,246

Muraar, Darias 16s. 206

Mwangi, Roy 140, 234, 236

Mycaka. Crathia 14

Mycer. Jant 140

Mires. Jain 109

Myen. Jehn 39, 102, 275

Myen. Kim 7o

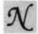




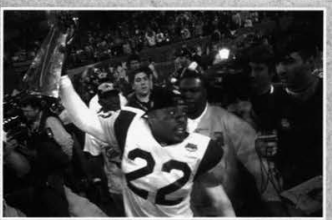

St. Louis Rams safety Billy Jenkinis holds up the Lombandi Trophy after the St, touis Rams defeated the Tennessec Titams 23-16 to win-Super Bowl XXXNV in Atlanta, GA, on January 30,2000 .

\section{Pispons, Fomalli 169}

Pierre Diniel 169

Piern, Jerumy 144, 179

$197,224,230,241$

Piesson, Jason 177,220

Pierim, Amasdo 154, 229

Piancy, Jill 154

Pienas, Robya 154

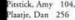

Phatic. Danid 154

Phatje. Mart 105, 123

flar. Crey 105, 215,221

Plorg, Jemnifer 141, 195
Pliment, Suran 105, 227

flumerer, Suran 105, 27

Poeiman, Amie 141

Pociaer, Mrike 15.

Jusinki. Mdlody 76

Dothinus, Lindser 28, 29

foticani, Vancas 105, 225

folland, Amy 105, 225

folland. Chriseogher 134

pollock, Surath

folluck, Saral 276

colueorfer, Rulan 76 .

fobdorfer, Redan $105,194,22$

foll $A$ mir $105,126,120$

bod, Cruatupher 105

59 . Sardh 154,199

08. 209.243

ostimait, louh 154

fotiet, Das 141

fotret, John 76

otinr, Megin 169 ,

cowell, Frick $16 \%$

fowelli, Jeft 268

wwell, Mathes 154

owell, Nicholar 141, 213

rado, Shelly 141

ralt, Dave 14

restan, Cheise 105, 220

ครเo日. PLilip 169, 19?

Eano. Andicw 165

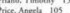

oce. Dan 144. 154, 180

(c) 159

fice, Rath 14 ?

rima, Asgets 105

rince Krinten 203

wheser Cindy 169,250

Mept, Marteni 105.216

Mnak, Eria 141

eoresther, Michud 158

towing, Kile 206

rach, Ruth 76

wachs Tim 141, 241

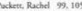

fyna, Enibeth 169

fugen Rebelat 161

wify. Viction 169.260

ummel. Kelly 164

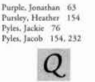

Quisa, Julic 169,227

Quias Jodi 105, 277

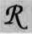

Racine. Jesie 109

Raddiffe Joth 292

Ragle, Xillam

Rainabergen, Dan 105

Rambihur, Nicia 154, 19

Raskin, Stephanie 160

Ravy, Brien 105

Raver Tamana 76 id

Ramasiach, Stacer tos

Railiff. Amanda 109

Ras. Alice 16\%. 208

Raskh. Rabys 154. 19)

Remmend Andere ios

Ryynor, Brionat 169, 19

Resd, Michad 106

Reter. Mrtanit 170. 207.

229. 239
Reckrenwald, Chris 105
197,26 ?

Recleenwald, Geodtry is
Redifingtas. Nuemi 9 ?

Redfeaine Chrisies 33,61,

105, 297, 299

Rediager. Kellie 170

Redingron. Nueni 106

Redmeed, Dizmond 154

Reed, Aay 170, 197, 221,

Red, Caiey 170

Rermases. Alivon 154, 243 ,

244. 248

Kege Iff 200

Eotnca, Eache 170.206

Reid, Mas 120

Reily, Goes 21, 170, 239

Revienbigler, Dovid 106 .

235

Rria, Kelly $106,203,229$

254, 255, 276

Rorsh. Dorid 170, 225, 239

Rrninger. Angie 170,2055

Rano, Amy 141.291, 236

Rimos. Joseph 170, $21 \%$

Rima, Rached 106

Eafert Meify 14t, 202

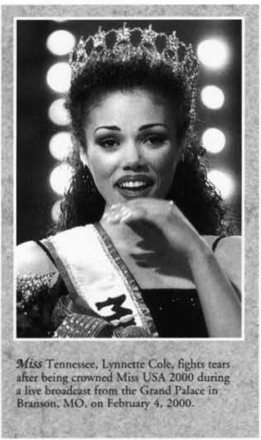

Reyen, Melisa 141, 226.

Rerselde, Clif 141, 198. 278, 279

Rayeolis, Rebchah 154,

ni, 23

Rharm. Darid 154

Rhodea, Zach 141,204,216

Rice, Clinmen

Rictudions, Gregory 170

Recturdos Machar 100

Roi

Rol Doved Den to

Redard. Dunald 69

Rickes, Dun 141.275

Rigen Jack 69

Rot Jorke 67

Ripat Charias 16!

Rahteill Ohris 209. 224.215

Riuhe

Ruine tymdel!

Rerigers Sarah 106, 195

Rirrrt. Jumie 106,204

Rivers, Hannals 106

Rive, Hannah 230

Riva, Sxm 220

Riseridas 106

Raach, Raty 170, 210

Robers, Genere: 22. 25 .

106, 202, 216

Robcrs, lod 238

Robersa, fulia 12,4, 201 iss

Robers, Kiators 170, 198 .

Bif. 236

Roberis, Kristin 170

bobrts, Lindser 17

Robey. Beverly

Rohey. Dowid 68, 181, 233

Rohin. Melisa 141

Robinine, Devin 170, 177

Robinsen, Jaspine 105, 225

Robinios, Jasand 141, 176,

Ig)

Rothiniog, Kates 154, 208

Eobinon, Mindy 106, 11 ?

Rock. Jabdle 14i

Rixike, Dancile 231

Rodriguer, Andrew 212

Roc, Brian 170, 295

Rove Feberts 14 !

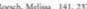

Reclert. Rinan 106, 231

Rogren, Danirlle 154, 199

Ropen, Lymn 61

Rowm Bob 8,9, 25, 63, 145

Rohin, Lren 8,9.77

Roman, Jowifor 170,26

Rooctkomski. Nouh $3 y$.

225

Ropp. Tin 14

Rone joel 196

Reen, Kate 170

198 . 154

196

227

Reas, Saral 154,

207 , Sterii 141

Rows
207

Rosscau, Angel

$70,189,206,242$

Rossesti, tirian 106

Ronsouti. Kinster

Rost Alyse 170

208, 216 .

208,216
Rost.
216

Roat,

239

Roth Gres 1

191, 208,239

Roih. Hearber 170

198. 201,236

Roman, David 63

Romart Doug 212

Roemun. Dougla

Rosph, Joknny 239

Resackes. Antols

231

Rown. Tabich 141 .

204

Rowland, foch 170 .

206
Rebere. Manatha 106.

304. 217,229

Reby, Cal 63

Bulty, Ellen 170, 192

Rudal. William 61

Rudicil, Jamar 17

Ruffin. Caner $107,241,266$

260

Rublenan Michelle 154.

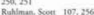

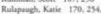

255. 276

Rumpbol, Ainher 141

Ruens, Mogas 107,226

Reshirg Lesa 170

Rusedl, Kace 170, 194

Russtil, Mick 170

Ruth. Krnt 170

Ryan, Ruchel 197, 128, 201.

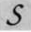

Sagk. Alan 14

Sagr. Amanda 107,225

Sallests. Courmey ipo
Schoull. Cournenay 77
Schoonaver, Brdky 170, 284 Schrismer, Kate 155, 190

200

Sathork tias 109

Schrocter, Lis 170, 299

Schruseler, Star 171. 275

Sthrye. Pattic 299

Sdunt. Rachel 171.20\%

217.23.

Schular, Dean 107

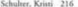

Shamacher If 107,246

Sotuemacher, Lia 171

Schusaches, Rober 68

Shuser, Kriati 155, 194

the

Schnaru. Conaic Ig)

Scon, Bvert 16?

Scoth, Cun 142

Scon. Dawn 77,264

Sent, Nicele 24

Sechriat, Beat iv

Solot, David 155

Cocom, Janeifor 162

Seriey, Angele 442, 259, 267
Shiner, gestette - 23, 142, 367

rubsele. lon 210

Shathok, France 155, 242

Shestoule, fuestan 77

Shrubogk Jucit 221

Shemen Slathes 171,297

Shuack, Jos $155,217,21$

sicand, Christian tos

Siddall, Allivan ith

Sidet, Rachel 108

Sides, Nilki 202

Gemer, Jane 171, 204

Stivak, Jahn 65

Simonk, Seoet 181, 214, 24:

Simpson, Lin 155

Sima, Kerin 71. 79

265

265

Sollinge Sanh 155

Skuedal, Berojumin 195

Sinatagh, Mrlanie 155.236

Smith, Andy 171.194

Senith Bearanin 7

Sonith. Bill 61

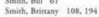


Spaulling, Nick 261 Scap. Anas $109,121,125$ Spencer, Ourity 171 Sperecer, Edwatd 69 Spiak, Cathetioc 171,29! Spizat, Brad 171 142.210.

Springirth, Nete 155. 200 237, 265, 268

Sprouli, Heidi 155

Spenill, Elirabetk 155

Stackhouse, Lin 109

scaffurd, Abby 171,272

Sufford, Andire ith

Stahley. Joah 142

Sandiuh. Aaty it?

Stakkicrice, Jeandót 109

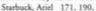

Seatk, Amy

Stark. Heather 162. 193

Swark, Tammy 109,127

Sualles, Aatos 155.275

Stats. Tiffani 171 .

Stauffe, Mdive 17

Svet, Suana 10 ?

Steck. Jonsifta $29,155,203$

Soccewik Elinbeth 155

Secerle, Edde 109

Secinueas. Kathy 155, 18?

Secingass

Stcirman, Karz 77

Sienget. Amber 155.196

Stephan, Cametas 191

Stepkens, Nathan 109
Stephens, Shown 28, 20,

142, 230, 238, 244

Stepberiv, Wea 171, 278

Stephens, William 17

Stern, Ryas 142, 208

Sterens. Albert 61

Sterese, Aminda 171,25

Stevens. Jenia 17

Surven, Sech 172, 23

Stevenasas, Craig 22

Stevensas. Heidi iog

Simenena, Jessica 216

Serwar, Ball 123, 295, 240

Somara, Edwat 172

Stewert, Rebelak 173

Seevart, William 109

\section{Sengims int.}

Stickelmaicr. Dowal 172

Saitacl. lames 142

Sockoon, Risha 142, 19t.

218,297

Sickes, Chineopher 172.

Sooltenberg, Cirls 150

5roov, jear 172

Sroner, Samine 172 .

Stoufer, Tims 239

Siowern Any 109

Stomern, Besky 142.198

Sinsitu, Chris 142, 195

Sirsler, Maitbers 224

Strang, Amy 142.209

Strang Mrurces $10 \%$

Strasler, laraca 172

Surves, Reit $20-4$

Siruch, Naha 172

Siricker, Mark 17

Simeng. Daniel 109, 117

Sitang. Dinicl 109.117

Strong Kendra 109

Sirong Seepbanie 186, 210

Struz, Diwn 143, 297

Strychalik, f II 143

Strychatshi, Joisph 156

Surktt, Jenay 45, 110, 218

Scucker, Brandea 172

Srucen, Party of

Stummun. Ben 110,198

Sudlow, Dan 156.206

Sullivat, Caig 156, 275

Solivan, Deenit 65

Sumimen, Becly 110,207

220, 272,273

Summen. Durrk: 143

Suppler, Jenice 77 .

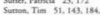

237.238, 299

Svendeen, Eric 110

Swaler, Sandra 110

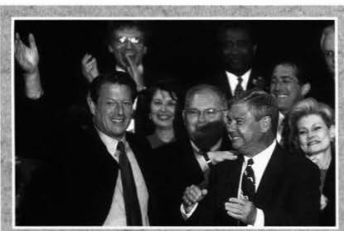

Democratic piesidential candidate ând Vice President, Al Gore. left, waves with Senatoe Bob Graham, D-FL, during a campaign rally, at Leon High School in Tallahasece, FL, March 14,2000.

Gore swept the Southern primaties that night.

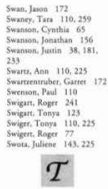

Tais, Luerie 156, 232 Tait III, Doupdas 110 Toldo, Fise 156,207 Talledery foy 110,129 Tallakaca, Heither 149

Tallaria. Tries $10 \mathrm{i}, 110$

Tallaria Theres 220

Tapp. Tiahe 149.238

Tats, Bethany 156, 194 .

210. 22 .

Tats, Finip 143.2\%

Truin, Scoet 110

Tantot Aminde 148, 144.

3os, 216

Treylet, Eleanet 267

Twhot, Jeel 143,224, 23

Tolat Stacy 110

Twolos. Sarven 207

Tehan. Dwid 177. 192

Tencia Jeremy 156

Teridiven, Suian 71

Terlouw. Karen 17

Terry. Jesica 156

Tetrick, Jennifer 172.248

Truaink, Rebelat 156

Tharngan. Rebeca 116, 209

Tharp. Michan 14, 143,24

Thayer, Kastina 110

Thippen, David 110
Thirey, Mish 38, 143, 200

Thirey Miah $38,143.200$
Thernas Jack 91.172

Themas, Merica 172, 195

2i1, 242

Thomas, Nikki $22,24,25$.

99, 110, 125, 225, 236

Themas. Puul 191

Themas, Sarah 110

Thampian. Becky 198, 20s

Thoespent. Bes 78. 116 .

194. 292

Thempeon, Brias i1!

Thempeon. Jedy 111.257

Thempsao, Petricis 111.

117

Thempsin. Rachel 201, 231

Thempeon, Rebekah it

Thempsia, Sirven 77

Thomywan, Thamei 68

Thenpwern, Rastad 156

Theraleseg Marifes 172

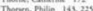

Tiderll, Sterten 172,237

Tide-

Talles. Luca 17

Tilen, Wen yo, iss
Timm. Eriks 172, 190

Tipren, Alicia 1111
Tipren. Kimberty 231

Tomkinseb, Joel 172,207

Tamkinaen, Sara 143,207

231

Toms, Ruseli 149.235.271

Toses, Rass 240

Tong. Irneifer 143

Topp. Elazabet 172

Towniend. Timathy in

Toyer, Lee Anee 111.20

Trages, Chrasibe 208

Trago, Chtiitine 196/216

Tramp-Josh 226

Treadwel., Msik 111, 195

Trimble Jenes 111. 198

Treske, Erie 111,231.243

Tremp Jochua 163

Treut, Julie 111, 196, 227

Tregcr, Jraifcr 143

Trover Jenay 232

Truver. Mary 70

Tiermatagah, Sarnh 172,272.

273

Tubbs. fusein 41, 196

Truchalike, Nick 143, 235

Tocken, Ambet 143, 199

Tucker, Mart 172, 23

Trinain, Frin 11.6. 209

Tukin, Rachd 297, 239

Tumer, Jomathan 112

Turnick, Melina 156, 26

Trice, Amy $112.1 \%$

Twige Eather 112

Tynan, Nack 155. 212

Truon. Cartie 112, 195

Tywon, Heather 112 198

Truon, Kelly

\section{u}

Uimi, Elinatherh 156, 190

Ulich, Lindssy 156

Uneinn, Kriatic 172

Underkofles, Mark 156, 197

Unilank, froan 172

Upham Nathan 756

Urbers, Jeni 143

Lrbauik, Rran 112

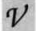

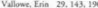

Vea Der Aa. Scetr 156, 275

Van Der Aa Steqhen 172

Vin Gorp. Heather 112,12

Van Hant, Tiftany is

Yan Heukrion, Andicy

Vis Heonct, Kaisa 112

Yen Lraten, Mark 156

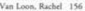

Yan Treos, Mtistact 172

Vin Turl Segheric inz.

bis

Yal Wingenden, Jelene 172
Tore, Jasen 111,214

Viut, Naom 172, 207

Vhug, Beniamin 156,243

Vogel Rran 163

Vogr, flomec 156, 196

Voigt. Jared 112, 194

Volpe Lawa $136+92$

Volece, Rachd 112.12

Van Det Metder. Jestic

172. 198

Vtamas: Benjimin 143

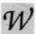

Wuadcalurg, Ty 112.210

Wabcks, Tritha 156, I05.

206

Wade, Michad 112.234.

238 Wudlingen, Mrlanie 112

Gudlingen. Mrlatie 112

Wageonet, Brimany 17

Wugnm, Kim $10 \%$

Wagrer, Jessia 112,224

Wignet, Kent 156

Wagree, Kimberly 156

Wagnes, Lindar 104. 112.

Wraner byncute 156,236

$W_{\text {agner. Puth }} 156 \mathrm{6} 19 \mathrm{1}$.

206,218

Wakefield Chester 279

Whitefield Sieve 169.256:

273

Wilberg, Karima 192

Walde, Japes is

Wilduck Kenarth 143, 198

215

Waldion, Kariasa 173, 251

Wilkrs, Dich 78

Wallors, Dusian 22, 29?

Walken fennifer 157,251 .

276

Wilken, Michefle 179

Wivere Renold ?1

Wulker, Soent 40, 92, 112 .

$216,220,244$

Walkes, Trisis 78

Wrikes, Willat 143

Willace, Jennifur 112.241

Whatlars, Marh 157

Wall

Walline Justia 175

Waluen, Best 276

Walten, Sarah 173

Walcos, Heatber its

Waln, Enndon 78

Wambild. Dawn 231

Wambold Jotn 78

Wander, Ktite 200

Wander. Melius 11

Ware Nuthan 210

Wavinuer, Pere 84, 104, 113

234

Wamet, Sharen 78

Werrer, Suaur 7 ?

Watriks, Adam 113, 128:

201.21

Wams. Alivon 143. 26?

Warten. Dwid 61

Wartiner, Auton 113,227 .

244 


\section{$-\mathrm{C}^{-} \mathrm{H}-\mathrm{U}-\mathrm{R}-\mathrm{C}-\mathrm{H}-$ \\ patrons}

Betfiany Bible Fellowship Church

Hatfield, PA

Bloom Free Will Baptist

South Webster, $\mathrm{OH}$

Centerville

Community Church

Centerville, $\mathrm{OH}$

Charlotte Baptist Church

Charlotte, ME

Emmanuel Baptist Cfiurch Toledo, $\mathrm{OH}$

First Baptist Cfurch

Merton, WI
Grace Fellowsfip

York, PA

Grandville Baptist Church

Grandville, MI

Heritage Baptist Church

Clarks Summit, PA

Mt. Pleasant

Church and Ministries

Baltimore, MD

Northfield Baptist Church

Northfield, $\mathrm{OH}$

Rocky Point Chapel

Springfield, $\mathrm{OH}$
Shortsville Baptist Church

Middlebury Center, PA

Soutfigate Baptist Church Springfield, $\mathrm{OH}$

Summerdean Church of the Brethren

Roanoke, VA

\section{Temple Baptist Church}

Portsmouth, $\mathrm{OH}$

Trinity Bible Church

Rock Hill, SC

Wakefield Valley Bible

Church New Windsor, MD
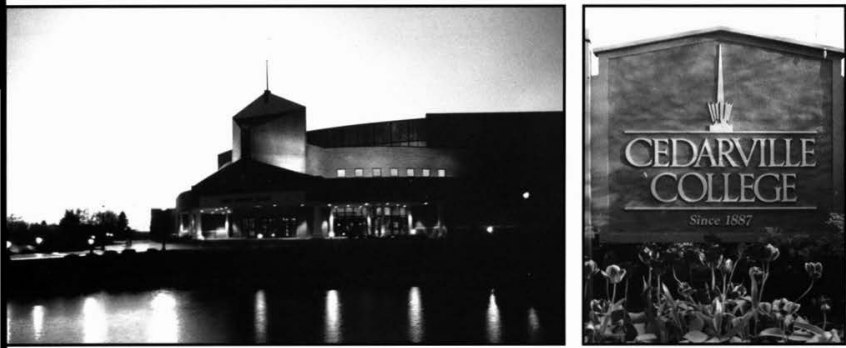


\section{$-\mathrm{P}-A-\mathrm{R}-\mathrm{E}-\mathrm{N}-\mathrm{T}-$ patrons}

Daniel Acton

Battle Ground, IN

In Memory of Jacqueline A. Acton

David and Lynn Allen

Jamestown, NY

Mr. ef Mrs. Gene Armour

Rochester, NY

Mr. and Mrs. Terry Barrons Caro, MI

David Beres

Canton, $\mathrm{OH}$

Terry and Patsy Blosser

Dayton, $\mathrm{OH}$

Mr. and $\mathcal{M}$ rs. Donald B. Bodder, Ir.

Pittsburgh, PA

gofin and Anastacia Brazalovich Saginaw, MI

AJ and Joan Brown

Tecumseh, MI

In Honor of Susan

Janenne Burgett

Fredericktown, $\mathrm{OH}$

In Honor of Tiffany $\mathcal{B}$. Loose

$\sigma$ Brooke E. Burgett

Mike of Nancy Byers

Cedarburg, W/

Ken and Brenda Caldwell

Panama City, FL

In Honor of Leah Caldwell

Robert and Jessie Carr

Newtown, PA
Mark and Donna Castelleani

Sarasota, FL

Mr. and Mrs. Gerald P. Cicchini Cinnaminson, NJ

Given in the name of Timothy Cicchini

Mr. and Mrs. Jofin Congdon

Randolph, NY

In Honor of Lucinda Congdon

Iames and Bonnie Copella

Holland, NY

Pfilip and Debbie Cormany

Rockville, MD

In Honor of daily chiapel worsfip

of the risen Lord Jesus Cfirist

Mr. and Mrs. Lawrence Cox Fairfax, VA

Steve and Lisa Cummins Arlington, TX

In Memory of Elizabeth Elmore

Frank and Faye Davenport

Essexville, MI

Daryle and Brenda Doden

Auburn, IN

In Honor of geff Cunningfiam of fis crew

Willian and Karen Dunn

West Chester, $\mathrm{OH}$

In Honor of Sarah and Amanda Dunn

Jofin and Marsha Eads

Jackson, Ml

Ron and Susan Edwards

Gahanna, $\mathrm{OH}$

In Honor of Amy Leigh Edwards
Dr. Michael Fourman, $\mathcal{D D S}$

Greenville, $\mathrm{OH}$

Edda Frank

North Royalton, $\mathrm{OH}$

In Memory of James A. Frank.

Pastor and Mrs. Webster Frowner

Kansas City, MO

Ken and Denene Furst

Appleton, W1

In Honor of Caren Furst

Steve and Christa Gruber

Greenville, $\mathrm{OH}$

Sfiarman and Aaron Hedges

Massillon, $\mathrm{OH}$

In Honor of Andrew Hedges

Rev. and Mrs. Paul Hills

Norwich, NY

Given in the name of Myron or Alura Hills

David and Gail Hjembo

New Windsor, MD

Charles and Judith Hobbs

Bosie, ID

In Honor of Bruce and Myrllin Cook

Jerry and Andrea Hodge

Franklin, $\mathrm{OH}$

Mr. and Mrs. Steven Howard

Springfield, IL

George $\mathcal{E}$. Huff, Ir.

Mansfield, $\mathrm{OH}$

Jack and $\mathcal{L}$ ancy Jacobs

North Olmsted, $\mathrm{OH}$ 
Teresa Jackson

Rolla, MO

In Memory of fier fusband, Iim gackson, USMC Ret, and the 8 Cedarville

College students who ministered to fier and fier family after fiis passing

Frank and Barbara Jenista Vienna, VA

Timothy and Linda Kellefier

St. Charles, IL

In Memory of Joseph Kellefier

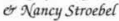

Ed and Ruth Kipp Beloit, WI

In Honor of Erika Xipp of Yolin Bourdeaux

gerry Kurzen

Dalton, $\mathrm{OH}$

Jofin and Estfier Lacy

Farmington Hills, MI

Mr. and Mrs. Jänes Low

Clinton, MD

Michael and Bonnie Luck

Ft. Washington, MD

Clarence and Edne Mast

Tunkhannock, PA

Elizabeth McKinney

Louisville, KY

Tom or Pat Miller

Monroeville, $\mathrm{OH}$

Mr. and Mrs. Micfiael T. Mills

Dayton, $\mathrm{OH}$

Fred and JoDee Nelson

Vicksburg, MI

William and Michiyo $\mathcal{N}$ (oble

Tunkhannock, PA

Given in the names of

Charles of Edith Noble
Bruce Offinger

Columbus, $\mathrm{OH}$

In Honor of Justin Offinger

Frank and goyce Pereira

Hagerstown, MD

In Honor of Janene Pereira

Mr. and Mrs. Ronald Pierre

Oberlin, $\mathrm{OH}$

George and Sandy Pilgrim

Frodonia, NY

In Honor of Amanda

Harold and Patti Ploeg

Grand Rapids, MI

Dave and Jan Recktenwald

White Lake, MI

In Honor of Christopher Recktenwald

Jeanine Reilly

Glen Ellyn, IL.

Given in the name of Mimi Jordie

Jofin L. Robinson

Verona, PA

Jerry and Sue Smith

Elmer, NJ

In Honor of Jaclyn Marie Smith

Ronald and Susan Smith

Johnstown, $\mathrm{OH}$

Keitf and ㄱancy Svendsen

Algonquin, IL

In Honor of Eric Svendsen
Ron and Pam Tillet

Rensselaer, IN

In Honor of Wes Tillet

Deboraf Tsermengas

Ecorse, MI

In Memory of Thomas 'Worley

Dennis and Louise Fwigg

Cincinnati, $\mathrm{OH}$

Ed and Katfii VanderBush

Downingron, PA

In Honor of Jennifer Marie VanderBush of Edward $\mathcal{F}$. VanderBush III

Jofin and Adele Weeks

Racine, WI

James and Lynda Wishart

Canton, MI

Clint and Catfry Yale

Bellingham, WA

In Memory of Les Ryberg

Mr. \& Mrs. Cliff Winburn

Tulsa, OK

In Honor of Katfryn \& Katfy Winburn

\section{Stephen Ziel}

Comstock Park, MI
The 2000 Miracle

thanks all the parents and

churches who contributed

to the cost of this

yearbook. We greatly

appreciate your support. 


\section{PRODUCES A YEARBOOK}

After countless hours of production, the 2000 Miracle Staff, comprised of over thirty-five members, rested with the satisfaction of having completed all of their responsibilities. The staff was led by four editors, Emily Bohl, Ben

Gayer, Rob Moll, and Catherine Wayne. In addition, Krista Warder worked during fall and spring quarter as an assistant editor, contributing signifcantly to layout, page design, and photo manipulation. Jessica Haynes served as the administrative assistant throughout most of the year. The staff members attended weekly meetings and completed their work independently. Mrs. Cyndi Messer served as the yearbook advisor. In addition, Mr. Terry Chamberlain served as the layout advisor:
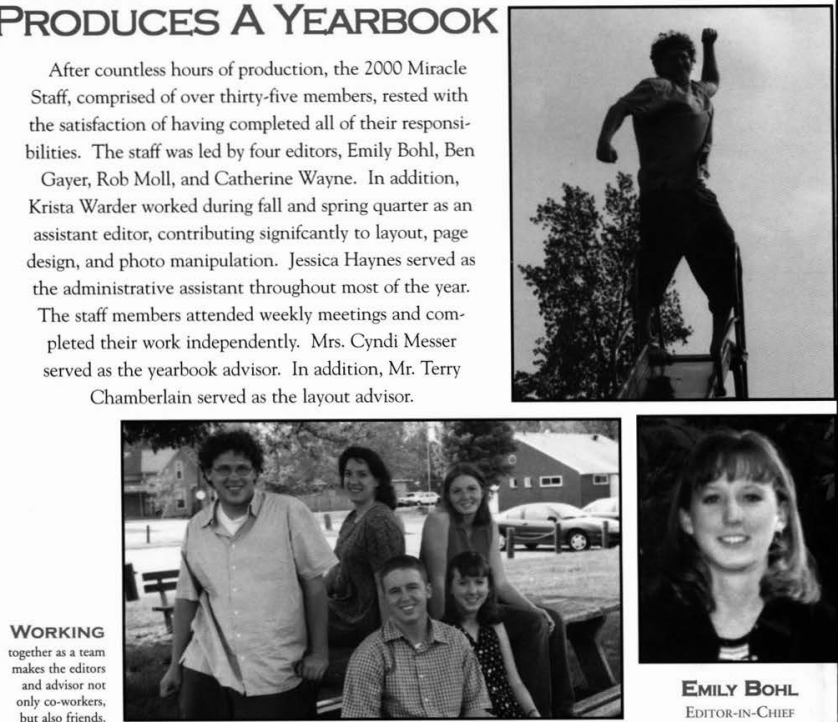

EMILY BOHL

EDTTOR-IN-CHIEF

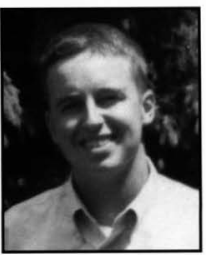

Ben Gayer

LAYOUT EDTTOR

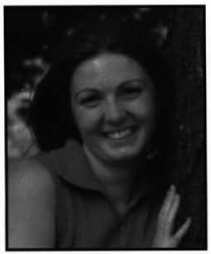

Catherine Wayne

Photography EDTTOR

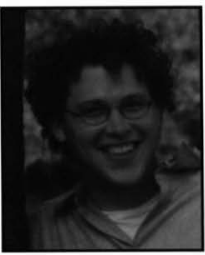

Rов MOLL

COPY EDTTOR

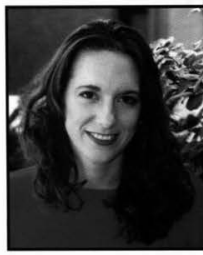

KRISTA WARDER

Assistant EDTTOR 


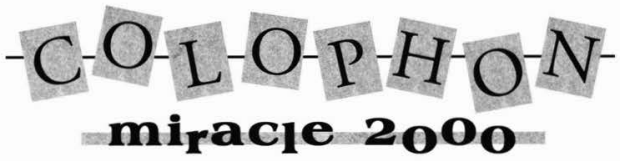

\section{publishers}

The 2000 Miracle was published by Jostens, Inc. It was printed at their plant located in State College, PA. Our Jostens representative was Tillie Billheimer. Our plant representatives was Tom Yontosh.

\section{Cover}

The 2000 Miracle cover was an Craftline cover, printed on Matte Black 480. It was rounded, sewed, and bound. The front and spine lettering was embossed and screened with Pantone color $402 \mathrm{C}$. The tip-on was printed in fourcolor process ink and applied by Jostens. The endsheets were printed on Sterling Silver 420 with black text on the front endsheet.

\section{paper stock}

The 2000 Miracle's 304 pages were printed on Glossy 80 \# paper.

\section{Color}

The 2000 Miracle contained eighty-eight pages of fourcolor process ink and photos. These pages were located in the opening, activities, senior, and closing sections. The senior section contained a textured background applied with Pantone color $402 \mathrm{C}$.

\section{design}

The 2000 Miracle cover was designed by Emily Bohl and Krista Warder. The opening, dedication, dividers, and closing were designed by Krista Warder. Section layouts were designed by Emily Bohl, Ben Gayer, and Krista Warder.

\section{theme}

The 2000 Miracle theme, LuX SUM, Latin for I AM THE LiGHT, was selected by the editors of the yearbook. It was chosen as a reminder that despite the unknowns of a new millenium and era, the truth that God is the light of the world remains unchangeless. In addition, the call and need for Christians to live their lives as a reflection of God's light in the darkness of this world is still a reality. In the year 2000 and beyond, it was the editors' hope and prayer that the Cedarville Collegestudents, faculty, staff, family, and friends would remember this truth and live their lives in the Light.

\section{production}

The 2000 Miracle was produced on IBM compatible computers. Layouts were produced using Adobe PageMaker 6.5. Photos were scanned using HP Precision Scan. Photos were cropped, resized, and manipulated using Adobe Photoshop 5.5. Text effects were produced using Adobe Photoshop as well. Copy was typed into Microsoft Word and then imported into PageMaker.

\section{typography}

The 2000 Miracle contained a variety of fonts throughout the book. The cover, dividers, folios, and captions were printed in Adobe Garamond. Stories were printed in Goudy 11 point. Headline and highlight fonts varied by section: opening-ITC Zapf Chancery, activities-ITC Zapf Chancery, chapel-Garamond Light Condensed, academics-Parisian, seniors-ITC Zapf Chancery and Americana XBd BT, underclassmen-Parisian, arts-Copperplate Gothic Light, ministries-Parisian, organizations-Copperplate Gothic Light, athletics-Arial, closing-ITC Zapf Chancery and Americana XBd BT.

\section{photograhy}

The 2000 Miracle photos were taken primarily by staff members. Cover, opening, and closing photos were taken by Kim Leverson and Krista Warder. Ministry and organization group photos were taken by Evan Warble and Rich Ormanowski of DaVor Photography. Graduation photos were taken by Evan Warble of DaVor Photography. Sports team photos, play photos, and concert photos were taken by Scott Huck, Staff Photographer for Cedarville College Public Relations. In addition, additional photos were taken by Scott Huck as credited on the pages where this occurs.

\section{inquiries}

The 2000 Miracle may be contacted for additonal information about its production by writing the staff at Miracle, Cedarville College, 251 North Main Street, Cedarville, OH 45314. They may also be reached through e-mail at miracle@cedarville.edu. 


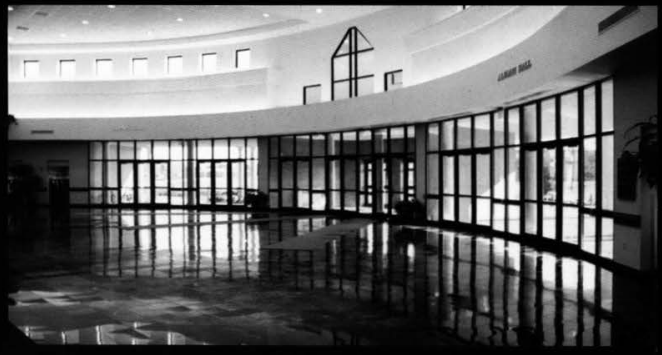

But you are a chosen generation, a royal priesthood, a holy nation, His own special people, that you may proclaim the praises of Him who called you out of darkness into His marvelous light. ...

I Peter 2:9
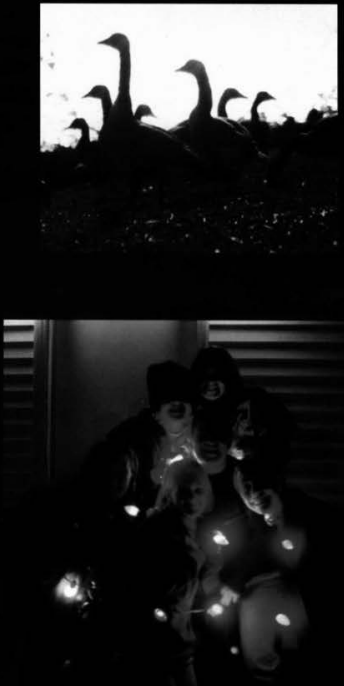

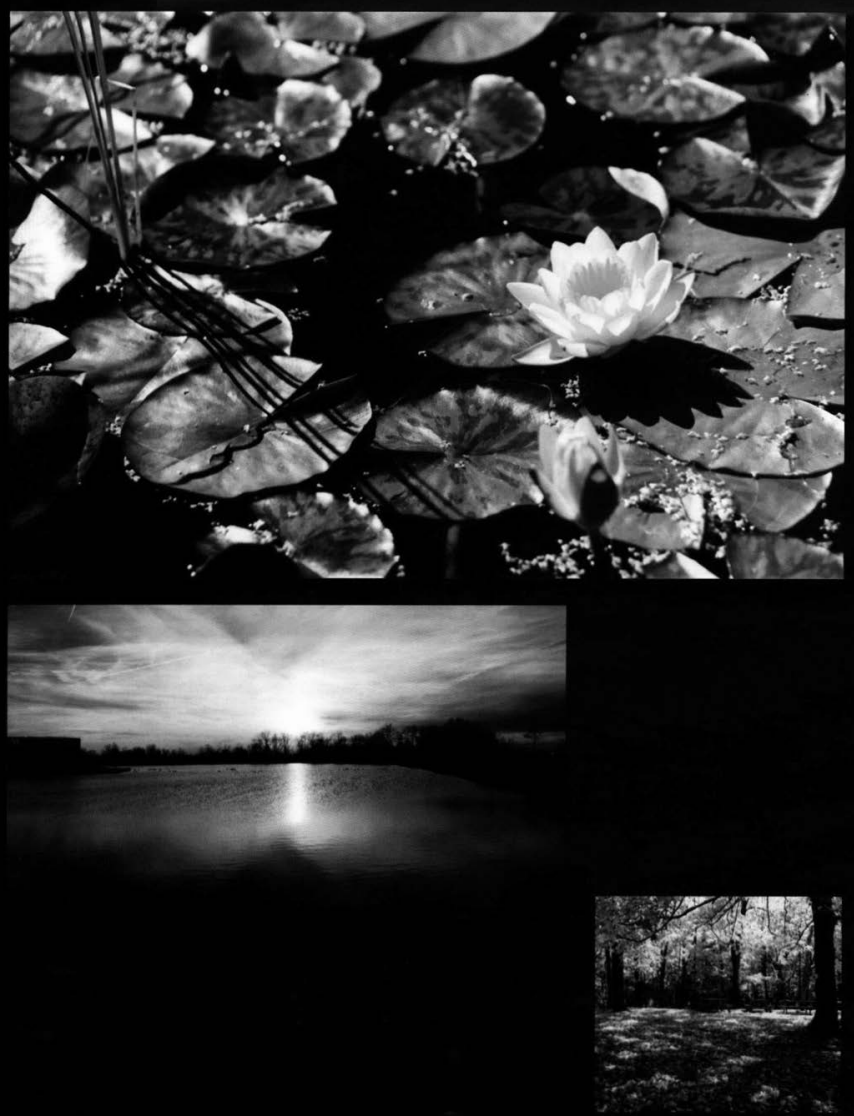

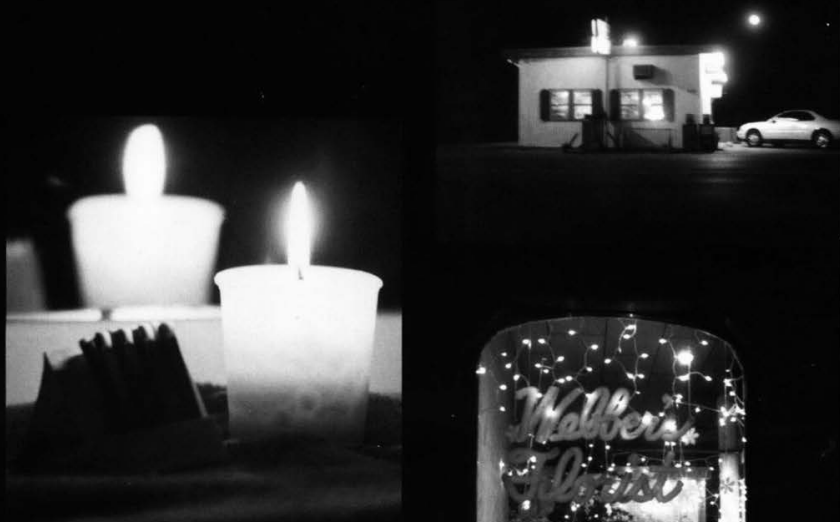

For God, who said,

"Let light shine out of darkness," made his light shine in our hearts to give us the light of the knowledge of the glory of God in the face of Christ.

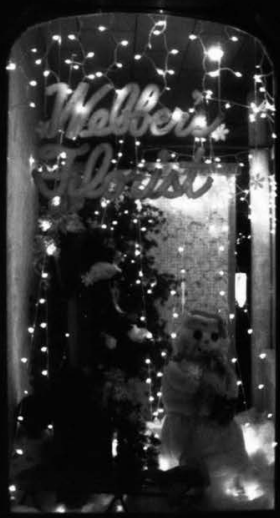

II Corinthians 4:6 


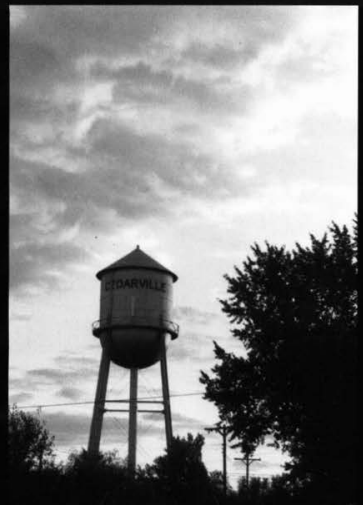

ite
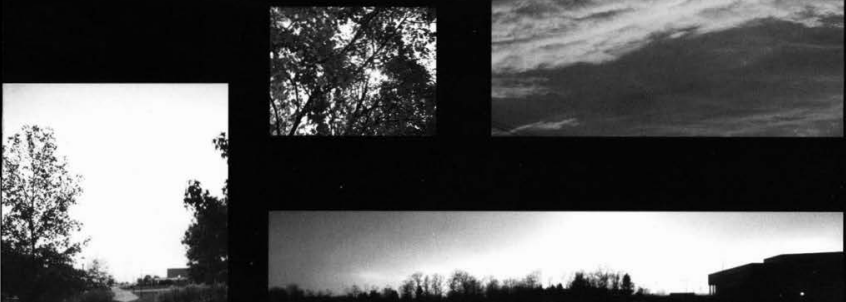

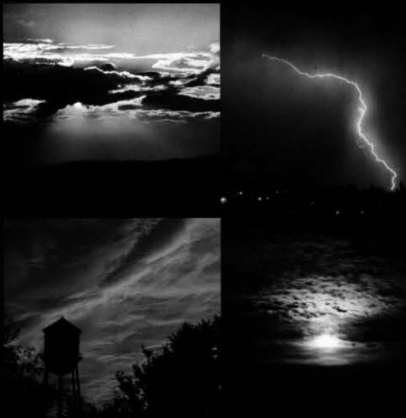

I AM THE LIGHT 



a.

4

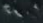

tot

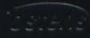

